Supporting Information

\title{
Discovery of nucleic acid binding molecules from combinatorial biohybrid nucleobase peptide libraries
}

Sebastian Pomplun, ${ }^{1}$ Zachary P. Gates,${ }^{1,+}$ Genwei Zhang, ${ }^{1}$ Anthony J. Quartararo, ${ }^{1}$ Bradley L. Pentelute ${ }^{1-4^{*}}$

1 Massachusetts Institute of Technology, Department of Chemistry, 77 Massachusetts Avenue, Cambridge, MA 02139, USA

${ }^{2}$ The Koch Institute for Integrative Cancer Research, Massachusetts Institute of Technology, 500 Main Street, Cambridge, MA 02142, USA

${ }^{3}$ Center for Environmental Health Sciences, Massachusetts Institute of Technology, 77 Massachusetts Avenue, Cambridge, MA 02139, USA

${ }^{4}$ Broad Institute of MIT and Harvard, 415 Main Street, Cambridge, MA 02142, USA

† Present address: Agency for Science, Technology and Research (A*STAR), p53Lab, 8A Biomedical Grove, Singapore 138648, Singapore

*Correspondence to: blp@mit.edu 


\section{Contents}

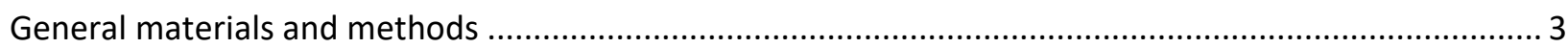

Preparative organic synthesis of Fmoc protected nucleobase amino acid monomers............................. 4

Schemes

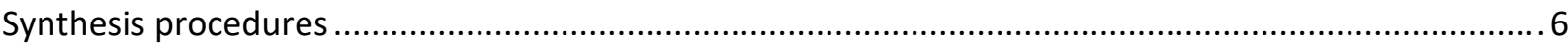

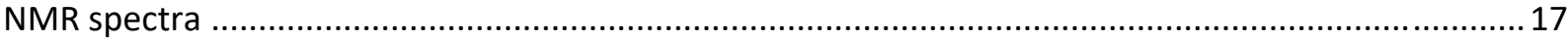

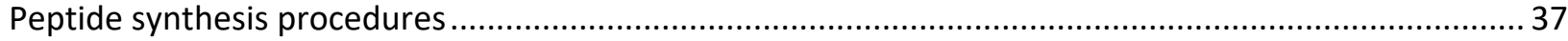

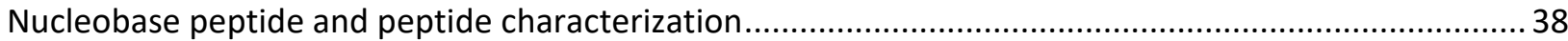

Synthesis and de novo sequencing of three nucleobase peptides test libraries ....................................52

Synthesis and characterization of a $10^{8}$-member nucleobase peptide library........................................67

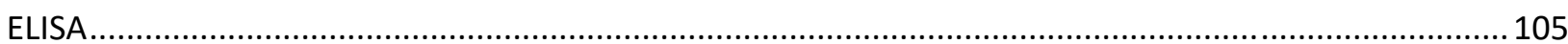

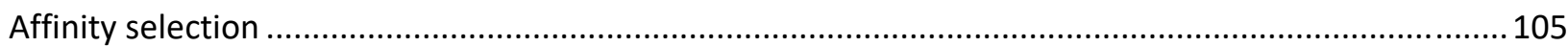

General procedure for biolayer interferometry (BLI) .................................................................. 106 
General materials and methods

Chemicals: Unless otherwise noted, all chemicals were obtained from commercial sources and used as received without further purification. Anhydrous tetrahydrofuran (THF) and $N, N$-diisopropylethylamine (DIEA) were obtained from a Seca Solvent Purification System by Pure Process Technology.

LC-MS analysis: LC-MS chromatograms and associated mass spectra were acquired using an Agilent 6550 ESI-QToF mass spectrometer or an Agilent 6520 ESI-QToF mass spectrometer. Mobile phases used for LCMS analysis were solvent A ( $0.1 \%$ formic acid in water) and solvent B ( $0.1 \%$ formic acid in acetonitrile). The following LC-MS methods were used:

Method A: On $6550 \mathrm{MS} ; \mathrm{C}_{4}$ Phenomenex Jupiter column $(1 \times 150 \mathrm{~mm}, 5 \mu \mathrm{m})$; LC conditions: $1 \% \mathrm{~B}$ from 0-2 minutes, linear ramp from $5 \%$ to $61 \%$ B from 2-12 minutes, $0.1 \mathrm{~mL} / \mathrm{min}$ flow rate.

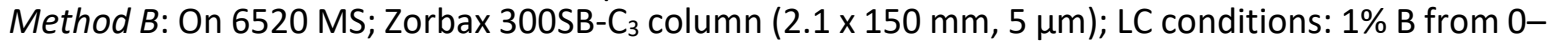
$2 \mathrm{~min}$, linear ramp from $5 \%$ to $61 \% \mathrm{~B}$ from $2-12 \mathrm{~min}$, linear ramp from $61 \%$ to $90 \% 11-12$ $\mathrm{min}, 0.8 \mathrm{~mL} / \mathrm{min}$ flow rate.

NMR analysis: NMR spectra were acquired on 400 or $500 \mathrm{MHz}$ Bruker spectrometers at ambient temperature. Samples were prepared in chloroform- $d$ (DMSO- $d_{6}$ or $\left.\mathrm{CDCl}_{3}\right) .{ }^{1} \mathrm{H}$ NMR data are reported as chemical shifts with multiplicity, coupling constants $(\mathrm{J})$ in $\mathrm{Hz}$, and integrations. Proton chemical shifts are reported in ppm $(\delta)$ relative to tetramethylsilane $(\delta 0.00 \mathrm{ppm})$ and referenced to residual solvent $\left(\mathrm{CHCl}_{3}\right.$, $\delta 7.26 \mathrm{ppm}$ or DMSO- $d_{6}, \delta 2.50 \mathrm{ppm}$ (quintet, $\left.\mathrm{J}_{\mathrm{HD}}=1.9 \mathrm{~Hz}\right)$ ). ${ }^{1}$ Multiplicity is reported as follows: singlet (s), broad singlet (bs), doublet (d), doublet of doublets (dd), triplet $(\mathrm{t})$, quartet (q), multiplet $(\mathrm{m})$, and overlapping multiplets (comp). ${ }^{13} \mathrm{C}$ NMR spectra were recorded on $500\left({ }^{13} \mathrm{C} 126\right) \mathrm{MHz}$ Bruker spectrometers with proton decoupling at ambient temperature, unless otherwise noted. Carbon chemical shifts are reported in ppm $(\delta)$ and referenced to solvent $\left(\mathrm{CDCl}_{3}, \delta 77.16 \mathrm{ppm}\right){ }^{1}$

Nano-LC/MSMS analysis of peptide libraries: Nano-LC/MSMS analysis of library bead mixtures was performed on Thermo Fisher Orbitrap Fusion Lumos Tribrid Mass Spectrometer coupled to Thermo Fisher EASY-nLC 1200 System equipped with an Acclaim PepMap RSLC C18 column (150 mm x $75 \mu \mathrm{m}$ ID, $2 \mu \mathrm{m}$ particle size, $100 \AA$ pore size) and a nanoViper C18 Trap Column ( $20 \mathrm{~mm} \times 75 \mu \mathrm{m}$ ID, $3 \mu \mathrm{m}$ particle size, $100 \AA$ pore size). The standard nano-LC method was run at $40{ }^{\circ} \mathrm{C}$ and a flow rate of $300 \mathrm{~nL} / \mathrm{min}$ with the following gradient: $1 \%$ of $80 \%$ acetonitrile in water with $0.1 \%$ formic acid added (solvent $B^{\prime}$ ) in water containing $0.1 \% \mathrm{FA}$ (solvent $A^{\prime}$ ) ramping linearly to $5 \% \mathrm{~B}^{\prime}$ in $\mathrm{A}^{\prime}$ over 2 minutes, followed by $5-61 \% \mathrm{~B}^{\prime}$ in $\mathrm{A}^{\prime}$ ramping linearly over 118 minutes, followed by 61-99\% $B^{\prime}$ in $A^{\prime}$ ramping linearly over 5 minutes and finally $99 \% B^{\prime}$ in $A^{\prime}$ for 5 minutes. $\mathrm{MS}^{2}$ acquisition over the course of the method was performed in a datadependent style (Top $N=15, z=2-10$, intensity threshold $=10^{5}$ ) with a dynamic precursor exclusion for 30 seconds after each scan. CID and HCD fragmentation 9 spectra were acquired for every selected precursor ion. Orbitrap was used as a detection method for both primary (resolution $=120000$ ) and secondary (resolution $=30000)$ mass spectra.

Purification: Normal-phase column chromatography was performed with a Biotage Selekt flash purification system, equipped with $25 \mathrm{~g}$ or $100 \mathrm{~g}$ Biotage Sfär Silica HC-D $20 \mu \mathrm{m}$ columns, using an appropriate gradient of EtOAc/Hexanes. Reversed-phase column chromatography was performed with a Biotage Selekt flash purification system equipped with either $10 \mathrm{~g}$ Biotage SNAP Bio C18 $20 \mu \mathrm{m}$ columns or 25 g Biotage Sfär Bio C18 D $20 \mu$ m columns with an appropriate gradient of $\mathrm{MeCN} / \mathrm{H}_{2} \mathrm{O}(0.1 \%$ TFA). Reversed-phase preparative HPLC was performed using an Agilent mass directed purification system (1260 infinity LC and 6130 single quad MS), equipped with an Agilent Zorbax SB C3 column $(9.4 \times 250 \mathrm{~mm}$, $5 \mu \mathrm{m})$, using an appropriate gradient of $\mathrm{MeCN} / \mathrm{H}_{2} \mathrm{O}(0.1 \% \mathrm{TFA})$. 
Preparative organic synthesis of Fmoc protected nucleobase amino acid monomers

Schemes<smiles>O=C(N[C@@H]1COC1=O)c1ccccc1</smiles>

1<smiles>O=C1OC[C@H]1NC=[18O]</smiles>

1<smiles>O=C(O)NC1COC1=O</smiles>

1<smiles>O=C(N[C@H]1COC1=O)c1ccccc1</smiles>

1

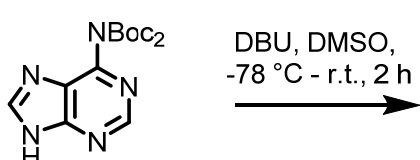

4<smiles>CCCCC(=O)OC(=O)N[C@@H](Cn1cnc2c(NC(=O)OC(C)(C)C)ncnc21)C(=O)O</smiles>
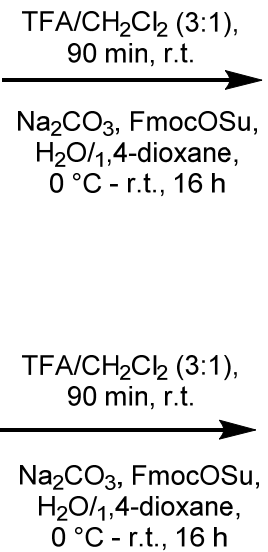<smiles>CC(C)(C)OC(=O)N[C@H](Cn1ccc(NC(=O)OCc2ccccc2)nc1=O)C(=O)O</smiles><smiles>CCC(C)C(=O)N[C@@H](Cn1cnc2c(=O)[nH]c(N)nc21)C(=O)O</smiles>

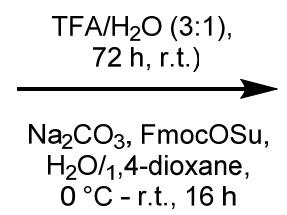

$$
\begin{gathered}
\underset{\mathrm{TFA} / \mathrm{CH}_{2} \mathrm{Cl}_{2}(3: 1),}{90 \mathrm{~min} \text {, r.t. }} \\
\underset{\mathrm{Na} \mathrm{CO}_{3}, \text { FmocOSu, }}{\mathrm{H} \mathrm{H}_{2} \mathrm{O} / 1,4 \text {-dioxane, }} \\
0{ }^{\circ} \mathrm{C}-\mathrm{rt}, 16 \mathrm{~h}
\end{gathered}
$$<smiles>Nc1ncnc2c1ncn2C[C@H](NC(F)F)C(=O)O</smiles><smiles>[Y4]C(=O)Nc1ccn(C[C@H](NC(=O)OCc2ccccc2)C(=O)O)c(=O)n1</smiles>

SI Scheme 1. Synthesis of alanyl nucleobase monomers. Protecting group discussion: Attempts to use adenine without any N6 protecting group produced both 13a and its regioisomer (7-adenilyl; data not shown). The bis-Boc protection on N6 directs the nucleophilic ring opening to the desired 9-adenilyl isomer. 13 could be used for SPPS without protecting on N6: no side reaction due to amide couplings on N6 were observed. Cytosine could be used without any N4 protecting groups: the monomer synthesis was successful and during SPPS no side reaction due to amide couplings on N4 were observed. After SPPS, however, we observed 20 - 30\% elimination of the cytosine nucleobase from the nucleopeptides. N4-Cbz protection prevented this (not further investigated) side reaction. 


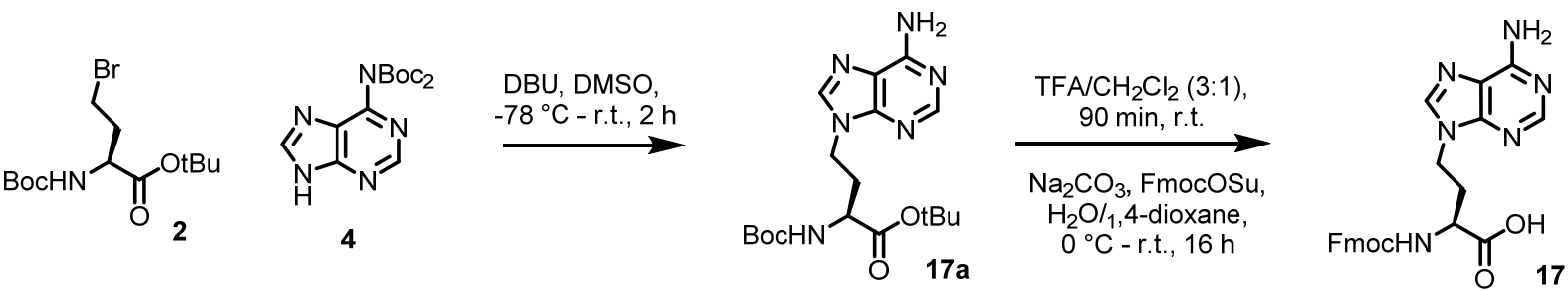

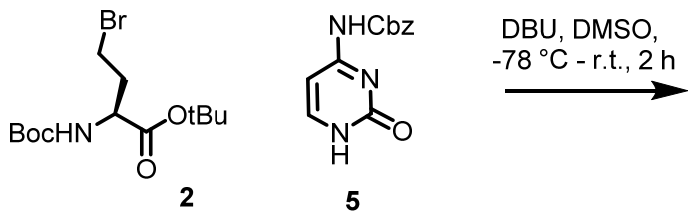<smiles>CCCCOC(=O)C(CCn1ccc(NC(=O)OCc2ccccc2)nc1=O)NC(=O)OCC(C)(C)C</smiles><smiles>CC(=O)Nc1ccn(CCC(NC(F)F)C(=O)O)c(=O)n1</smiles><smiles>CCCCOC(=O)C(CCBr)NC(=O)OCc1ccccc1</smiles>

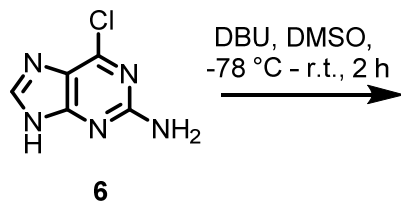<smiles>CCCCOC(=O)C(CCn1cnc2c(Cl)nc(N)nc21)NC(=O)OCc1ccccc1</smiles>

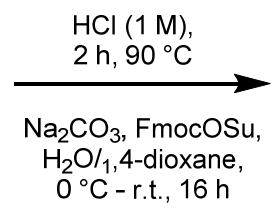<smiles>Nc1nc2c(ncn2CCC(NC(F)F)C(=O)O)c(=O)[nH]1</smiles><smiles>CCCOC(=O)C(CCBr)NC(=O)OCc1ccccc1</smiles>

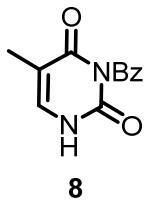

DBU, DMSO,
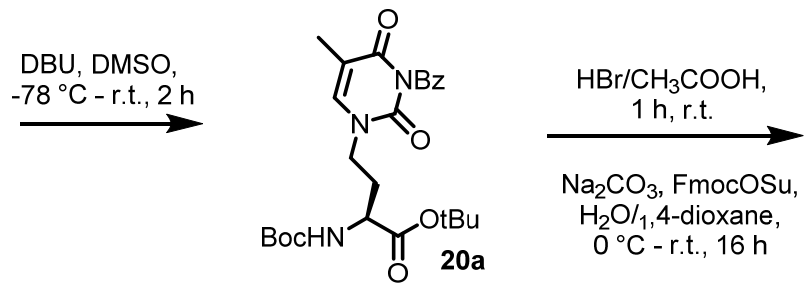<smiles>Cc1cn(CCC(NC(F)F)C(=O)O)c(=O)[nH]c1=O</smiles>

SI Scheme 2. Synthesis of homoalanyl nucleobase monomers. Protecting group discussion: Attempts to use adenine without any N6 protecting group produced both 17a and its regioisomer (7-adenilyl; data not shown). The bis-Boc protection on $\mathrm{N} 6$ directs the nucleophilic substitution to the desired 9-adenilyl isomer. 17 could be used for SPPS without protecting on N6: no side reaction due to amide couplings on N6 were observed. While cytosine could be used without any N4 protecting group for the nucleophilic substitution step, during SPPS we observed side reaction due to amide couplings on N4. Prolonged acidic cleavages or permanence in aqueous conditions removed these secondary side chains, but to avoid inefficient SPPS, we used N4-Cbz protection. Unprotected thymidine afforded > 30\% N3 regioisomer in the nucleophilic substitution. Benzoyl protection was used to generate pure 20a. 
Synthesis procedures

\section{Synthesis of 6-N(Boc) ${ }_{2}$-adenine (4)}

According to Dey et al., J. Org. Chem. 2000, 65, 7697<smiles>CC(C)(C)O[Na]</smiles>

To a solution of adenine $(2.70 \mathrm{~g}, 20.0 \mathrm{mmol})$ in THF (anhydrous, $100 \mathrm{~mL}$ ) was added Boc anhydride $(18.4 \mathrm{~mL}, 80 \mathrm{mmol}$ ) and 4-(Dimethylamino)pyridine $(244 \mathrm{mg}, 2.00 \mathrm{mmol})$. After $16 \mathrm{~h}$ stirring at room temperature the solvent was removed under reduced pressure and the mixture taken up in EtOAc $(500 \mathrm{~mL})$. The organic phase was washed with $\mathrm{HCl}(1 \mathrm{M}$, aq. sol., $100 \mathrm{~mL}$ ) and $\mathrm{NaCl}$ (sat. aq. sol., $100 \mathrm{~mL}$ ) and then dried under reduced pressure. The resulting slurry was redissolved in $\mathrm{MeOH}(200 \mathrm{~mL})$, treated with $\mathrm{NaHCO}_{3}$ (sat. aq. sol., $90 \mathrm{~mL}$ ) and stirred for $1 \mathrm{~h}$ at $50^{\circ} \mathrm{C}$. The $\mathrm{MeOH}$ was then removed under reduced pressure, the solution diluted with $\mathrm{H}_{2} \mathrm{O}$ and extracted with $\mathrm{CH}_{2} \mathrm{Cl}_{2}(3 \times 100 \mathrm{~mL})$. The organic phase was washed with $\mathrm{HCl}(1 \mathrm{M}$, aq. sol., $100 \mathrm{~mL}$ ) and $\mathrm{NaCl}$ (sat. aq. sol., $100 \mathrm{~mL}$ ) and then dried under reduced pressure affording the desired product as a colorless solid $(4.00 \mathrm{~g}, 11.9 \mathrm{mmol}, 60 \%)$.

${ }^{1} \mathrm{H}$ NMR (400 MHz, DMSO- $\left.d_{6}\right) \delta 8.81(\mathrm{~s}, 1 \mathrm{H}), 8.64(\mathrm{~s}, 1 \mathrm{H}), 1.37(\mathrm{~s}, 18 \mathrm{H})$.

$\operatorname{TLC} R_{f}$ (EtOAC) 0.59, visualized with UV light

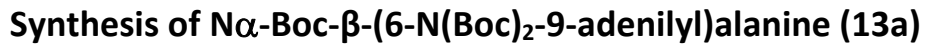<smiles>CC(C)(C)OC(=O)N[C@@H](Cn1cnc2c(N(C(C)(C)C)C(C)(C)C)ncnc21)C(=O)O</smiles>

To a solution of $4(3.00 \mathrm{~g}, 8.90 \mathrm{mmol})$ in DMSO $(60 \mathrm{~mL})$ was added DBU $(1.23 \mathrm{~g}, 8$ $\mathrm{mmol}$ ) and stirred for $2 \mathrm{~h}$ at room temperature. The solution was then cooled to -78 ${ }^{\circ} \mathrm{C}$ and Boc-serinolactone $(1.5 \mathrm{~g}, 8 \mathrm{mmol})$ dissolved in DMF $(20 \mathrm{~mL})$ was added dropwise. The reaction was allowed to warm up to room temperature and was stirred over-night. EtOAc $(250 \mathrm{~mL})$ was added to the solution and washed with aq. $\mathrm{HCl}(1 \mathrm{M}, 3 \times 50 \mathrm{~mL})$. The organic layer was dried with $\mathrm{Na}_{2} \mathrm{SO}_{4}$ and solvent removed under reduced pressure. Column chromatography over $\mathrm{SiO}_{2}$ (hexanes/EtOAc $=1: 1$ ) afforded the product as a colorless solid $(1.75 \mathrm{~g}, 3.35 \mathrm{mmol}, 38 \%)$.

${ }^{1} \mathrm{H}$ NMR (500 MHz, DMSO-d6) $\delta 13.09(\mathrm{~s}, 1 \mathrm{H}), 8.85(\mathrm{~s}, 1 \mathrm{H}), 8.49(\mathrm{~s}, 1 \mathrm{H}), 7.39-7.30(\mathrm{~m}, 1 \mathrm{H}), 4.71(\mathrm{~d}, J=$ $9.0 \mathrm{~Hz}, 1 \mathrm{H}), 4.51(\mathrm{t}, J=6.1 \mathrm{~Hz}, 2 \mathrm{H}), 1.37(\mathrm{~s}, 27 \mathrm{H})$.

${ }^{13} \mathrm{C}$ NMR $\left(126 \mathrm{MHz}\right.$, DMSO- $\left.d_{6}\right) \delta 171.63,162.77,155.65,153.70,151.87,150.45,149.41,147.52,145.56$, $128.12,83.69,83.59,78.95,53.12,44.44,36.25,31.24,28.47,27.75$. 


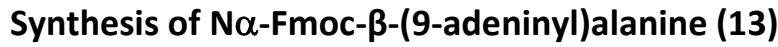<smiles>Nc1ncnc2c1ncn2C[C@H](NC(F)F)C(=O)O</smiles>

13a (1.75 g, $3.35 \mathrm{mmol})$ was dissolved in TFA/DCM (60 mL, 3:1) and stirred for 90 min at room temperature. The solvent was removed under reduced pressure and the mixture taken up in aq. $\mathrm{Na}_{2} \mathrm{CO}_{3}(10 \%, 50 \mathrm{~mL})$ and 1,4-dioxane $(20 \mathrm{~mL})$ and cooled to $0^{\circ} \mathrm{C}$. FmocOSu $(1.30 \mathrm{~g}, 4.02 \mathrm{mmol})$ was dissolved in 1,4-dioxane $(30 \mathrm{~mL})$ and added dropwise to the mixture. The reaction was allowed to reach room temperature and was stirred overnight. $\mathrm{H}_{2} \mathrm{O}(100 \mathrm{~mL})$ was added to the solution and washed with hexanes $(3 \times 50 \mathrm{~mL})$. The aqueous phase was acidified with aq. $\mathrm{HCl}(6 \mathrm{M})$ until precipitation of a colorless solid was observed. The precipitate was filtered and washed with aq. $\mathrm{HCl}$ ( 1 M), affording the desired product as a colorless solid ( $800 \mathrm{mg}, 1.80 \mathrm{mmol}, 54 \%$ over two steps).

${ }^{1}$ H NMR (400 MHz, DMSO- $\left.d_{6}\right) \delta 13.25(\mathrm{~s}, 1 \mathrm{H}), 8.46(\mathrm{~s}, 2 \mathrm{H}), 8.35(\mathrm{~s}, 1 \mathrm{H}), 8.25(\mathrm{~s}, 1 \mathrm{H}), 7.90$ (dd, J = 14.4, 7.8 $\mathrm{Hz}, 3 \mathrm{H}), 7.60(\mathrm{dd}, J=12.6,7.5 \mathrm{~Hz}, 2 \mathrm{H}), 7.41(\mathrm{td}, J=7.5,3.3 \mathrm{~Hz}, 2 \mathrm{H}), 7.36-7.27(\mathrm{~m}, 2 \mathrm{H}), 4.69-4.62(\mathrm{~m}$, $1 \mathrm{H}), 4.57-4.44(\mathrm{~m}, 2 \mathrm{H}), 4.29-4.12(\mathrm{~m}, 3 \mathrm{H})$.

${ }^{13} \mathrm{C}$ NMR $\left(101 \mathrm{MHz}\right.$, DMSO- $\left.d_{6}\right) \delta 171.31,156.30,149.57,144.14,144.10,143.56,141.15,128.12,127.54$, $125.60,125.57,120.59,118.65,66.25,53.83,46.95,44.21$.

HRMS (ESI-QTOF) $m / z:[\mathrm{M}+\mathrm{H}]^{+}$calcd 445.1546, found 445.1791 . 


\section{Synthesis of Na-Boc-(N4-Cbz-1-cytosinyl)alanine (14a)}

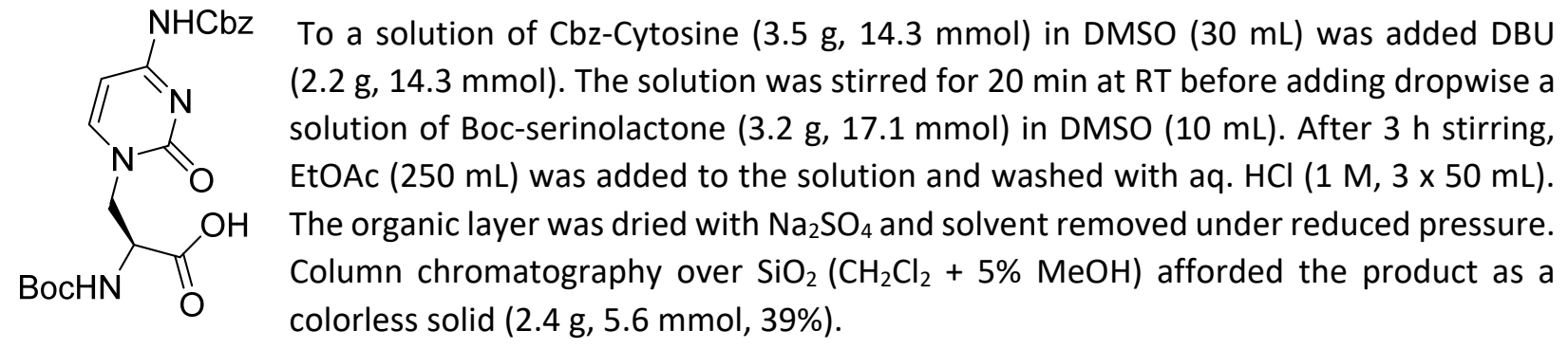

${ }^{1}$ H NMR $\left(500 \mathrm{MHz}\right.$, DMSO- $\left.d_{6}\right) \delta 7.85(\mathrm{~d}, J=7.4 \mathrm{~Hz}, 1 \mathrm{H}), 7.46-7.29(\mathrm{~m}, 6 \mathrm{H}), 7.12(\mathrm{~d}, J=8.6 \mathrm{~Hz}, 1 \mathrm{H}), 6.97$ $(\mathrm{d}, J=7.6 \mathrm{~Hz}, 1 \mathrm{H}), 5.21-5.17(\mathrm{~m}, 2 \mathrm{H}), 4.42-4.33(\mathrm{~m}, 2 \mathrm{H}), 3.71-3.61(\mathrm{~m}, 1 \mathrm{H}), 1.32(\mathrm{~d}, J=3.6 \mathrm{~Hz}, 9 \mathrm{H})$.

HRMS (ESI-QToF) $m / z:[M+H]^{+}$calcd 433.1723, found 433.1732 .

\section{Synthesis of N $\alpha$-Fmoc-(N4-Cbz-1-cytosinyl)alanine (14)}

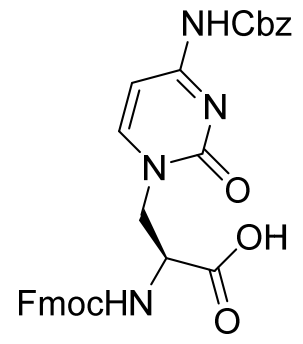

14a (2.4 g, $5.6 \mathrm{mmol})$ was dissolved in TFA/DCM (60 mL, 3:1) and stirred for $90 \mathrm{~min}$ at room temperature. The solvent was removed under reduced pressure and the mixture taken up in aq. $\mathrm{Na}_{2} \mathrm{CO}_{3}(10 \%, 50 \mathrm{~mL})$ and 1,4-dioxane $(20 \mathrm{~mL})$ and cooled to $0{ }^{\circ} \mathrm{C}$. FmocOSu $(2.00 \mathrm{~g}, 5.9 \mathrm{mmol})$ was dissolved in 1,4-dioxane $(30 \mathrm{~mL})$ and added dropwise to the mixture. The reaction was allowed to reach room temperature and was stirred overnight. $\mathrm{H}_{2} \mathrm{O}(100 \mathrm{~mL})$ was added to the solution and washed with hexanes $(3 \times 50 \mathrm{~mL})$. The aqueous phase was acidified with aq. $\mathrm{HCl}(6 \mathrm{M})$ until precipitation of a colorless solid was observed. The precipitate was filtered and washed with aq. $\mathrm{HCl}$ (1 M). The precipitate was the desired product $(1.9 \mathrm{~g}, 3.4 \mathrm{mmol}, 61 \%)$.

${ }^{1} \mathrm{H}$ NMR $\left(400 \mathrm{MHz}, \mathrm{DMSO}-d_{6}\right) \delta 10.81(\mathrm{~s}, 1 \mathrm{H}), 7.94-7.75(\mathrm{~m}, 4 \mathrm{H}), 7.63(\mathrm{t}, J=7.5 \mathrm{~Hz}, 2 \mathrm{H}), 7.48-7.26(\mathrm{~m}$, $9 \mathrm{H}), 6.94(\mathrm{~d}, J=7.3 \mathrm{~Hz}, 1 \mathrm{H}), 5.24-5.14(\mathrm{~m}, 2 \mathrm{H}), 4.49-4.36(\mathrm{~m}, 2 \mathrm{H}), 4.35-4.17(\mathrm{~m}, 3 \mathrm{H}), 3.84-3.70(\mathrm{~m}$, $1 \mathrm{H})$.

${ }^{13}$ C NMR $\left(101 \mathrm{MHz}, \mathrm{DMSO}-d_{6}\right) \delta 171.66,163.48,156.44,144.21,144.13,141.19,136.43,128.95,128.64$, $128.44,128.40,128.10,127.51,125.58,120.58,66.96,66.17,52.07,47.02$.

HRMS (ESI-QToF) $m / z:[\mathrm{M}+\mathrm{H}]^{+}$calcd 555.1880, found 555.1880 . 


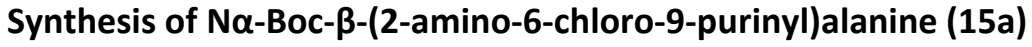<smiles>Nc1nc(Cl)c2ncn(C[C@H](NC(=O)OCc3ccccc3)C(=O)O)c2n1</smiles>

To a solution of 6-chloropurine ( $3.77 \mathrm{~g}, 22.3 \mathrm{mmol})$ in DMSO $(40 \mathrm{~mL})$ was added DBU (4.06 g, $26.6 \mathrm{mmol})$. The solution was stirred for $20 \mathrm{~min}$ at RT before adding dropwise a solution of Boc-serinolactone $(5.0 \mathrm{~g}, 26.7 \mathrm{mmol})$ in DMSO (30 mL). After $2 \mathrm{~h}$ stirring, EtOAc $(250 \mathrm{~mL})$ was added to the solution and washed with aq. $\mathrm{HCl}(1 \mathrm{M}, 3 \times 50 \mathrm{~mL})$. The organic layer was dried with $\mathrm{Na}_{2} \mathrm{SO}_{4}$ and solvent removed under reduced pressure. Column chromatography over $\mathrm{SiO}_{2}\left(\mathrm{CH}_{2} \mathrm{Cl}_{2}+\right.$ $10 \% \mathrm{MeOH}$ ) afforded the product as a colorless solid (5.0 g, $14.0 \mathrm{mmol}, 63 \%)$.

${ }^{1} \mathrm{H}$ NMR $\left(400 \mathrm{MHz}, \mathrm{DMSO}-\mathrm{d}_{6}\right) \delta 13.10(\mathrm{~s}, 1 \mathrm{H}), 7.94(\mathrm{~s}, 1 \mathrm{H}), 7.29(\mathrm{~d}, \mathrm{~J}=8.6 \mathrm{~Hz}, 1 \mathrm{H}), 6.95(\mathrm{~s}, 2 \mathrm{H}), 4.50-4.35(\mathrm{~m}, 2 \mathrm{H})$, $4.22(\mathrm{dd}, J=13.5,9.8 \mathrm{~Hz}, 1 \mathrm{H}), 1.29(\mathrm{~s}, 9 \mathrm{H})$.

${ }^{13}$ C NMR $\left(101 \mathrm{MHz}\right.$, DMSO- $\left.d_{6}\right) \delta 171.70,160.27,155.70,154.60,149.75,143.89,123.71,79.02,52.88,43.94,40.90$, $28.47,27.97$.

HRMS (SI-QToF) m/z: [M+H] calcd 357.1078, found 357.1077.

\section{Synthesis of N $\alpha-F m o c-\beta-($ (9-guaninyl)alanine (15)}<smiles>Nc1nc2c(ncn2C[C@H](NC(F)F)C(=O)O)c(=O)[nH]1</smiles>

15a (5.0 g, $14.0 \mathrm{mmol}$ ) was dissolved in TFA/ $\mathrm{H}_{2} \mathrm{O}(80 \mathrm{~mL}, 3: 1)$ and stirred for 72 $\mathrm{h}$ at room temperature. The solvent was removed under reduced pressure and the mixture taken up in aq. $\mathrm{Na}_{2} \mathrm{CO}_{3}(10 \%, 50 \mathrm{~mL})$ and 1,4-dioxane $(20 \mathrm{~mL})$ and cooled to $0{ }^{\circ} \mathrm{C}$. FmocOSu (5.7 g, $16.8 \mathrm{mmol}$ ) was dissolved in 1,4-dioxane (30 $\mathrm{mL}$ ) and added dropwise to the mixture. The reaction was allowed to reach room temperature and was stirred overnight. $\mathrm{H}_{2} \mathrm{O}(100 \mathrm{~mL})$ was added to the solution and washed with hexanes $(3 \times 50 \mathrm{~mL})$. The aqueous phase was acidified with aq. $\mathrm{HCl}(6 \mathrm{M})$ until precipitation of a colorless solid was observed. The precipitate was triturated with hexanes $/ \mathrm{CH}_{2} \mathrm{Cl}_{2}$ (1:1), centrifuged and collected, affording the desired product (4.8 g, $10.4 \mathrm{mmol}, 74 \%$ ).

${ }^{1} \mathrm{H}$ NMR $\left(400 \mathrm{MHz}\right.$, DMSO- $\left.d_{6}\right) \delta 13.19(\mathrm{~s}, 1 \mathrm{H}), 10.84(\mathrm{~s}, 1 \mathrm{H}), 7.89(\mathrm{~d}, J=7.7 \mathrm{~Hz}, 4 \mathrm{H}), 7.63(\mathrm{dd}, J=15.6,7.5$ $\mathrm{Hz}, 2 \mathrm{H}), 7.45-7.39(\mathrm{~m}, 2 \mathrm{H}), 7.37-7.27(\mathrm{~m}, 2 \mathrm{H}), 6.70(\mathrm{~s}, 2 \mathrm{H}), 4.49-4.37(\mathrm{~m}, 2 \mathrm{H}), 4.34-4.14(\mathrm{~m}, 4 \mathrm{H})$.

${ }^{13} \mathrm{C}$ NMR $\left(101 \mathrm{MHz}\right.$, DMSO- $\left.d_{6}\right) \delta 171.70,160.27,155.70,154.60,149.75,143.89,123.71,79.02,52.88$, 43.94, 40.90, 28.47, 27.97.

HRMS (SI-QToF) $m / z:[\mathrm{M}+\mathrm{H}]^{+}$calcd 461.1573, found 461.1620 . 


\section{Synthesis of N $\alpha$-Boc- $\beta$-(1-thyminyl)alanine (16a)}<smiles>Cc1cn(C[C@H](NC(=O)OCc2ccccc2)C(=O)O)c(=O)[nH]c1=O</smiles>

To a solution of Thymidine $(2.81 \mathrm{~g}, 22.3 \mathrm{mmol})$ in DMSO $(40 \mathrm{~mL})$ was added DBU (4.06 g, $26.6 \mathrm{mmol})$. The solution was stirred for $20 \mathrm{~min}$ at RT before adding dropwise a solution of Boc-serinolactone $(5.0 \mathrm{~g}, 26.7 \mathrm{mmol})$ in DMSO (30 mL). After $2 \mathrm{~h}$ stirring, EtOAc $(250 \mathrm{~mL})$ was added to the solution and washed with aq. $\mathrm{HCl}(1 \mathrm{M}, 3 \times 50 \mathrm{~mL})$. The organic layer was dried with $\mathrm{Na}_{2} \mathrm{SO}_{4}$ and solvent removed under reduced pressure. Column chromatography over $\mathrm{SiO}_{2}\left(\mathrm{CH}_{2} \mathrm{Cl}_{2}+5 \% \mathrm{MeOH}\right)$ afforded the product as a colorless solid $(2.0 \mathrm{~g}, 6.4 .0 \mathrm{mmol}, 29 \%)$.

${ }^{1} \mathrm{H}$ NMR $\left(500 \mathrm{MHz}, \mathrm{DMSO}-\mathrm{d}_{6}\right) \delta 12.97(\mathrm{~s}, 1 \mathrm{H}), 11.25(\mathrm{~s}, 1 \mathrm{H}), 7.32(\mathrm{~s}, 1 \mathrm{H}), 7.14(\mathrm{~d}, J=8.9 \mathrm{~Hz}, 1 \mathrm{H}), 4.37-4.28(\mathrm{~m}, 1 \mathrm{H})$, $4.21(\mathrm{dd}, J=13.7,4.8 \mathrm{~Hz}, 1 \mathrm{H}), 3.55(\mathrm{dd}, J=13.7,10.2 \mathrm{~Hz}, 1 \mathrm{H}), 1.73(\mathrm{~s}, 3 \mathrm{H}), 1.33(\mathrm{~s}, 9 \mathrm{H})$.

${ }^{13} \mathrm{C}$ NMR $\left(126 \mathrm{MHz}, \mathrm{DMSO}-d_{6}\right) \delta 171.76,164.76,155.76,151.33,142.42,138.18,108.25,78.93,51.92,49.03,40.90$, $28.55,28.49,28.19,12.48,12.27$.

HRMS (SI-QToF) $m / z:[\mathrm{M}+\mathrm{H}]^{+}$calcd. 314.1352, found 314.1339.

\section{Synthesis of N $\alpha$-Fmoc- $\beta$-(1-thyminyl)alanine (16)}<smiles>Cc1cn(C[C@H](NC(F)F)C(=O)O)c(=O)[nH]c1=O</smiles>

16a (2.0 g, $6.4 \mathrm{mmol})$ was dissolved in TFA/DCM (60 mL, 3:1) and stirred for 90 $\mathrm{min}$ at room temperature. The solvent was removed under reduced pressure and the mixture taken up in aq. $\mathrm{Na}_{2} \mathrm{CO}_{3}(10 \%, 50 \mathrm{~mL})$ and 1,4-dioxane $(20 \mathrm{~mL})$ and cooled to $0{ }^{\circ} \mathrm{C}$. FmocOSu $(2.6 \mathrm{~g}, 7.7 \mathrm{mmol}$ ) was dissolved in 1,4-dioxane (30 $\mathrm{mL}$ ) and added dropwise to the mixture. The reaction was allowed to reach room temperature and was stirred overnight. $\mathrm{H}_{2} \mathrm{O}(100 \mathrm{~mL})$ was added to the solution and washed with hexanes $(3 \times 50 \mathrm{~mL})$. The aqueous phase was acidified with aq. $\mathrm{HCl}(6 \mathrm{M})$ until precipitation of a colorless solid was observed. The precipitate was filtered and washed with aq. $\mathrm{HCl}(1$ M). The precipitate was the desired product $(1.75 \mathrm{~g}, 4.0 \mathrm{mmol}, 63 \%)$.

${ }^{1} \mathrm{H}$ NMR $\left(500 \mathrm{MHz}, \mathrm{DMSO}-\mathrm{d}_{6}\right) \delta 13.09(\mathrm{~s}, 1 \mathrm{H}), 11.29(\mathrm{~s}, 1 \mathrm{H}), 7.89(\mathrm{~s}, 2 \mathrm{H}), 7.78(\mathrm{~d}, J=8.9 \mathrm{~Hz}, 1 \mathrm{H}), 7.70-7.63(\mathrm{~m}, 2 \mathrm{H})$, $7.45-7.40(\mathrm{~m}, 2 \mathrm{H}), 7.38-7.36(\mathrm{~m}, 1 \mathrm{H}), 7.35-7.30(\mathrm{~m}, 2 \mathrm{H}), 4.42-4.34(\mathrm{~m}, 1 \mathrm{H}), 4.30-4.15(\mathrm{~m}, 5 \mathrm{H}), 3.63(\mathrm{dd}, J=$ $13.8,10.0 \mathrm{~Hz}, 1 \mathrm{H}), 1.61(\mathrm{~s}, 3 \mathrm{H})$.

${ }^{13} \mathrm{C}$ NMR $\left(126 \mathrm{MHz}\right.$, DMSO-d $\left.d_{6}\right) \delta 171.63,164.74,156.48,151.33,144.22,144.08,142.35,141.18,141.15,128.14$, $127.55,127.53,125.62,120.62,120.60,108.42,66.37,52.56,48.74,47.00,25.75,12.38$.

HRMS (SI-QToF) m/z: [M+H] $]^{+}$calcd. 436.1509, found 436.1545 . 


\section{Synthesis of $\mathrm{N} \alpha-B o c-\gamma-\left(6-\mathrm{N}(\mathrm{Boc})_{2}-9-\right.$-adenilyl)alanine-OtBu (17a)}<smiles></smiles>

2 (1.5 g, $4.4 \mathrm{mmol})$, bisBoc-adenine (1.85 g, $5.5 \mathrm{mmol}), \mathrm{K}_{2} \mathrm{CO}_{3}(607 \mathrm{mg}, 4.4 \mathrm{mmol})$, $\mathrm{Cs}_{2} \mathrm{CO}_{3}(1.4 \mathrm{~g}, 4.4 \mathrm{mmol})$ and tetrabuthylamonium iodide $(162 \mathrm{mg}, 0.44 \mathrm{mmol})$ were suspended in DMF $(45 \mathrm{~mL})$ and stirred at $50{ }^{\circ} \mathrm{C}$ for $16 \mathrm{~h}$. EtOAc $(300 \mathrm{~mL})$ was added to the suspension and washed with aq. $\mathrm{HCl}(1 \mathrm{M}, 2 \times 100 \mathrm{~mL}$ ) and $\mathrm{NaCl}$ (sat. aq. sol., $50 \mathrm{~mL}$ ). Automated reversed phase column chromatography afforded the desired product as a colorless solid ( $1.5 \mathrm{~g}, 2.5 \mathrm{mmol}, 58 \%)$.

${ }^{1} \mathrm{H}$ NMR $\left(500 \mathrm{MHz}, \mathrm{DMSO}-d_{6}\right) \delta 8.84(\mathrm{~s}, 1 \mathrm{H}), 8.57(\mathrm{~s}, 1 \mathrm{H}), 7.36(\mathrm{~d}, J=7.9 \mathrm{~Hz}, 1 \mathrm{H}), 4.46-4.28$ $(\mathrm{m}, 2 \mathrm{H}), 3.77-3.68(\mathrm{~m}, 1 \mathrm{H}), 2.42-2.33(\mathrm{~m}, 1 \mathrm{H}), 2.17-2.07(\mathrm{~m}, 1 \mathrm{H}), 1.43-1.34(\mathrm{~m}, 27 \mathrm{H})$.

${ }^{13} \mathrm{C}$ NMR $\left(126 \mathrm{MHz}, \mathrm{DMSO}-d_{6}\right) \delta 171.41,155.94,153.57,151.86,150.50,149.50,147.28,128.24,83.74$, $81.16,78.81,52.17,41.21,30.76,28.64,28.35,28.01,27.76$.

HRMS (SI-QToF) m/z: [M+H] ${ }^{+}$calcd. 593.3299, found 593.3199.

\section{Synthesis of N $\alpha$-Fmoc- - -(9-adenilyl)alanine (17)}<smiles></smiles>

17a (1.59 g, $2.7 \mathrm{mmol}$ ) was dissolved in TFA/DCM (60 mL, 3:1) and stirred for $90 \mathrm{~min}$ at room temperature. The solvent was removed under reduced pressure and the mixture taken up in aq. $\mathrm{Na}_{2} \mathrm{CO}_{3}(10 \%, 50 \mathrm{~mL})$ and 1,4-dioxane $(20 \mathrm{~mL})$ and cooled to $0{ }^{\circ} \mathrm{C}$. FmocOSu (1.1 g, $\left.3.24 \mathrm{mmol}\right)$ was dissolved in 1,4-dioxane $(30 \mathrm{~mL})$ and added dropwise to the mixture. The reaction was allowed to reach room temperature and was stirred overnight. $\mathrm{H}_{2} \mathrm{O}(100 \mathrm{~mL})$ was added to the solution and washed with hexanes $(3 \times 50 \mathrm{~mL})$. The aqueous phase was acidified with aq. $\mathrm{HCl}(6 \mathrm{M})$ until precipitation of a colorless solid was observed. The precipitate was filtered and washed with aq. $\mathrm{HCl}(1 \mathrm{M})$ affording the desired product $(900 \mathrm{mg}, 1.96 \mathrm{mmol}, 73 \%)$ as a colorless solid.

${ }^{1} \mathrm{H}$ NMR $\left(500 \mathrm{MHz}, \mathrm{DMSO}-d_{6}\right) \delta 8.40(\mathrm{~d}, J=21.8 \mathrm{~Hz}, 2 \mathrm{H}), 7.95-7.72(\mathrm{~m}, 5 \mathrm{H}), 7.47-7.33(\mathrm{~m}, 4 \mathrm{H}), 4.41-4.22(\mathrm{~m}, 5 \mathrm{H})$, $3.97-3.86(\mathrm{~m}, 1 \mathrm{H}), 2.47-2.33(\mathrm{~m}, 1 \mathrm{H}), 2.24-2.11(\mathrm{~m}, 1 \mathrm{H})$.

${ }^{13} \mathrm{C}$ NMR $(126 \mathrm{MHz}$, DMSO-d 6 ) $\delta 173.45,156.61,149.24,144.29,144.26,143.74,141.22,128.14,127.58,125.77$, $125.74,120.63,118.62,66.17,51.69,47.13,41.27,31.06$.

HRMS (SI-QToF) m/z: [M+H] ${ }^{+}$calcd. 459.1781, found 459.1855 . 


\section{Synthesis of $\mathrm{N} \alpha-B o c-\gamma-(4-N(\mathrm{Cbz})-1-c y t o s i n y l) a l a n i n e-O t B u(18 a)$}<smiles></smiles>

2 (1.0 g, $2.95 \mathrm{mmol})$, Cbz-cytosine (0.98 g, $2.95 \mathrm{mmol}), \mathrm{K}_{2} \mathrm{CO}_{3}(414 \mathrm{mg}, 2.95 \mathrm{mmol})$, $\mathrm{Cs}_{2} \mathrm{CO}_{3}(828 \mathrm{mg}, 2.95 \mathrm{mmol})$ and tetrabuthylamonium iodide $(111 \mathrm{mg}, 0.2 .95$ mmol) were suspended in DMF $\left(25 \mathrm{~mL}\right.$ ) and stirred at $50{ }^{\circ} \mathrm{C}$ for $16 \mathrm{~h}$. EtOAc (300 $\mathrm{mL}$ ) was added to the suspension and washed with aq. $\mathrm{HCl}(1 \mathrm{M}, 2 \times 100 \mathrm{~mL})$ and $\mathrm{NaCl}$ (sat. aq. sol., $50 \mathrm{~mL}$ ). Automated reversed phase column chromatography afforded the desired product as a colorless solid $(0.89 \mathrm{~g}, 1.77 \mathrm{mmol}, 60 \%)$.

${ }^{1}$ H NMR (400 MHz, DMSO- $\left.d_{6}\right) \delta 10.75(\mathrm{~s}, 0 \mathrm{H}), 7.97(\mathrm{~d}, J=7.3 \mathrm{~Hz}, 1 \mathrm{H}), 7.47-7.32$ $(\mathrm{m}, 5 \mathrm{H}), 7.27(\mathrm{~d}, J=7.8 \mathrm{~Hz}, 1 \mathrm{H}), 6.98(\mathrm{~d}, J=7.3 \mathrm{~Hz}, 1 \mathrm{H}), 5.19(\mathrm{~s}, 2 \mathrm{H}), 3.91-3.73(\mathrm{~m}$, $3 \mathrm{H}), 2.13-1.99(\mathrm{~m}, 1 \mathrm{H}), 1.93-1.77(\mathrm{~m}, 1 \mathrm{H}), 1.43-1.34(\mathrm{~m}, 18 \mathrm{H})$.

${ }^{13} \mathrm{C}$ NMR $\left(101 \mathrm{MHz}\right.$, DMSO- $\left.d_{6}\right) \delta 171.51,163.25,155.92,155.29,153.68,150.37,136.45,128.94,128.61$, $128.38,94.49,81.11,78.77,66.91,52.51,47.37,29.90,28.63,28.37,28.04$.

HRMS (SI-QToF) $m / z:[\mathrm{M}+\mathrm{H}]^{+}$calcd. 503.2506, found 503.2769.

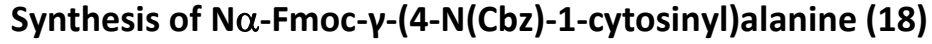<smiles></smiles>

18a (1.26 g, $2.5 \mathrm{mmol})$ was dissolved in TFA/DCM (60 mL, 3:1) and stirred for 90 min at room temperature. The solvent was removed under reduced pressure and the mixture taken up in aq. $\mathrm{Na}_{2} \mathrm{CO}_{3}(10 \%, 50 \mathrm{~mL})$ and 1,4-dioxane $(20 \mathrm{~mL})$ and cooled to $0^{\circ} \mathrm{C}$. FmocOSu (0.93 g, $2.75 \mathrm{mmol}$ ) was dissolved in 1,4-dioxane ( $30 \mathrm{~mL}$ ) and added dropwise to the mixture. The reaction was allowed to reach room temperature and was stirred overnight. $\mathrm{H}_{2} \mathrm{O}(100 \mathrm{~mL})$ was added to the solution and washed with hexanes $(3 \times 50 \mathrm{~mL})$. The aqueous phase was acidified with aq. $\mathrm{HCl}(6 \mathrm{M})$ until precipitation of a colorless solid was observed. The precipitate was filtered and washed with aq. $\mathrm{HCl}(1 \mathrm{M})$. The precipitate was the desired product $(630 \mathrm{mg}, 1.05 \mathrm{mmol}$, $42 \%)$.

${ }^{1} \mathrm{H}$ NMR $\left(400 \mathrm{MHz}\right.$, DMSO- $\left.d_{6}\right) \delta 7.99-7.85(\mathrm{~m}, 4 \mathrm{H}), 7.77-7.69(\mathrm{~m}, 3 \mathrm{H}), 7.48-7.32(\mathrm{~m}, 10 \mathrm{H}), 6.98(\mathrm{~d}, J=$ $7.2 \mathrm{~Hz}, 1 \mathrm{H}), 5.18(\mathrm{~s}, 2 \mathrm{H}), 4.83(\mathrm{~d}, J=6.1 \mathrm{~Hz}, 1 \mathrm{H}), 4.45-4.19(\mathrm{~m}, 3 \mathrm{H}), 4.00-3.91(\mathrm{~m}, 1 \mathrm{H}), 3.91-3.78(\mathrm{~m}$, $2 \mathrm{H}), 2.24-2.11(\mathrm{~m}, 1 \mathrm{H}), 2.01-1.89(\mathrm{~m}, 1 \mathrm{H})$.

${ }^{13} \mathrm{C}$ NMR $\left(101 \mathrm{MHz}\right.$, DMSO-d $\left.d_{6}\right) \delta 173.62,170.17,163.22,156.60,155.27,153.66,151.58,150.42,144.28,143.10$, 141.30, 141.21, 136.42, 128.94, 128.61, 128.43, 128.40, 128.12, 127.77, 127.57, 127.55, 125.75, 125.69, 125.37, $120.77,120.61,120.58,94.46,72.34,66.94,66.13,51.93,47.54,47.14,46.43,30.02,25.75$.

HRMS (SI-QToF) $m / z:[M+H]^{+}$calcd. 596.2036, found 569.2147. 


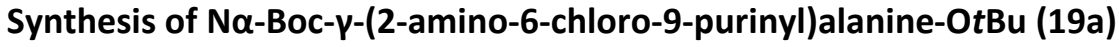<smiles>CC(C)(C)OC(=O)N[C@@H](CCn1cnc2c(Cl)nc(N)nc21)C(=O)OC(C)(C)C</smiles>

2 (1.5 g, $4.4 \mathrm{mmol}$ ), 6-chloropurine (935 mg, $5.5 \mathrm{mmol}), \mathrm{K}_{2} \mathrm{CO}_{3}(607 \mathrm{mg}, 4.4$ $\mathrm{mmol}), \mathrm{Cs}_{2} \mathrm{CO}_{3}(1.4 \mathrm{~g}, 4.4 \mathrm{mmol}$ ) and tetrabuthylamonium iodide (162 mg, 0.44 $\mathrm{mmol}$ ) were suspended in DMF $(45 \mathrm{~mL})$ and stirred at $50{ }^{\circ} \mathrm{C}$ for $16 \mathrm{~h}$. EtOAc (300 $\mathrm{mL}$ ) was added to the suspension and washed with aq. $\mathrm{HCl}(1 \mathrm{M}, 2 \times 100 \mathrm{~mL})$ and $\mathrm{NaCl}$ (sat. aq. sol., $50 \mathrm{~mL}$ ). Automated reversed phase column chromatography afforded the desired product as a colorless solid $(1.32 \mathrm{~g}, 3.08$ $\mathrm{mmol}, 70 \%)$.

${ }^{1} \mathrm{H}$ NMR $\left(500 \mathrm{MHz}, \mathrm{DMSO}-d_{6}\right) \delta 8.04(\mathrm{~s}, 1 \mathrm{H}), 7.32(\mathrm{~d}, J=7.9 \mathrm{~Hz}, 1 \mathrm{H}), 6.95(\mathrm{~d}, J=7.6 \mathrm{~Hz}$, $2 \mathrm{H}), 4.11(\mathrm{t}, J=7.1 \mathrm{~Hz}, 2 \mathrm{H}), 3.80-3.69(\mathrm{~m}, 1 \mathrm{H}), 2.34-2.21(\mathrm{~m}, 1 \mathrm{H}), 2.09-1.97(\mathrm{~m}, 1 \mathrm{H}), 1.38(\mathrm{~d}, J=20.0 \mathrm{~Hz}, 18 \mathrm{H})$.

${ }^{13} \mathrm{C}$ NMR $\left(126 \mathrm{MHz}\right.$, DMSO- $\left.d_{6}\right) \delta 171.45,160.22,155.96,154.52,149.78,143.60,123.79,81.17,78.84,52.37,40.76$, $30.44,28.63,28.34,28.02$.

HRMS (SI-QToF) $m / z:[\mathrm{M}+\mathrm{H}]^{+}$calcd. 427.1861, found 427.1785 .

\section{Synthesis of Na-Fmoc- $\gamma$-(9-guaninyl)alanine (19)}<smiles></smiles>

19a $(1.3 \mathrm{~g}, 3.0 \mathrm{mmol})$ was dissolved in aq. $\mathrm{HCl}(1 \mathrm{M}, 30 \mathrm{~mL})$ and stirred for $2 \mathrm{~h}$ at 90 ${ }^{\circ} \mathrm{C}$. The solvent was removed under reduced pressure and the mixture taken up in aq. $\mathrm{Na}_{2} \mathrm{CO}_{3}(10 \%, 50 \mathrm{~mL})$ and 1,4-dioxane $(20 \mathrm{~mL})$ and cooled to $0{ }^{\circ} \mathrm{C}$. FmocOSu (1.2 $\mathrm{g}, 3.6 \mathrm{mmol})$ was dissolved in 1,4-dioxane $(30 \mathrm{~mL})$ and added dropwise to the mixture. The reaction was allowed to reach room temperature and was stirred overnight. $\mathrm{H}_{2} \mathrm{O}(100 \mathrm{~mL})$ was added to the solution and washed with hexanes $(3 \mathrm{x}$ $50 \mathrm{~mL}$ ). The aqueous phase was acidified with aq. $\mathrm{HCl}(6 \mathrm{M})$ until precipitation of a colorless solid was observed. The precipitate was triturated with hexanes $/ \mathrm{CH}_{2} \mathrm{Cl}_{2}$ (1:1), centrifuged and collected, affording the desired product (600 mg, $1.26 \mathrm{mmol}, 42 \%$ ).

${ }^{1} \mathrm{H}$ NMR $\left(500 \mathrm{MHz}, \mathrm{DMSO}-d_{6}\right) \delta 12.82(\mathrm{~s}, 1 \mathrm{H}), 11.40(\mathrm{~s}, 1 \mathrm{H}), 8.55(\mathrm{~s}, 1 \mathrm{H}), 7.91(\mathrm{~d}, J=7.5 \mathrm{~Hz}, 2 \mathrm{H}), 7.82(\mathrm{~d}, J$ $=8.3 \mathrm{~Hz}, 1 \mathrm{H}), 7.74(\mathrm{dd}, J=7.5,2.9 \mathrm{~Hz}, 2 \mathrm{H}), 7.48-7.39(\mathrm{~m}, 2 \mathrm{H}), 7.39-7.28(\mathrm{~m}, 2 \mathrm{H}), 7.07(\mathrm{~s}, 2 \mathrm{H}), 4.35-$ $4.30(\mathrm{~m}, 2 \mathrm{H}), 4.26(\mathrm{t}, J=7.0 \mathrm{~Hz}, 1 \mathrm{H}), 4.16-4.10(\mathrm{~m}, 2 \mathrm{H}), 4.01-3.95(\mathrm{~m}, 1 \mathrm{H}), 2.41-2.31(\mathrm{~m}, 1 \mathrm{H}), 2.17-$ $2.06(\mathrm{~m}, 1 \mathrm{H})$.

${ }^{13} \mathrm{C}$ NMR $\left(126 \mathrm{MHz}, \mathrm{DMSO}-d_{6}\right) \delta 173.40,156.61,155.37,150.73,144.31,144.22,141.21,137.63,128.15,127.58$, $125.75,120.63,66.22,51.73,47.11,41.74,30.47$.

HRMS (SI-QToF) m/z: [M+H] calcd. 475.1730, found 475.1770. 


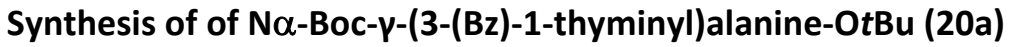<smiles>CCCCC(=O)OCCCn1cc(C)c(=O)n(CCC(NC(=O)OC(C)(C)C)C(=O)OC(C)(C)C)c1=O</smiles>

2 (1.5 g, $4.4 \mathrm{mmol})$, benzoyl-thymidine (1.27 g, $5.5 \mathrm{mmol}), \mathrm{K}_{2} \mathrm{CO}_{3}(607 \mathrm{mg}, 4.4$ $\mathrm{mmol}), \mathrm{Cs}_{2} \mathrm{CO}_{3}(1.4 \mathrm{~g}, 4.4 \mathrm{mmol}$ ) and tetrabuthylamonium iodide (162 $\mathrm{mg}, 0.44$ $\mathrm{mmol})$ were suspended in DMF $(45 \mathrm{~mL})$ and stirred at $50^{\circ} \mathrm{C}$ for $16 \mathrm{~h}$. EtOAc $(300 \mathrm{~mL})$ was added to the suspension and washed with aq. $\mathrm{HCl}(1 \mathrm{M}, 2 \times 100 \mathrm{~mL})$ and $\mathrm{NaCl}$ (sat. aq. sol., $50 \mathrm{~mL}$ ). Automated reversed phase column chromatography afforded the desired product as a colorless solid (1.80 g, $3.52 \mathrm{mmol}, 80 \%)$.

HRMS (SI-QToF) m/z: [M+Na] ${ }^{+}$calcd. 510.2216, found 510.1931.

\section{Synthesis of $\mathrm{N} \alpha$-Fmoc- $\nu$-(1-thyminyl)alanine (20)}<smiles>Cc1cn(CC[C@H](NC(F)F)C(=O)O)c(=O)[nH]c1=O</smiles>

20a (1.66 g, $3.5 \mathrm{mmol}$ ) was dissolved in $\mathrm{HBr}(30 \%$ in $\mathrm{AcOH}, 25 \mathrm{~mL}$ ) and stirred for $1 \mathrm{~h}$, at room temperature. The solvent was removed under reduced pressure and the mixture taken up in aq. $\mathrm{Na}_{2} \mathrm{CO}_{3}(10 \%, 50 \mathrm{~mL})$ and 1,4-dioxane $(20 \mathrm{~mL})$ and cooled to $0{ }^{\circ} \mathrm{C}$. FmocOSu (1.4 g, $4.2 \mathrm{mmol}$ ) was dissolved in 1,4-dioxane ( $30 \mathrm{~mL}$ ) and added dropwise to the mixture. The reaction was allowed to reach room temperature and was stirred overnight. $\mathrm{H}_{2} \mathrm{O}(100 \mathrm{~mL})$ was added to the solution and washed with hexanes $(3 \times 50 \mathrm{~mL})$. The aqueous phase was acidified with aq. $\mathrm{HCl}(6 \mathrm{M})$ until precipitation of a colorless solid was observed. The precipitate was triturated with hexanes $/ \mathrm{CH}_{2} \mathrm{Cl}_{2}$ (1:1), centrifuged and collected, affording the desired product (1.1 $\mathrm{g}$, $2.4 \mathrm{mmol}, 70 \%)$.

${ }^{1} \mathrm{H}$ NMR $\left(500 \mathrm{MHz}, \mathrm{DMSO}-d_{6}\right) \delta 12.74(\mathrm{~s}, 1 \mathrm{H}), 11.24(\mathrm{~s}, 1 \mathrm{H}), 7.91(\mathrm{~d}, J=7.5 \mathrm{~Hz}, 2 \mathrm{H}), 7.74(\mathrm{dd}, J=8.3,2.6 \mathrm{~Hz}, 2 \mathrm{H}), 7.48$ $-7.39(\mathrm{~m}, 3 \mathrm{H}), 7.35(\mathrm{td}, J=7.4,4.4 \mathrm{~Hz}, 2 \mathrm{H}), 4.37-4.23(\mathrm{~m}, 3 \mathrm{H}), 3.97(\mathrm{~m}, 1 \mathrm{H}), 3.76-3.64(\mathrm{~m}, 2 \mathrm{H}), 2.09(\mathrm{~m}, 1 \mathrm{H}), 1.96$ $-1.85(\mathrm{~m}, 1 \mathrm{H}), 1.73(\mathrm{~s}, 3 \mathrm{H})$.

${ }^{13} \mathrm{C}$ NMR $\left(126 \mathrm{MHz}, \mathrm{DMSO}-d_{6}\right) \delta 173.66,164.75,156.57,151.31,144.28,141.84,141.21,128.14,127.59,125.76$, $125.71,120.63,120.61,108.93,66.17,51.88,47.14,45.14,30.27,12.43$.

HRMS (SI-QToF) m/z: [M+Na] ${ }^{+}$calcd. 450.1665, found 450.1665 . 


\section{N $\alpha$-Fmoc-Np-(6(Bhoc)-9-adenilylacetyl))-diaminobutyric acid (21)}<smiles>CCCCCCCNc1ncnc2c1ncn2CC(=O)NCC[C@H](NC(F)F)C(=O)O</smiles>

To a solution of Bhoc-Adenine-acetic acid $(1.6 \mathrm{~g}, 4 \mathrm{mmol})$ in DMF $(20 \mathrm{~mL})$ were added TSTU ( $4 \mathrm{mmol}, 1.2 \mathrm{~g}$ ), and DIEA ( $16 \mathrm{mmol}, 700 \mu \mathrm{L})$. The solution was stirred for $15 \mathrm{~min}$ at room temperature before adding Fmoc-Dap-OH (1.53 g, $4 \mathrm{mmol})$. After $2 \mathrm{~h}$ stirring at room temperature the solvent was removed under reduced pressure and reversed phase column chromatography (120 g C18, 35-100\% MeCN in $\mathrm{H}_{2} \mathrm{O}$ over $40 \mathrm{~min}$ ) afforded the desired product as a colorless solid (800 $\mathrm{mg}$, $1.1 \mathrm{mmol}, 28 \%)$.

${ }^{1} \mathrm{H}$ NMR $\left(400 \mathrm{MHz}\right.$, DMSO- $\left.d_{6}\right) \delta 12.68(\mathrm{~s}, 1 \mathrm{H}), 10.90(\mathrm{~s}, 1 \mathrm{H}), 8.59(\mathrm{~s}, 1 \mathrm{H}), 8.40(\mathrm{~s}, 1 \mathrm{H}), 7.89$ (d, $J=7.5 \mathrm{~Hz}, 2 \mathrm{H}), 7.76-7.67(\mathrm{~m}, 3 \mathrm{H}), 7.54(\mathrm{~d}, J=7.2 \mathrm{~Hz}, 4 \mathrm{H}), 7.43-7.28(\mathrm{~m}, 14 \mathrm{H}), 6.83$ $(\mathrm{s}, 1 \mathrm{H}), 4.32-4.18(\mathrm{~m}, 3 \mathrm{H}), 3.23-3.14(\mathrm{~m}, 2 \mathrm{H}), 1.99-1.88(\mathrm{~m}, 1 \mathrm{H}), 1.85-1.71(\mathrm{~m}, 1 \mathrm{H})$.

${ }^{13} \mathrm{C}$ NMR $\left(101 \mathrm{MHz}\right.$, DMSO- $\left.d_{6}\right) \delta$ 174.12, 166.43, 156.62, 152.55, 151.86, 151.68, 149.49, 145.75, 144.29, $144.26,141.31$, 141.17, 129.29, 128.95, 128.51, 128.19, 128.10, 127.55, 127.15, 127.06, 126.96, 126.68, $125.77,125.73,122.61,120.58,77.80,74.70,66.13,52.10,47.09,45.70,36.49,30.83$.

HRMS (SI-QToF) m/z: [M+H] calcd. 726.2676, found 726.2683 .

\section{N $\alpha$-Fmoc-Np-(4(Bhoc)-1-cytosinylacetyl))-diaminobutyric acid (22)}

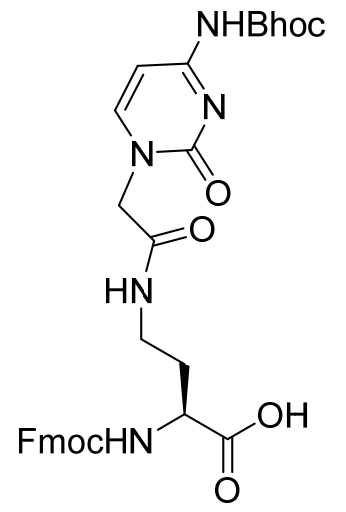

To a solution of Bhoc-C-acetic acid $(1.5 \mathrm{~g}, 4 \mathrm{mmol})$ in DMF $(20 \mathrm{~mL})$ were added TSTU $(4 \mathrm{mmol}, 1.2 \mathrm{~g})$, and DIEA ( $16 \mathrm{mmol}, 700 \mu \mathrm{L})$. The solution was stirred for $15 \mathrm{~min}$ at room temperature before adding Fmoc-Dap-OH (1.53 g, 4 mmol). After $2 \mathrm{~h}$ stirring at room temperature the solvent was removed under reduced pressure and reversed phase column chromatography (120 g C18, 35-100\% MeCN in $\mathrm{H} 2 \mathrm{O}$ over $40 \mathrm{~min}$ ) afforded the desired product as a colorless solid (610 mg, $0.87 \mathrm{mmol}, 22 \%$ ).

${ }^{1} \mathrm{H}$ NMR $\left(400 \mathrm{MHz}, \mathrm{DMSO}-\mathrm{d}_{6}\right) \delta 12.65(\mathrm{~s}, 1 \mathrm{H}), 10.96(\mathrm{~s}, 1 \mathrm{H}), 8.24(\mathrm{t}, J=5.4 \mathrm{~Hz}, 1 \mathrm{H}), 7.95(\mathrm{~d}$, $J=7.3 \mathrm{~Hz}, 1 \mathrm{H}), 7.89(\mathrm{~d}, J=7.5 \mathrm{~Hz}, 2 \mathrm{H}), 7.77-7.65(\mathrm{~m}, 3 \mathrm{H}), 7.50-7.29(\mathrm{~m}, 16 \mathrm{H}), 6.93(\mathrm{~d}, J=$ $7.3 \mathrm{~Hz}, 1 \mathrm{H}), 6.80(\mathrm{~s}, 1 \mathrm{H}), 4.35-4.19(\mathrm{~m}, 3 \mathrm{H}), 4.02(\mathrm{dq}, J=9.4,6.2,4.7 \mathrm{~Hz}, 1 \mathrm{H}), 3.25-3.08$ $(\mathrm{m}, 2 \mathrm{H}), 1.96-1.86(\mathrm{~m}, 1 \mathrm{H}), 1.85-1.70(\mathrm{~m}, 1 \mathrm{H})$.

${ }^{13} \mathrm{C}$ NMR $\left(101 \mathrm{MHz}, \mathrm{DMSO}-d_{6}\right) \delta$ 174.09, 167.05, 163.47, 156.60, 144.26, 141.17, 140.87, 129.06, 129.02, 128.31, $128.10,127.56,126.90,125.74,120.57,94.07,77.87,66.15,55.38,52.18,51.78,47.09,36.42,31.16$.

HRMS (SI-QToF) $m / z:[\mathrm{M}+\mathrm{H}]^{+}$calcd. 702.2564, found 702.2492. 


\section{N $\alpha$-Fmoc-Ny-(4(Bhoc)-9-guanidylacetyl))-diaminobutyric acid (23)}

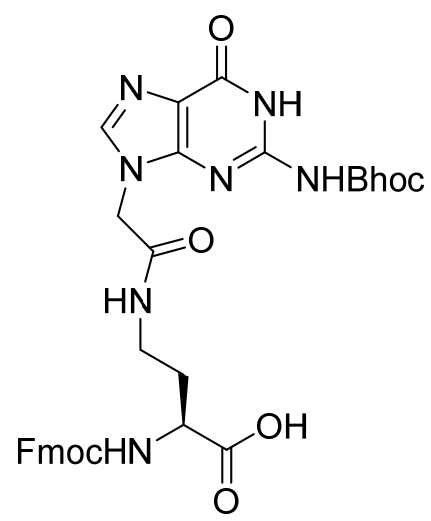

To a solution of Bhoc-G-acetic acid $(1.7 \mathrm{~g}, 4 \mathrm{mmol})$ in DMF $(20 \mathrm{~mL})$ were added TSTU ( $4 \mathrm{mmol}, 1.2 \mathrm{~g}$ ), and DIEA (16 mmol, $700 \mu \mathrm{L})$. The solution was stirred for $15 \mathrm{~min}$ at room temperature before adding Fmoc-Dap-OH (1.53 $\mathrm{g}, 4 \mathrm{mmol})$. After $2 \mathrm{~h}$ stirring at room temperature the solvent was removed under reduced pressure and reversed phase column chromatography (120 g $\mathrm{C} 18,35-100 \% \mathrm{MeCN}$ in $\mathrm{H} 2 \mathrm{O}$ over $40 \mathrm{~min}$ ) afforded the desired product as a colorless solid (750 mg, $1.0 \mathrm{mmol}, 25 \%$ ).

${ }^{1} \mathrm{H}$ NMR (400 MHz, DMSO- $\left.d_{6}\right) \delta 11.23(\mathrm{~s}, 1 \mathrm{H}), 8.36-8.27(\mathrm{~m}, 1 \mathrm{H}), 7.96(\mathrm{~s}, 1 \mathrm{H}), 7.93$ $-7.87(\mathrm{~m}, 3 \mathrm{H}), 7.76-7.67(\mathrm{~m}, 3 \mathrm{H}), 7.49-7.29(\mathrm{~m}, 14 \mathrm{H}), 6.86(\mathrm{~s}, 1 \mathrm{H}), 4.32-4.19(\mathrm{~m}$, $3 \mathrm{H}), 4.08-3.99(\mathrm{~m}, 1 \mathrm{H}), 3.63-3.53(\mathrm{~m}, 1 \mathrm{H}), 3.24-3.05(\mathrm{~m}, 3 \mathrm{H}), 2.01-1.88(\mathrm{~m}$,

$1 \mathrm{H}), 1.83-1.71(\mathrm{~m}, 1 \mathrm{H})$.

${ }^{13} \mathrm{C}$ NMR $\left(101 \mathrm{MHz}\right.$, DMSO-d $\left.d_{6}\right) \delta$ 174.13, 166.44, 156.70, 155.40, 154.16, 149.66, 147.61, 144.26, 141.18, 141.08, $140.47,129.05,128.51,128.44,128.12,127.56,127.14,127.00,126.90,126.88,126.68,125.74,120.59,119.38$, $78.51,66.17,52.07,47.08,45.72,36.47,30.81$.

HRMS (SI-QToF) $m / z:[\mathrm{M}+\mathrm{H}]^{+}$calcd. 742.2625, found 742.2654.

\section{N $\alpha$-Fmoc-Np-(thymidylacetyl))-diaminobutyric acid (24)}

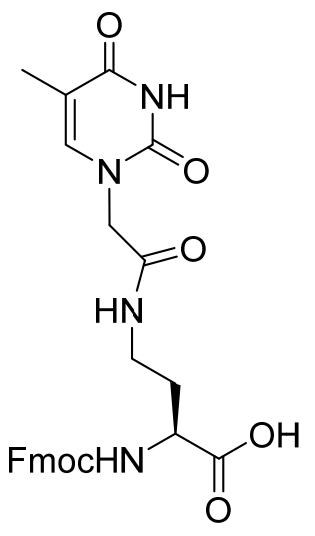

To a solution of T-acetic acid $(736 \mathrm{mg}, 4 \mathrm{mmol})$ in DMF $(20 \mathrm{~mL})$ were added TSTU ( $4 \mathrm{mmol}, 1.2 \mathrm{~g}$ ), and DIEA ( $16 \mathrm{mmol}, 700 \mu \mathrm{L})$. The solution was stirred for $15 \mathrm{~min}$ at room temperature before adding Fmoc-Dap-OH ( $1.53 \mathrm{~g}, 4 \mathrm{mmol})$. After $2 \mathrm{~h}$ stirring at room temperature the solvent was removed under reduced pressure and reversed phase column chromatography ( 120 g C18, 35-100\% MeCN in H2O over 40 $\mathrm{min}$ ) afforded the desired product as a colorless solid (550 mg, $1.1 \mathrm{mmol}, 28 \%$ ).

${ }^{1} \mathrm{H}$ NMR $\left(400 \mathrm{MHz}\right.$, DMSO-d $\left.d_{6}\right) 12.68(\mathrm{~s}, 1 \mathrm{H}), 11.26(\mathrm{~s}, 1 \mathrm{H}), 8.18(\mathrm{t}, J=5.5 \mathrm{~Hz}, 1 \mathrm{H}), 8.01-$ $7.84(\mathrm{~m}, 3 \mathrm{H}), 7.79-7.62(\mathrm{~m}, 3 \mathrm{H}), 7.49-7.38(\mathrm{~m}, 3 \mathrm{H}), 7.37-7.29(\mathrm{~m}, 2 \mathrm{H}), 4.35-4.20(\mathrm{~m}$, $5 \mathrm{H}), 4.07-3.95(\mathrm{~m}, 1 \mathrm{H}), 3.22-3.11(\mathrm{~m}, 2 \mathrm{H}), 1.98-1.86(\mathrm{~m}, 1 \mathrm{H}), 1.81-1.70(\mathrm{~m}, 4 \mathrm{H})$.

HRMS (SI-QToF) $m / z:[\mathrm{M}+\mathrm{H}]^{+}$calcd. 507.1880, found 507.1875. 
NMR spectra

\section{${ }^{1}$ H NMR (4)}

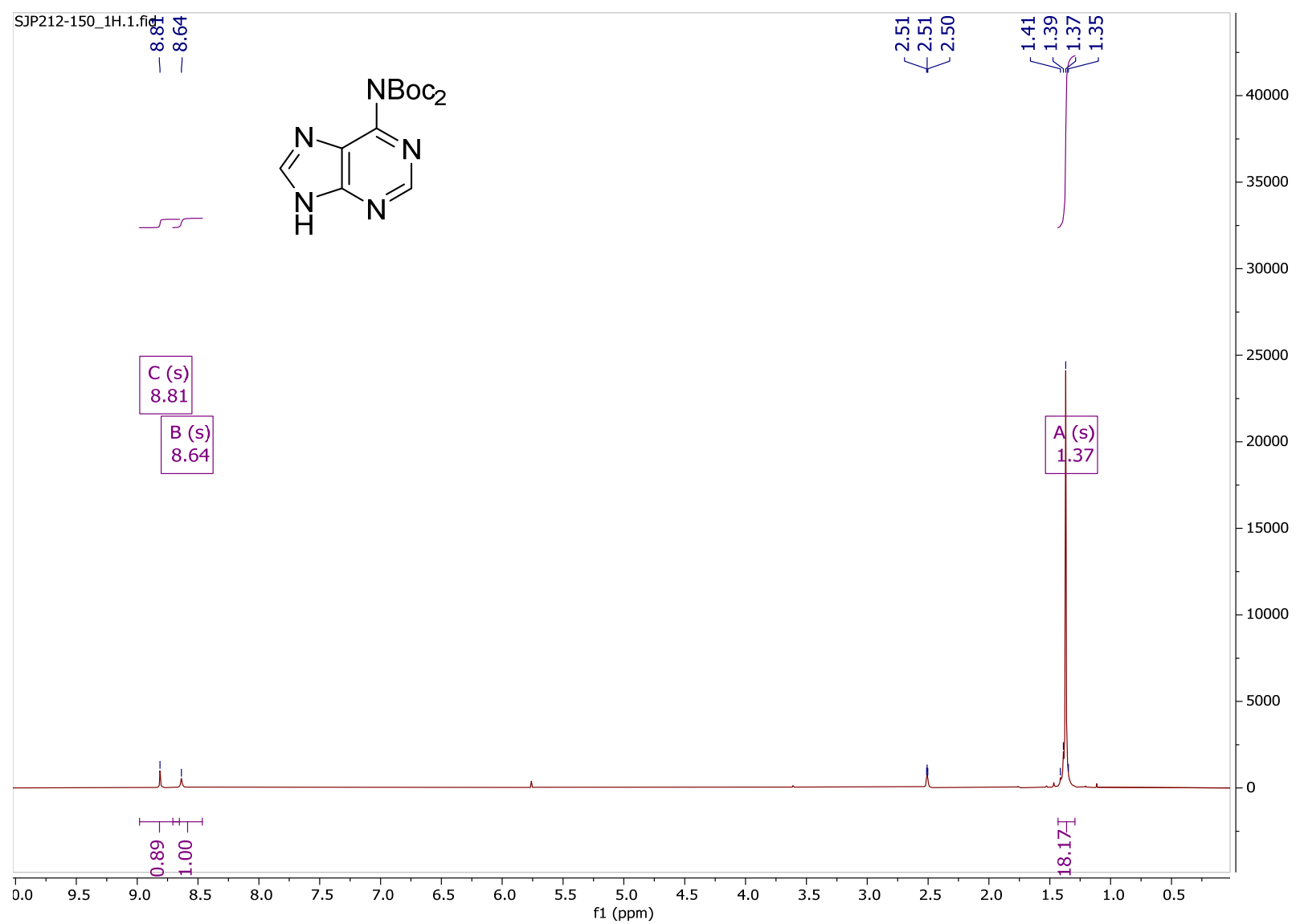




\section{${ }^{1} \mathrm{H}$ NMR (13a)}

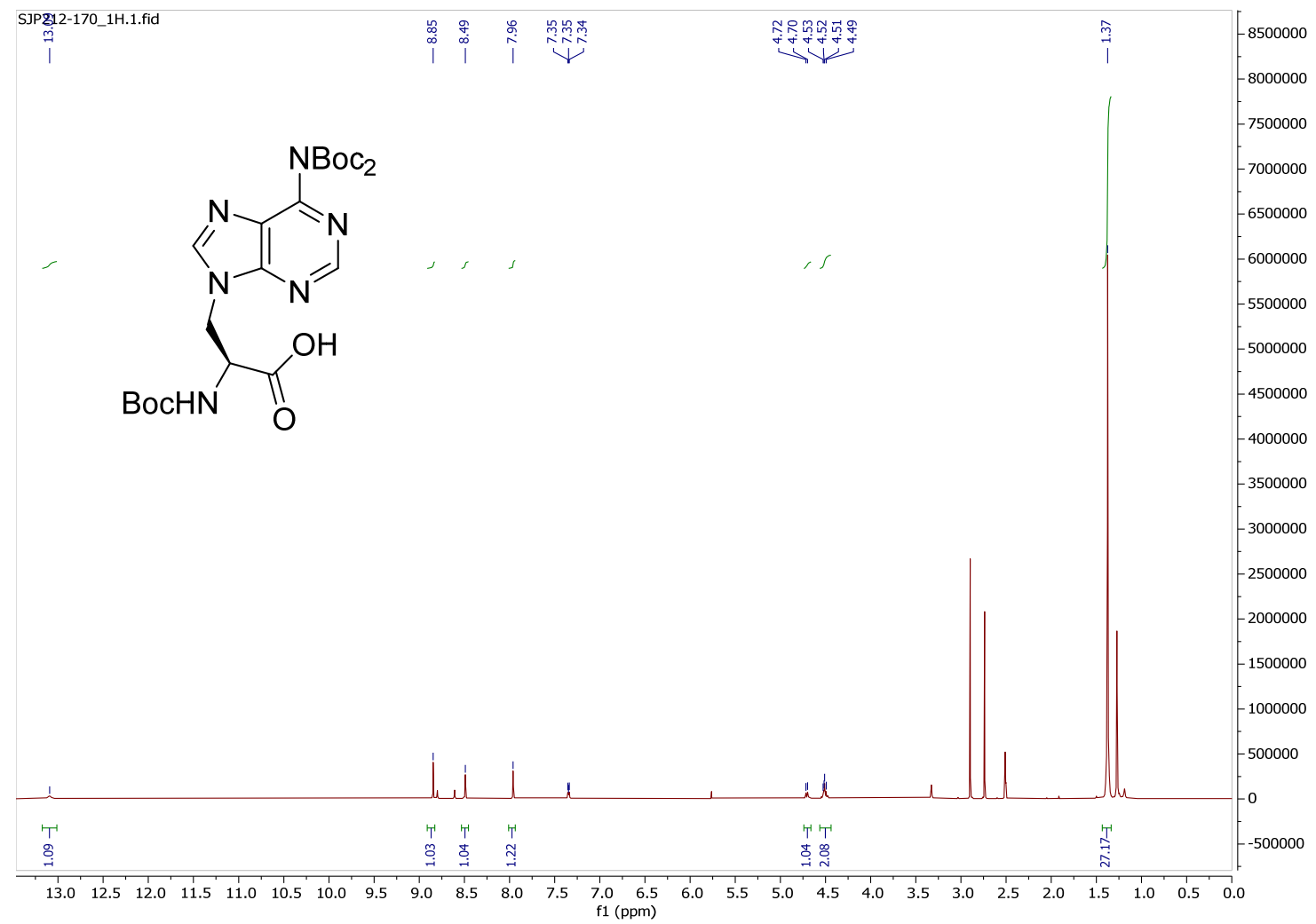

\section{${ }^{13}$ C NMR (13a)}

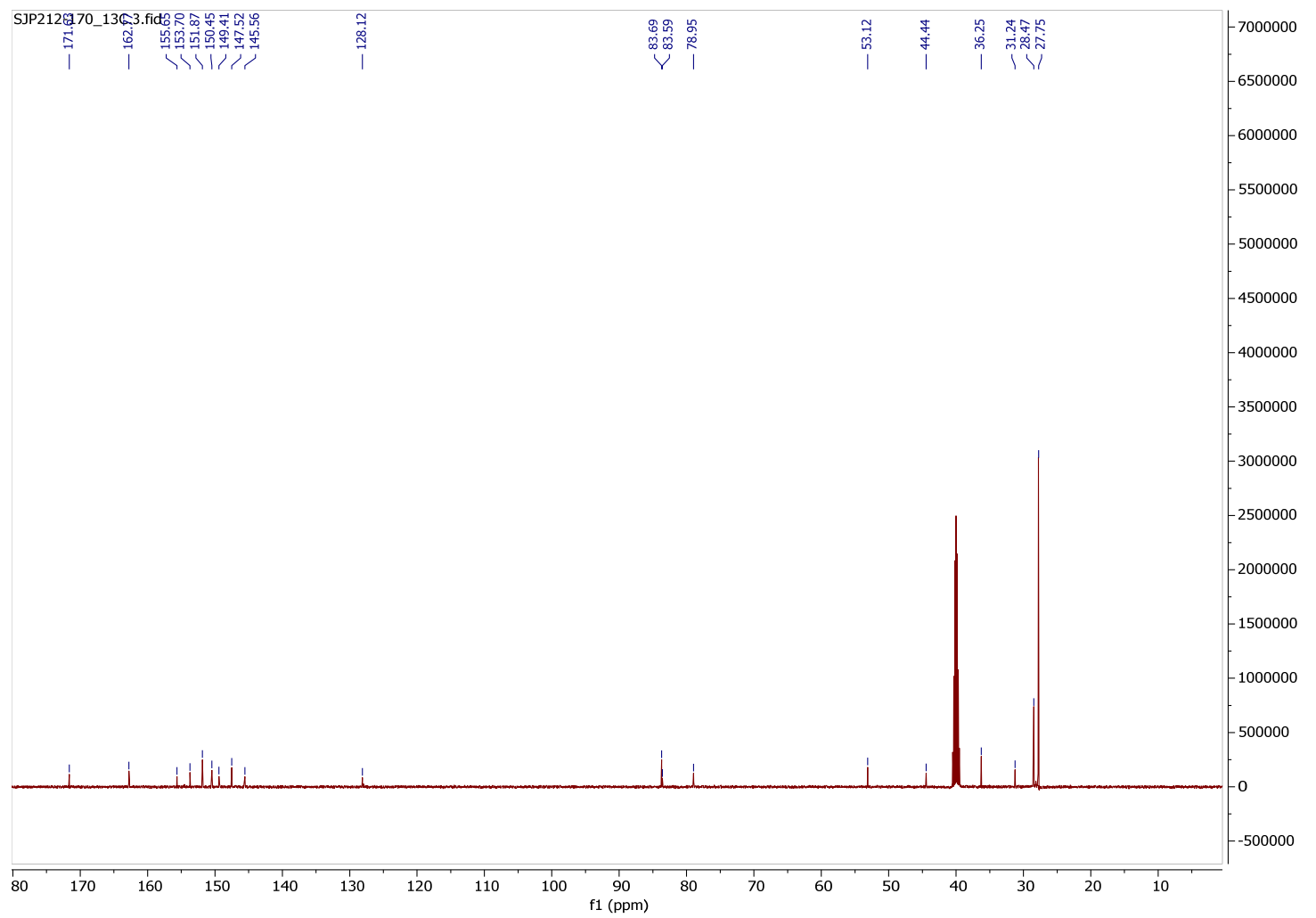




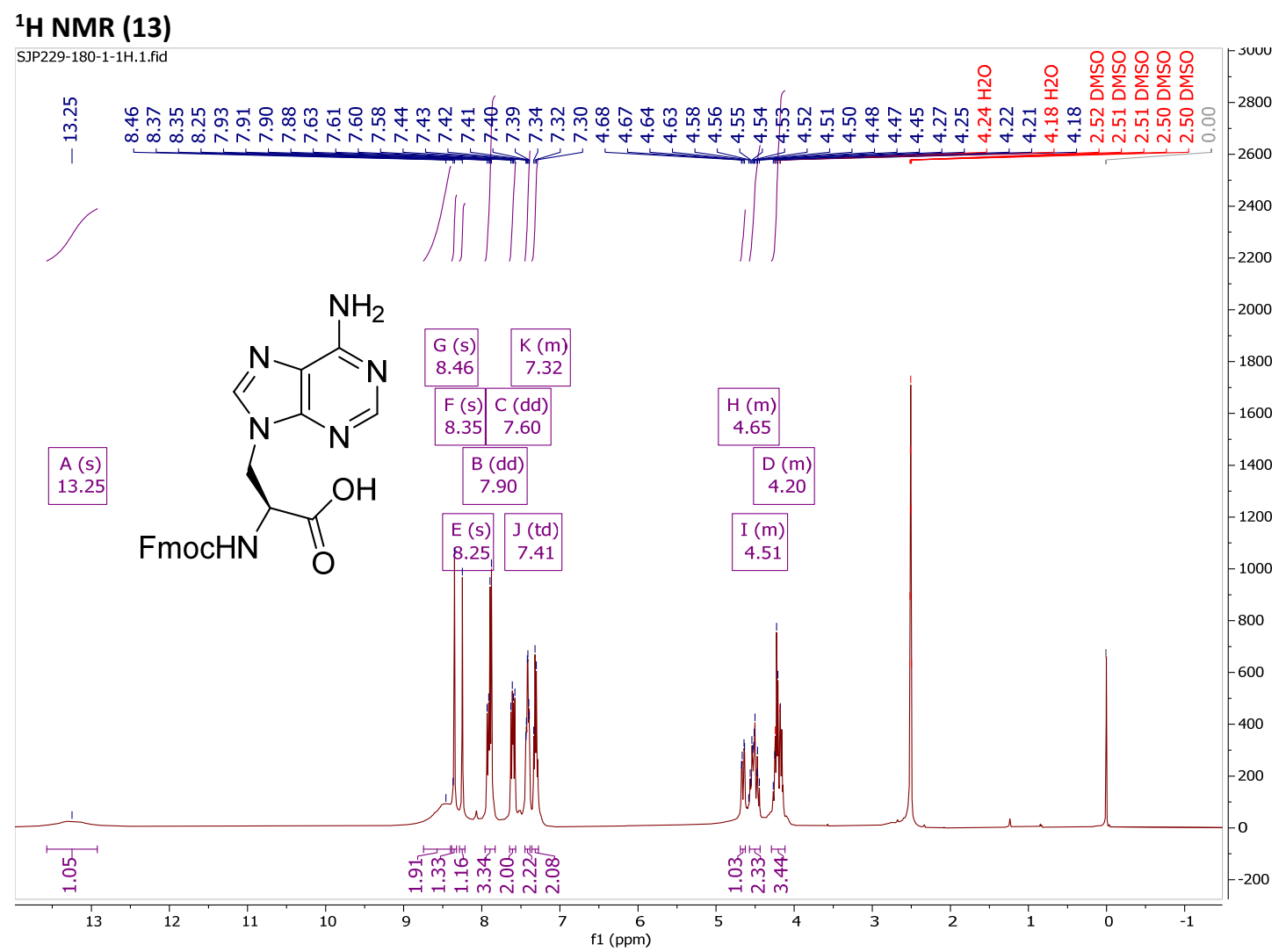

\section{${ }^{13}$ C NMR (13)}

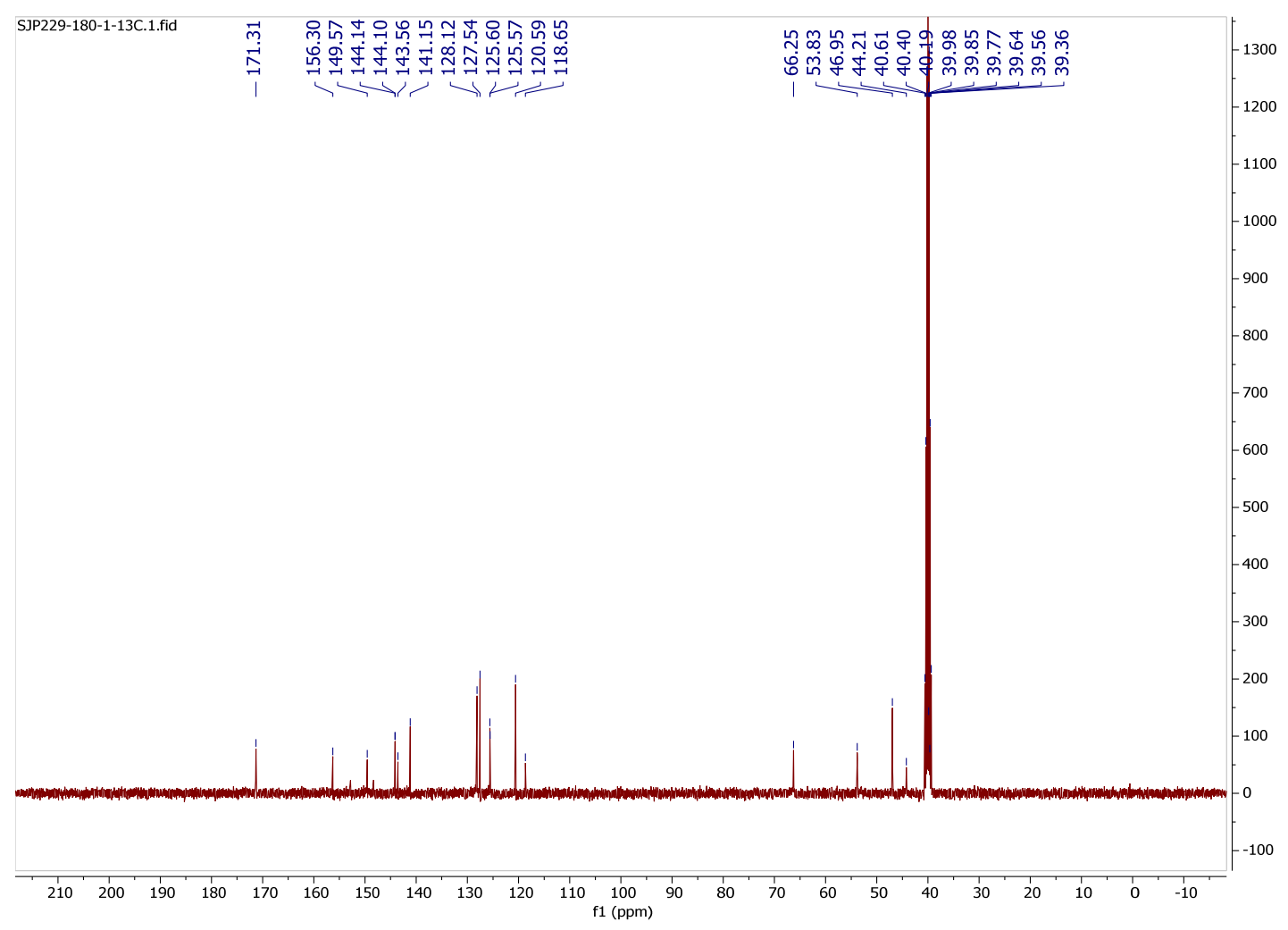




\section{${ }^{1} \mathrm{H}$ NMR (14a)}

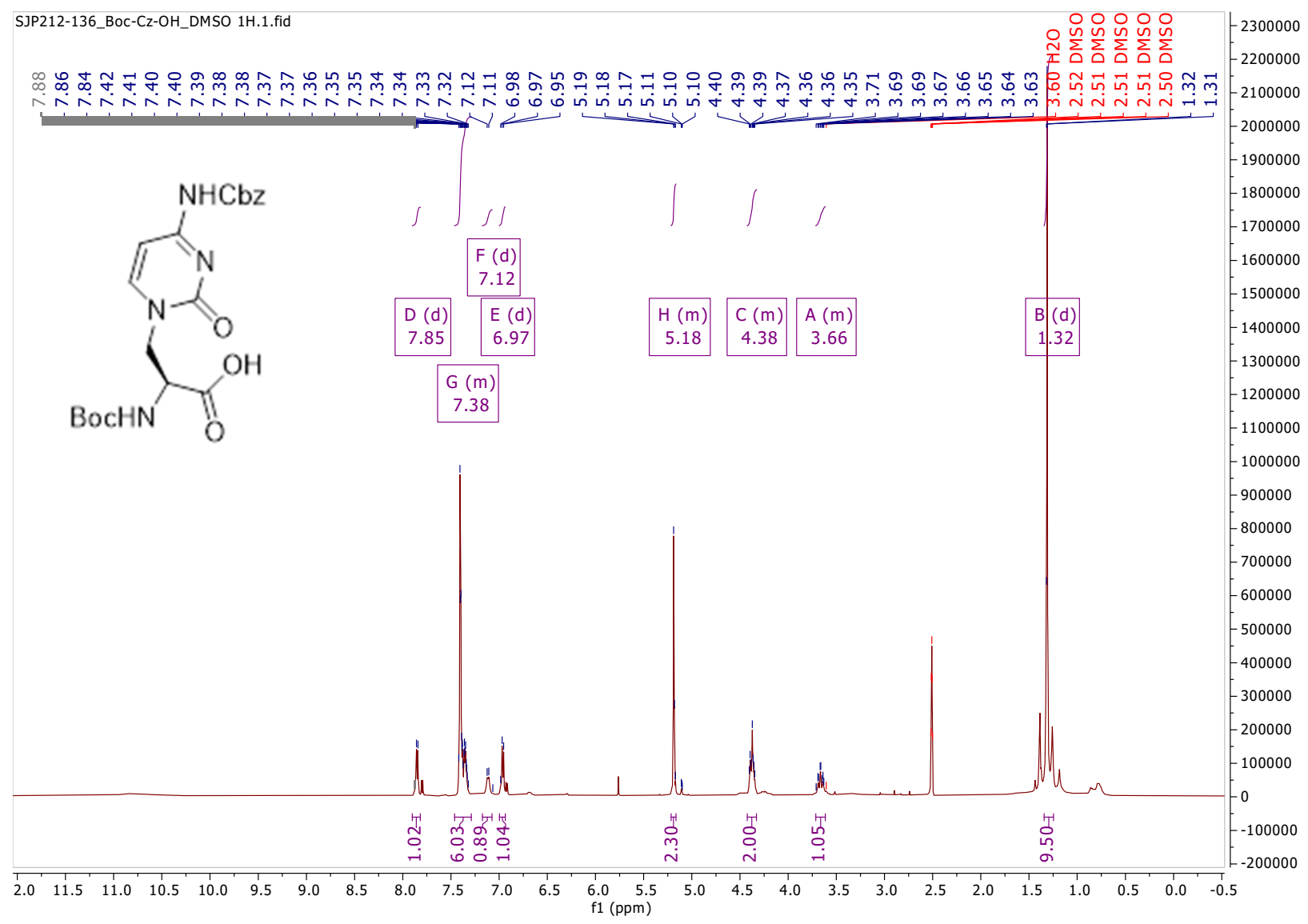




\section{${ }^{1} \mathrm{H}$ NMR (14)}

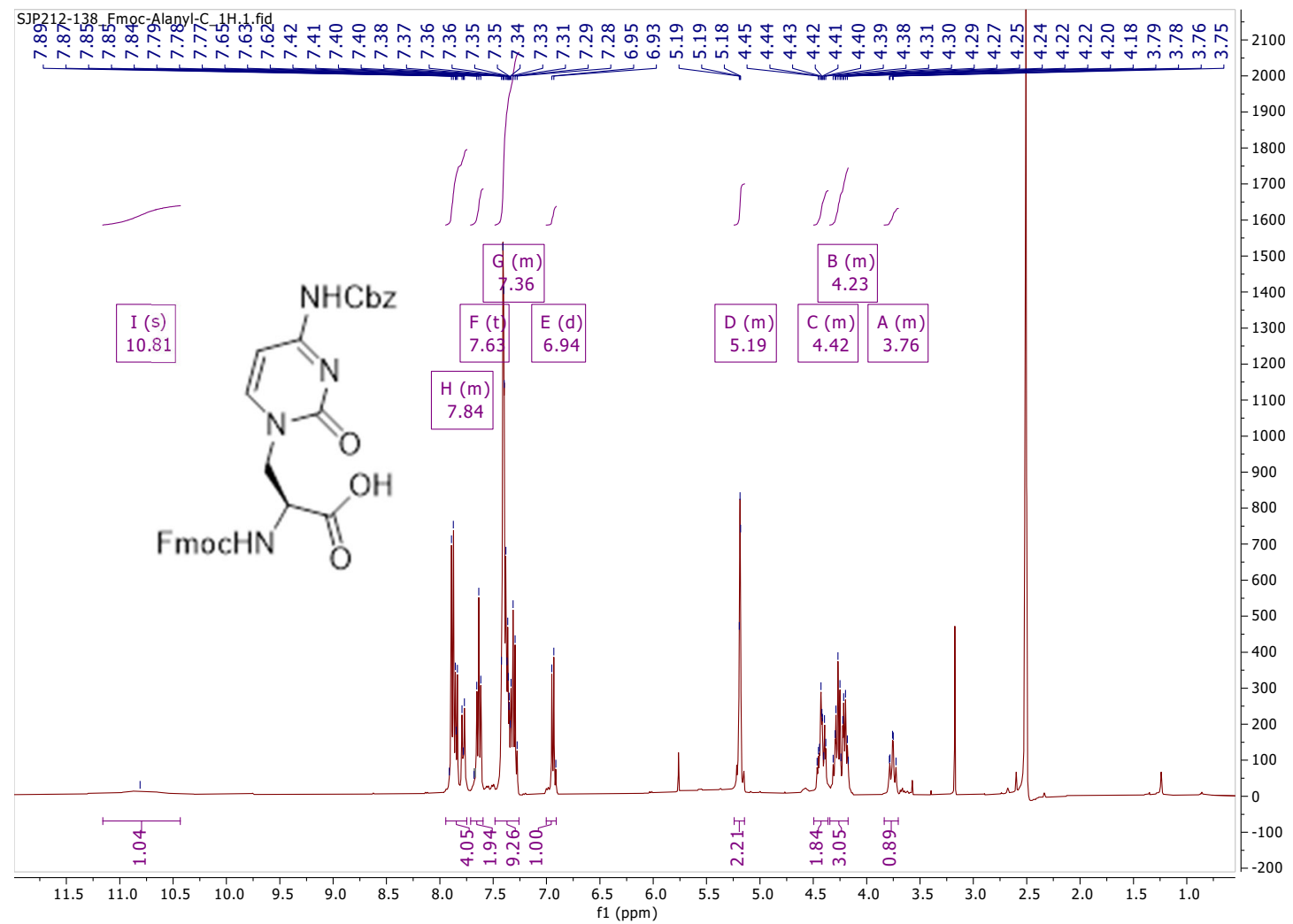

\section{${ }^{13}$ C NMR (14)}

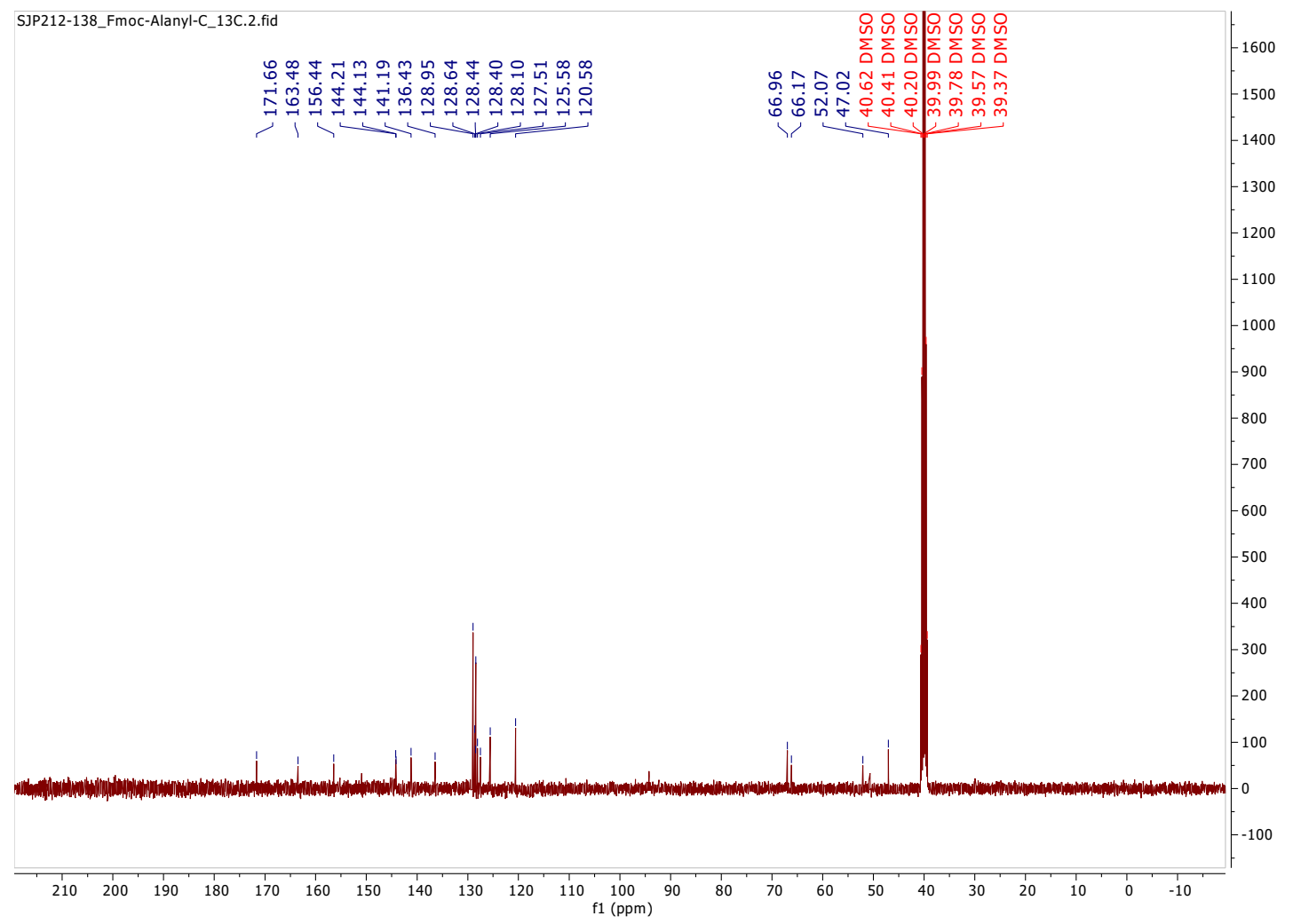




\section{${ }^{1} \mathrm{H}$ NMR (15a)}

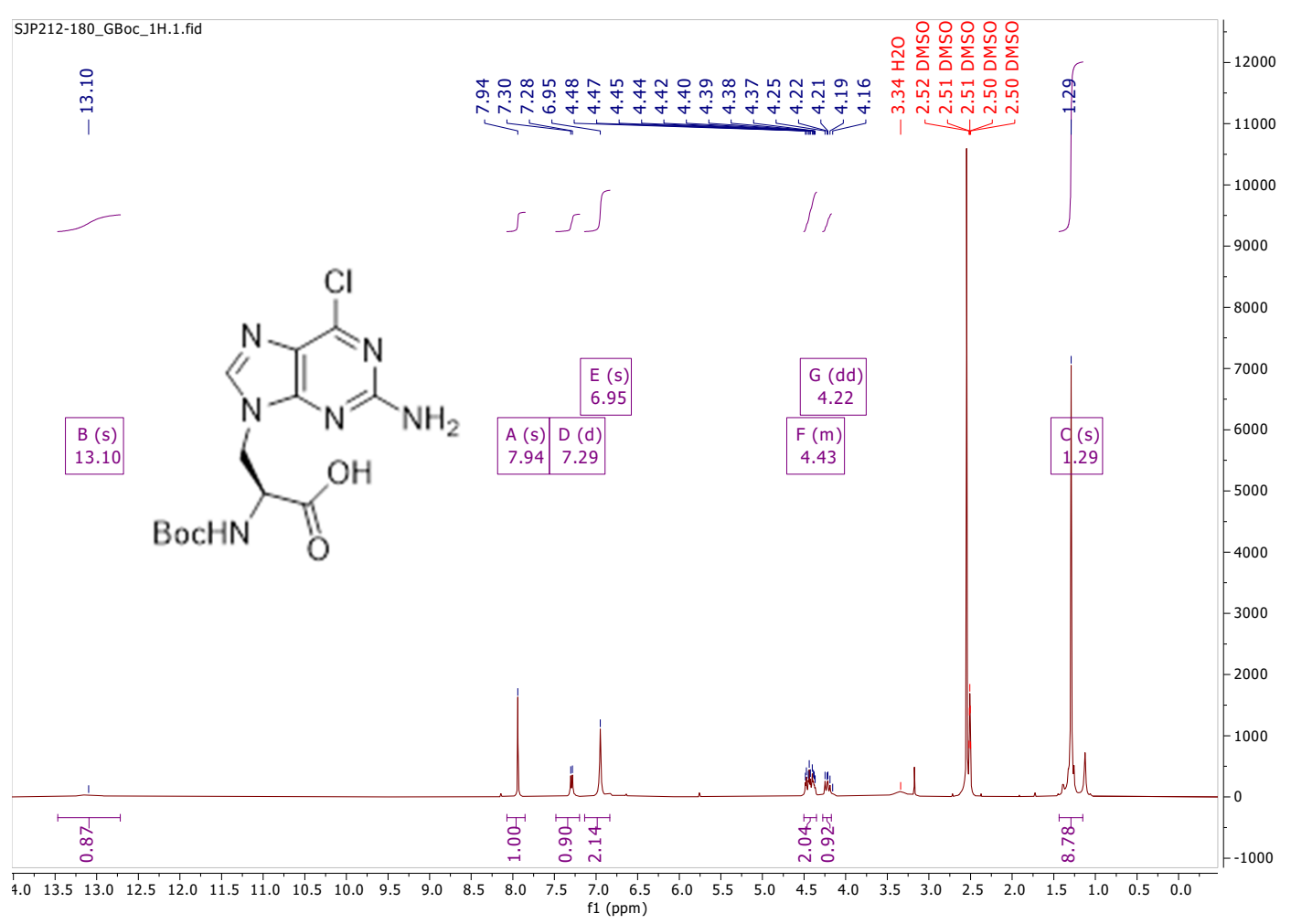

\section{${ }^{13}$ C NMR (15a)}

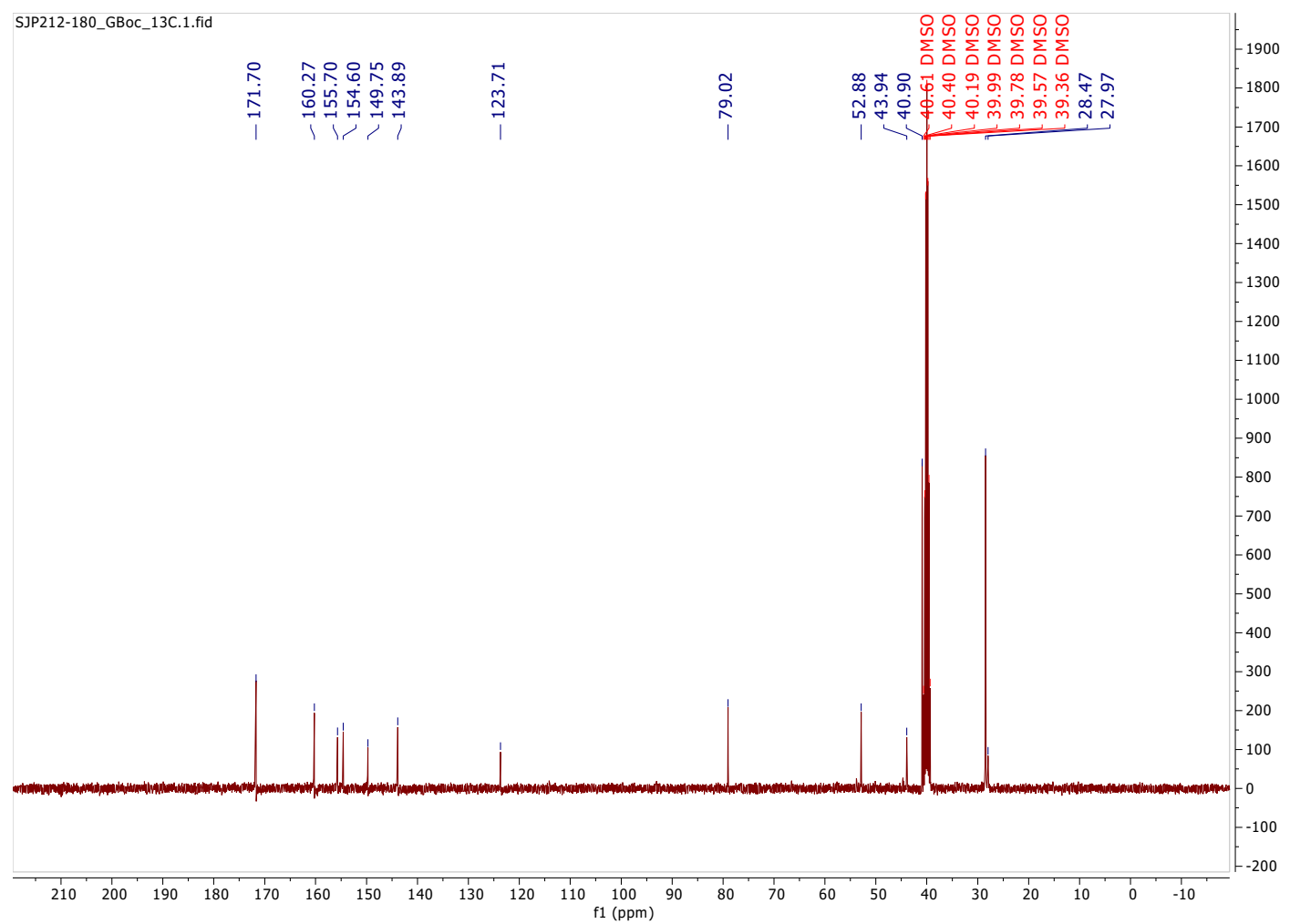




\section{${ }^{1} \mathrm{H}$ NMR (15)}

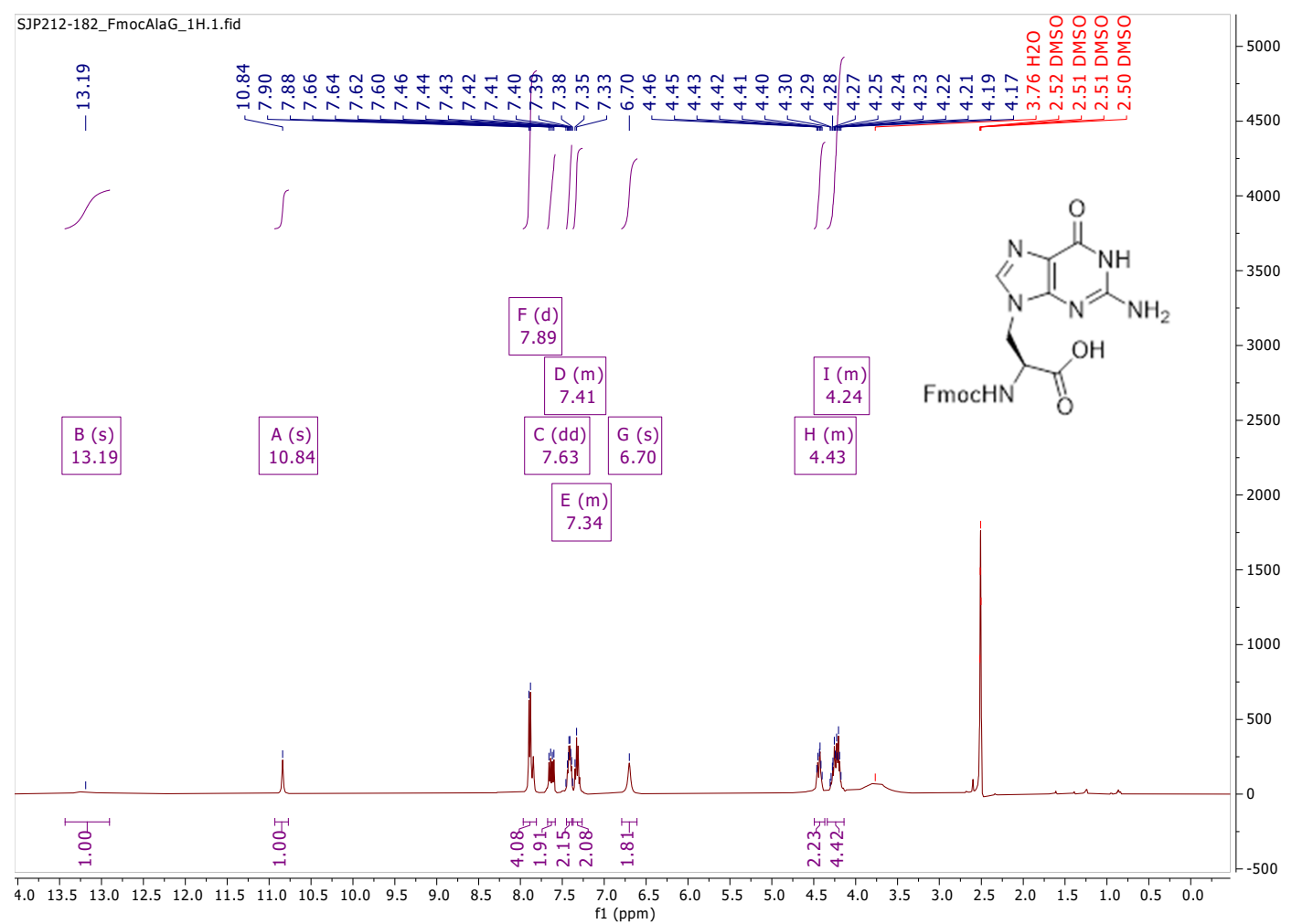

\section{${ }^{13}$ C NMR (15)}

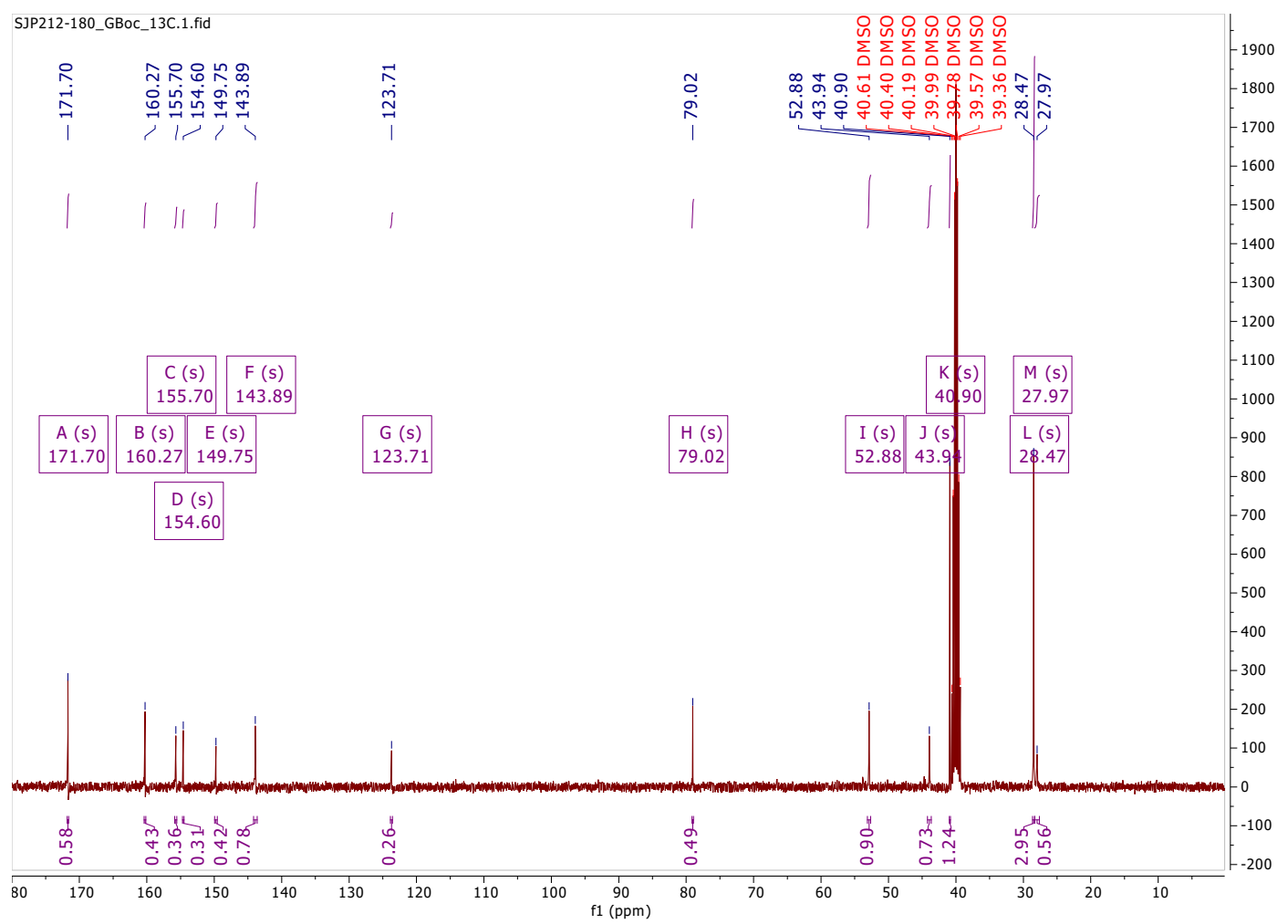




\section{${ }^{1} \mathrm{H}$ NMR (16a)}

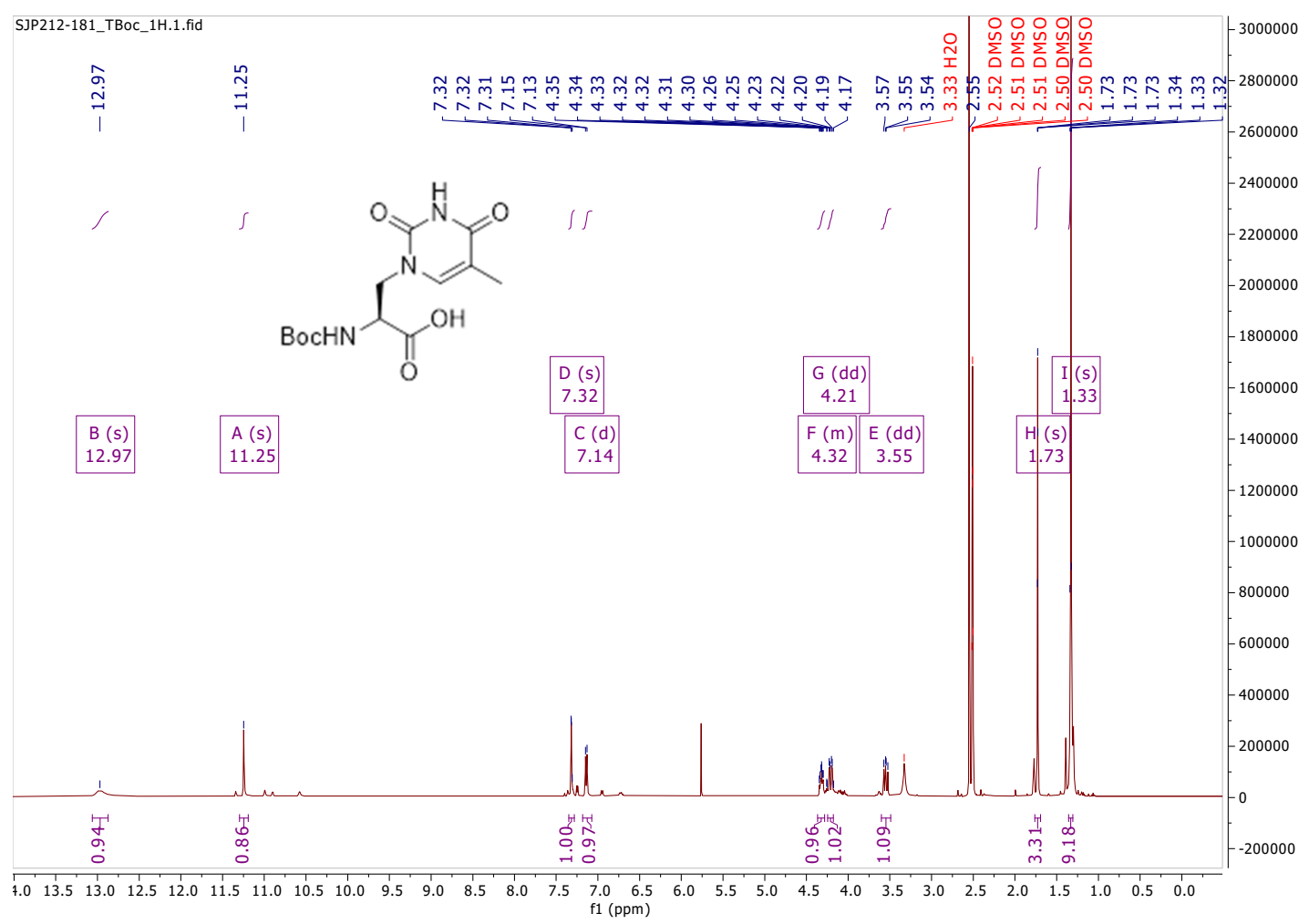

\section{${ }^{13}$ C NMR (16a)}

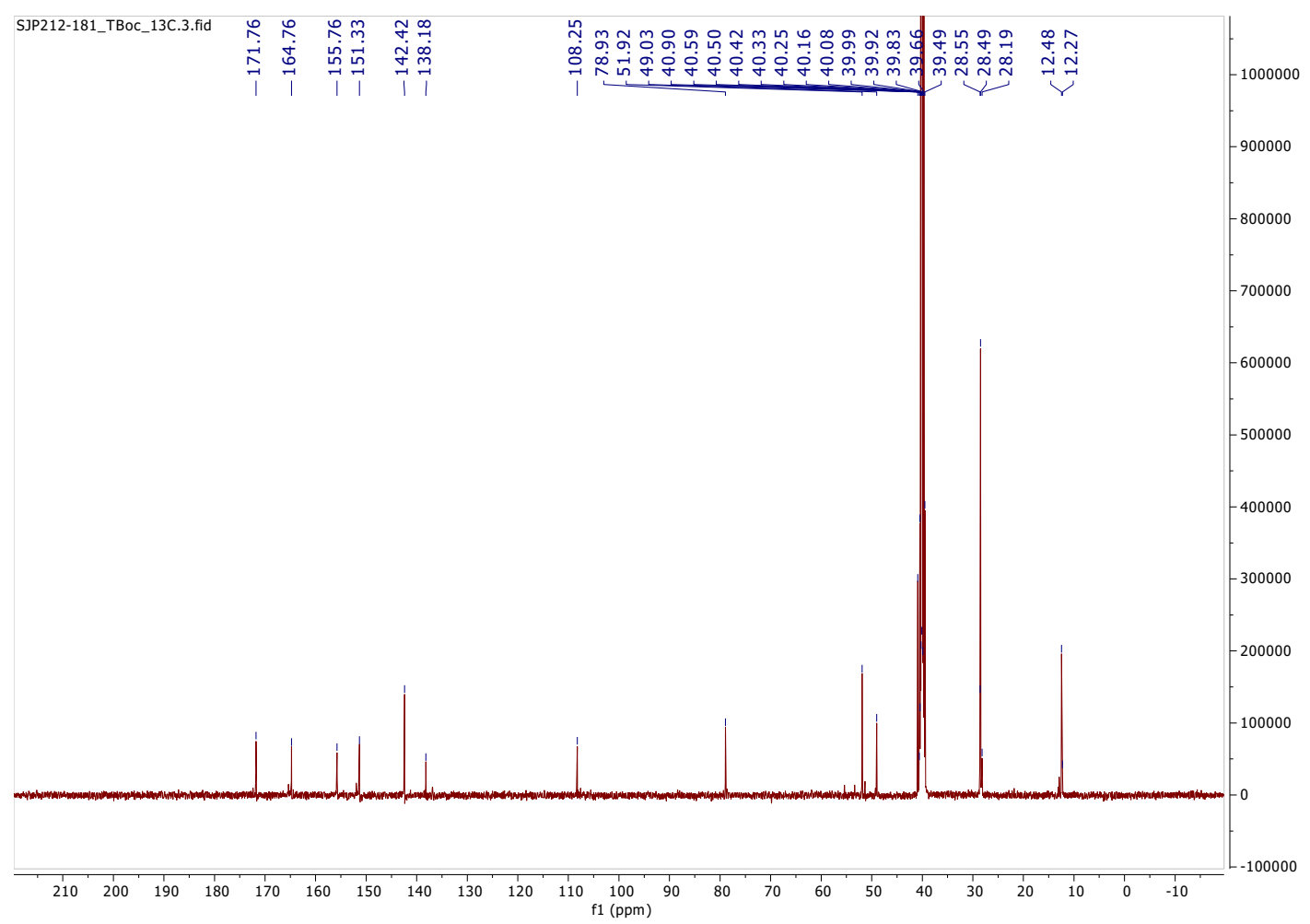




\section{${ }^{1} \mathrm{H}$ NMR (16)}

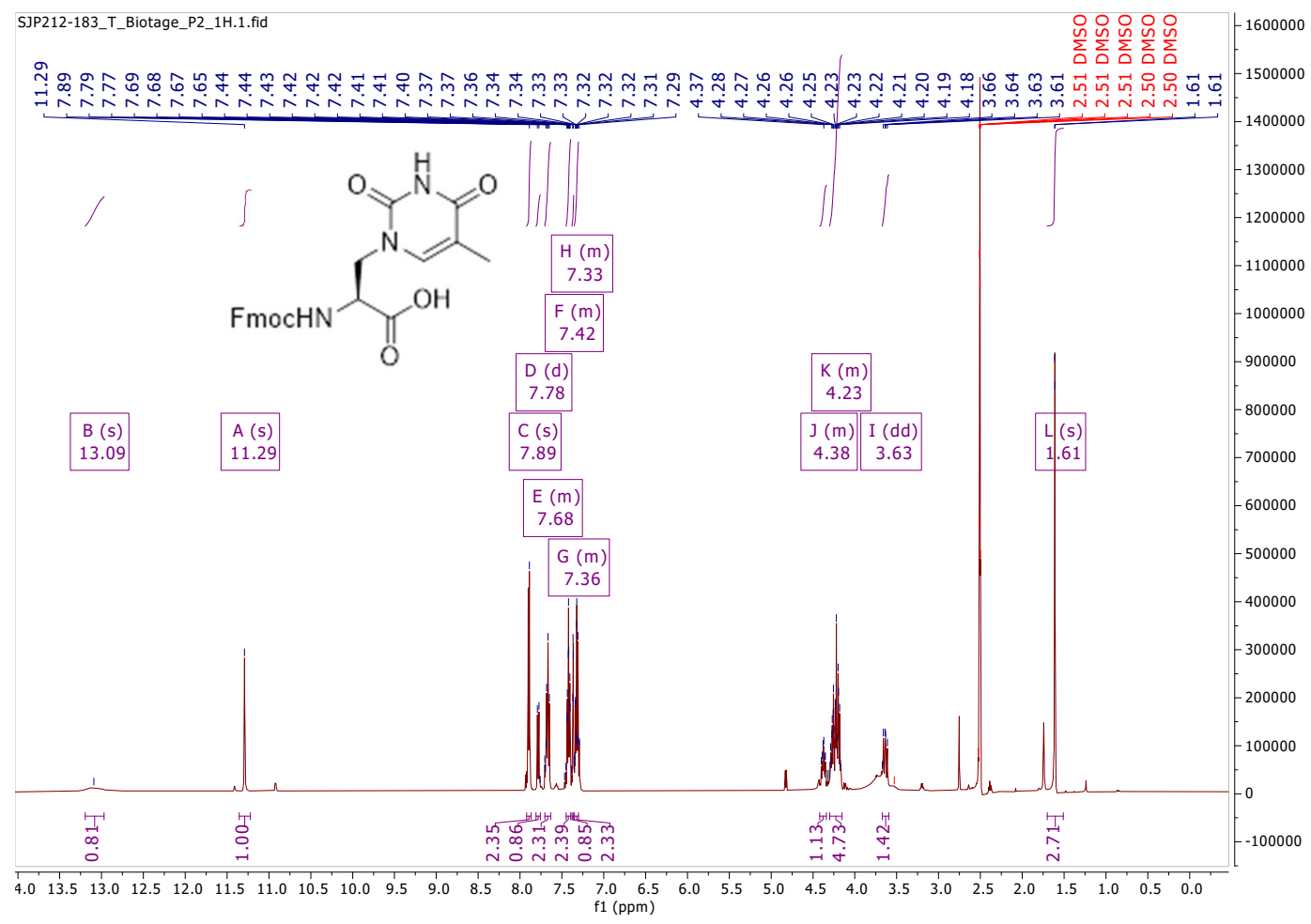

\section{${ }^{13}$ C NMR (16)}

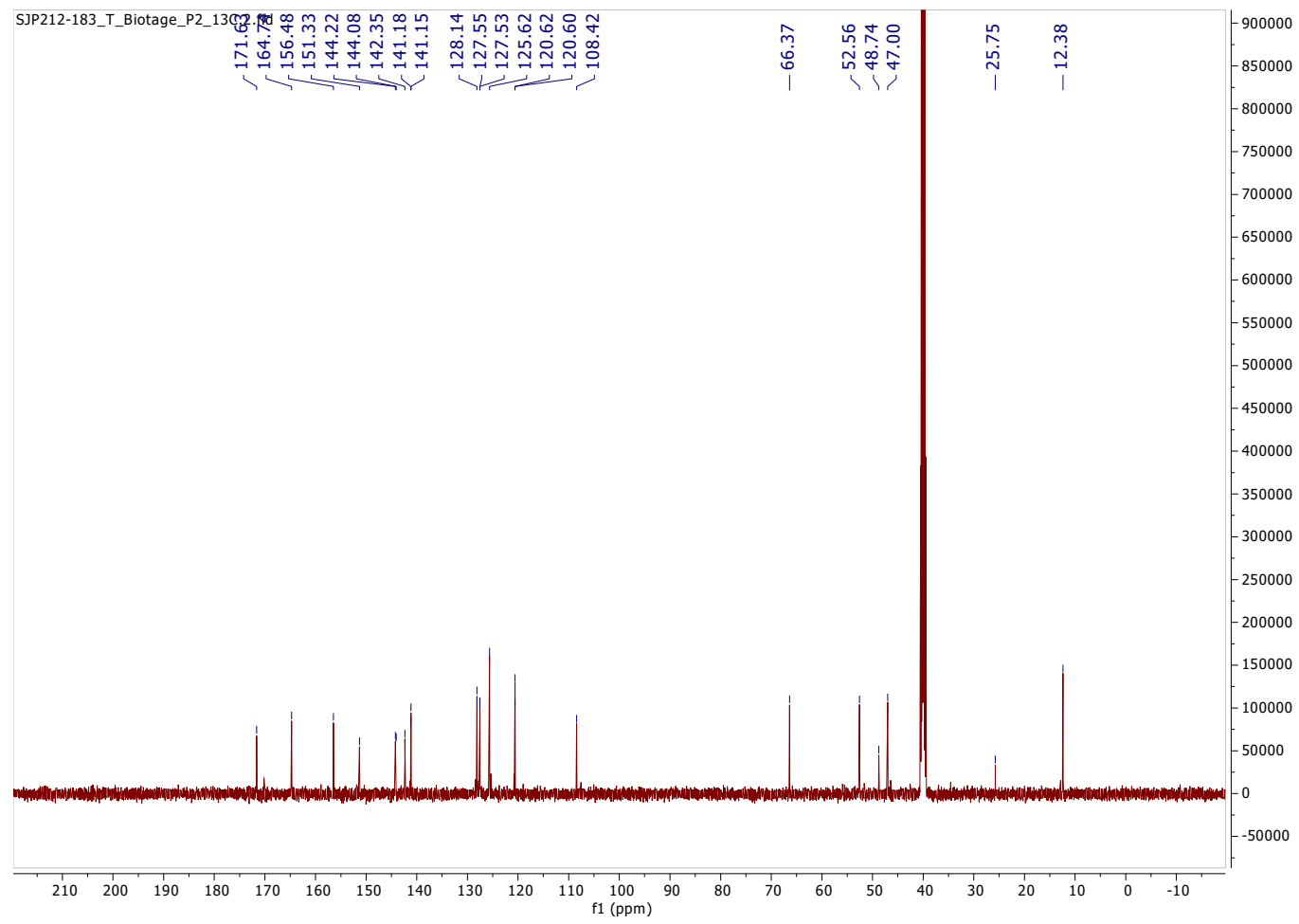




\section{${ }^{1} \mathrm{H}$ NMR (17a)}

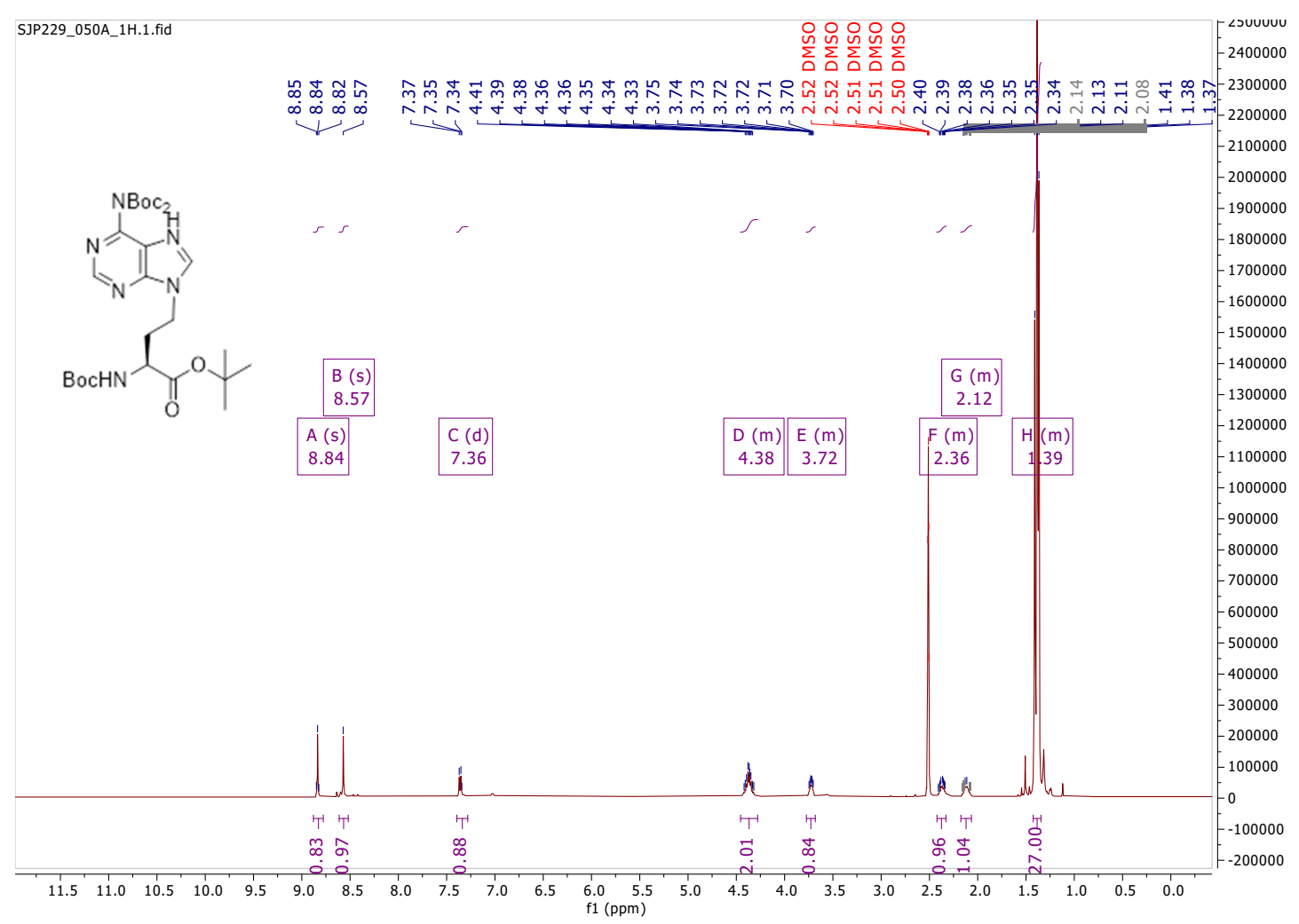

\section{${ }^{13}$ C NMR (17a)}

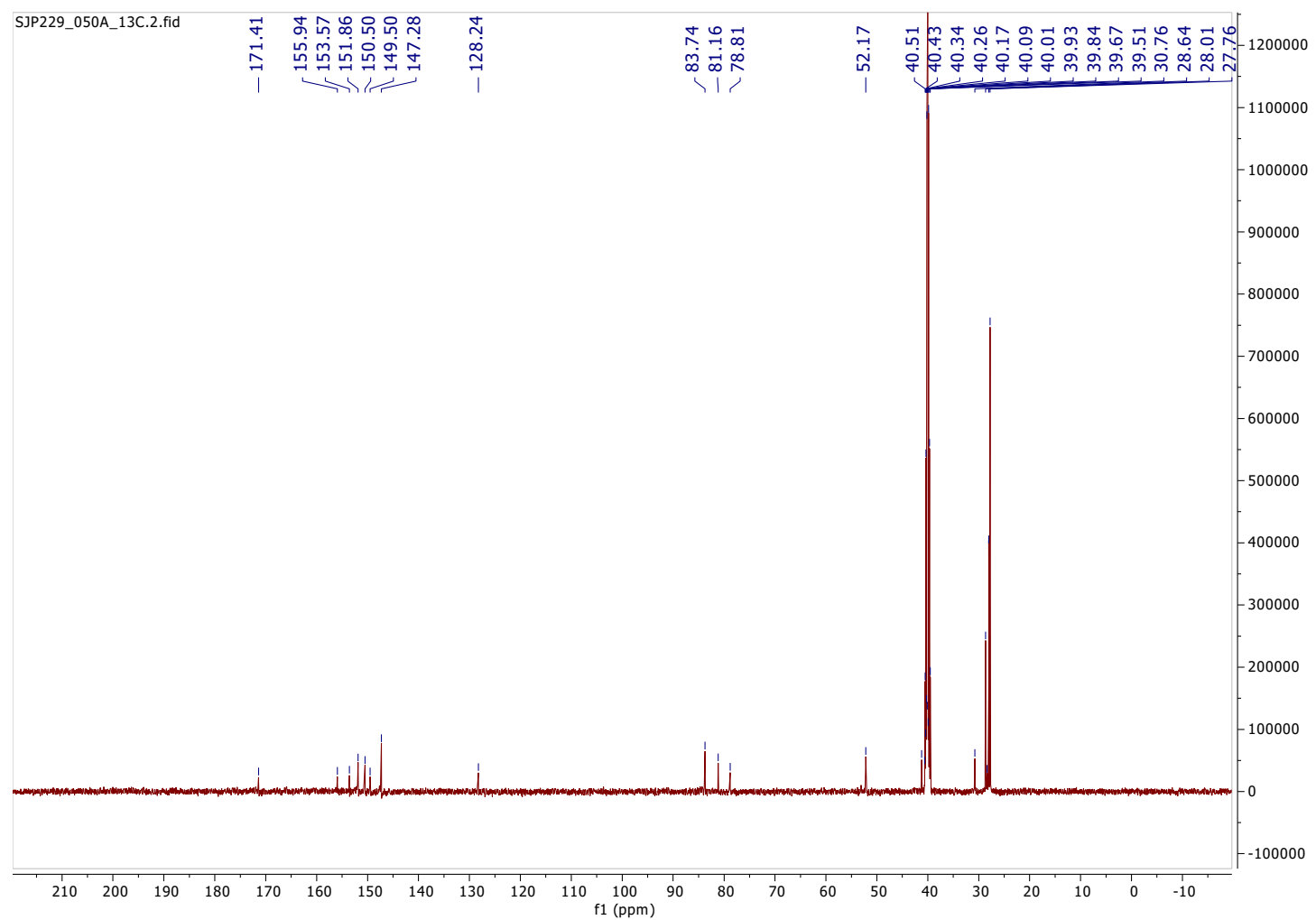




\section{${ }^{1} \mathrm{H}$ NMR (17)}

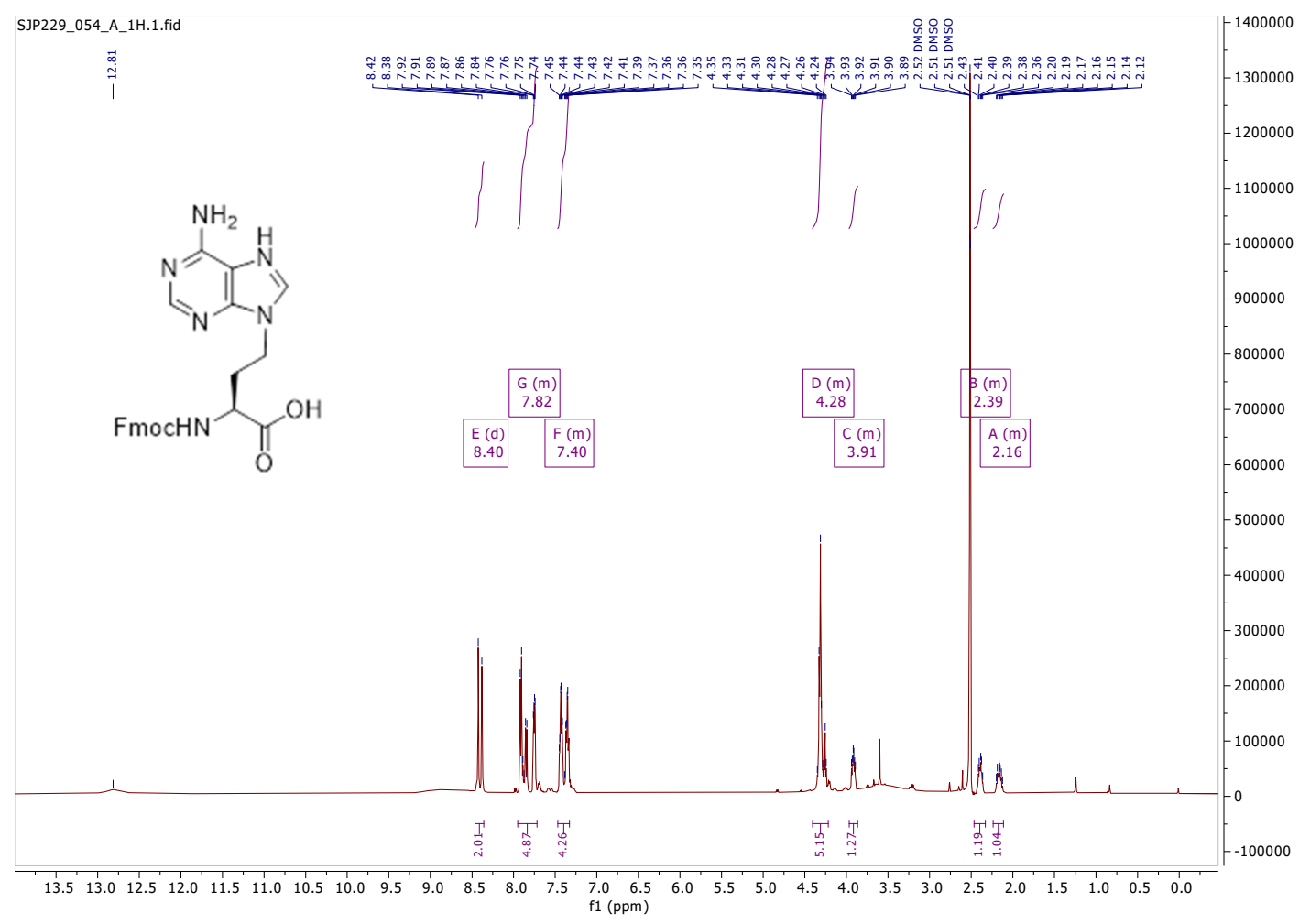

\section{${ }^{13}$ C NMR (17)}

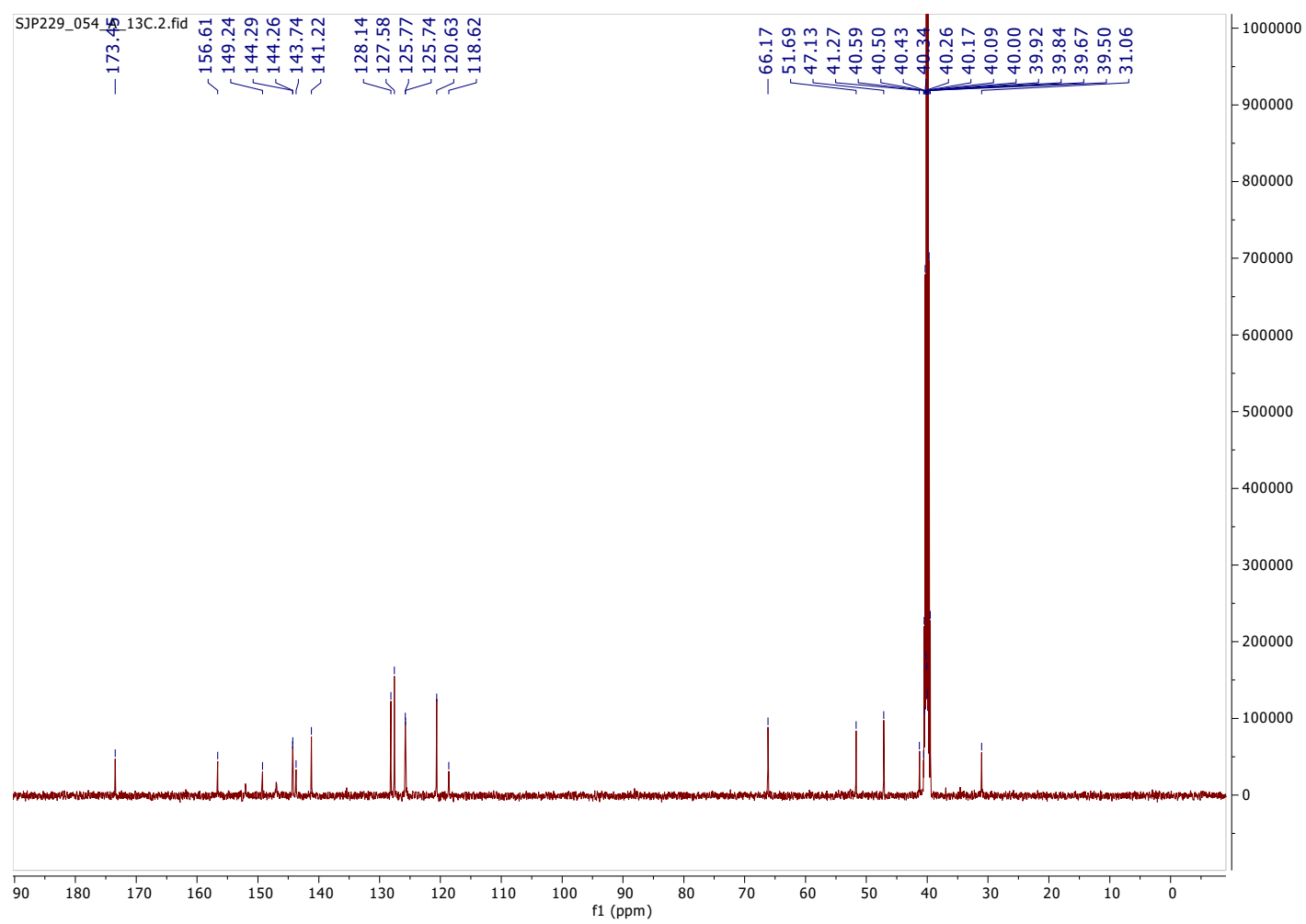




\section{${ }^{1} \mathrm{H}$ NMR (18a)}

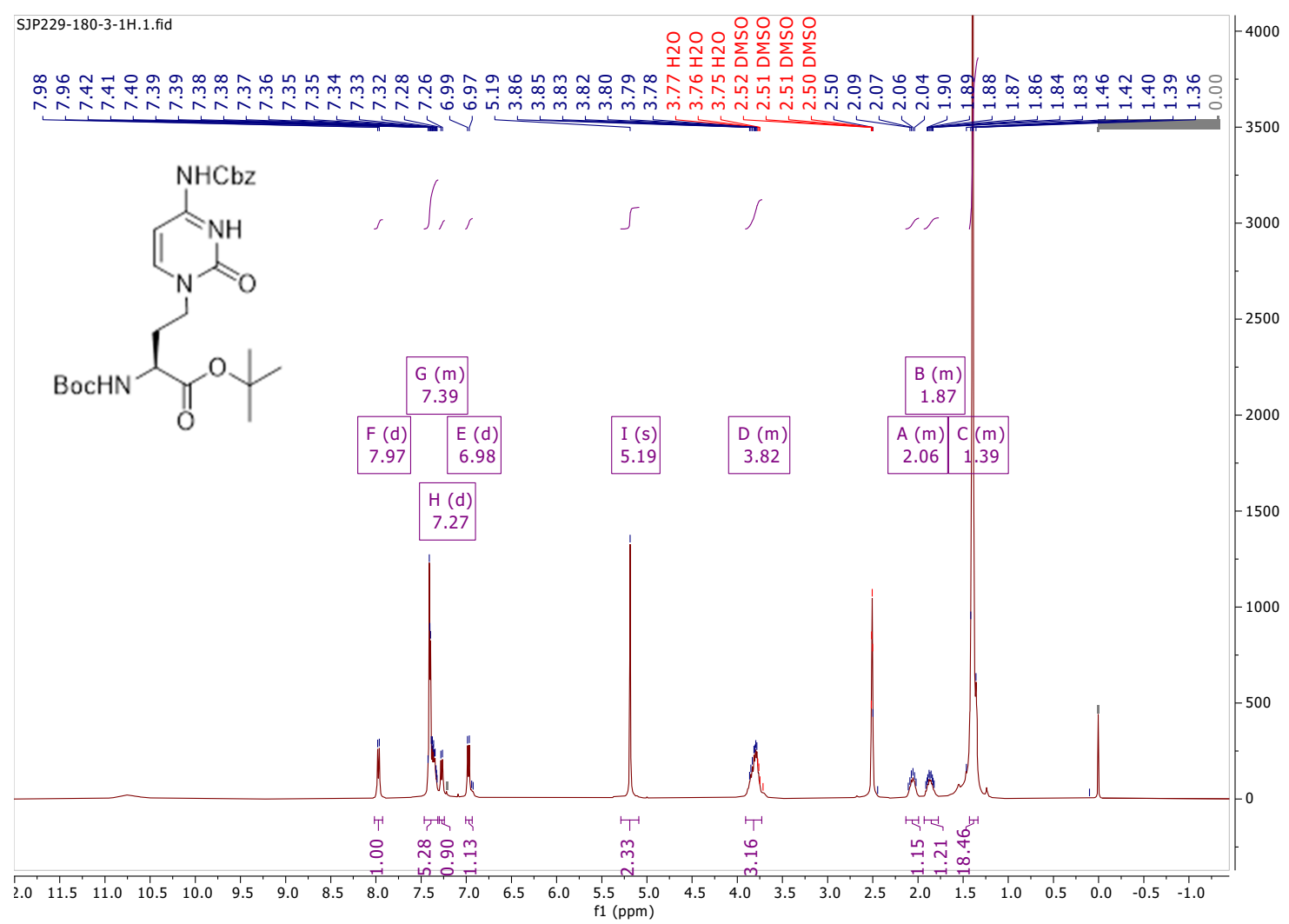

\section{${ }^{13} \mathrm{C}$ NMR (18a)}

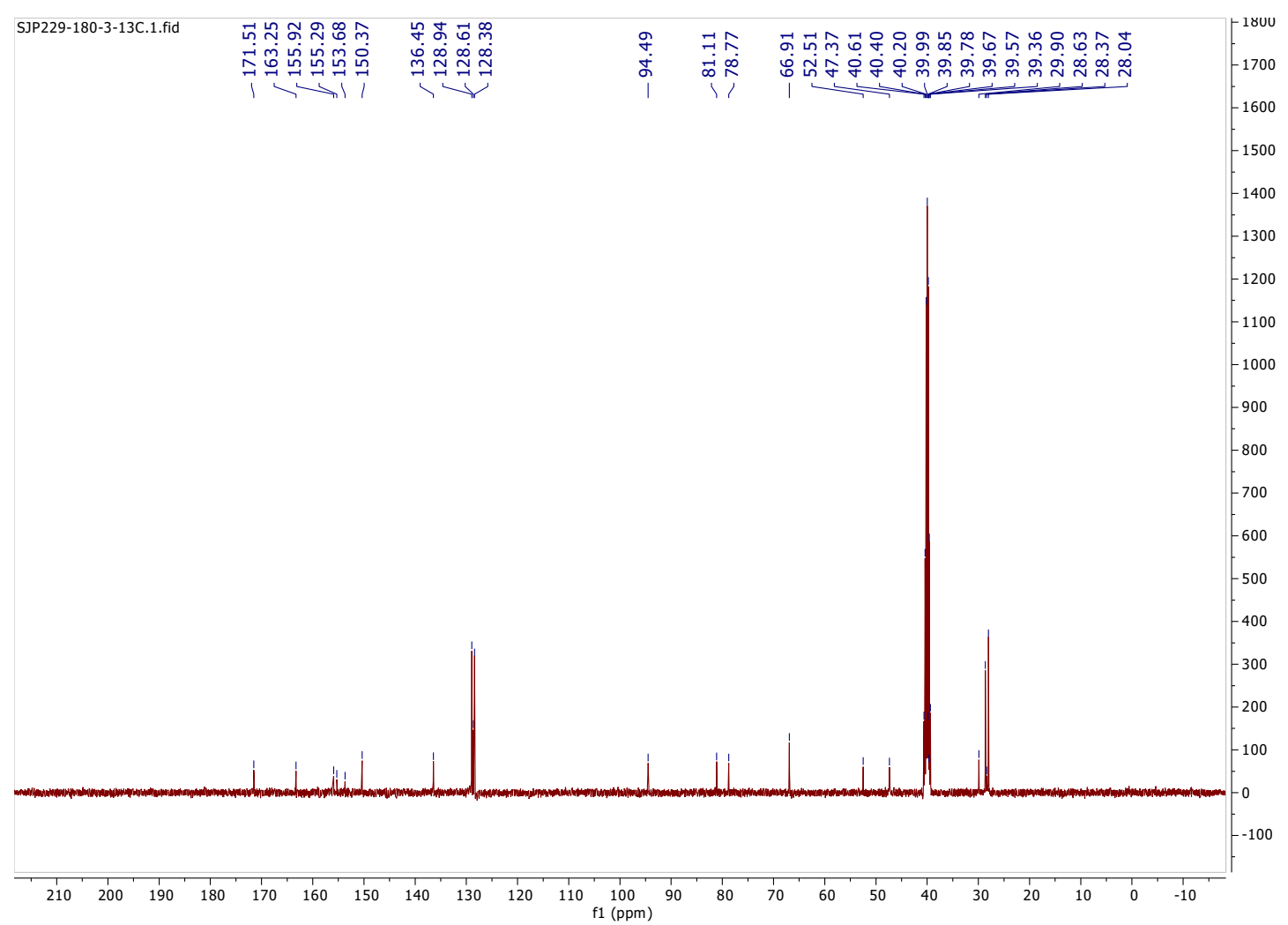




\section{${ }^{1} \mathrm{H}$ NMR (18)}

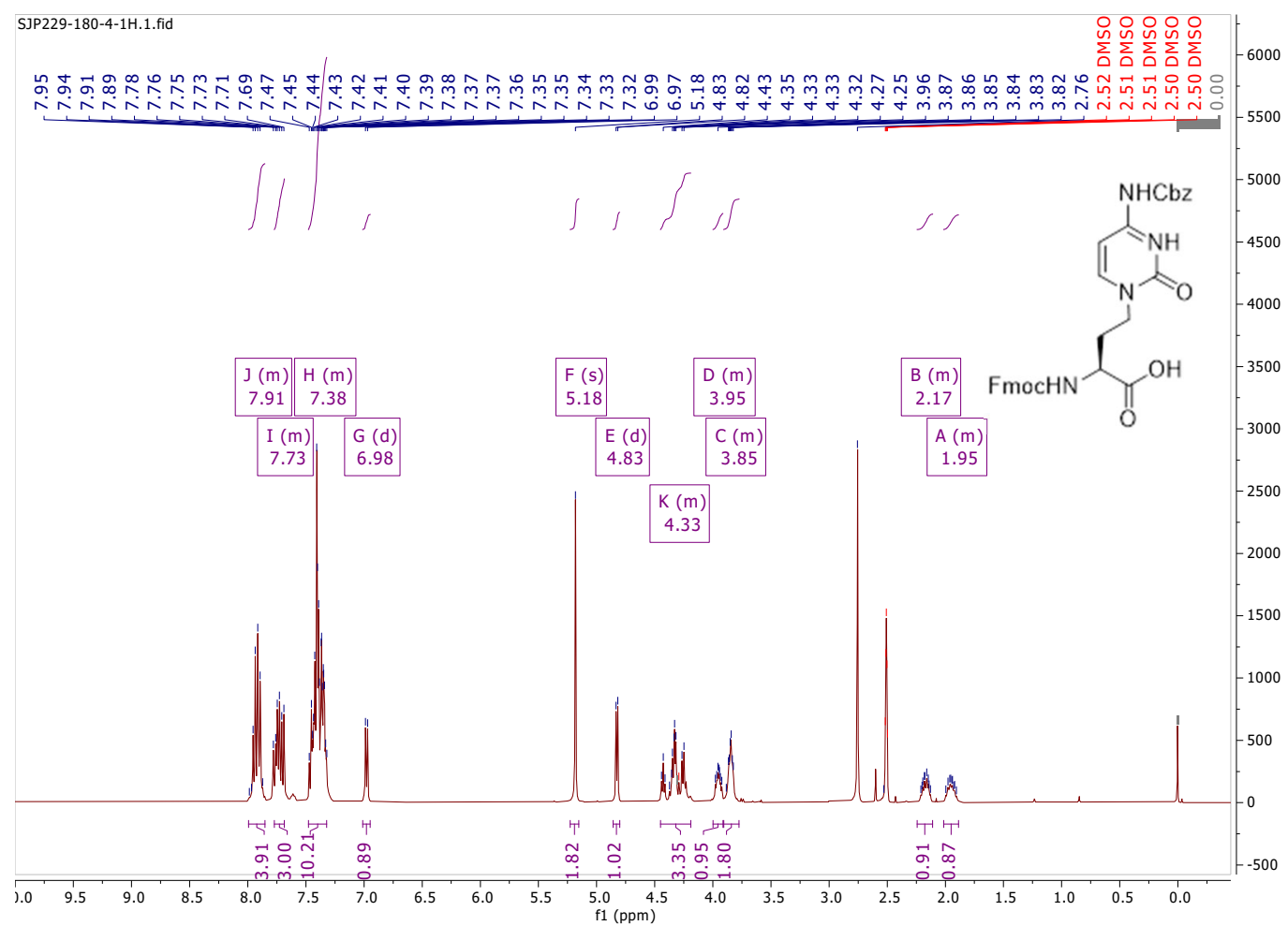

${ }^{13}$ C NMR (18)

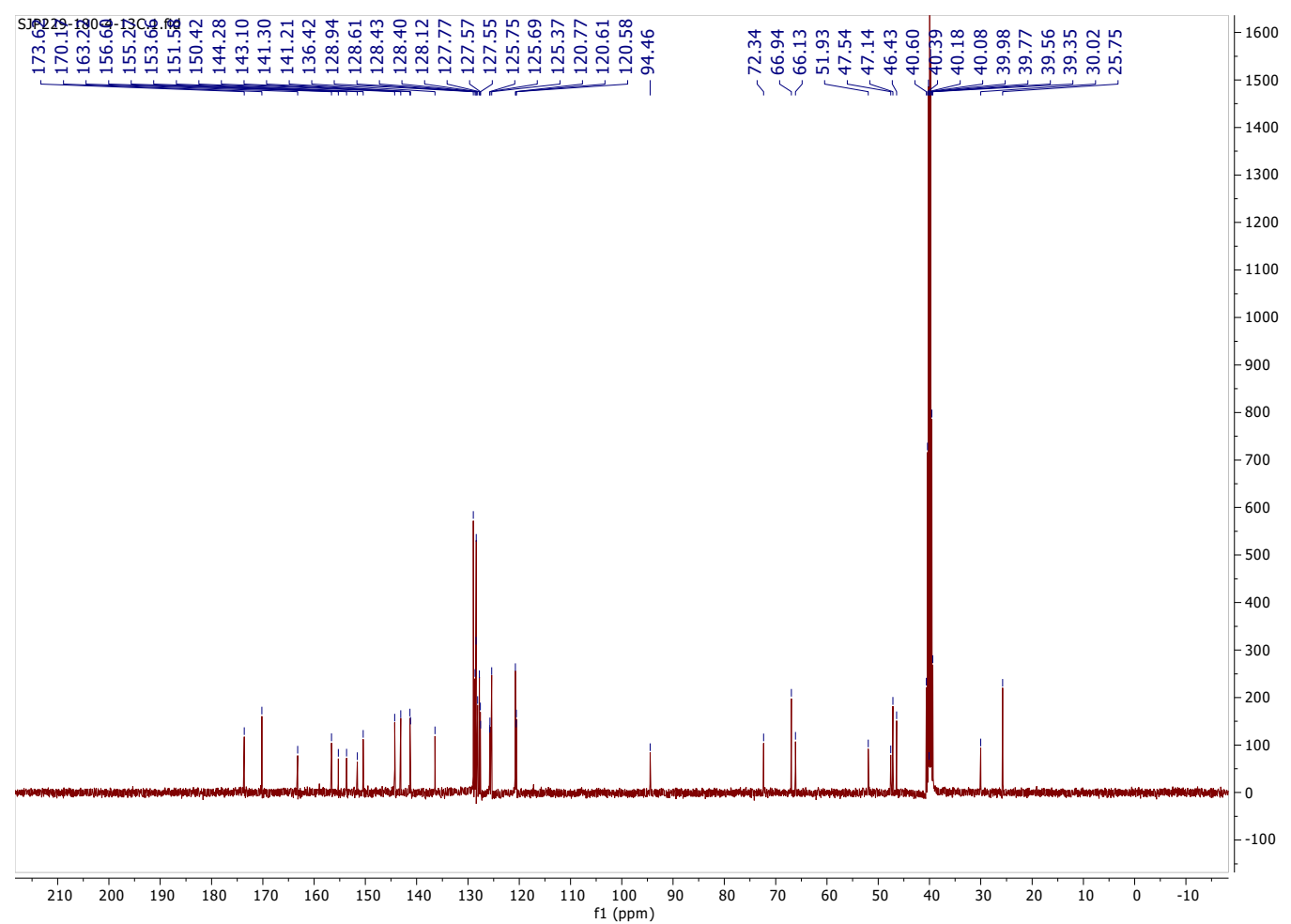




\section{${ }^{1}$ H NMR (19a)}

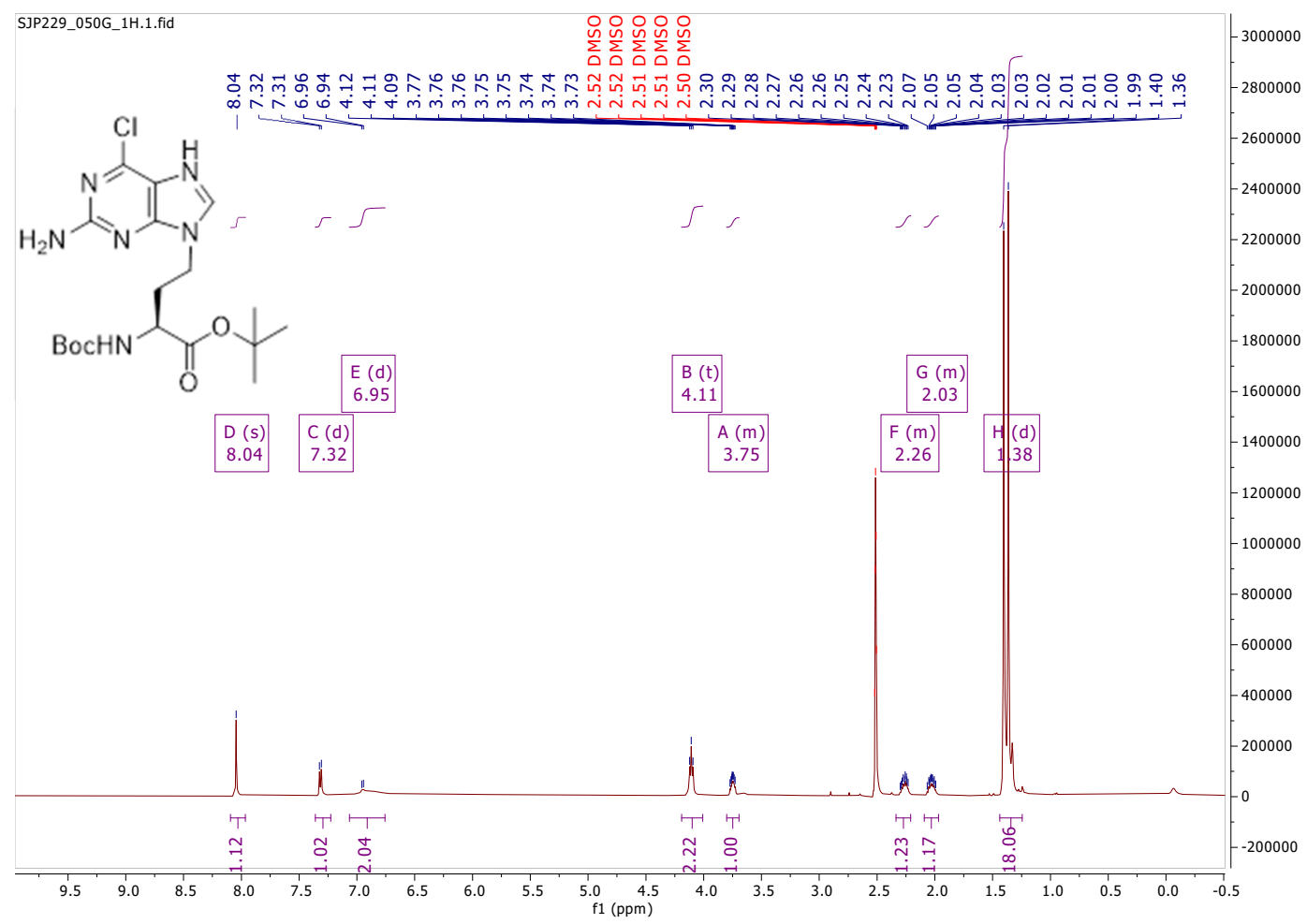

\section{${ }^{13}$ C NMR (19a)}

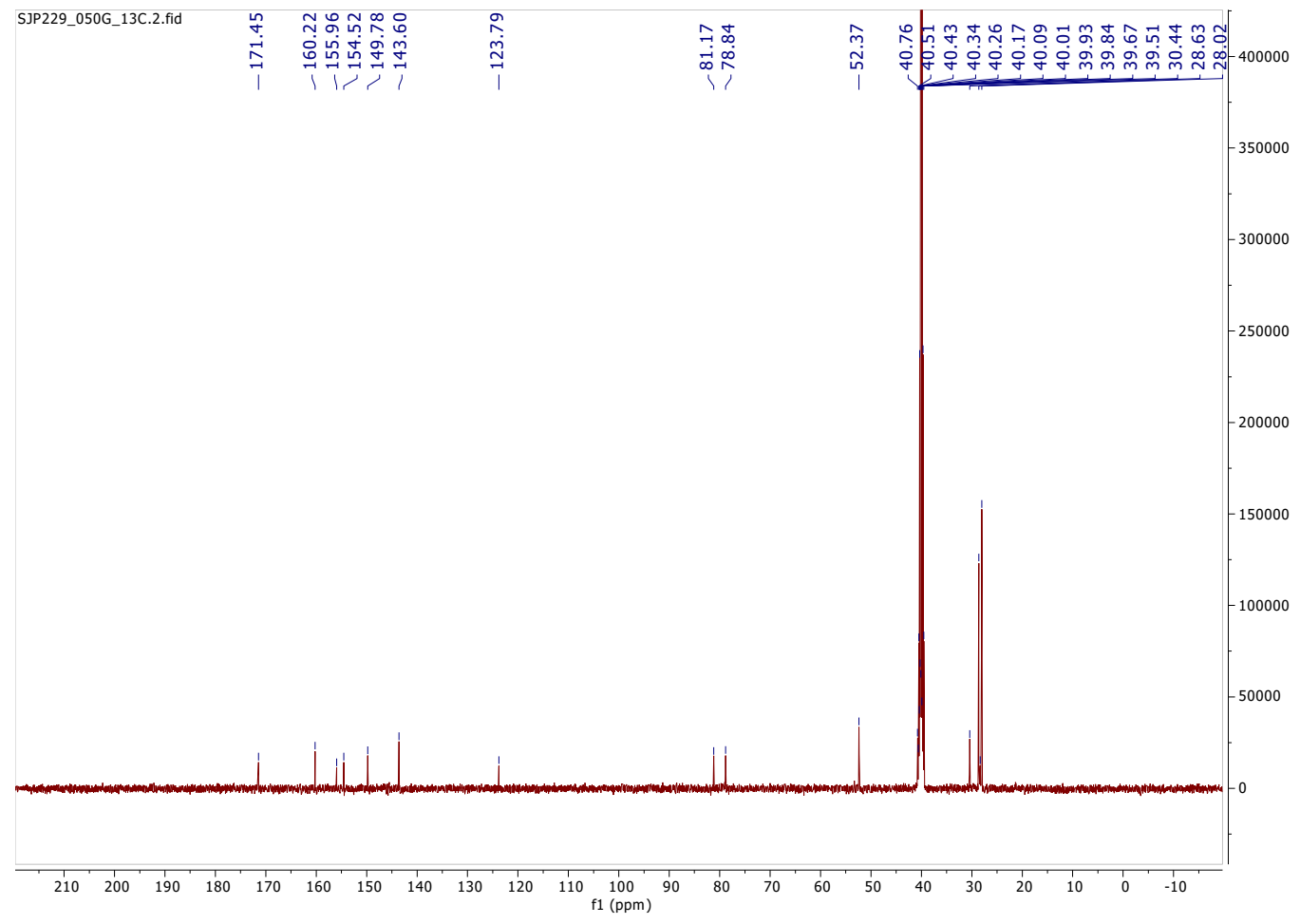




\section{${ }^{1} \mathrm{H}$ NMR (19)}

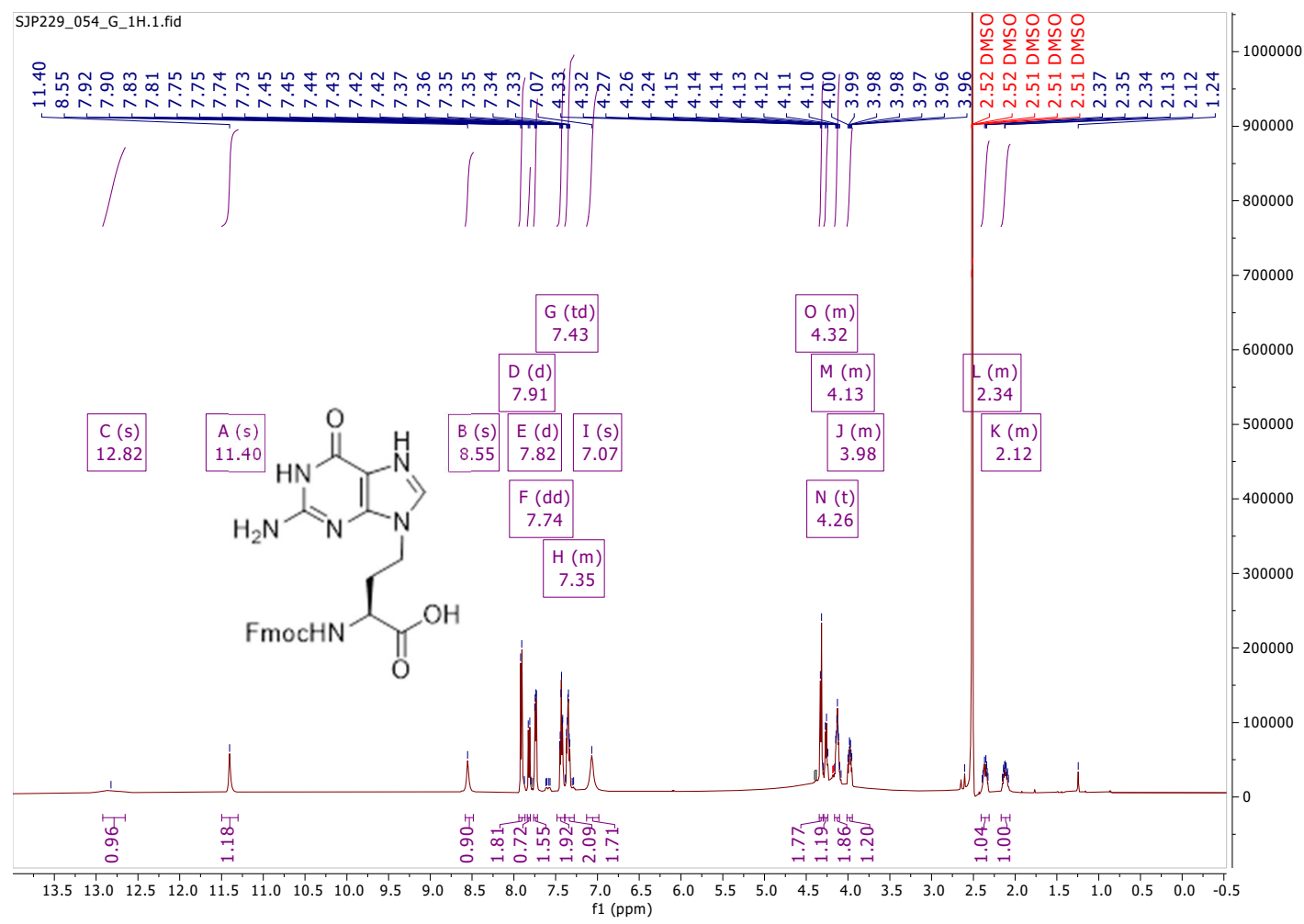

\section{${ }^{13}$ C NMR (19)}

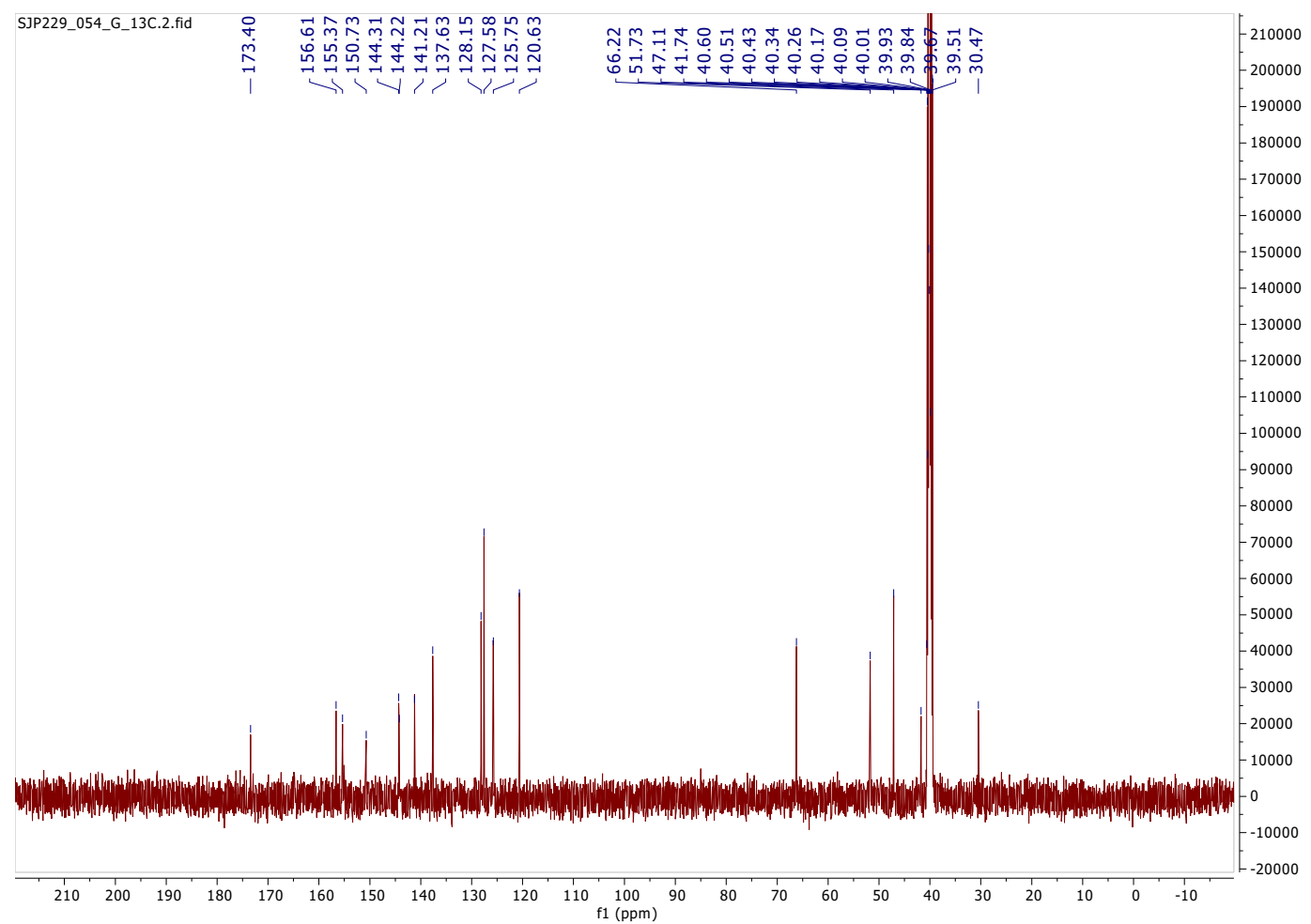




\section{${ }^{1} \mathrm{H}$ NMR (20)}

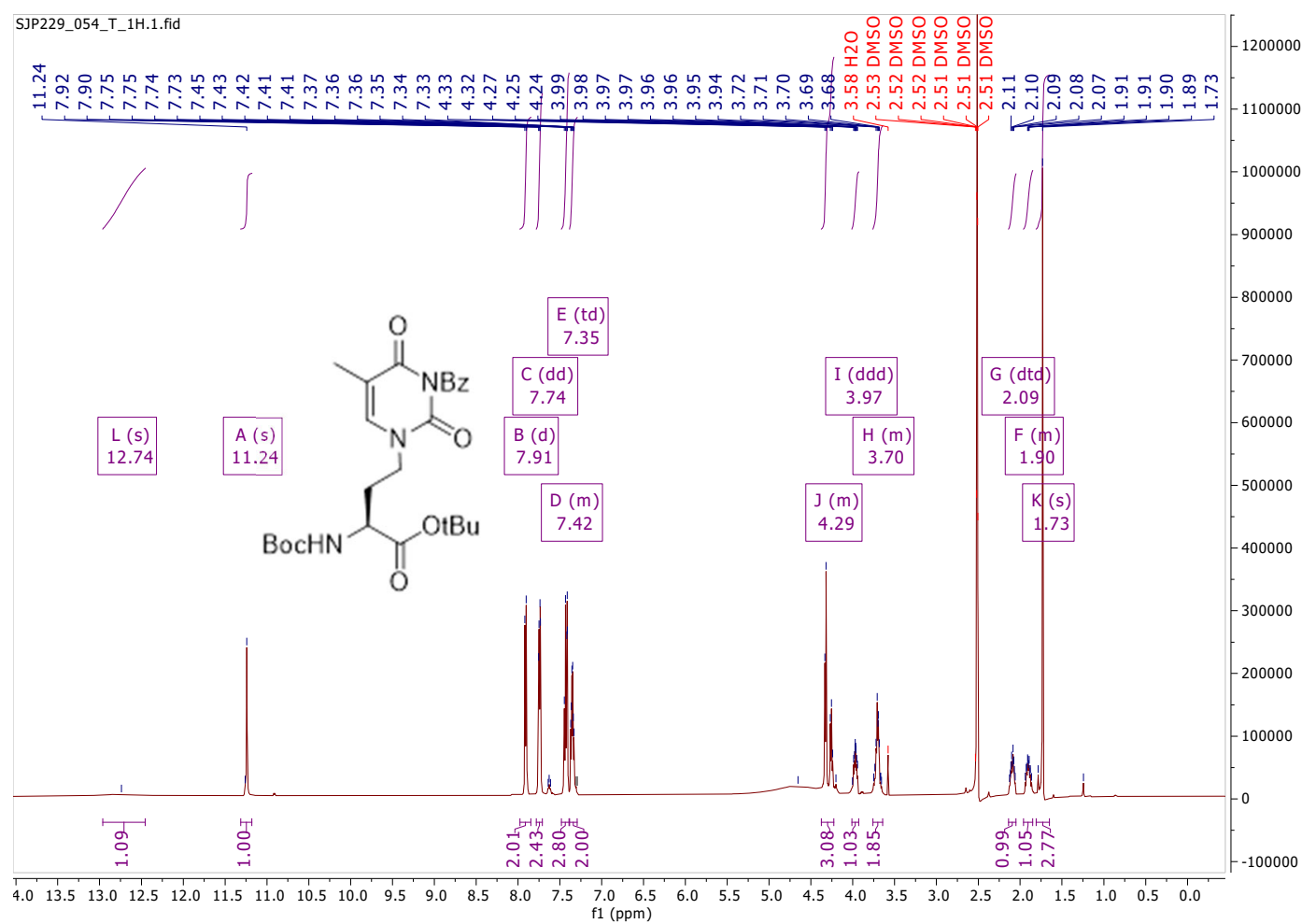

\section{${ }^{13}$ C NMR (20)}

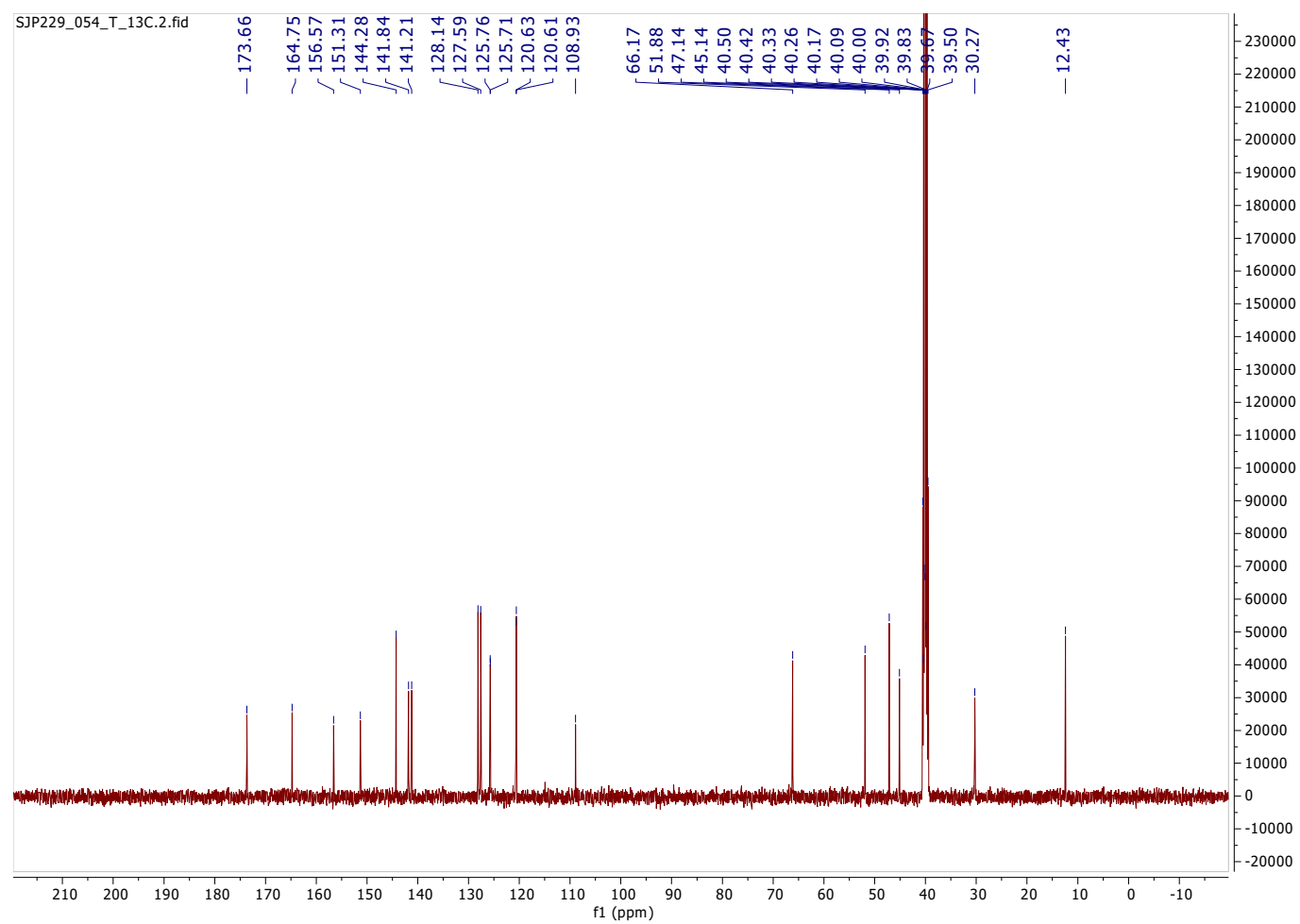




\section{${ }^{1} \mathrm{H}$ NMR (21)}

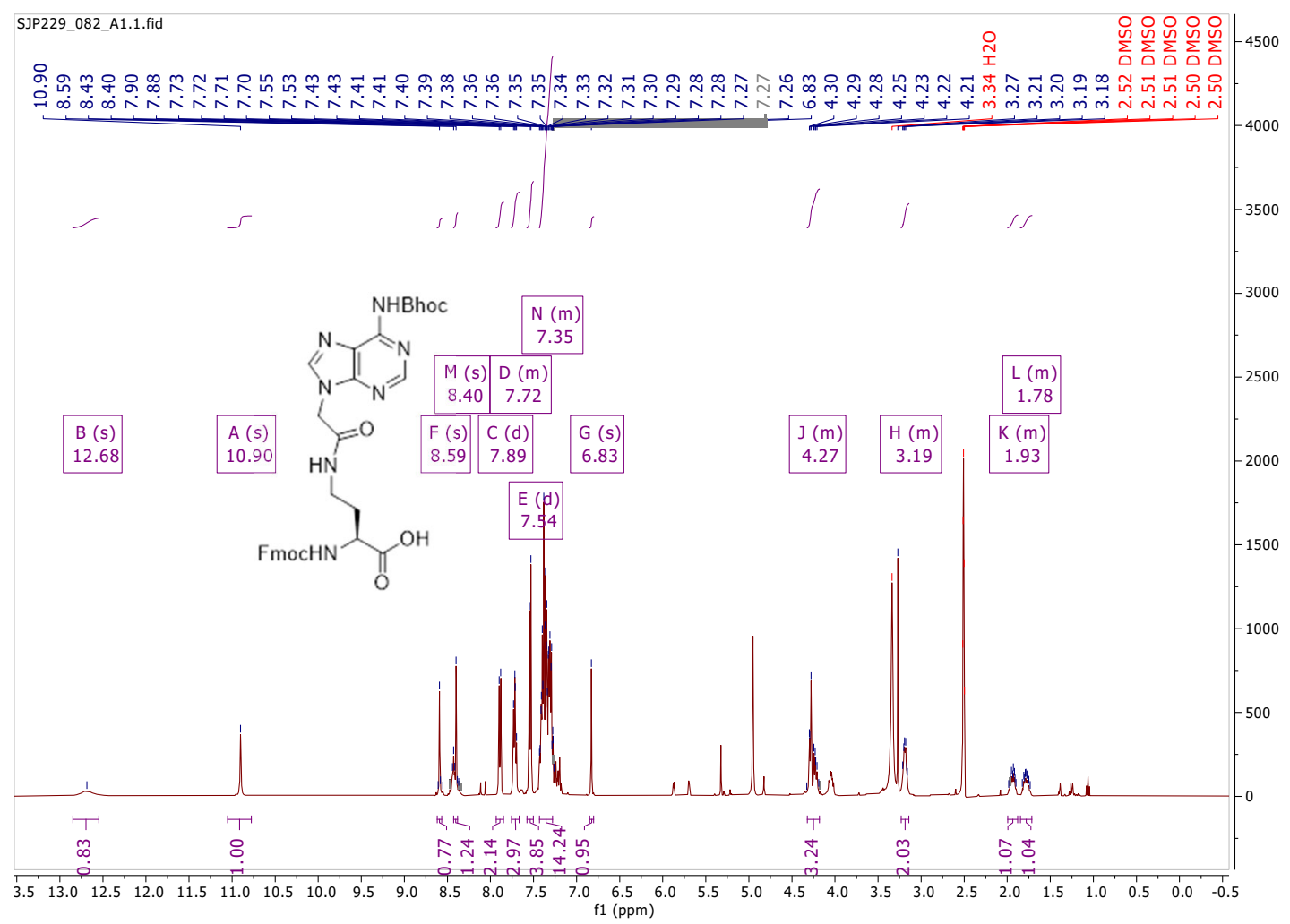

\section{${ }^{13}$ C NMR (21)}

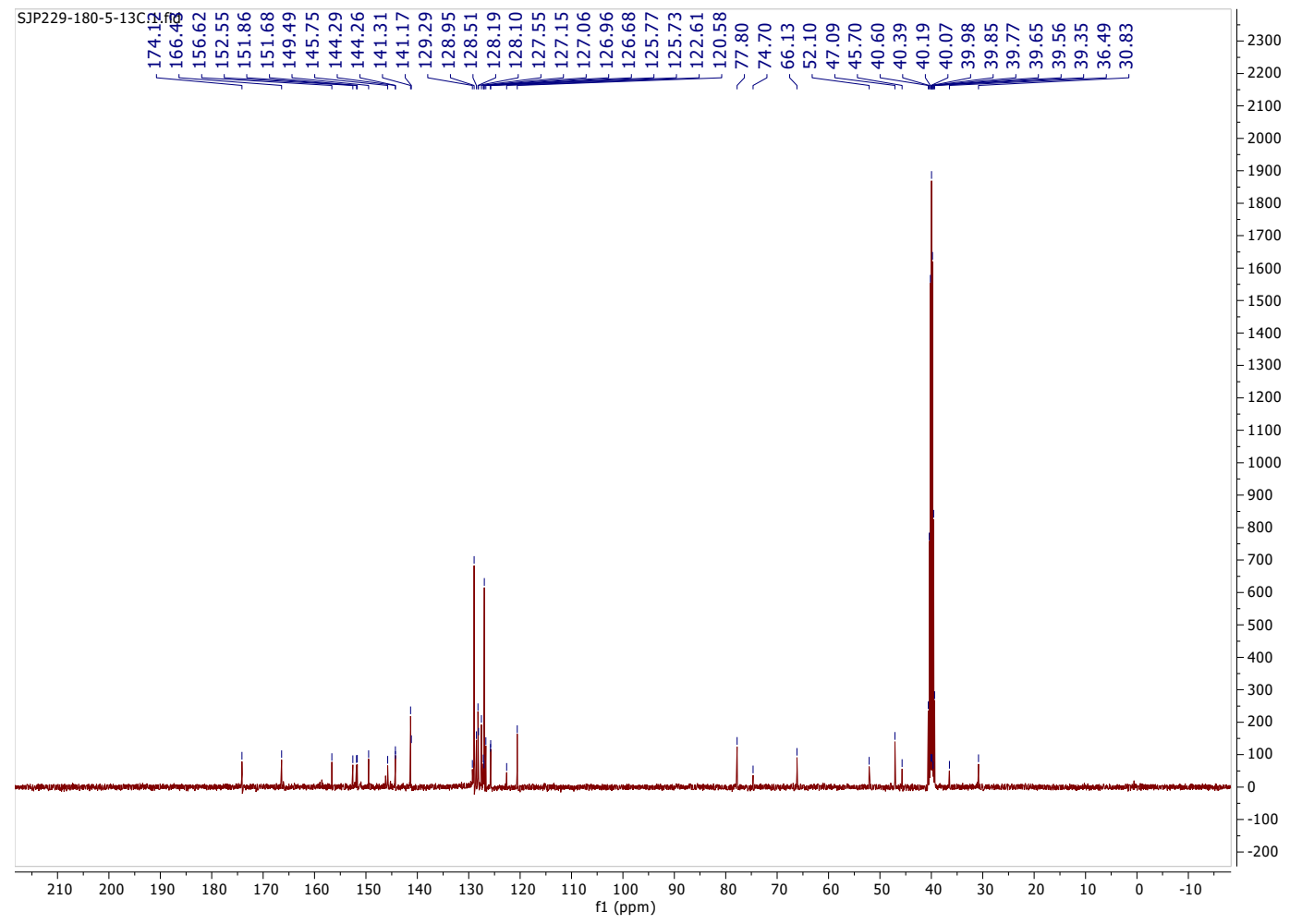




\section{${ }^{1}$ H NMR (22)}

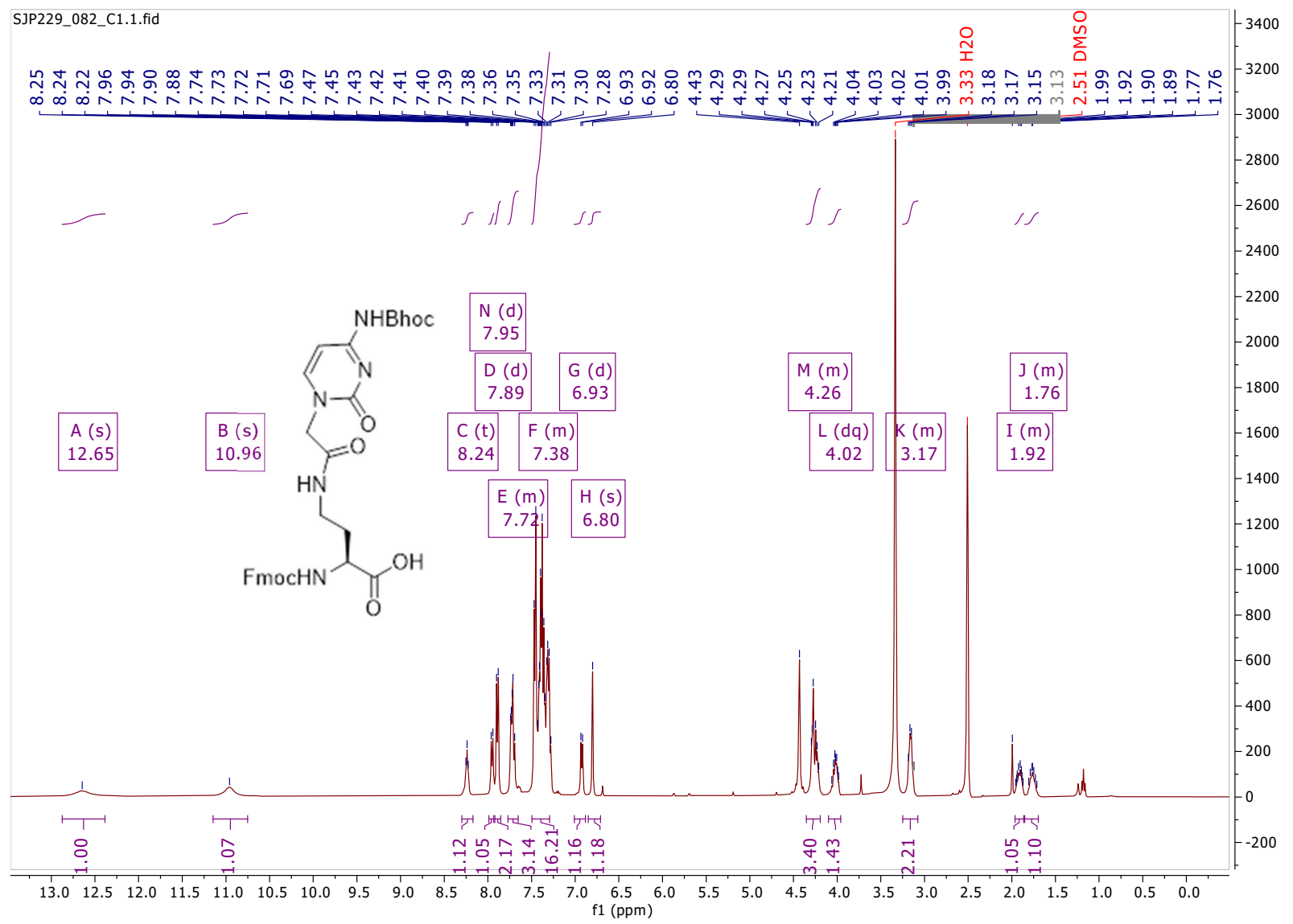




\section{${ }^{1}$ H NMR (23)}

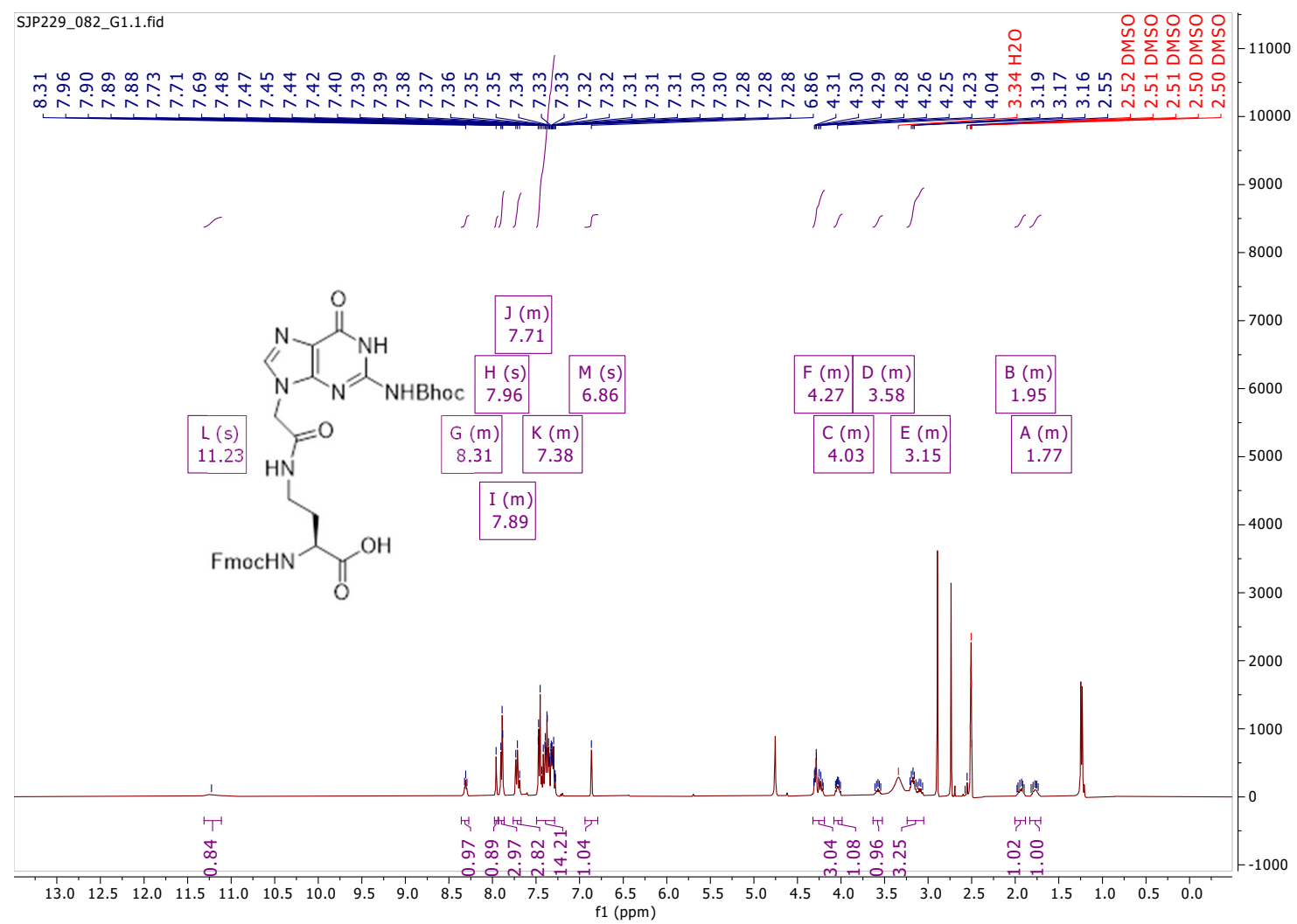

\section{${ }^{13}$ C NMR (23)}

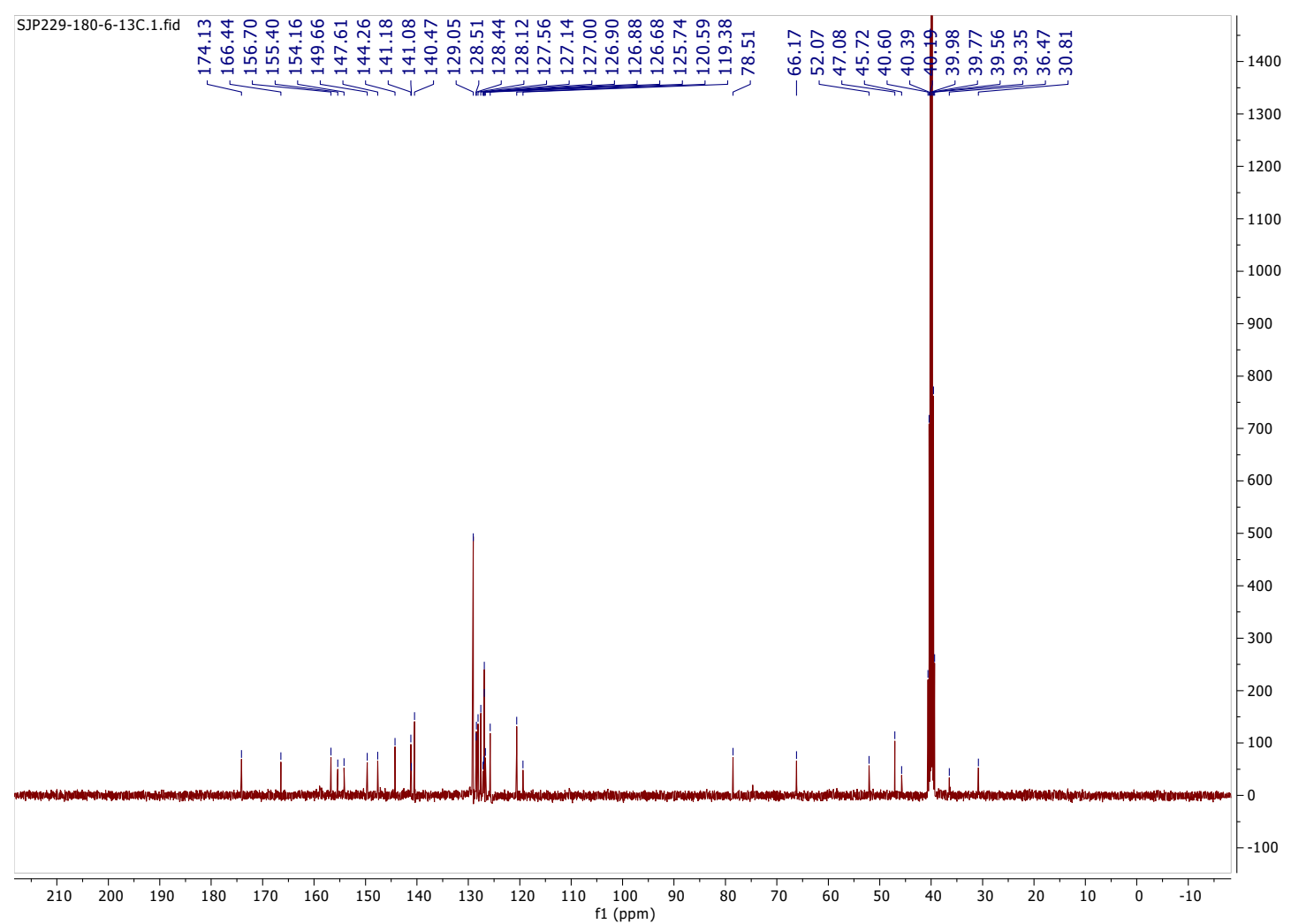




\section{${ }^{1}$ H NMR (24)}

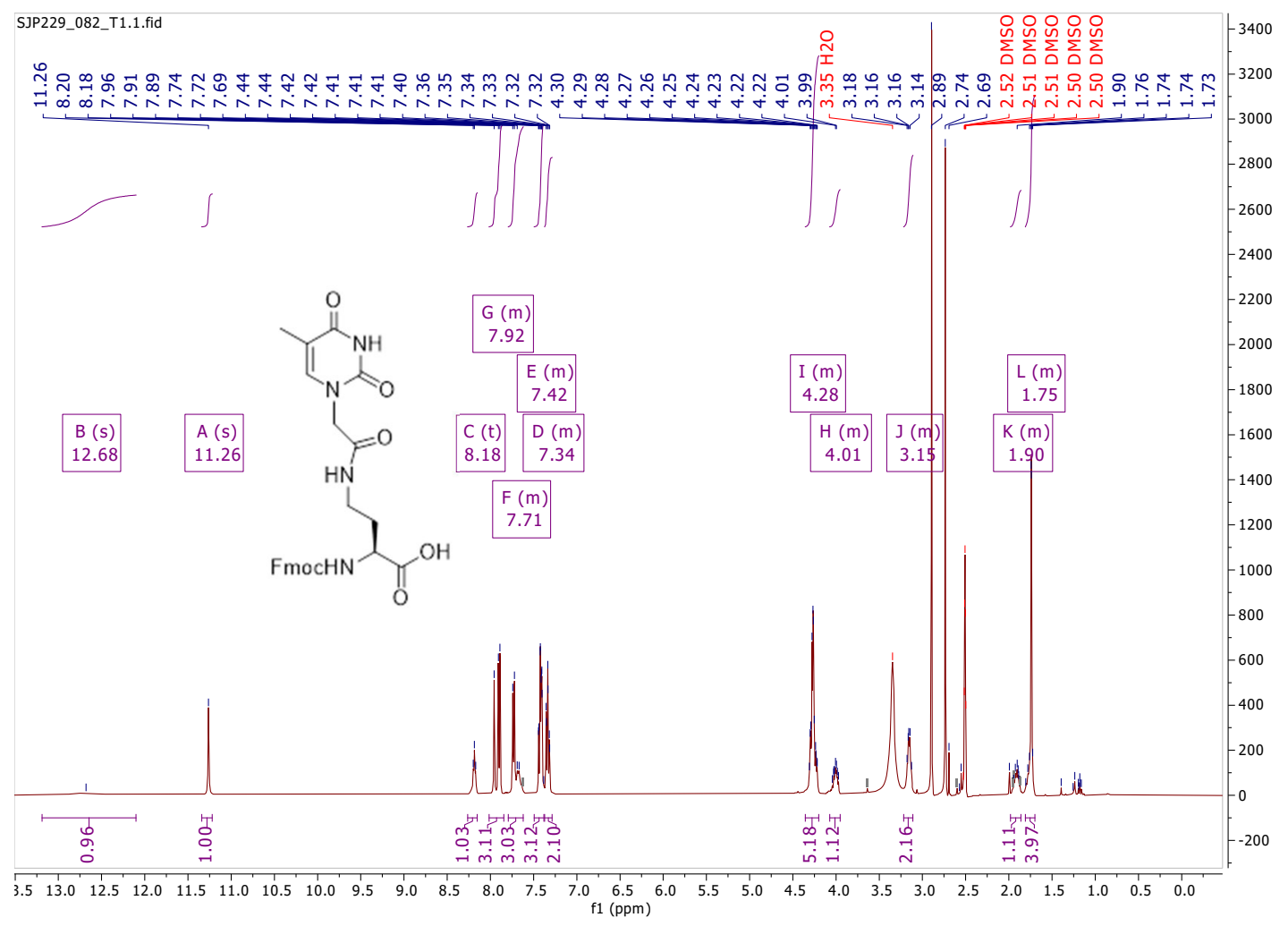


Peptide synthesis procedures

General procedure for manual solid-phase peptide synthesis (SPPS). ChemMatrix ${ }^{\circledR}$ Rink amide resin (loading $0.49 \mathrm{mmol} / \mathrm{g}$, typical scale: $200 \mathrm{mg}, 0.1 \mathrm{mmol}$ ) was loaded into a fritted syringe $(12 \mathrm{~mL})$, swollen in DMF ( $5 \mathrm{~mL}$ ) for 5 minutes and then drained. Each $\mathrm{Na}$-Fmoc protected amino acid ( $1.0 \mathrm{mmol}$, 10 equiv) was dissolved in DMF containing $0.39 \mathrm{M} \mathrm{HATU}(2.5 \mathrm{~mL}$ ). Immediately before the coupling DIEA (500 $\mu \mathrm{L}$, 30 equiv) was added to the mixture to activate the amino acid. This solution (after 15 seconds) was added to the resin and reacted for $10 \mathrm{~min}$, with occasional stirring. After completion of the coupling step, the syringe was drained, and the resin was washed with DMF $(3 \times 10 \mathrm{~mL})$. Fmoc deprotection was performed by addition of piperidine ( $20 \%$ in DMF, $5 \mathrm{~mL}$ ), to the resin $(1 \times 1 \mathrm{~min}+1 \times 5 \mathrm{~min})$, followed by draining and washing the resin with DMF $(5 \times 10 \mathrm{~mL})$. Side chain protection was as follows: Asn(Trt), Asp( $\left.\mathrm{O}^{\mathrm{t}} \mathrm{Bu}\right), \mathrm{Cys}(\mathrm{Trt})$,

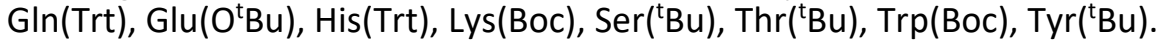

General procedure for the coupling of nucleobase monomers: for a $10 \mu \mathrm{mol}$ scale coupling: nucleobase amino acid $(50 \mu \mathrm{mol})$ and PyAOP $(50 \mu \mathrm{mol})$ were dissolved in DMF $(250 \mu \mathrm{L}, 0.1 \mathrm{M}$ reactant concentration). DIEA $(150 \mu \mathrm{mol})$ was added and the solution incubated with the peptide resin for 20 min at room temperature with occasional stirring.

Procedure for nucleobase peptide cleavage. Upon completion of the peptide synthesis, the resin was treated with a cleavage cocktail containing (v/v) 63\% TFA, 21\% TMSOTf, 4\% 1,2-ethanedithiol (EDT), $10 \%$ thioanisole and $2 \% \mathrm{cresol}(\mathrm{v} / \mathrm{v})$ at room temperature for $1 \mathrm{~h}$. The TFA volume was then reduced under $\mathrm{N}_{2}$ stream and cold diethyl ether $\left(-78^{\circ} \mathrm{C}\right)$ was added to precipitate the peptide. The resulting suspension was centrifugated at $4000 \mathrm{rpm}$ for $3 \mathrm{~min}$ and the liquid was discarded. After repeating this step twice more, the pellet was dissolved in Milli-Q ultrapure water $+0.1 \%$ TFA and lyophilized.

Procedure for peptide cleavage. Upon completion of the peptide synthesis, the resin was treated with a cleavage cocktail containing 94\% TFA, 2.5\% water, 2.5\% 1,2-ethanedithiol (EDT) and 1\% triisopropylsilane (TIPS) (v/v) at room temperature for $2 \mathrm{~h}$. The TFA volume was then reduced under $\mathrm{N}_{2}$ stream and cold diethyl ether $\left(-80^{\circ} \mathrm{C}\right)$ was added to precipitate the peptide. The resulting suspension was centrifugated at $4000 \mathrm{rpm}$ for $3 \mathrm{~min}$ and the liquid was discarded. After repeating this step twice more, the pellet was dissolved in Milli-Q ultrapure water + 0.1\% TFA and lyophilized.

General procedure for solid-phase extraction (SPE) purification. Peptides were desalted using C18 SPE cartridges according to general procedures from Agilent Sample Prep Solutions Bond Elut.

Preparation of peptide solutions. Weight of lyophilized peptide was determined with analytical scale. The peptide's molecular weight plus $n \times 114$ ( $n=$ number of $K, R, H$ and $N$-term, $114=m w$ TFA) was used to calculated number of moles. In case of the libraries the average mw of all used monomers was multiplied by the number of variable positions. The peptide was than dissolved in a volume according to the desired final concentration.

Determination of oligonucleotide concentration. The concentration of oligonulceotides was calculated using the Beer-Lambert law by measuring the absorbance of the oligo sample at $260 \mathrm{~nm}$. The absorbance was measured by averaging at least two independent readings of the same sample on a BioTek Synergy HT plate reader outfitted with a BioTek Take 3 micro-volume plate. 
Nucleobase peptide and peptide characterization

Test of nucleobase monomers incorporation into short peptide by SPPS

Individual purities were calculated by integration of relevant peaks in the LCMS TIC chromatograms

Nucleobase peptide 26

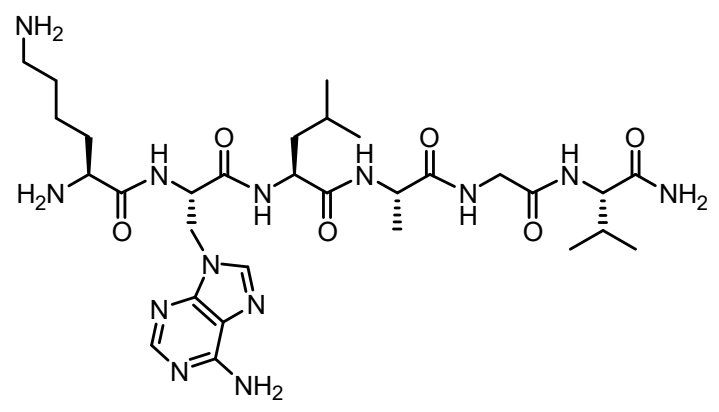

HRMS (SI-QToF) $m / z$ : [M+H] ${ }^{+}$calcd. 690.416 found 690.419
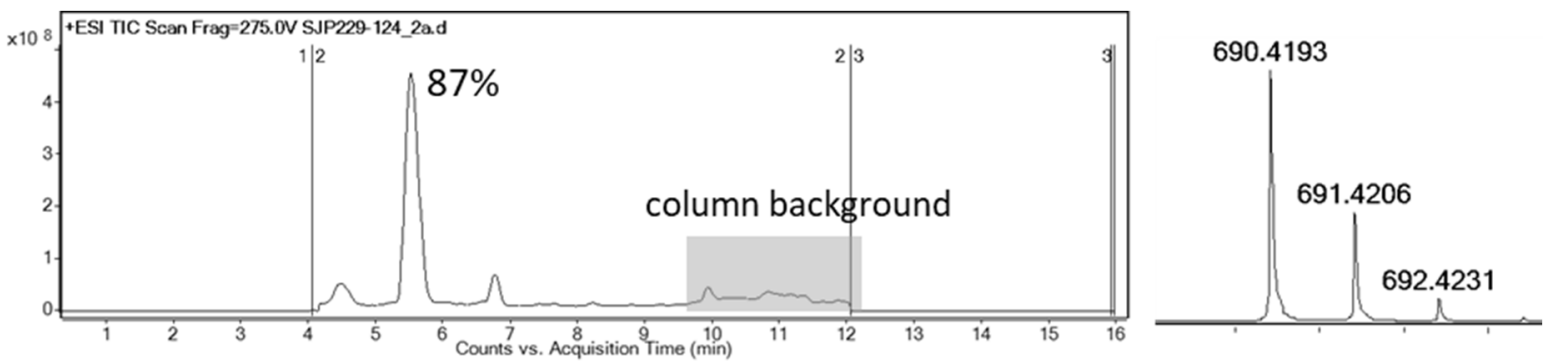

Nucleobase peptide 27

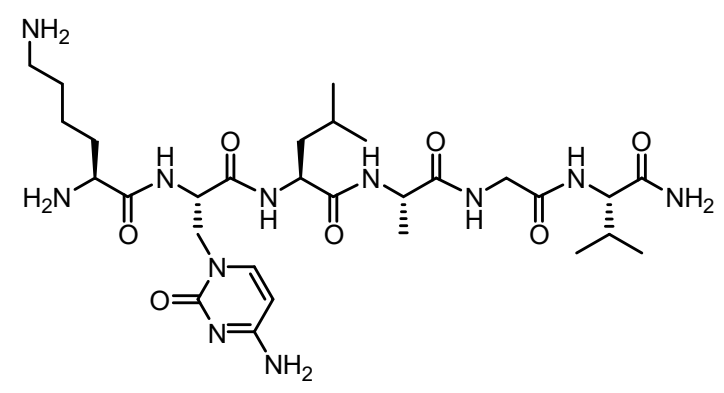

HRMS (SI-QToF) $m / z$ : [M+H] $]^{+}$calcd. 666.405 found 666.408 


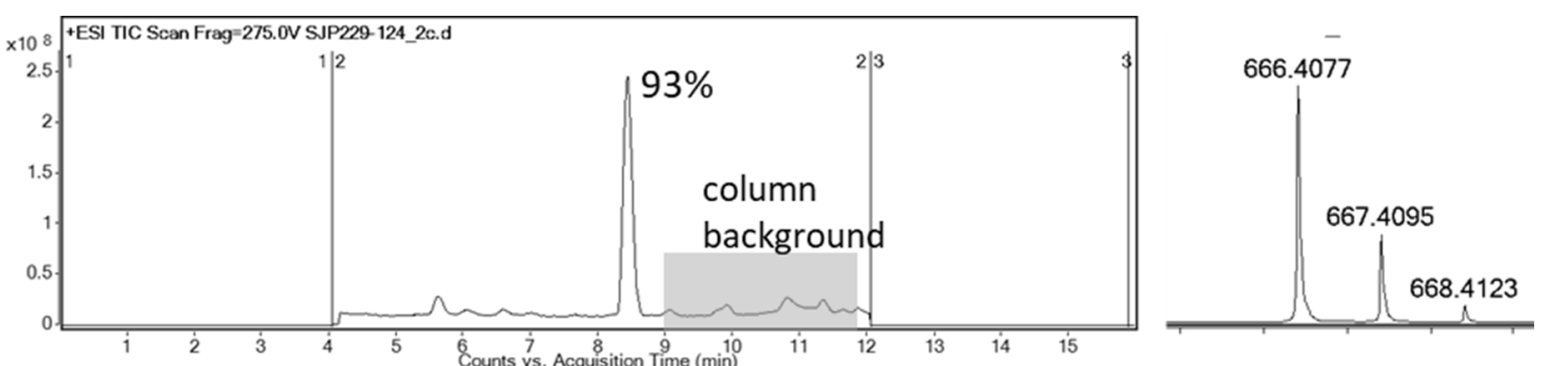




\section{Nucleobase peptide 28}<smiles>CC(C)CC(NC(=O)[C@H](Cn1cnc2c(=O)[nH]c(N)nc21)NC(=O)C(N)CCCCN)C(=O)N[C@@H](C)C(=O)NCC(=O)N[C@H](C(N)=O)C(C)C</smiles>

HRMS (SI-QToF) $m / z$ : $[\mathrm{M}+\mathrm{H}]^{+}$calcd. 706.411 found 706.415

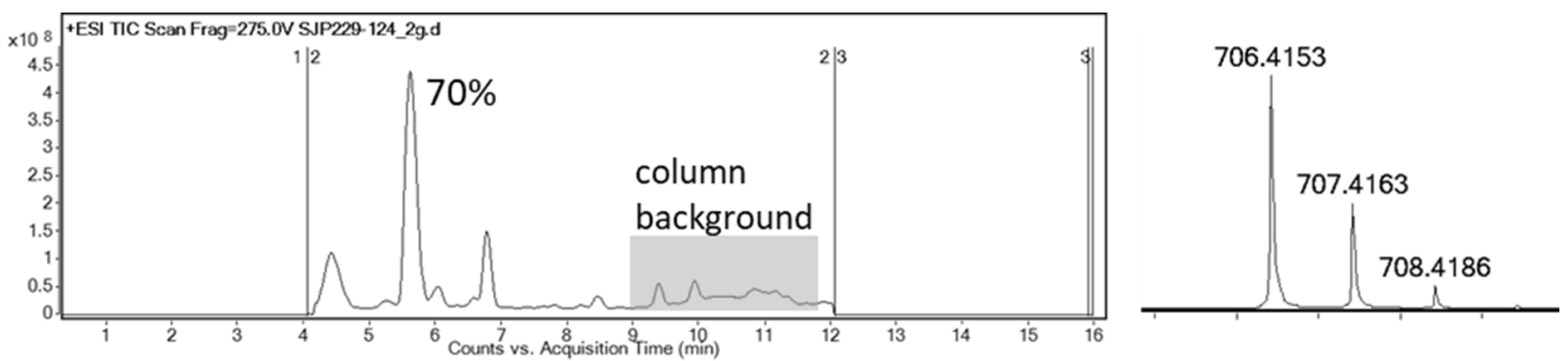

Nucleobase peptide 29<smiles>Cc1cn(C[C@H](NC(=O)C(N)CCCCN)C(=O)NC(CC(C)C)C(=O)N[C@@H](C)C(=O)NCC(=O)N[C@@H](C(N)=O)C(C)C)c(=O)[nH]c1=O</smiles>

HRMS (SI-QToF) $m / z$ : [M+H] ${ }^{+}$calcd. 681.402 found 681.407

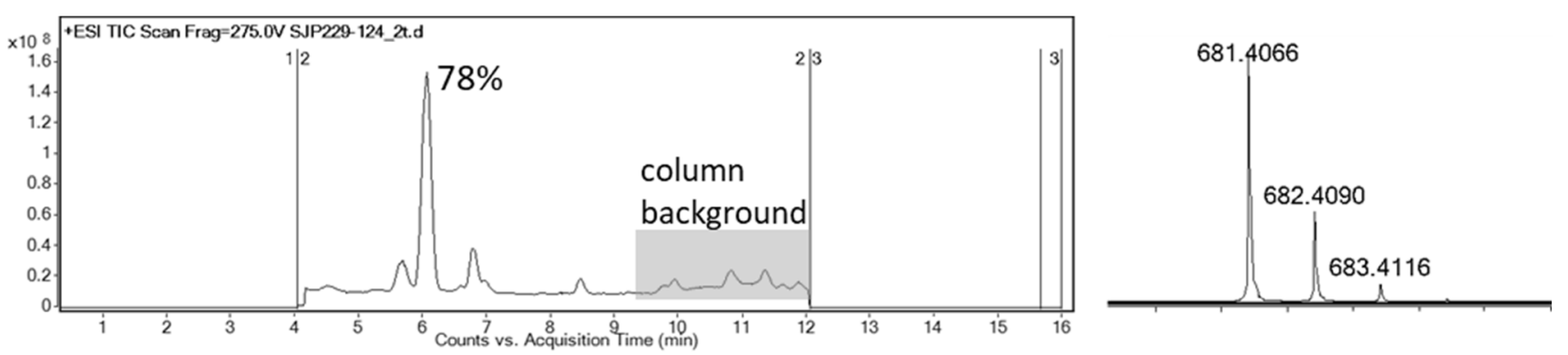




\section{Nucleobase peptide 30}<smiles>CC(C)CC(NC(=O)[C@H](CCn1cnc2c(N)ncnc21)NC(=O)C(N)CCCCN)C(=O)N[C@@H](C)C(=O)NCC(=O)N[C@H](C(N)=O)C(C)C</smiles>

HRMS (SI-QToF) $m / z$ : $[\mathrm{M}+\mathrm{H}]^{+}$calcd. 704.432 found 704.433
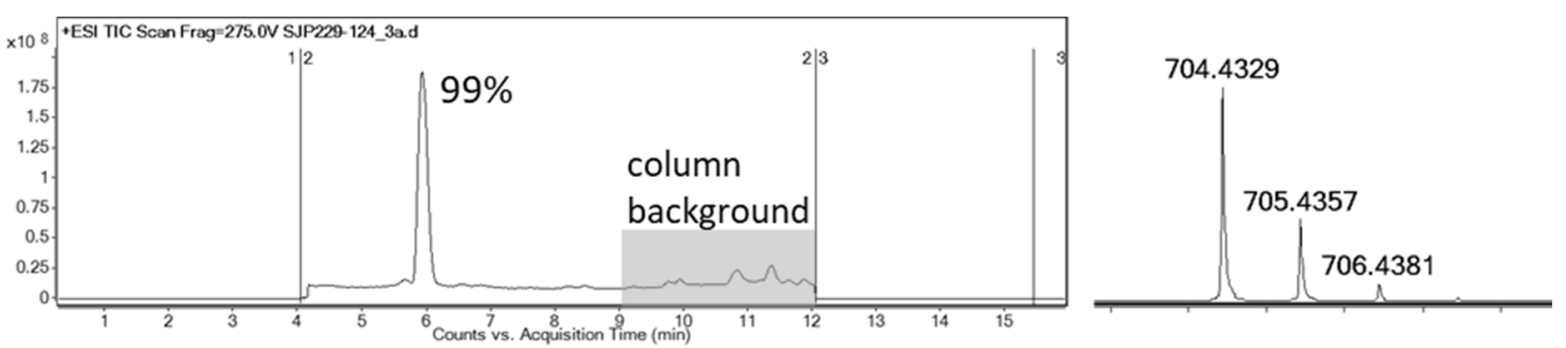

Nucleobase peptide 31<smiles>CC(C)CC(NC(=O)[C@H](CCn1ccc(N)nc1=O)NC(=O)C(N)CCCCN)C(=O)N[C@@H](C)C(=O)NCC(=O)N[C@H](C(N)=O)C(C)C</smiles>

HRMS (SI-QToF) $m / z$ : $[\mathrm{M}+\mathrm{H}]^{+}$calcd. 680.421 found 680.428
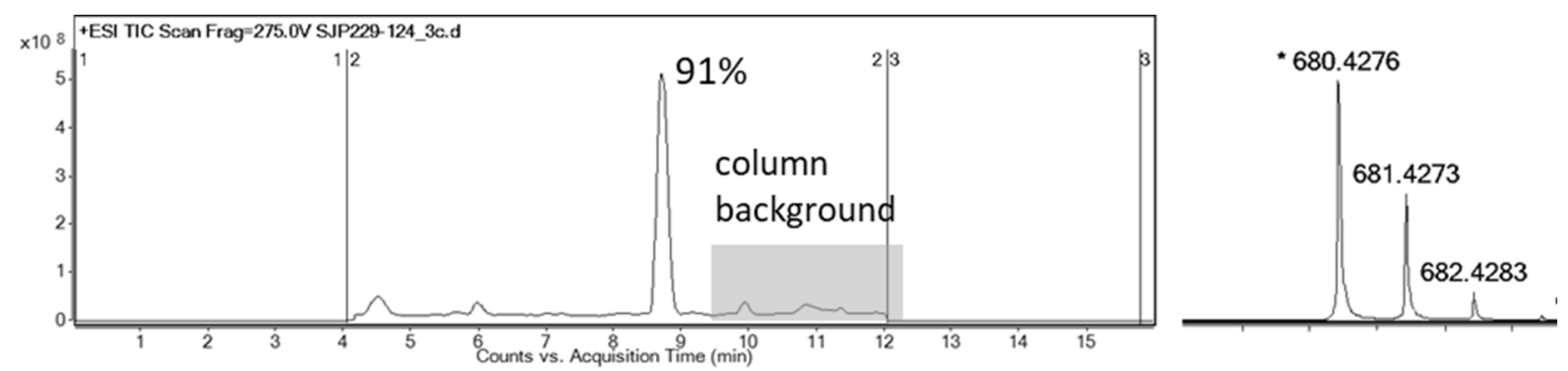


\section{Nucleobase peptide 32}<smiles>CC(C)CC(NC(=O)[C@H](CCn1cnc2c(=O)[nH]c(N)nc21)NC(=O)C(N)CCCCN)C(=O)N[C@@H](C)C(=O)NCC(=O)N[C@H](C(N)=O)C(C)C</smiles>

HRMS (SI-QToF) $m / z$ : $[\mathrm{M}+\mathrm{H}]^{+}$calcd. 720.427 found 720.430
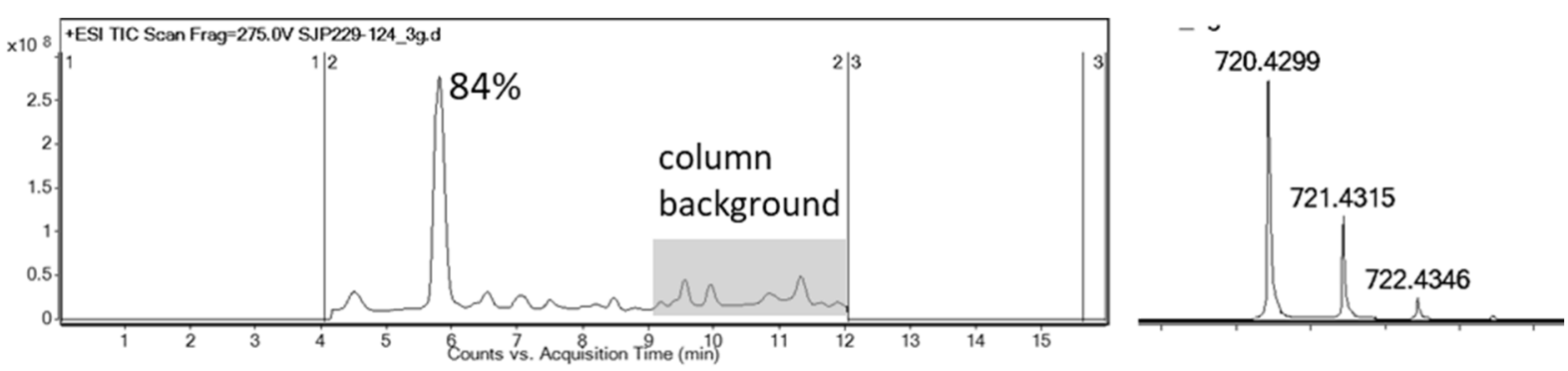

\section{Nucleobase peptide 33}

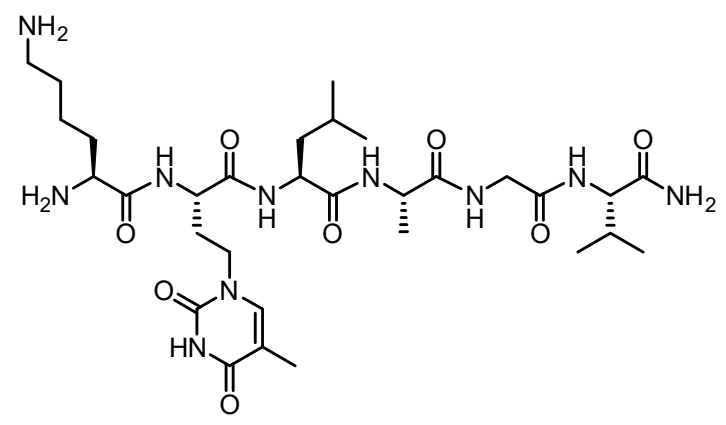

HRMS (SI-QToF) m/z: [M+H] $]^{+}$calcd. 695.420 found 695.424

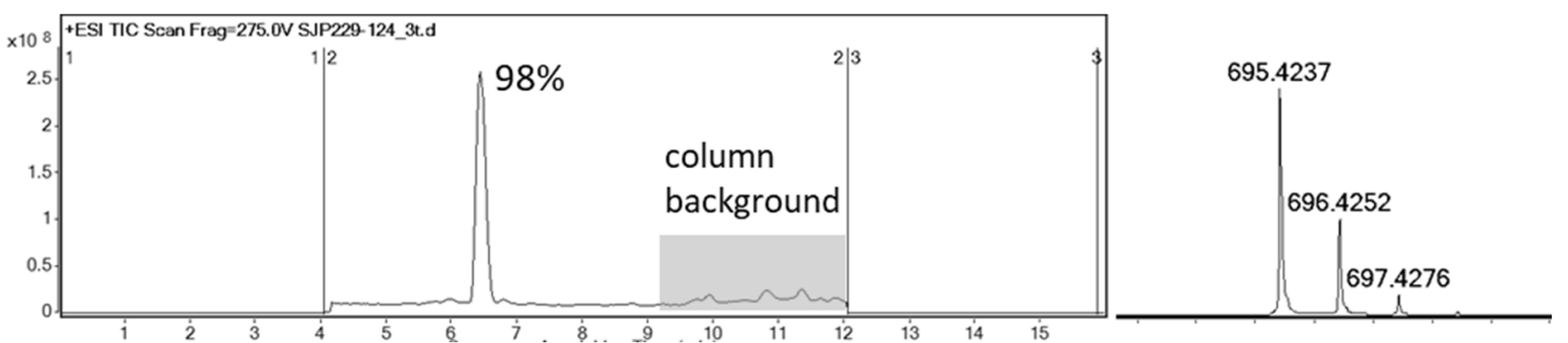




\section{Nucleobase peptide 34}<smiles>CC(C)CC(NC(=O)[C@H](CCNC(=O)Cn1cnc2c(N)ncnc21)NC(=O)C(N)CCCCN)C(=O)N[C@@H](C)C(=O)NCC(=O)NC(C(N)=O)C(C)C</smiles>

HRMS (SI-QToF) $m / z$ : $[\mathrm{M}+\mathrm{H}]^{+}$calcd. 761.454 found 761.454
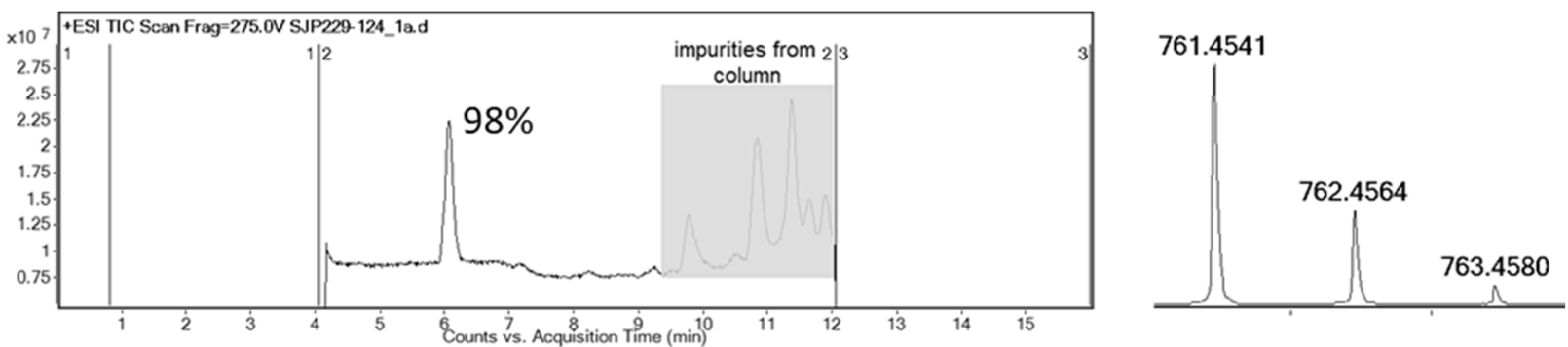

Nucleobase peptide 35<smiles>CC(C)CC(NC(=O)[C@H](CCNC(=O)Cn1ccc(N)nc1=O)NC(=O)C(N)CCCCN)C(=O)N[C@@H](C)C(=O)NCC(=O)NC(C(N)=O)[C@@H](C)C(C)C</smiles>

HRMS (SI-QToF) $m / z:[M+H]^{+}$calcd. 737.442 found 737.444
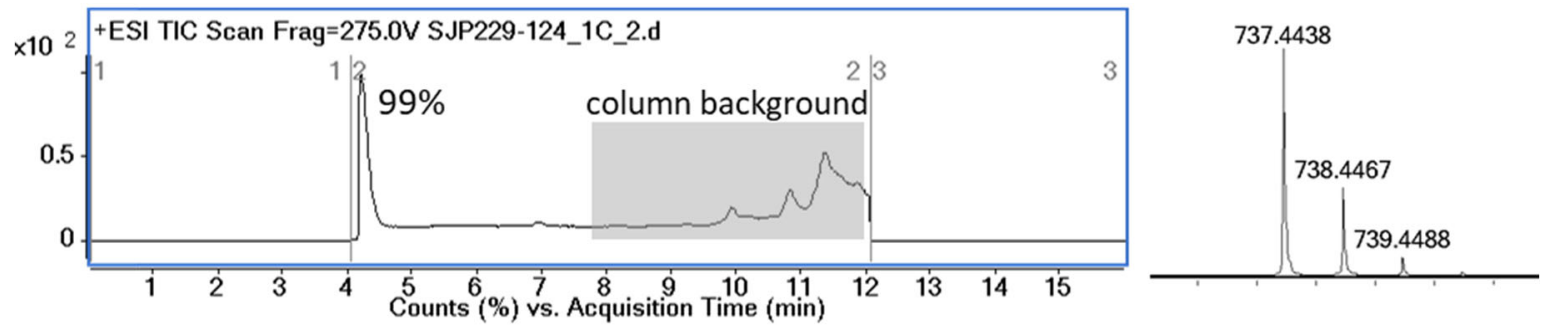
Nucleobase peptide 36<smiles>CC(C)CC(NC(=O)[C@H](CCNC(=O)Cn1cnc2c(=O)[nH]c(N)nc21)NC(=O)C(N)CCCCN)C(=O)N[C@@H](C)C(=O)NCC(=O)NC(C(N)=O)C(C)C</smiles>

HRMS (SI-QToF) m/z: [M+H] ${ }^{+}$calcd. 777.448 found 777.449
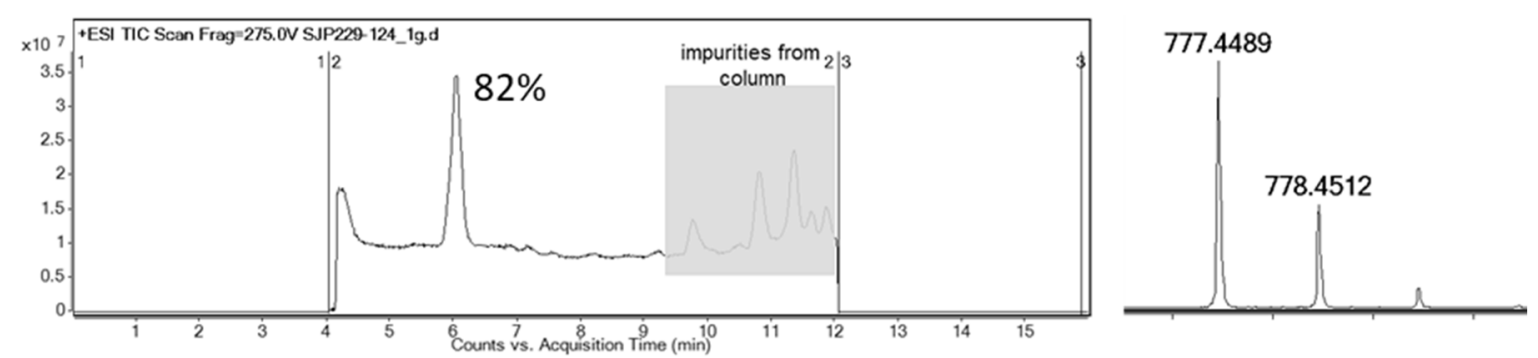

\section{Nucleobase peptide 37}<smiles>Cc1cn(CC(=O)NCC[C@H](NC(=O)C(N)CCCCN)C(=O)NC(CC(C)C)C(=O)N[C@@H](C)C(=O)NCC(=O)NC(C(N)=O)C(C)C)c(=O)[nH]c1=O</smiles>

HRMS (SI-QToF) $m / z$ : [M+H] $]^{+}$calcd. 752.442 found 752.447
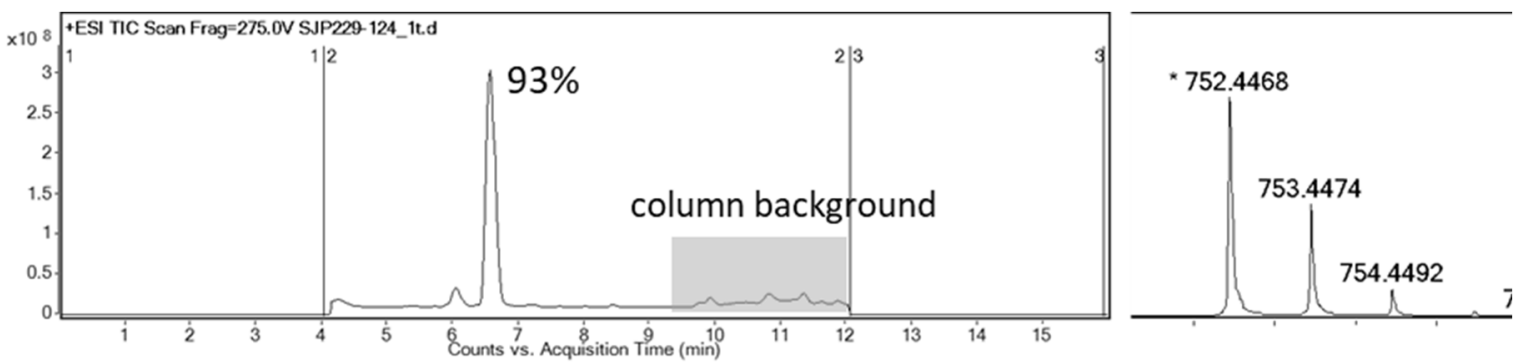


\section{Peptide 38}

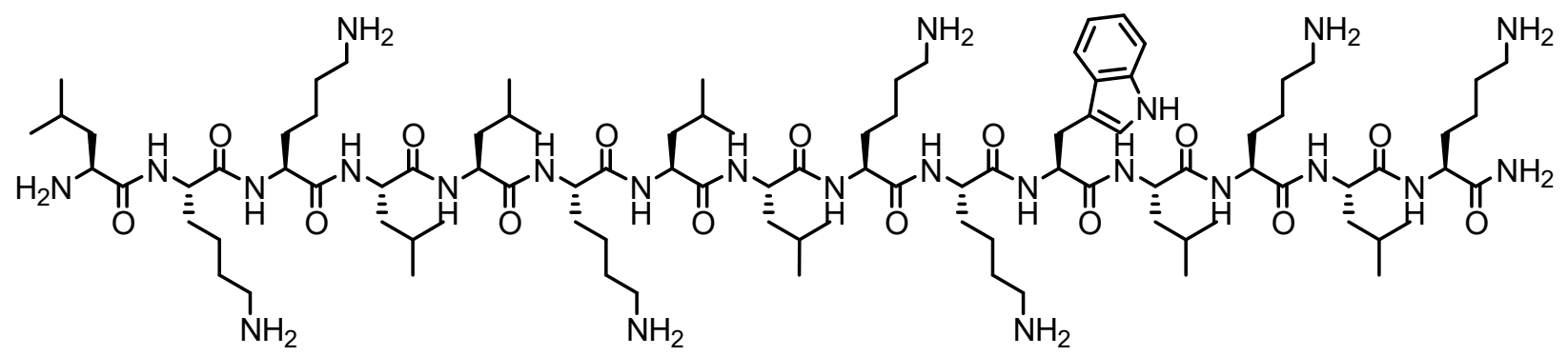

HRMS (SI-QToF) m/z: [M+H] ${ }^{+}$calcd. 1892.37 found 1892.44
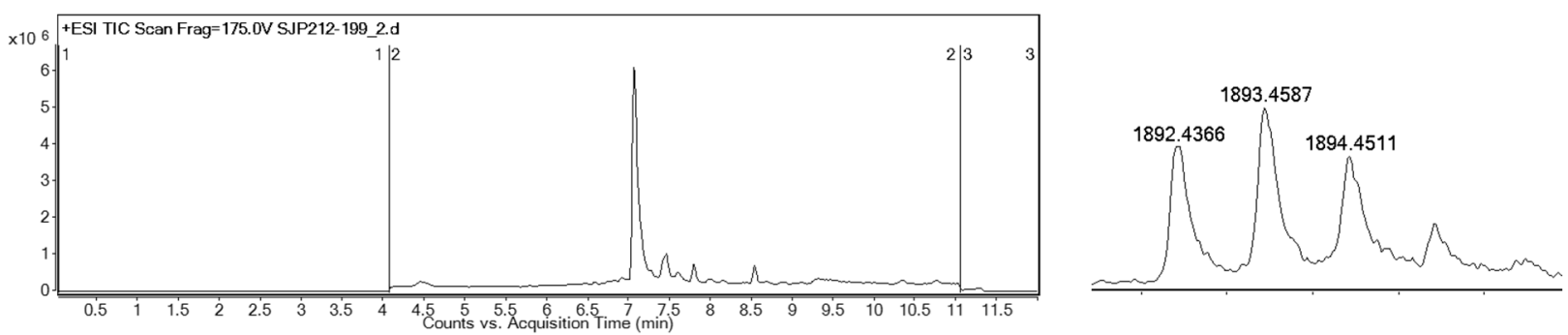

\section{Biotin}

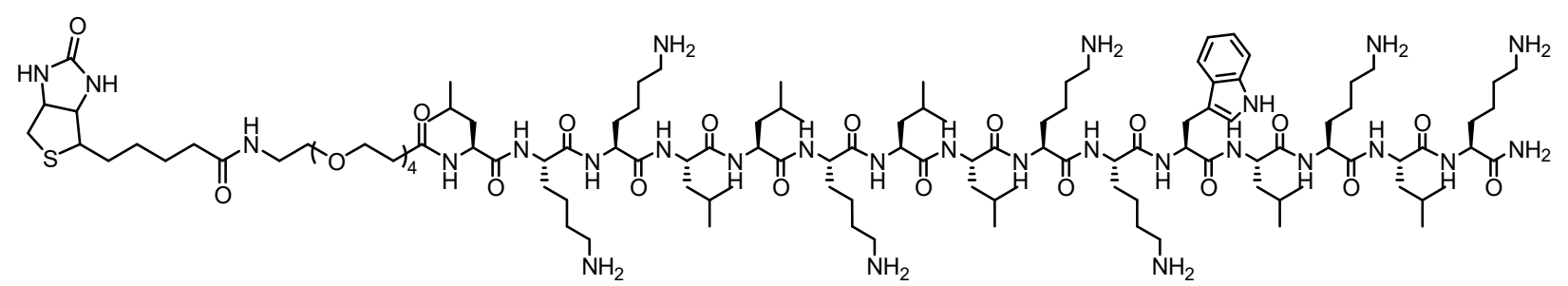

HRMS (SI-QToF) $m / z$ : $[\mathrm{M}+5 \mathrm{H}]^{5+}$ calcd. 473.924 found 473.926

$[\mathrm{M}+4 \mathrm{H}]^{4+}$ calcd. 592.153 found 591.154

$[\mathrm{M}+3 \mathrm{H}]^{3+}$ calcd. 789.201 found 780.201
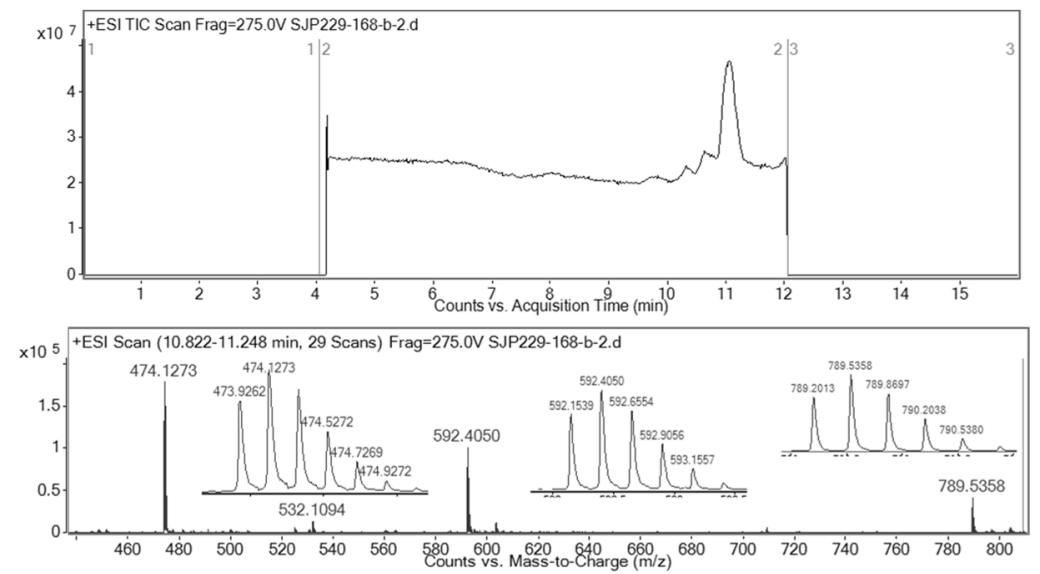


\section{Nucleobase peptide 39}<smiles>CC(C)C[C@H](NC(=O)[C@H](CCNC(=O)Cn1cnc2c(N)ncnc21)NC(=O)[C@H](CC(=O)O)NC(=O)[C@H](CCCNC(=N)N)NC(=O)[C@H](CCn1cnc2c(N)ncnc21)NC(=O)[C@H](CCNC(=O)Cn1cnc2c(=O)[nH]c(N)nc21)NC(=O)[C@H](N)CCCCN)C(=O)N[C@@H](CCn1cnc2c(=O)[nH]c(N)nc21)C(=O)N[C@@H](C)C(=O)N[C@@H](CCCCN)C(N)=O</smiles>

HRMS (SI-QToF) $m / z$ : $[\mathrm{M}+5 \mathrm{H}]^{4+}$ calcd. 350.381 found 350.382

$[\mathrm{M}+4 \mathrm{H}]^{4+}$ calcd. 437.724 found 437.726

$[\mathrm{M}+3 \mathrm{H}]^{3+}$ calcd. 583.296 found 583.297
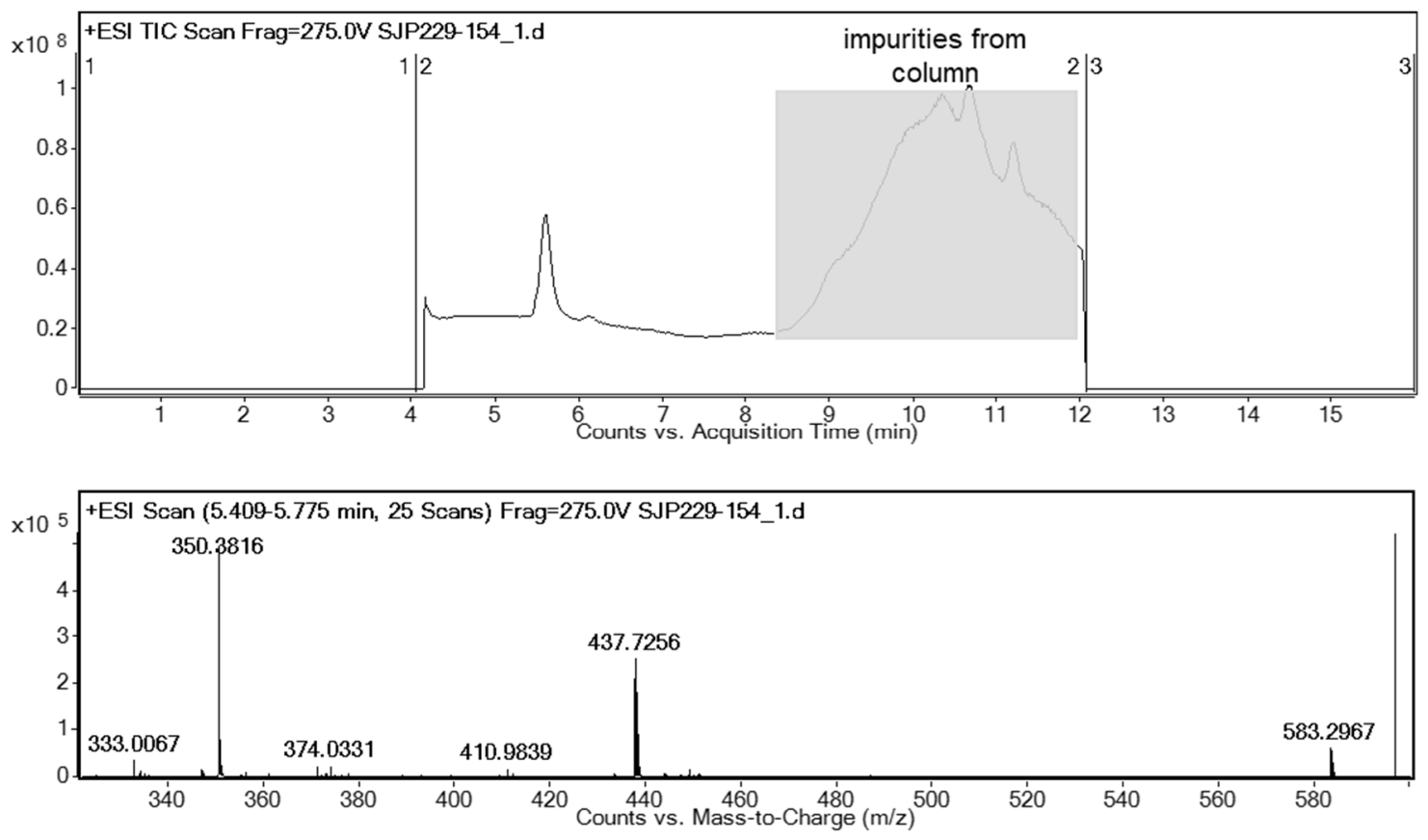


\section{Nucleobase peptide 40}<smiles>CC(C)CC(NC(=O)[C@H](CCNC(=O)Cn1cnc2c(N)ncnc21)NC(=O)[C@H](CC(=O)O)NC(=O)[C@H](CCCNC(=N)N)NC(=O)[C@H](CCn1cnc2c(N)ncnc21)NC(=O)[C@H](CCNC(=O)Cn1cnc2c(=O)[nH]c(N)nc21)NC(=O)[C@H](N)CCCCN)C(=O)N[C@@H](CCn1cnc2c(=O)[nH]c(N)nc21)C(=O)N[C@@H](C)C(=O)N[C@@H](CCCCN)C(=O)NCCC(=O)N[C@@H](CCCCNC(=O)CCCC[C@@H]1SC[C@@H]2NC(=O)N[C@@H]12)C(N)=O</smiles>

HRMS (SI-QToF) $m / z$ : $[\mathrm{M}+5 \mathrm{H}]^{5+}$ calcd. 435.423 found 435.421

$[\mathrm{M}+4 \mathrm{H}]^{4+}$ calcd. 544.027 found 544.024

$[\mathrm{M}+3 \mathrm{H}]^{3+}$ calcd. 725.0326 found 725.030

$[\mathrm{M}+2 \mathrm{H}]^{2+}$ calcd. 1087.04 found 1087.04
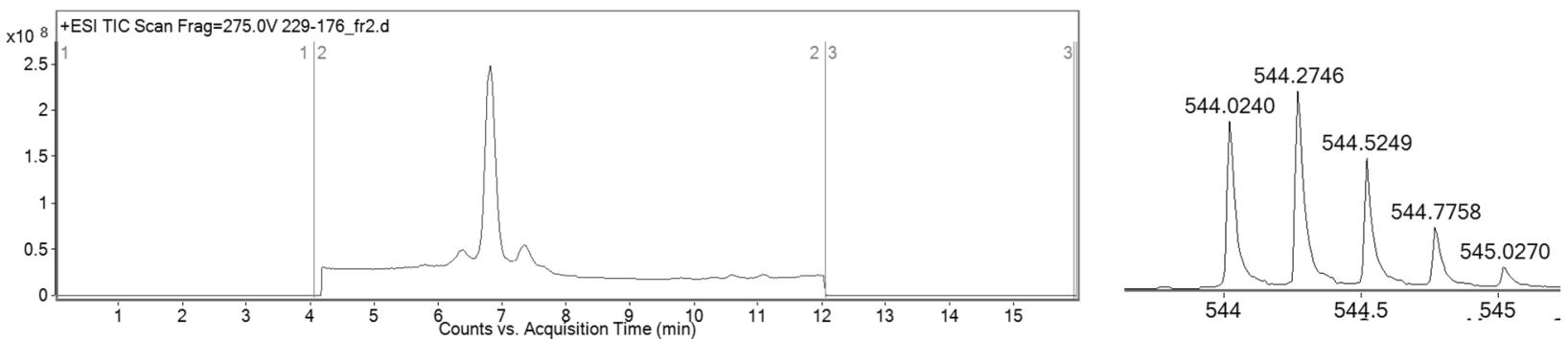


\section{Alanine scan}

Original sequence: 39Biotin

Lys - DabG - hAlaA - Arg - Asp -DabA - Leu -DabG - Ala - Lys - bAla - Lys(Biotin)-CONH 2

Nucleobase peptides were synthesized according to the general SPPS procedure, cleaved and used without further purification. Main product in each chromatogram is indicated with *.

AlaScan-1:

Ala - DabG - hAlaA - Arg - Asp -DabA - Leu -hAlaG - Ala - Lys - $\beta$ Ala - Lys(Biotin)-CONH 2

HRMS (SI-QToF) $m / z:[\mathrm{M}+4 \mathrm{H}]^{4+}$ calcd. 529.762 found 529.762
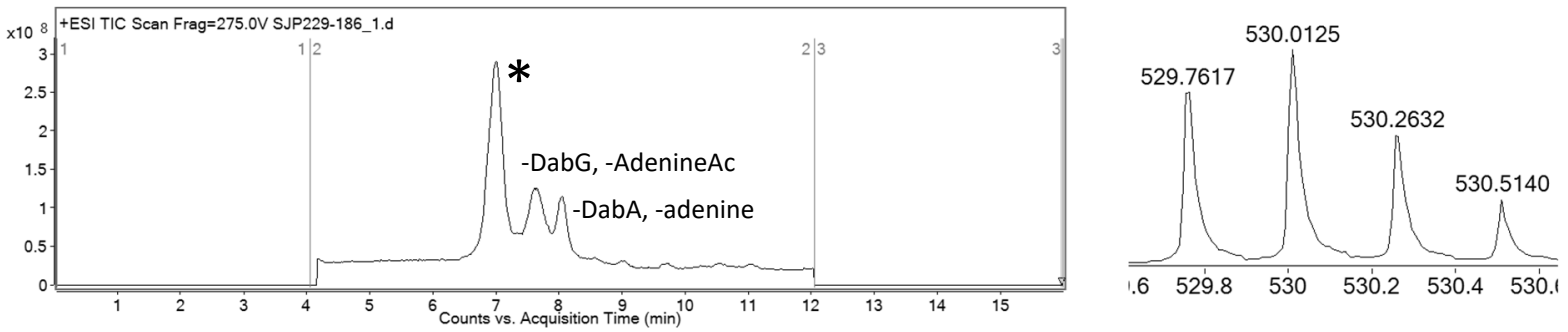

\section{AlaScan-2:}

Lys - Ala - hAlaA - Arg - Asp -DabA - Leu - hAlaG - Ala - Lys - BAla - Lys(Biotin)-CONH 2

HRMS (SI-QToF) $m / z$ : $[\mathrm{M}+4 \mathrm{H}]^{4+}$ calcd. 489.009 found 489.010
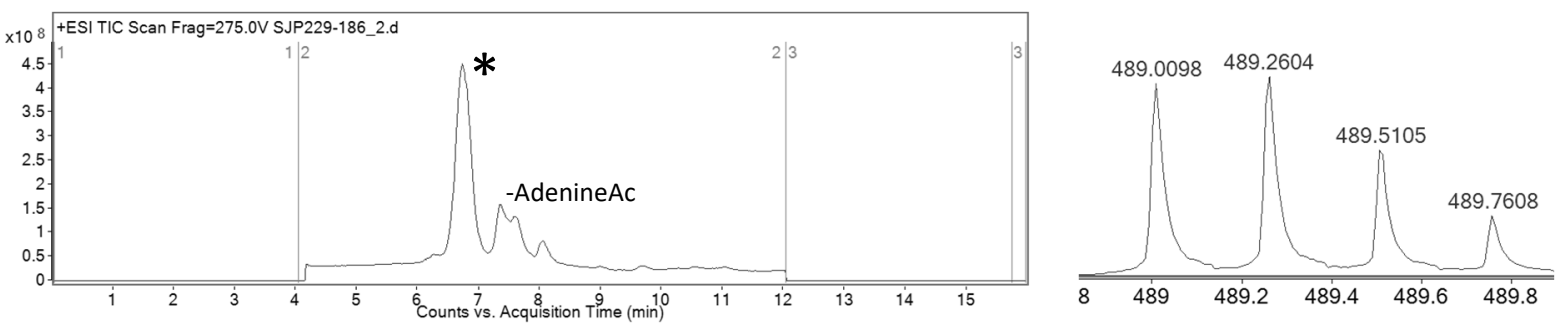

AlaScan-3:

Lys - DabG - Ala - Arg - Asp -DabA - Leu - hAlaG - Ala - Lys - BAla - Lys(Biotin)-CONH HRMS (SI-QToF) m/z: [M+4H] ${ }^{4+}$ calcd. 507.263 found 507.264
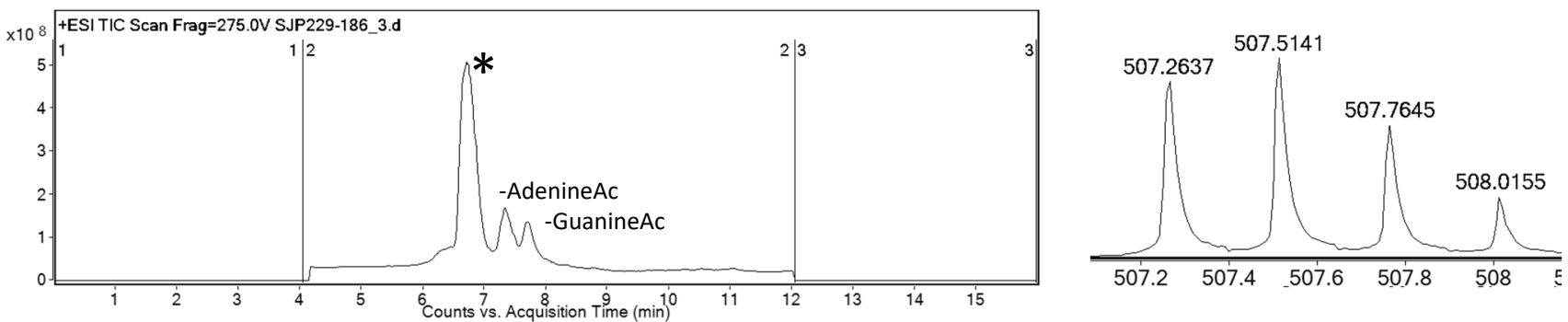


\section{AlaScan-4:}

Lys - DabG - hAlaA - Ala- Asp -DabA - Leu - hAlaG - Ala - Lys - BAla - Lys(Biotin)-CONH 2 HRMS (SI-QToF) $m / z:[\mathrm{M}+4 \mathrm{H}]^{4+}$ calcd. 522.761 found 522.762
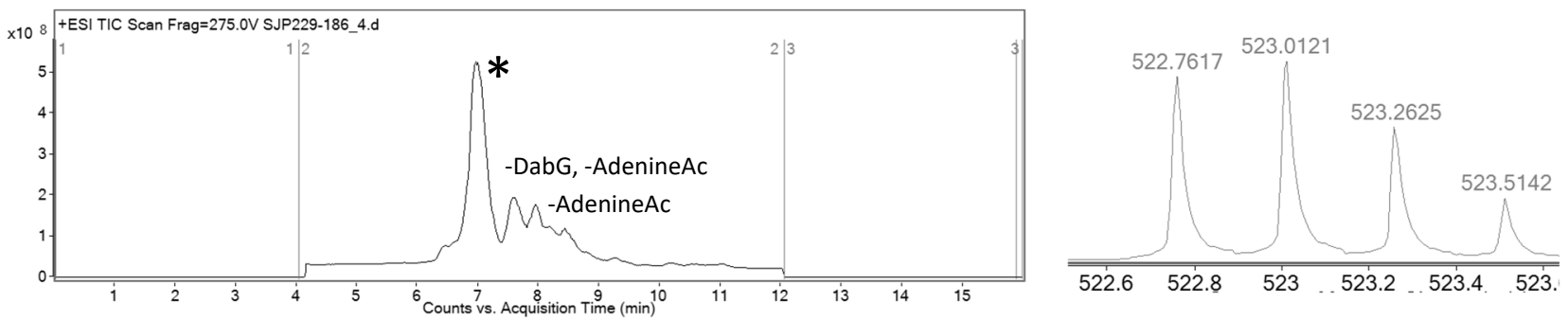

\section{AlaScan-5:}

Lys - DabG - hAlaA - Arg - Ala -DabA - Leu - hAlaG - Ala - Lys - BAla - Lys(Biotin)-CONH 2

HRMS (SI-QToF) $m / z$ : $[\mathrm{M}+5 \mathrm{H}]^{5+}$ calcd. 426.625 found 426.626
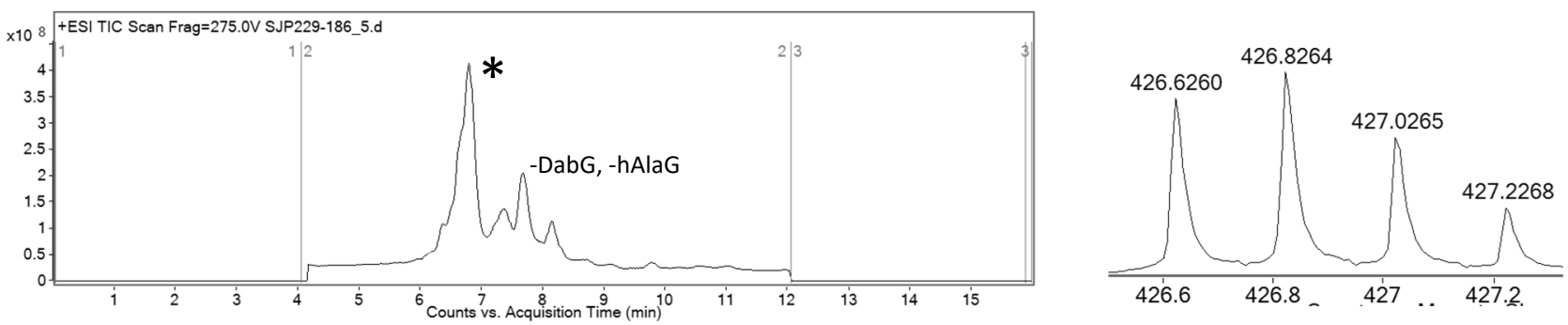

\section{AlaScan-6:}

Lys - DabG - hAlaA - Arg - Asp -Ala - Leu - hAlaG - Ala - Lys - BAla - Lys(Biotin)-CONH 2

HRMS (SI-QToF) $m / z$ : $[\mathrm{M}+4 \mathrm{H}]^{4+}$ calcd. 493.008 found 493.009
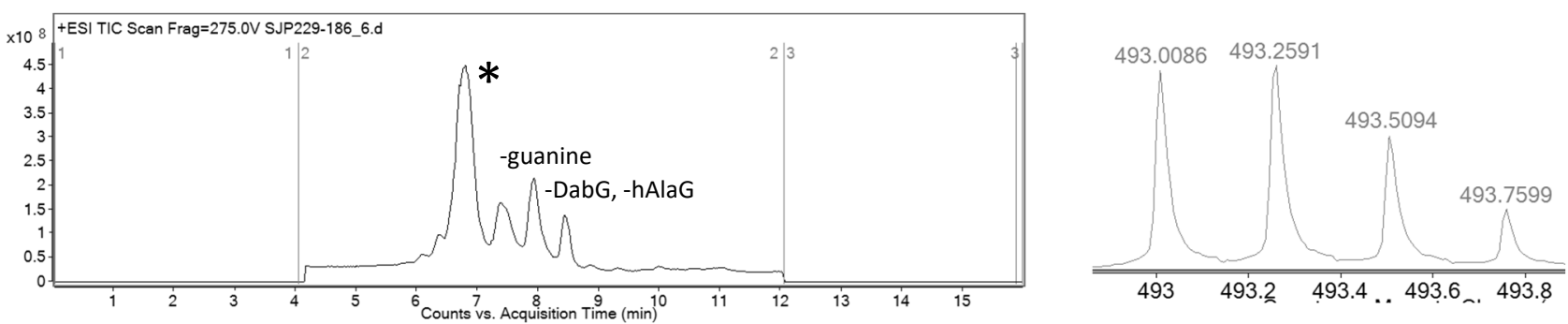


\section{AlaScan-7:}

Lys - DabG - hAlaA - Arg - Asp -DabA - Ala - hAlaG - Ala - Lys - BAla - Lys(Biotin)-CONH 2 HRMS (SI-QToF) $m / z:[\mathrm{M}+4 \mathrm{H}]^{4+}$ found 533.766
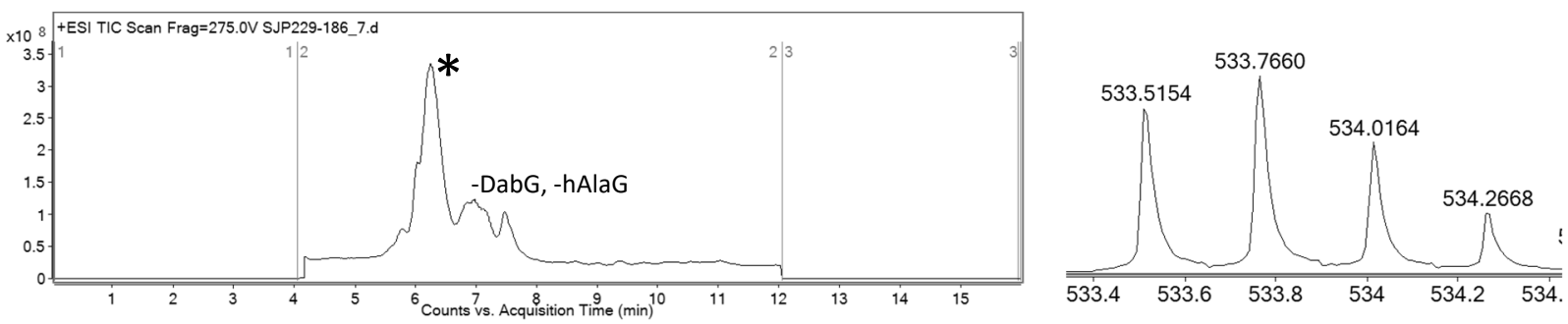

\section{AlaScan-8:}

Lys - DabG - hAlaA - Arg - Asp -DabA - Leu - Ala - Ala - Lys - BAla - Lys(Biotin)-CONH 2

HRMS (SI-QToF) $m / z:[\mathrm{M}+4 \mathrm{H}]^{4+}$ calcd. 503.264 found 503.265
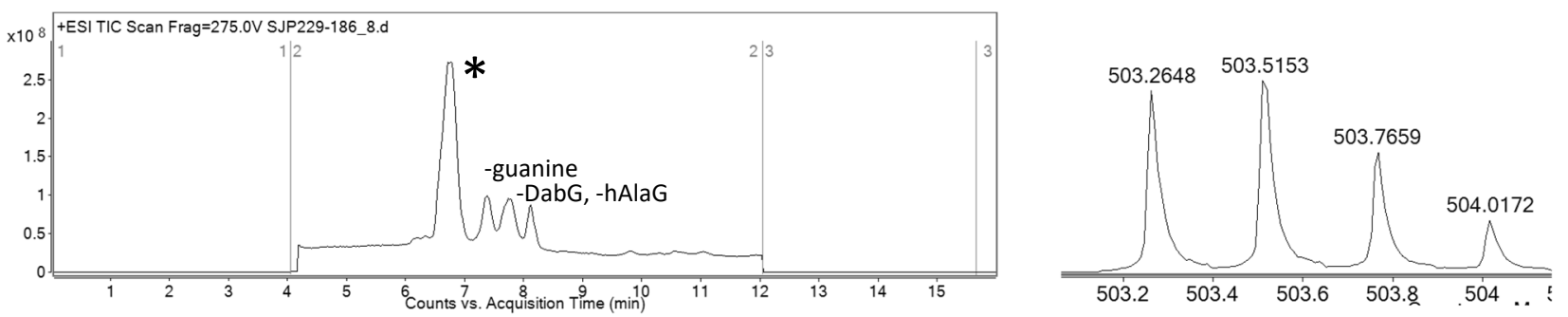

\section{AlaScan-9:}

Lys - DabG - hAlaA - Arg - Asp -DabA - Leu - hAlaG - Ala - Ala - BAla - Lys(Biotin)-CONH 2 HRMS (SI-QToF) $m / z:[\mathrm{M}+4 \mathrm{H}]^{4+}$ calcd. 526.762 found 526.763
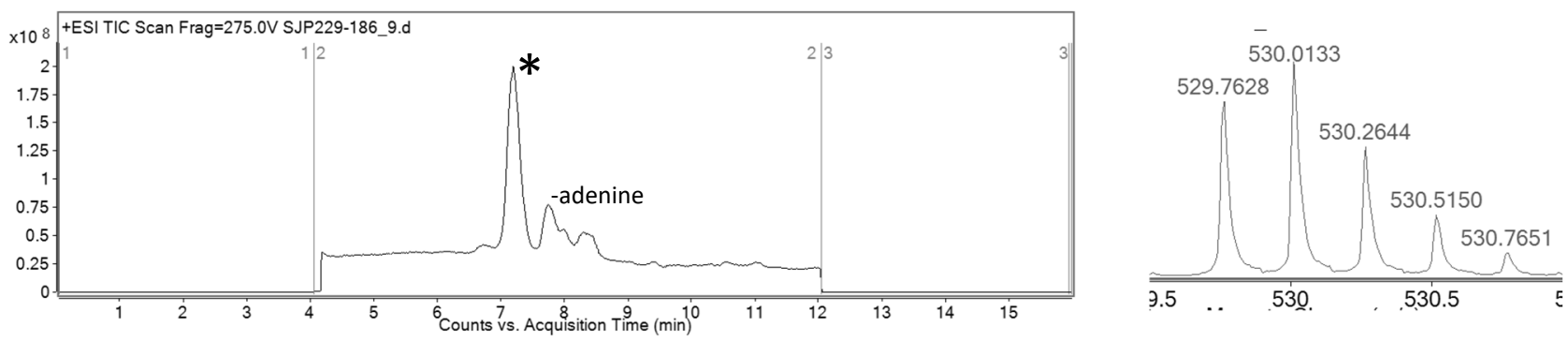


\section{Mutation of all 4 nucleobases}

\section{Original sequence: 39}

Lys - DabG - hAlaA - Arg - Asp -DabA - Leu -DabG - Ala - Lys - bAla - Lys(Biotin)-CONH 2

Peptides were synthesized according to the general SPPS procedure, cleaved and used without further purification

Tetra-Ala-mutant

Lys - Ala - Ala - Arg - Asp - Ala - Leu - Ala - Ala - Lys - bAla - Lys(Biotin)-CONH 2

HRMS (SI-QToF) $m / z:[M+4 H]^{4+}$ calcd. 360.464 found 360.265
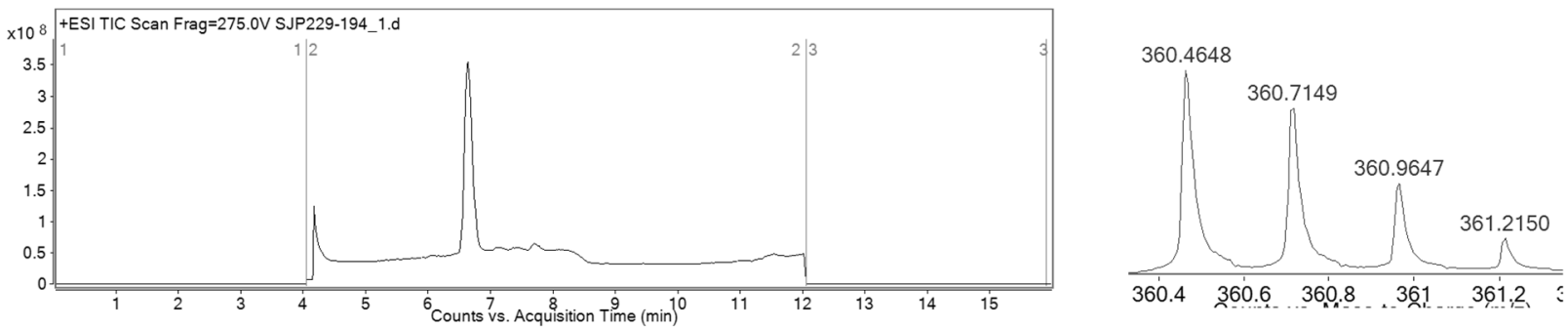

Tetra-Trp-mutant

Lys - Trp - Trp - Arg - Asp - Trp - Leu - Trp - Ala - Lys - bAla - Lys(Biotin)-CONH 2

HRMS (SI-QToF) $m / z$ : $[\mathrm{M}+4 \mathrm{H}]^{4+}$ calcd. 475.509 found 475.506
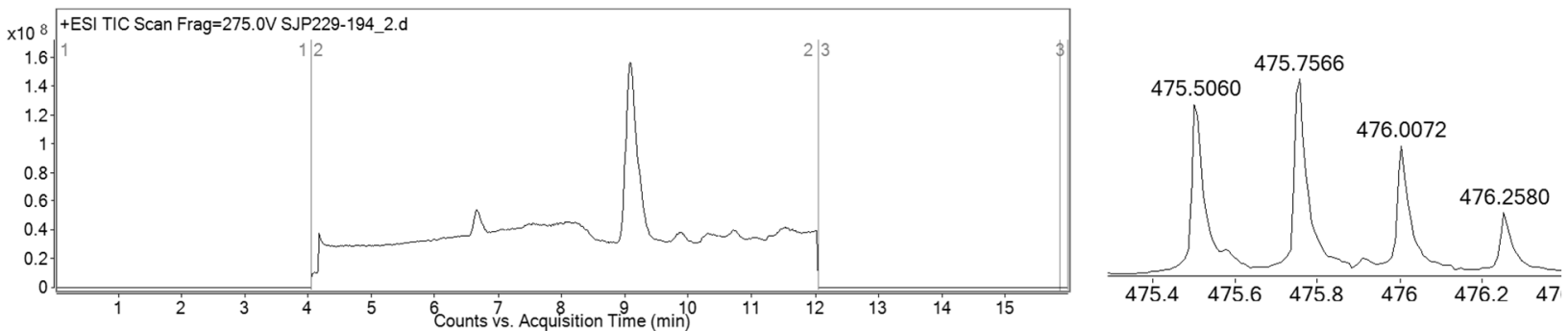

\section{Tetra-GIn-mutant}

Lys - Gln - Gln - Arg - Asp - Gln - Leu - Gln - Ala - Lys - bAla - Lys(Biotin)-CONH 2

HRMS (SI-QToF) $m / z$ : $[\mathrm{M}+4 \mathrm{H}]^{4+}$ calcd. 417.485 found 417.485
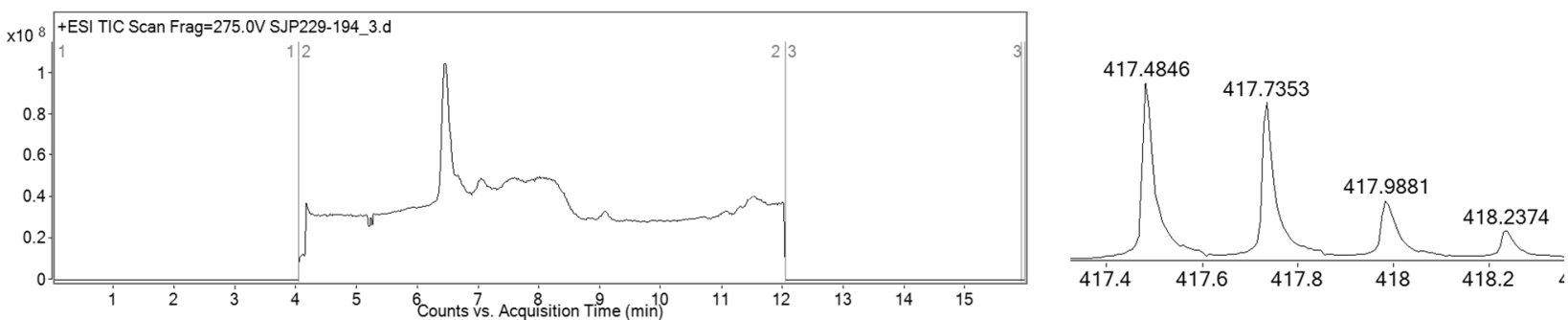
Synthesis and de novo sequencing of three nucleobase peptides test libraries

Procedure for split-and-pool synthesis of three nucleobase peptide test libraries (Alanyl library, homoalanyl library and Dab library): Coupling and Fmoc deprotection steps were performed according to the stoichiometries and procedures described above. Monosized Tentagel beads ( $90 \mu \mathrm{m}$ diameter and loading $0.26 \mathrm{mmol} / \mathrm{g}, 100 \mathrm{mg}$ for each library), were loaded into a peptide synthesis vessel, swollen in DMF and then coupled to Fmoc-Rink Amide linker. For variable positions the resin was suspended in DMF divided in equal aliquots in fritted syringes, coupled to the respective Fmoc amino acid and then pooled back into the peptide synthesis vessel before Fmoc deprotection. After completion of the synthesis the libraries were cleaved according to the general cleavage procedure described above and purified by solid phase extraction.

Sequencing of nucleobase peptide test libraries: Stock solutions of each of the three libraries were prepared $(1 \mathrm{mM}$ in $6 \mathrm{M}$ guanidine $\cdot \mathrm{HCl}, \mathrm{pH}=6.8)$. Prior to injection to the Orbitrap lumos for nanoLC$\mathrm{MS} / \mathrm{MS}$ analysis, the solutions were diluted to $12.8 \mu \mathrm{M}$ solutions in guanidine $\cdot \mathrm{HCl}(1 \mathrm{M}, \mathrm{pH}=6.8) .1 \mu \mathrm{L}$, containing 12.8 pmol library, i.e. $50 \mathrm{fmol}$ per individual member, was injected.

De novo sequencing with PEAKS and further filtering: De novo peptide sequencing was performed in PEAKS 8.5 from Bioinformatics Solutions Inc. (ON, Canada). De novo sequencing was performed allowing $15 \mathrm{ppm}$ assignment errors and $0.05 \mathrm{Da}$ individual fragment mass errors. The nucleobase monomers were selected as variable PTMs (indicated as alanine $+\Delta \mathrm{MS}$ ). 15 candidate sequences were obtained for each preprocessed scan. The complete list of peptide candidates was exported as .csv file and then filtered using a Python script. ${ }^{9}$ Briefly: sequences not matching library design rules (sequence length, correct monomers in every position) were eliminated from further consideration. Next, for scans with multiple remaining sequence candidates, a single peptide with highest ALC (average level of confidence) score per scan was retained, while the rest were excluded. After this, remaining sequences were labeled as "unique" and "non-unique" based on retention time, mass, and sequence similarity criteria. The resulting final list of unique sequences was used for analysis of the sequencing performance of nucleobase peptide libraries containing $0-4$ nucleobase monomers. 
Test libraries for sequencing validation

Library 1 design:

\begin{tabular}{|l|l|l|l|l|l|l|l|c|}
\hline $\mathbf{1}$ & $\mathbf{2}$ & $\mathbf{3}$ & $\mathbf{4}$ & $\mathbf{5}$ & $\mathbf{6}$ & $\mathbf{7}$ & $\mathbf{8}$ & $\mathbf{9}$ \\
\cline { 1 - 7 } Ala & AlaC & Phe & AlaT & AlaG & Trp & AlaA & Tyr & \multirow{2}{*}{ Lys } \\
\cline { 1 - 6 } Ser & His & Leu & Arg & Gln & Pro & Glu & Asn & \\
\hline
\end{tabular}

Full lists of unique sequences for the test library with alanyl nucleobase monomers $U=C$-terminal amide, $B=A l a A, J=A l a C, X=A l a G, Z=A l a T$

\begin{tabular}{|c|c|c|c|c|c|c|}
\hline Peptide & $A L C(\%)$ & $m / z$ & $z$ & $R T$ & Mass & ppm \\
\hline AHFRQPEYKU & 99 & 392.2073 & 3 & 20.23 & 1173.604 & -3.6 \\
\hline SHFRQWENKU & 99 & 410.8744 & 3 & 20.24 & 1229.606 & -3.4 \\
\hline AHFRQWENKU & 99 & 405.5424 & 3 & 20.53 & 1213.61 & -4.2 \\
\hline SJLRQWENKU & 99 & 413.8813 & 3 & 21.03 & 1238.627 & -3.6 \\
\hline AJLRQWENKU & 99 & 408.5499 & 3 & 21.6 & 1222.632 & -3.3 \\
\hline SJFRQWBNKU & 99 & 450.2203 & 3 & 21.84 & 1347.645 & -4.1 \\
\hline AHLZQPBYKU & 99 & 418.8782 & 3 & 22.96 & 1253.617 & -2.9 \\
\hline SJFRQWENKU & 99 & 425.2093 & 3 & 23.39 & 1272.611 & -3.9 \\
\hline AJFRQWENKU & 99 & 419.8784 & 3 & 24.29 & 1256.616 & -2.2 \\
\hline SHLRQWEYKU & 99 & 415.8866 & 3 & 24.55 & 1244.641 & -2.7 \\
\hline SHLZQWBNKU & 99 & 437.5447 & 3 & 24.64 & 1309.618 & -4.2 \\
\hline AHLZQWBNKU & 99 & 432.2132 & 3 & 25.16 & 1293.623 & -4 \\
\hline AHLRQWEYKU & 99 & 410.5546 & 3 & 25.38 & 1228.647 & -3.8 \\
\hline AHFZQPBYKU & 99 & 430.2061 & 3 & 25.75 & 1287.601 & -3.3 \\
\hline SHLZQWENKU & 99 & 412.5336 & 3 & 26.64 & 1234.584 & -4.2 \\
\hline SHFZQWBNKU & 99 & 448.8734 & 3 & 26.87 & 1343.602 & -2.7 \\
\hline AHLZQWENKU & 99 & 407.2019 & 3 & 27.18 & 1218.589 & -4.6 \\
\hline AJLRQWBYKU & 99 & 449.9003 & 3 & 27.52 & 1346.686 & -4.8 \\
\hline AHFZQWBNKU & 99 & 443.5416 & 3 & 27.77 & 1327.607 & -3.1 \\
\hline AHFZQPEYKU & 99 & 405.195 & 3 & 27.94 & 1212.568 & -3.6 \\
\hline SHFRQWEYKU & 99 & 427.2143 & 3 & 28.51 & 1278.626 & -3.7 \\
\hline SJLZQWBNKU & 99 & 451.8797 & 3 & 28.74 & 1352.624 & -4.7 \\
\hline SJLRQWEYKU & 99 & 430.2217 & 3 & 29.22 & 1287.647 & -3 \\
\hline SHFZQWENKU & 99 & 423.8618 & 3 & 29.55 & 1268.569 & -3.9 \\
\hline AHFRQWEYKU & 99 & 421.8823 & 3 & 29.95 & 1262.631 & -4.5 \\
\hline SJFRQWBYKU & 99 & 466.5596 & 3 & 30.31 & 1396.665 & -5.5 \\
\hline AJLRQWEYKU & 99 & 424.8893 & 3 & 30.62 & 1271.652 & -4.8 \\
\hline SJFZQWBNKU & 99 & 463.208 & 3 & 31.38 & 1386.608 & -4.1 \\
\hline SJLZQWENKU & 99 & 639.7998 & 2 & 31.53 & 1277.59 & -3.9 \\
\hline AJLRXWEYKU & 99 & 455.5602 & 3 & 31.6 & 1363.665 & -4.3 \\
\hline AJFRQWBYKU & 99 & 461.228 & 3 & 31.81 & 1380.67 & -5.7 \\
\hline
\end{tabular}




\begin{tabular}{|c|c|c|c|c|c|c|}
\hline AJFZQWBNKU & 99 & 457.8764 & 3 & 32.49 & 1370.613 & -3.9 \\
\hline SJFRQWEYKU & 99 & 441.5495 & 3 & 33.54 & 1321.632 & -3.8 \\
\hline SHLZQWBYKU & 99 & 453.8848 & 3 & 34.86 & 1358.638 & -3.9 \\
\hline SJFZQWENKU & 99 & 656.7924 & 2 & 34.98 & 1311.575 & -3.3 \\
\hline AJFRQWEYKU & 99 & 436.2178 & 3 & 35.32 & 1305.637 & -3.9 \\
\hline AHLZQWBYKU & 99 & 448.5523 & 3 & 35.58 & 1342.643 & -5.9 \\
\hline SHFZQWBYKU & 99 & 465.2137 & 3 & 37.81 & 1392.622 & -2.2 \\
\hline SHLZQWEYKU & 99 & 428.8737 & 3 & 38.51 & 1283.605 & -4.2 \\
\hline AHFZQWBYKU & 99 & 459.8812 & 3 & 38.9 & 1376.627 & -4.2 \\
\hline AHLZQWEYKU & 99 & 423.5423 & 3 & 39.32 & 1267.61 & -3.7 \\
\hline SJLZQWBYKU & 99 & 468.2197 & 3 & 39.44 & 1401.644 & -4.7 \\
\hline AJLZQWBYKU & 99 & 462.8878 & 3 & 40.55 & 1385.649 & -5.3 \\
\hline SHFZQWEYKU & 99 & 440.2021 & 3 & 41.53 & 1317.589 & -3.4 \\
\hline SJFZQWBYKU & 99 & 479.5491 & 3 & 42.37 & 1435.628 & -1.8 \\
\hline AHFZQWEYKU & 99 & 434.8698 & 3 & 42.57 & 1301.594 & -5.2 \\
\hline SJLZQWEYKU & 99 & 664.3096 & 2 & 43.13 & 1326.611 & -4.5 \\
\hline AJFZQWBYKU & 99 & 474.2161 & 3 & 43.73 & 1419.633 & -4.8 \\
\hline AHFZXWEYKU & 99 & 465.5404 & 3 & 44.47 & 1393.606 & -5 \\
\hline SJFZQWEYKU & 99 & 681.303 & 2 & 46.21 & 1360.595 & -2.5 \\
\hline SJFZXWEYKU & 99 & 485.2071 & 3 & 48.21 & 1452.607 & -5.3 \\
\hline AJFZXWEYKU & 99 & 479.8765 & 3 & 49.81 & 1436.612 & -3.2 \\
\hline AJLRQWBNKU & 98 & 433.5605 & 3 & 20.41 & 1297.665 & -4.2 \\
\hline SJFRQPEYKU & 98 & 411.8741 & 3 & 21.48 & 1232.605 & -3.5 \\
\hline SHFRXWENKU & 98 & 441.545 & 3 & 21.5 & 1321.618 & -3.3 \\
\hline SHLRQWBYKU & 98 & 330.9247 & 4 & 23.36 & 1319.675 & -3.8 \\
\hline SJFRXWENKU & 98 & 455.8802 & 3 & 23.93 & 1364.623 & -3.3 \\
\hline AHLRQWBYKU & 98 & 326.9265 & 4 & 24.05 & 1303.68 & -2.5 \\
\hline SHFRQWBYKU & 98 & 339.421 & 4 & 26.36 & 1353.659 & -3.2 \\
\hline AHLRXWEYKU & 98 & 441.2252 & 3 & 27.42 & 1320.659 & -3.7 \\
\hline AHLZXWENKU & 98 & 437.8726 & 3 & 29.07 & 1310.602 & -4.2 \\
\hline AHFZXWBNKU & 98 & 474.2122 & 3 & 31.42 & 1419.619 & -3.3 \\
\hline SJFZQPBNKU & 97 & 433.5331 & 3 & 19.93 & 1297.581 & -3.1 \\
\hline SJLRQWBNKU & 97 & 438.8925 & 3 & 20 & 1313.66 & -3.5 \\
\hline AHLRQWENKU & 97 & 394.2144 & 3 & 20.25 & 1179.626 & -4 \\
\hline SHLRQWENKU & 97 & 399.5461 & 3 & 20.28 & 1195.621 & -3.8 \\
\hline AHFRXWENKU & 97 & 436.2132 & 3 & 21.84 & 1305.623 & -3.9 \\
\hline AJFZQWENKU & 97 & 432.8656 & 3 & 36.22 & 1295.58 & -3.6 \\
\hline AJFRQPEYKU & 96 & 406.5425 & 3 & 22.41 & 1216.61 & -3.6 \\
\hline AJFRQWBNKU & 96 & 333.9187 & 4 & 22.53 & 1331.65 & -3 \\
\hline AHFZQWENKU & 96 & 627.2914 & 2 & 30.32 & 1252.574 & -4.4 \\
\hline SHFZXWBNKU & 96 & 479.5428 & 3 & 30.83 & 1435.614 & -5.3 \\
\hline SHLZQPBYKU & 95 & 424.2096 & 3 & 22.31 & 1269.612 & -3.7 \\
\hline SJLRXWBYKU & 95 & 485.9024 & 3 & 30.22 & 1454.693 & -5.1 \\
\hline AJFZXWBYKU & 95 & 504.8872 & 3 & 47.35 & 1511.646 & -3.8 \\
\hline SHFRQPEYKU & 94 & 397.5391 & 3 & 19.44 & 1189.599 & -3 \\
\hline
\end{tabular}




\begin{tabular}{|c|c|c|c|c|c|c|}
\hline AHFRQWBNKU & 94 & 323.167 & 4 & 20.29 & 1288.644 & -3.7 \\
\hline SJFRXWBYKU & 94 & 497.2317 & 3 & 34.13 & 1488.677 & -2.7 \\
\hline AJLZXWENKU & 94 & 452.2083 & 3 & 34.24 & 1353.607 & -3.3 \\
\hline SJLRQPEYKU & 93 & 400.5462 & 3 & 19.27 & 1198.621 & -3.2 \\
\hline AHFRQWBYKU & 93 & 335.4222 & 4 & 27.91 & 1337.664 & -3.1 \\
\hline AJFZQPEYKU & 93 & 419.5298 & 3 & 31.28 & 1255.574 & -4.7 \\
\hline AJLRQPBYKU & 92 & 420.2255 & 3 & 19.68 & 1257.659 & -3.5 \\
\hline SHFRQWBNKU & 92 & 435.8854 & 3 & 20.15 & 1304.639 & -3.2 \\
\hline SHFRXWEYKU & 92 & 457.8847 & 3 & 30.65 & 1370.638 & -4.2 \\
\hline SJLZQPBNKU & 91 & 422.205 & 3 & 18.5 & 1263.597 & -3 \\
\hline AHFRQPBYKU & 91 & 417.2186 & 3 & 19.91 & 1248.638 & -3.1 \\
\hline AJLRXWBYKU & 91 & 480.5711 & 3 & 31.21 & 1438.698 & -4.4 \\
\hline AJLZQWENKU & 91 & 421.5368 & 3 & 32.63 & 1261.595 & -5.2 \\
\hline AJLZXWBNKU & 91 & 477.219 & 3 & 33.53 & 1428.641 & -4 \\
\hline AJFZXWBNKU & 91 & 488.5471 & 3 & 37.26 & 1462.625 & -3.7 \\
\hline AHFZXWBYKU & 91 & 490.5526 & 3 & 42.25 & 1468.64 & -2.4 \\
\hline SJLRQWBYKU & 90 & 455.2324 & 3 & 26.23 & 1362.681 & -3.9 \\
\hline SHFRXWBYKU & 89 & 362.4234 & 4 & 30.09 & 1445.671 & -4.7 \\
\hline SJLZXWEYKU & 89 & 473.8788 & 3 & 44.8 & 1418.623 & -5.8 \\
\hline AJLZXWEYKU & 89 & 468.5476 & 3 & 46.29 & 1402.628 & -4.9 \\
\hline AHLZQPEYKU & 88 & 393.8673 & 3 & 24.38 & 1178.583 & -2.7 \\
\hline AJFRXWENKU & 88 & 450.5482 & 3 & 24.77 & 1348.628 & -4.1 \\
\hline SHFZXWENKU & 88 & 454.5331 & 3 & 32.15 & 1360.581 & -2.6 \\
\hline SHFZQPENKU & 87 & 394.1866 & 3 & 18.3 & 1179.542 & -3.7 \\
\hline SJFZQPENKU & 87 & 612.2793 & 2 & 20.25 & 1222.548 & -3.1 \\
\hline SHFRXWBNKU & 87 & 350.1688 & 4 & 21.74 & 1396.651 & -3.4 \\
\hline SJFZQPBYKU & 87 & 449.8728 & 3 & 28.18 & 1346.602 & -3.8 \\
\hline AHFZXWENKU & 87 & 449.2008 & 3 & 32.61 & 1344.586 & -4 \\
\hline AHLZXWEYKU & 87 & 454.212 & 3 & 40.39 & 1359.622 & -5.7 \\
\hline AJLZQPBYKU & 86 & 433.2135 & 3 & 25.88 & 1296.622 & -2.9 \\
\hline SJFRQPBYKU & 85 & 436.885 & 3 & 20.75 & 1307.638 & -4 \\
\hline AHFRXWEYKU & 85 & 452.5527 & 3 & 32.04 & 1354.643 & -5.1 \\
\hline AJFRXWBNKU & 84 & 475.5592 & 3 & 24.76 & 1423.662 & -4.2 \\
\hline SHLZXWBNKU & 83 & 468.2159 & 3 & 28.33 & 1401.63 & -3 \\
\hline SHLRXWEYKU & 82 & 335.1698 & 4 & 26.89 & 1336.654 & -2.7 \\
\hline SJLRXWEYKU & 82 & 460.8914 & 3 & 30.43 & 1379.659 & -5.1 \\
\hline AJLZQPBNKU & 81 & 416.8732 & 3 & 18.83 & 1247.602 & -3.4 \\
\hline SJFZXWENKU & 81 & 468.8677 & 3 & 37.11 & 1403.587 & -3.8 \\
\hline AHLRXWBYKU & 80 & 349.9289 & 4 & 27.35 & 1395.692 & -4.1 \\
\hline AHLZXWBYKU & 80 & 479.2242 & 3 & 39.99 & 1434.655 & -3.1 \\
\hline AHFZQPBNKU & 79 & 413.8662 & 3 & 18.74 & 1238.581 & -3 \\
\hline AHFZQPENKU & 78 & 582.7794 & 2 & 18.6 & 1163.547 & -2.4 \\
\hline SHFZQPEYKU & 78 & 615.2871 & 2 & 26.94 & 1228.563 & -2.3 \\
\hline SJLRQPBYKU & 77 & 425.5573 & 3 & 19.31 & 1273.654 & -3 \\
\hline SHFRQPBYKU & 77 & 317.1642 & 4 & 19.49 & 1264.633 & -3.7 \\
\hline
\end{tabular}




\begin{tabular}{|c|c|c|c|c|c|c|}
\hline SJLZXWBNKU & 77 & 482.5512 & 3 & 32.75 & 1444.636 & -2.7 \\
\hline AJFRXWBYKU & 77 & 491.8991 & 3 & 35.27 & 1472.682 & -4.4 \\
\hline AHFRXWBNKU & 76 & 346.1701 & 4 & 22.11 & 1380.656 & -3.5 \\
\hline AHFRXWBYKU & 76 & 358.4248 & 4 & 30.92 & 1429.677 & -4.6 \\
\hline AJLZQWEYKU & 73 & 437.8772 & 3 & 44.23 & 1310.616 & -4.6 \\
\hline AHLRXPEYKU & 72 & 411.55 & 3 & 19.23 & 1231.632 & -3.1 \\
\hline SHFZXWEYKU & 72 & 470.8728 & 3 & 43.37 & 1409.601 & -3.4 \\
\hline AHLZXWBNKU & 71 & 462.8836 & 3 & 28.82 & 1385.635 & -4.4 \\
\hline SJFZXWBYKU & 71 & 510.219 & 3 & 45.95 & 1527.64 & -3.5 \\
\hline SHLZQPEYKU & 70 & 399.1988 & 3 & 23.75 & 1194.578 & -3 \\
\hline SHLZXWEYKU & 70 & 459.5446 & 3 & 40.68 & 1375.617 & -3.7 \\
\hline SHLZXWBYKU & 69 & 484.555 & 3 & 39.05 & 1450.65 & -4.9 \\
\hline AJFZQWEYKU & 69 & 449.205 & 3 & 47.58 & 1344.6 & -5.1 \\
\hline AJFZQPBNKU & 66 & 428.2007 & 3 & 20.5 & 1281.586 & -4.8 \\
\hline AJFRQPBYKU & 64 & 431.5538 & 3 & 21.17 & 1291.643 & -3 \\
\hline AJLZQPENKU & 63 & 587.2904 & 2 & 18.66 & 1172.569 & -2 \\
\hline AJFZXWENKU & 63 & 463.5359 & 3 & 38.39 & 1387.592 & -4.4 \\
\hline SJFRXWEYKU & 61 & 472.2193 & 3 & 35.13 & 1413.644 & -5.5 \\
\hline SHFRQPENKU & 59 & 571.2955 & 2 & 56.72 & 1140.579 & -2.2 \\
\hline AJLRXWENKU & 58 & 439.2203 & 3 & 21.98 & 1314.644 & -3.8 \\
\hline SJLZQPENKU & 53 & 595.2874 & 2 & 18.26 & 1188.564 & -2.8 \\
\hline
\end{tabular}


Library 2 design:

\begin{tabular}{|l|l|l|l|l|l|l|l|c|}
\hline $\mathbf{1}$ & $\mathbf{2}$ & $\mathbf{3}$ & $\mathbf{4}$ & $\mathbf{5}$ & $\mathbf{6}$ & $\mathbf{7}$ & $\mathbf{8}$ & $\mathbf{9}$ \\
\cline { 1 - 7 } Ala & hAlaC & Phe & hAlaT & hAlaG & Trp & hAlaA & Tyr & \multirow{2}{*}{ Lys } \\
\hline Ser & His & Leu & Arg & Gln & Pro & Glu & Asn & \\
\hline
\end{tabular}

Full lists of unique sequences for the test library with homoalanyl nucleobase monomers $U=C$-terminal amide, $B=h A l a A, J=h A l a C, X=h A l a G, Z=h A l a T$

\begin{tabular}{|c|c|c|c|c|c|c|}
\hline Peptide & $A L C(\%)$ & $m / z$ & $z$ & $R T$ & Mass & ppm \\
\hline AHFZQPBNKU & 99 & 423.2116 & 3 & 18.02 & 1266.605 & 6.7 \\
\hline SHFRQPEYKU & 99 & 397.5396 & 3 & 18.21 & 1189.599 & -1.9 \\
\hline SHFZQPENKU & 99 & 398.8591 & 3 & 18.27 & 1193.555 & 0.5 \\
\hline SHFRQWENKU & 99 & 410.8746 & 3 & 18.8 & 1229.606 & -2.8 \\
\hline AHFRQPEYKU & 99 & 392.2077 & 3 & 19.08 & 1173.604 & -2.5 \\
\hline AHFRQWENKU & 99 & 405.5429 & 3 & 19.13 & 1213.61 & -2.9 \\
\hline AHLZXPENKU & 99 & 417.5414 & 3 & 19.6 & 1249.604 & -1.3 \\
\hline SJLRQWENKU & 99 & 418.5536 & 3 & 20.31 & 1252.639 & 0 \\
\hline AHFRXWENKU & 99 & 440.8849 & 3 & 21 & 1319.639 & -4.5 \\
\hline AHLZQPBYKU & 99 & 428.2235 & 3 & 22.78 & 1281.64 & 6.4 \\
\hline SHLZQPEYKU & 99 & 403.8709 & 3 & 23.71 & 1208.591 & 0 \\
\hline SHFZQPBYKU & 99 & 444.8817 & 3 & 23.9 & 1331.62 & 2.6 \\
\hline SHLRQWEYKU & 99 & 415.886 & 3 & 24.11 & 1244.641 & -4.1 \\
\hline AHFZQPBYKU & 99 & 439.55 & 3 & 24.71 & 1315.625 & 2.6 \\
\hline AHLRQWEYKU & 99 & 410.5535 & 3 & 25.12 & 1228.647 & -6.4 \\
\hline AHLRXWEYKU & 99 & 445.8977 & 3 & 26.57 & 1334.675 & -2.9 \\
\hline SHLZXPEYKU & 99 & 439.2134 & 3 & 27.02 & 1314.619 & -0.7 \\
\hline AHFRQWBYKU & 99 & 451.5661 & 3 & 27.13 & 1351.675 & 0.8 \\
\hline AHLZQWBNKU & 99 & 441.556 & 3 & 27.53 & 1321.647 & -0.4 \\
\hline SJLRQWEYKU & 99 & 434.8938 & 3 & 27.62 & 1301.66 & 0 \\
\hline AHLZQWENKU & 99 & 411.8748 & 3 & 27.72 & 1232.602 & 0.3 \\
\hline SHLZQWBNKU & 99 & 446.8889 & 3 & 28.01 & 1337.642 & 2.3 \\
\hline AHLZXWBNKU & 99 & 476.8998 & 3 & 28.05 & 1427.675 & 1.7 \\
\hline AHLZXPEYKU & 99 & 433.8809 & 3 & 28.12 & 1298.625 & -2.8 \\
\hline SHFRQWEYKU & 99 & 427.2149 & 3 & 28.25 & 1278.626 & -2.2 \\
\hline AHFZQPEYKU & 99 & 409.8671 & 3 & 28.49 & 1226.58 & -0.6 \\
\hline SHLZXWENKU & 99 & 452.5478 & 3 & 29.44 & 1354.626 & -2.9 \\
\hline AHLZXWENKU & 99 & 447.2163 & 3 & 29.97 & 1338.631 & -2.6 \\
\hline AHFRQWEYKU & 99 & 421.8824 & 3 & 30.09 & 1262.631 & -4.2 \\
\hline AHFZQWBNKU & 99 & 452.886 & 3 & 31.04 & 1355.631 & 4 \\
\hline SHFZQWBNKU & 99 & 458.2152 & 3 & 31.25 & 1371.626 & -1.5 \\
\hline SHFZQWENKU & 99 & 428.5341 & 3 & 31.39 & 1282.581 & -0.7 \\
\hline AHFZQWENKU & 99 & 423.2029 & 3 & 32.14 & 1266.586 & 0.5 \\
\hline SJFRQWEYKU & 99 & 446.2207 & 3 & 32.47 & 1335.644 & -2.9 \\
\hline AHFZXPEYKU & 99 & 445.2097 & 3 & 32.79 & 1332.609 & -1.2 \\
\hline SHFZXWENKU & 99 & 463.8763 & 3 & 32.95 & 1388.61 & -2 \\
\hline
\end{tabular}




\begin{tabular}{|c|c|c|c|c|c|c|}
\hline AHFZXWENKU & 99 & 458.5442 & 3 & 33.78 & 1372.615 & -3 \\
\hline SHLZQWBYKU & 99 & 463.2287 & 3 & 36.6 & 1386.662 & 1.7 \\
\hline AHLZQWBYKU & 99 & 457.8978 & 3 & 37.04 & 1370.667 & 3.4 \\
\hline AHLZXWBYKU & 99 & 493.2404 & 3 & 38.47 & 1476.696 & 2.6 \\
\hline SHFZQWBYKU & 99 & 474.5564 & 3 & 39.7 & 1420.646 & 0.9 \\
\hline AHFZQWBYKU & 99 & 469.2244 & 3 & 40.58 & 1404.651 & 0 \\
\hline AHLZQWEYKU & 99 & 428.2141 & 3 & 40.71 & 1281.623 & -1.6 \\
\hline AHLZXWEYKU & 99 & 463.5563 & 3 & 41.15 & 1387.651 & -2.9 \\
\hline AHFZXWBYKU & 99 & 378.6775 & 4 & 41.81 & 1510.68 & 0.6 \\
\hline SHFZQWEYKU & 99 & 666.8074 & 2 & 43.82 & 1331.602 & -1.1 \\
\hline SHFZXWEYKU & 99 & 480.216 & 3 & 44.06 & 1437.63 & -3 \\
\hline AHFZQWEYKU & 99 & 439.5416 & 3 & 45.12 & 1315.607 & -3 \\
\hline AHFZXWEYKU & 99 & 474.8837 & 3 & 45.19 & 1421.636 & -4.4 \\
\hline SJFZXWEYKU & 99 & 499.2241 & 3 & 47.24 & 1494.648 & 1.3 \\
\hline SHFZQPBNKU & 98 & 428.5418 & 3 & 17.65 & 1282.599 & 3.4 \\
\hline SJLRQPEYKU & 98 & 405.2179 & 3 & 18.14 & 1212.633 & -0.9 \\
\hline AHLRXWENKU & 98 & 429.5605 & 3 & 18.71 & 1285.655 & 4 \\
\hline AHFZQPENKU & 98 & 393.5273 & 3 & 18.77 & 1177.56 & 0.3 \\
\hline SJFRQPBYKU & 98 & 446.2294 & 3 & 18.79 & 1335.662 & 3.3 \\
\hline SHLZXPENKU & 98 & 422.873 & 3 & 18.95 & 1265.599 & -1.4 \\
\hline AHFRQWBNKU & 98 & 326.6713 & 4 & 20.15 & 1302.655 & 0.9 \\
\hline AHFZXPBNKU & 98 & 458.5526 & 3 & 21.25 & 1372.633 & 2.1 \\
\hline SHLZQPBYKU & 98 & 433.5542 & 3 & 22.25 & 1297.636 & 4.1 \\
\hline SJFRQWENKU & 98 & 429.8812 & 3 & 22.58 & 1286.624 & -1.4 \\
\hline SHLRQWBYKU & 98 & 334.4287 & 4 & 23.49 & 1333.686 & -0.3 \\
\hline AHLZQPEYKU & 98 & 398.5392 & 3 & 24.58 & 1192.596 & 0 \\
\hline SHFZQPEYKU & 98 & 415.1993 & 3 & 27.36 & 1242.575 & 0.8 \\
\hline SHFRXWEYKU & 98 & 347.169 & 4 & 29.89 & 1384.654 & -5.4 \\
\hline SJLZXPEYKU & 98 & 458.219 & 3 & 30.29 & 1371.638 & -1.8 \\
\hline SJFZQPEYKU & 98 & 434.2049 & 3 & 31.06 & 1299.594 & -0.4 \\
\hline SJLZXWENKU & 98 & 471.5559 & 3 & 35.47 & 1411.644 & 1.4 \\
\hline SHLZXWBYKU & 98 & 498.5714 & 3 & 38.05 & 1492.69 & 1.3 \\
\hline SJFZQWBNKU & 98 & 477.224 & 3 & 38.21 & 1428.644 & 4.3 \\
\hline SJFZXWENKU & 98 & 482.8838 & 3 & 38.43 & 1445.628 & 0.9 \\
\hline SJLZQWBYKU & 98 & 482.2351 & 3 & 42.41 & 1443.68 & 2.2 \\
\hline SJFZQWBYKU & 98 & 493.5644 & 3 & 44.44 & 1477.665 & 4.7 \\
\hline SHLRXWENKU & 97 & 326.4186 & 4 & 18.45 & 1301.65 & -3.3 \\
\hline AJFRQPBYKU & 97 & 440.8978 & 3 & 18.83 & 1319.667 & 3.4 \\
\hline SJFZQPBNKU & 97 & 447.5486 & 3 & 19.74 & 1339.617 & 5 \\
\hline SHFRQWBNKU & 97 & 330.6698 & 4 & 19.98 & 1318.65 & 0.1 \\
\hline SJFZQPENKU & 97 & 417.8656 & 3 & 20.98 & 1250.573 & 1.7 \\
\hline AJFZQPENKU & 97 & 412.5341 & 3 & 21.12 & 1234.578 & 1.9 \\
\hline SHFZXPENKU & 97 & 434.2004 & 3 & 21.89 & 1299.583 & -3 \\
\hline SJFRQWBNKU & 97 & 459.5647 & 3 & 23.4 & 1375.668 & 2.9 \\
\hline AHLRQWBYKU & 97 & 330.4298 & 4 & 24.03 & 1317.691 & -0.7 \\
\hline
\end{tabular}




\begin{tabular}{|c|c|c|c|c|c|c|}
\hline SJLZQPBYKU & 97 & 452.56 & 3 & 24.75 & 1354.654 & 3.5 \\
\hline AHLRXWBYKU & 97 & 356.9363 & 4 & 25.59 & 1423.72 & -2.4 \\
\hline SHLRXWEYKU & 97 & 338.6731 & 4 & 26.12 & 1350.67 & -4.9 \\
\hline SJLZQPEYKU & 97 & 422.8782 & 3 & 26.84 & 1265.609 & 2.9 \\
\hline SJFZQPBYKU & 97 & 463.889 & 3 & 27.2 & 1388.638 & 5.2 \\
\hline SJFRQWBYKU & 97 & 357.1793 & 4 & 30.5 & 1424.689 & -0.3 \\
\hline AHFZXWBNKU & 97 & 488.2277 & 3 & 31.78 & 1461.659 & 1.2 \\
\hline AJFRXWEYKU & 97 & 476.2319 & 3 & 33.28 & 1425.678 & -2.5 \\
\hline SJFZXPEYKU & 97 & 469.5474 & 3 & 34.61 & 1405.622 & -1 \\
\hline AJFZXWEYKU & 97 & 493.8935 & 3 & 47.71 & 1478.654 & 3.4 \\
\hline SHLRQWBNKU & 96 & 322.174 & 4 & 18.42 & 1284.666 & 0.9 \\
\hline AJLRXWENKU & 96 & 448.5642 & 3 & 20.56 & 1342.673 & -1.5 \\
\hline SJLZXPENKU & 96 & 441.8802 & 3 & 21.46 & 1322.617 & 1.3 \\
\hline SJLRXWENKU & 96 & 453.8947 & 3 & 21.66 & 1358.668 & -4 \\
\hline SJLRQWBNKU & 96 & 336.4283 & 4 & 21.69 & 1341.684 & 0.2 \\
\hline AHFRXPEYKU & 96 & 427.5495 & 3 & 21.92 & 1279.633 & -4.9 \\
\hline SHFRQWBYKU & 96 & 342.9247 & 4 & 26.34 & 1367.67 & -0.6 \\
\hline AJLZXWEYKU & 96 & 482.5634 & 3 & 43.5 & 1444.669 & -0.5 \\
\hline SJFZQWEYKU & 96 & 463.8809 & 3 & 47.05 & 1388.62 & 0.6 \\
\hline SJLRQPBYKU & 95 & 434.9009 & 3 & 17.82 & 1301.678 & 2.6 \\
\hline AJLZQPENKU & 95 & 401.2056 & 3 & 18.3 & 1200.594 & 1.1 \\
\hline AHLRXPEYKU & 95 & 416.2214 & 3 & 19.03 & 1245.648 & -4.8 \\
\hline AHLZXPBNKU & 95 & 447.2239 & 3 & 19.38 & 1338.649 & 0.8 \\
\hline AJFRQPEYKU & 95 & 411.2141 & 3 & 20.85 & 1230.623 & -1.7 \\
\hline AJLZQPEYKU & 95 & 417.5464 & 3 & 27.03 & 1249.614 & 2.4 \\
\hline AHFRXWBYKU & 95 & 365.4332 & 4 & 29.05 & 1457.704 & -0.2 \\
\hline AJFZQPEYKU & 95 & 428.8728 & 3 & 31.66 & 1283.598 & -1.3 \\
\hline SJLZQWENKU & 95 & 436.2126 & 3 & 34.22 & 1305.615 & 0.7 \\
\hline SJLZQWBNKU & 95 & 465.896 & 3 & 36.91 & 1394.66 & 4.6 \\
\hline AHLRXWBNKU & 94 & 344.6818 & 4 & 19.06 & 1374.699 & -0.9 \\
\hline SJFRQPEYKU & 94 & 416.5466 & 3 & 20.9 & 1246.617 & 0.5 \\
\hline AJFZXPENKU & 94 & 447.8758 & 3 & 25.71 & 1340.607 & -0.8 \\
\hline SJLZQPBNKU & 93 & 436.2207 & 3 & 18.44 & 1305.633 & 5.4 \\
\hline AJLRQWENKU & 93 & 413.222 & 3 & 18.94 & 1236.644 & -0.1 \\
\hline SJFRXWENKU & 93 & 465.2241 & 3 & 23.85 & 1392.652 & -1.2 \\
\hline SHLRXWBYKU & 93 & 360.9349 & 4 & 25.16 & 1439.715 & -2.9 \\
\hline SJFZXPENKU & 93 & 453.2068 & 3 & 25.4 & 1356.602 & -2.1 \\
\hline AJLZXPEYKU & 93 & 452.8888 & 3 & 30.75 & 1355.643 & 1.5 \\
\hline SJLZQWEYKU & 93 & 452.5518 & 3 & 43.72 & 1354.636 & -1.7 \\
\hline SHLRXPEYKU & 92 & 316.4172 & 4 & 18.45 & 1261.643 & -2.7 \\
\hline SHFZXPBNKU & 92 & 348.1648 & 4 & 20.59 & 1388.628 & 1.5 \\
\hline AHFRXWBNKU & 92 & 353.1781 & 4 & 21.49 & 1408.684 & -0.3 \\
\hline AHLZXPBYKU & 92 & 347.925 & 4 & 26.44 & 1387.669 & 1.5 \\
\hline SJFZXWBYKU & 92 & 396.9316 & 4 & 44.83 & 1583.693 & 2.7 \\
\hline AJFZQPBNKU & 91 & 442.217 & 3 & 19.84 & 1323.623 & 5 \\
\hline
\end{tabular}




\begin{tabular}{|c|c|c|c|c|c|c|}
\hline SJLRXWEYKU & 91 & 470.2364 & 3 & 28.87 & 1407.688 & -0.7 \\
\hline SHFZXWBNKU & 91 & 493.5585 & 3 & 31.1 & 1477.655 & -0.6 \\
\hline AJFZQWENKU & 91 & 442.2094 & 3 & 36.82 & 1323.605 & 1.3 \\
\hline SHFZXWBYKU & 91 & 382.6757 & 4 & 41.07 & 1526.675 & -0.8 \\
\hline SHLRXWBNKU & 90 & 348.6808 & 4 & 18.83 & 1390.694 & -0.1 \\
\hline SHLZXPBNKU & 90 & 339.6689 & 4 & 18.91 & 1354.644 & 2 \\
\hline SHFRXWENKU & 90 & 334.9145 & 4 & 20.6 & 1335.634 & -3.8 \\
\hline SJLZXPBNKU & 90 & 471.5635 & 3 & 20.88 & 1411.662 & 4.9 \\
\hline AJFZQPBYKU & 90 & 458.5573 & 3 & 27.44 & 1372.643 & 5.1 \\
\hline SJFZXPBYKU & 90 & 374.6754 & 4 & 31.9 & 1494.667 & 4.1 \\
\hline AJLZXWENKU & 90 & 466.2236 & 3 & 33.98 & 1395.649 & 0 \\
\hline AJLZQPBNKU & 89 & 430.8893 & 3 & 18.4 & 1289.638 & 6.1 \\
\hline AJFRXWBNKU & 89 & 489.5753 & 3 & 23.38 & 1465.702 & 1.7 \\
\hline SJFRXPEYKU & 89 & 451.889 & 3 & 23.76 & 1352.646 & -0.7 \\
\hline AJFRQWBYKU & 89 & 353.1815 & 4 & 30.61 & 1408.694 & 2.4 \\
\hline SHLZXWEYKU & 89 & 702.8286 & 2 & 40.95 & 1403.646 & -2.5 \\
\hline SJLRXWBNKU & 88 & 362.9362 & 4 & 21.82 & 1447.712 & 2.4 \\
\hline AJLZQWBNKU & 88 & 460.5645 & 3 & 32.03 & 1378.665 & 5 \\
\hline AJFZXPEYKU & 88 & 464.2175 & 3 & 35.31 & 1389.627 & 2.6 \\
\hline SJLZXWBNKU & 88 & 501.239 & 3 & 35.4 & 1500.688 & 4.7 \\
\hline AJLZQWBYKU & 88 & 476.9041 & 3 & 40.14 & 1427.685 & 3.7 \\
\hline SJLZXWBYKU & 88 & 388.4347 & 4 & 42.6 & 1549.709 & 0.6 \\
\hline AJFZQWBYKU & 88 & 488.2313 & 3 & 44.03 & 1461.67 & 1.7 \\
\hline SJLZXWEYKU & 88 & 731.3401 & 2 & 44.24 & 1460.664 & 1.2 \\
\hline AJLRQWBNKU & 87 & 332.4305 & 4 & 20.19 & 1325.689 & 3 \\
\hline SJFZXPBNKU & 87 & 482.8906 & 3 & 23.21 & 1445.646 & 2.7 \\
\hline AJFZXPBNKU & 87 & 477.5592 & 3 & 23.53 & 1429.651 & 3.3 \\
\hline AJLZQPBYKU & 87 & 447.2281 & 3 & 24.98 & 1338.659 & 2.7 \\
\hline AJLRQWEYKU & 87 & 429.5619 & 3 & 27.41 & 1285.665 & -0.7 \\
\hline AJFZXWBNKU & 87 & 507.2353 & 3 & 36.08 & 1518.678 & 4.2 \\
\hline AJFZXWBYKU & 87 & 523.5753 & 3 & 44.77 & 1567.698 & 3.9 \\
\hline AJFRXPBYKU & 86 & 476.2392 & 3 & 22.43 & 1425.696 & 0.1 \\
\hline AJFRQWBNKU & 86 & 340.9267 & 4 & 22.83 & 1359.673 & 3.5 \\
\hline AJFRXPEYKU & 86 & 446.5569 & 3 & 24.41 & 1336.651 & -1.8 \\
\hline AHFRXWEYKU & 86 & 343.1701 & 4 & 31.18 & 1368.659 & -5.9 \\
\hline AJFRQWEYKU & 86 & 440.8906 & 3 & 32.74 & 1319.649 & 0.6 \\
\hline AJLZQWEYKU & 86 & 670.3287 & 2 & 43.33 & 1338.641 & 1.7 \\
\hline AJLZQWENKU & 85 & 645.8191 & 2 & 31.9 & 1289.62 & 2.5 \\
\hline AJFZXWENKU & 85 & 715.824 & 2 & 38.61 & 1429.633 & 0.2 \\
\hline SHLZQWENKU & 84 & 625.3054 & 2 & 27.72 & 1248.597 & -0.6 \\
\hline SHLRQPEYKU & 83 & 386.2111 & 3 & 17.2 & 1155.615 & -2.9 \\
\hline SHFRXPBYKU & 83 & 347.1753 & 4 & 19.64 & 1384.672 & -0.3 \\
\hline SHFRXPEYKU & 83 & 324.9131 & 4 & 21.24 & 1295.628 & -3.5 \\
\hline AJFZQWBNKU & 83 & 707.3346 & 2 & 35.87 & 1412.649 & 3.9 \\
\hline SJLRQWBYKU & 82 & 348.6842 & 4 & 27 & 1390.704 & 2.5 \\
\hline
\end{tabular}




\begin{tabular}{|c|c|c|c|c|c|c|}
\hline AJLZXPENKU & 81 & 436.5486 & 3 & 21.74 & 1306.622 & 1.4 \\
\hline AJLRQPEYKU & 80 & 399.886 & 3 & 18.25 & 1196.638 & -1.7 \\
\hline AJLRXWBNKU & 80 & 358.9366 & 4 & 20.71 & 1431.717 & 0 \\
\hline AJFRQWENKU & 80 & 424.5495 & 3 & 22.07 & 1270.629 & -1.5 \\
\hline AJLRQWBYKU & 79 & 344.6858 & 4 & 26.63 & 1374.709 & 3.4 \\
\hline AJLRXPEYKU & 78 & 435.2291 & 3 & 21.23 & 1302.667 & -1 \\
\hline SJFZQWENKU & 77 & 447.5406 & 3 & 37.2 & 1339.6 & 0.2 \\
\hline AJLZXWBYKU & 77 & 512.246 & 3 & 41.17 & 1533.714 & 1.6 \\
\hline SJLRXPBYKU & 76 & 470.2426 & 3 & 20.07 & 1407.706 & -0.2 \\
\hline SHLZXWBNKU & 76 & 361.925 & 4 & 27.83 & 1443.67 & 0.6 \\
\hline AJFZXPBYKU & 76 & 493.8997 & 3 & 32.82 & 1478.672 & 3.9 \\
\hline AJLZXPBNKU & 75 & 466.2315 & 3 & 21.24 & 1395.667 & 4.3 \\
\hline AJLZXPBYKU & 75 & 362.1805 & 4 & 29.12 & 1444.687 & 3.9 \\
\hline SHFZXPBYKU & 74 & 360.4199 & 4 & 28.52 & 1437.648 & 1.5 \\
\hline AJFZQWEYKU & 74 & 458.5493 & 3 & 47.53 & 1372.625 & 0.8 \\
\hline AJLRXWEYKU & 72 & 464.9033 & 3 & 28.15 & 1391.693 & -3.8 \\
\hline AHFZXPBYKU & 72 & 474.8919 & 3 & 29.71 & 1421.653 & 0.3 \\
\hline SHFZXPEYKU & 72 & 450.5412 & 3 & 31.36 & 1348.604 & -1.5 \\
\hline AJLZXWBNKU & 71 & 495.9073 & 3 & 32.07 & 1484.693 & 4.4 \\
\hline SHLRXPBYKU & 70 & 338.679 & 4 & 18.15 & 1350.688 & -0.7 \\
\hline AJLRXWBYKU & 70 & 494.5876 & 3 & 27.07 & 1480.738 & 2.1 \\
\hline AHLRQPBYKU & 69 & 410.5625 & 3 & 17.25 & 1228.665 & 0.8 \\
\hline AHLRXPBYKU & 69 & 334.6808 & 4 & 18.62 & 1334.693 & 0.6 \\
\hline SHLZXPBYKU & 68 & 351.9233 & 4 & 25.77 & 1403.664 & 0.1 \\
\hline SJFRXPBYKU & 67 & 361.4298 & 4 & 22.37 & 1441.69 & -0.3 \\
\hline SHLZQWEYKU & 64 & 433.5445 & 3 & 39.46 & 1297.617 & -4.5 \\
\hline AJLRXPBYKU & 61 & 348.9359 & 4 & 20.2 & 1391.711 & 2.5 \\
\hline AHFRXPBYKU & 60 & 343.1765 & 4 & 20.42 & 1368.677 & -0.4 \\
\hline SJFZXWBNKU & 60 & 512.5652 & 3 & 37.3 & 1534.673 & 0.9 \\
\hline AHLRQPEYKU & 57 & 380.8793 & 3 & 17.42 & 1139.62 & -3.4 \\
\hline AHFRQPBYKU & 57 & 421.8904 & 3 & 17.63 & 1262.649 & 0.5 \\
\hline SHLRQPBYKU & 55 & 415.8936 & 3 & 26.05 & 1244.659 & -0.3 \\
\hline
\end{tabular}


Library 3 design:

\begin{tabular}{|l|l|l|l|l|l|l|l|c|}
\hline $\mathbf{1}$ & $\mathbf{2}$ & $\mathbf{3}$ & $\mathbf{4}$ & $\mathbf{5}$ & $\mathbf{6}$ & $\mathbf{7}$ & $\mathbf{8}$ & $\mathbf{9}$ \\
\cline { 1 - 7 } Ala & DabC & Phe & DabT & DabG & Trp & DabA & Tyr & \multirow{2}{*}{ Lys } \\
\cline { 1 - 5 } Ser & His & Leu & Arg & Gln & Pro & Glu & Asn & \\
\hline
\end{tabular}

Full lists of unique sequences for the test library with diaminobutanoic acid based nucleobase monomers

$U=C$-terminal amide, $B=D a b A, J=D a b C, X=D a b G, Z=D a b T$

\begin{tabular}{|c|c|c|c|c|c|c|}
\hline Peptide & $A L C(\%)$ & $m / z$ & $z$ & $R T$ & Mass & ppm \\
\hline SHFZQPENKU & 99 & 417.8659 & 3 & 15.62 & 1250.575 & 1 \\
\hline SHLZZPENKU & 99 & 460.8875 & 3 & 15.84 & 1379.639 & 1.4 \\
\hline SHFRQPEYKU & 99 & 397.5388 & 3 & 15.94 & 1189.599 & -3.8 \\
\hline AHLRZPEYKU & 99 & 435.2289 & 3 & 16.02 & 1302.669 & -2.7 \\
\hline AHLZZPENKU & 99 & 455.5548 & 3 & 16.63 & 1363.644 & -1.1 \\
\hline AHFRQPEYKU & 99 & 392.2072 & 3 & 16.66 & 1173.604 & -3.8 \\
\hline SHFRQWENKU & 99 & 410.8743 & 3 & 16.82 & 1229.606 & -3.6 \\
\hline SHLRZWENKU & 99 & 453.8949 & 3 & 16.92 & 1358.67 & -5.1 \\
\hline AHFRQWENKU & 99 & 405.5417 & 3 & 17.09 & 1213.61 & -5.8 \\
\hline AHFRQWZNKU & 99 & 454.2322 & 3 & 17.88 & 1359.675 & -0.1 \\
\hline SHFZZPENKU & 99 & 472.2149 & 3 & 18.01 & 1413.623 & -0.4 \\
\hline AHFZZPENKU & 99 & 466.8833 & 3 & 18.96 & 1397.628 & -0.2 \\
\hline SHFRZWZNKU & 99 & 513.9144 & 3 & 19.65 & 1538.719 & 1.8 \\
\hline SHFRZWENKU & 99 & 465.2245 & 3 & 19.77 & 1392.654 & -1.6 \\
\hline AHFRZWENKU & 99 & 459.8923 & 3 & 20.19 & 1376.659 & -2.9 \\
\hline AHFRZWZNKU & 99 & 508.5823 & 3 & 20.2 & 1522.724 & 1 \\
\hline SHLRQWEYKU & 99 & 415.8863 & 3 & 23.28 & 1244.641 & -3.4 \\
\hline AHLRQWEYKU & 99 & 410.5552 & 3 & 24.23 & 1228.647 & -2.2 \\
\hline SHLZZPEYKU & 99 & 477.228 & 3 & 24.42 & 1428.659 & 1.9 \\
\hline AHLZZPEYKU & 99 & 471.8955 & 3 & 25.82 & 1412.665 & 0.2 \\
\hline AHLZQWENKU & 99 & 430.882 & 3 & 26.7 & 1289.622 & 1.6 \\
\hline SHLRZWEYKU & 99 & 470.2365 & 3 & 26.74 & 1407.69 & -1.7 \\
\hline AHLRZWEYKU & 99 & 464.9048 & 3 & 27.29 & 1391.695 & -1.8 \\
\hline SHFRQWEYKU & 99 & 427.2147 & 3 & 27.73 & 1278.626 & -2.8 \\
\hline SHFZZPEYKU & 99 & 488.5553 & 3 & 27.97 & 1462.644 & 0.2 \\
\hline AHFRQWZYKU & 99 & 470.5727 & 3 & 28.01 & 1408.695 & 0.6 \\
\hline SHLZZWENKU & 99 & 735.3419 & 2 & 28.37 & 1468.666 & 2.5 \\
\hline AZLRQWEYKU & 99 & 448.5694 & 3 & 28.66 & 1342.685 & 1.1 \\
\hline AHLZZWENKU & 99 & 485.2312 & 3 & 28.8 & 1452.671 & 0.7 \\
\hline AHFZZPEYKU & 99 & 483.2245 & 3 & 29.2 & 1446.649 & 1.8 \\
\hline AHFRQWEYKU & 99 & 421.8832 & 3 & 29.45 & 1262.631 & -2.4 \\
\hline SHFZQWENKU & 99 & 447.5413 & 3 & 29.56 & 1339.601 & 0.5 \\
\hline SHFRZWEYKU & 99 & 481.5642 & 3 & 30.25 & 1441.674 & -2.4 \\
\hline
\end{tabular}




\begin{tabular}{|c|c|c|c|c|c|c|}
\hline SHFZZWENKU & 99 & 501.8901 & 3 & 30.84 & 1502.65 & -0.9 \\
\hline AHFRZWEYKU & 99 & 476.2326 & 3 & 31.31 & 1425.679 & -2.4 \\
\hline AHFZZWENKU & 99 & 496.5594 & 3 & 31.83 & 1486.655 & 0.9 \\
\hline SZFRQWEYKU & 99 & 465.2292 & 3 & 32.46 & 1392.664 & 1.3 \\
\hline AZFRQWEYKU & 99 & 459.8966 & 3 & 33.64 & 1376.669 & -0.6 \\
\hline AZFRZWEYKU & 99 & 514.246 & 3 & 35.56 & 1539.718 & -0.9 \\
\hline SZFZZWENKU & 99 & 539.905 & 3 & 36.48 & 1616.688 & 3.3 \\
\hline SHLZZWZYKU & 99 & 555.5932 & 3 & 36.63 & 1663.751 & 4.4 \\
\hline SHLZQWEYKU & 99 & 452.5528 & 3 & 38.13 & 1354.638 & -0.7 \\
\hline AHLZQWEYKU & 99 & 447.2216 & 3 & 39.36 & 1338.643 & 0.3 \\
\hline SHLZZWEYKU & 99 & 506.9029 & 3 & 39.4 & 1517.686 & 0.6 \\
\hline AHLZZWEYKU & 99 & 501.5717 & 3 & 40.32 & 1501.691 & 1.5 \\
\hline SHFZQWEYKU & 99 & 463.8811 & 3 & 41.78 & 1388.622 & -0.2 \\
\hline SHFZZWEYKU & 99 & 518.2308 & 3 & 42.04 & 1551.67 & 0.1 \\
\hline AHFZQWEYKU & 99 & 458.5499 & 3 & 42.97 & 1372.627 & 0.7 \\
\hline AHFZZWEYKU & 99 & 512.8981 & 3 & 43.09 & 1535.675 & -1.8 \\
\hline AHFZQPZNKU & 98 & 461.2243 & 3 & 16.18 & 1380.645 & 4.7 \\
\hline AHFZQPENKU & 98 & 412.5342 & 3 & 16.31 & 1234.58 & 0.8 \\
\hline SZLRQPEYKU & 98 & 424.2242 & 3 & 16.41 & 1269.653 & -1.9 \\
\hline AHLRZWZNKU & 98 & 497.2532 & 3 & 17.44 & 1488.739 & -1 \\
\hline AZLRZPEYKU & 98 & 473.2437 & 3 & 19.77 & 1416.707 & 1.8 \\
\hline AZFRQPEYKU & 98 & 430.2221 & 3 & 20.52 & 1287.643 & 1.5 \\
\hline SZFRQWZNKU & 98 & 497.5778 & 3 & 21.15 & 1489.708 & 2.4 \\
\hline SZLRZWENKU & 98 & 491.9105 & 3 & 22.33 & 1472.708 & 1.3 \\
\hline AZFRZPEYKU & 98 & 484.5714 & 3 & 22.75 & 1450.691 & 0.9 \\
\hline SHLZQPEYKU & 98 & 422.8777 & 3 & 22.78 & 1265.611 & 0.3 \\
\hline AHLZQWZNKU & 98 & 479.5718 & 3 & 24.91 & 1435.687 & 4.9 \\
\hline SHLZQWENKU & 98 & 436.2138 & 3 & 26.07 & 1305.617 & 2 \\
\hline AHFZQPZYKU & 98 & 477.5636 & 3 & 26.38 & 1429.665 & 2.9 \\
\hline AHLRZWZYKU & 98 & 385.4481 & 4 & 26.72 & 1537.76 & 2.4 \\
\hline AHLZZWZNKU & 98 & 533.9205 & 3 & 27.17 & 1598.735 & 2.8 \\
\hline AZLZQPEYKU & 98 & 455.5606 & 3 & 27.3 & 1363.654 & 4.4 \\
\hline SHFZQWZNKU & 98 & 496.2311 & 3 & 27.42 & 1485.666 & 3.7 \\
\hline SZLRQWEYKU & 98 & 453.9011 & 3 & 28.26 & 1358.68 & 1.4 \\
\hline SHFZZWZNKU & 98 & 550.5815 & 3 & 28.7 & 1648.714 & 5.1 \\
\hline AHFZZWZNKU & 98 & 545.2494 & 3 & 29.55 & 1632.72 & 4.2 \\
\hline SZLRZWZYKU & 98 & 556.9402 & 3 & 30.33 & 1667.793 & 3.6 \\
\hline AHFZQWENKU & 98 & 442.2093 & 3 & 30.41 & 1323.606 & -0.4 \\
\hline SZLZZWZNKU & 98 & 577.2672 & 3 & 31.17 & 1728.768 & 6.5 \\
\hline AZFZZPEYKU & 98 & 521.2385 & 3 & 32.92 & 1560.687 & 4.3 \\
\hline SZFRZWEYKU & 98 & 519.5786 & 3 & 35 & 1555.712 & 0.9 \\
\hline SHLZQWZYKU & 98 & 501.2431 & 3 & 35.44 & 1500.702 & 3.8 \\
\hline AHLZQWZYKU & 98 & 495.9115 & 3 & 36.47 & 1484.707 & 3.8 \\
\hline AHLZZWZYKU & 98 & 412.9475 & 4 & 37.51 & 1647.756 & 3.2 \\
\hline SHFZQWZYKU & 98 & 512.571 & 3 & 38.77 & 1534.686 & 3.2 \\
\hline
\end{tabular}




\begin{tabular}{|c|c|c|c|c|c|c|}
\hline SHFZZWZYKU & 98 & 425.4424 & 4 & 39.33 & 1697.735 & 3.2 \\
\hline AZLZZWEYKU & 98 & 539.5858 & 3 & 43.5 & 1615.729 & 3.9 \\
\hline SZLZZWEYKU & 98 & 544.9175 & 3 & 43.7 & 1631.724 & 4 \\
\hline SZFZZWEYKU & 98 & 556.2455 & 3 & 45.93 & 1665.709 & 3.7 \\
\hline SHLRZPEYKU & 97 & 440.5604 & 3 & 15.48 & 1318.663 & -3.1 \\
\hline AZLRQPEYKU & 97 & 418.8932 & 3 & 16.76 & 1253.658 & -0.4 \\
\hline SHLRZWZNKU & 97 & 377.1904 & 4 & 16.88 & 1504.734 & -1.2 \\
\hline SHFRQWZNKU & 97 & 344.9242 & 4 & 17.03 & 1375.67 & -1.6 \\
\hline AZLRQWENKU & 97 & 432.2291 & 3 & 18.43 & 1293.664 & 0.8 \\
\hline SZFZQPENKU & 97 & 455.8793 & 3 & 18.99 & 1364.613 & 2.3 \\
\hline AZFZQPENKU & 97 & 450.5487 & 3 & 19.86 & 1348.618 & 4.5 \\
\hline SZFRQPEYKU & 97 & 435.5536 & 3 & 19.94 & 1303.638 & 1.2 \\
\hline SZFRQWENKU & 97 & 448.8889 & 3 & 21.8 & 1343.644 & 1.1 \\
\hline SZFRZWZNKU & 97 & 551.9274 & 3 & 23.09 & 1652.757 & 2.3 \\
\hline SHLRZWZYKU & 97 & 389.4463 & 4 & 26.21 & 1553.754 & 1 \\
\hline SHLZZWZNKU & 97 & 539.2523 & 3 & 26.34 & 1614.73 & 3.1 \\
\hline AHFRZWZYKU & 97 & 393.9439 & 4 & 29.84 & 1571.744 & 1.6 \\
\hline SZFZQPEYKU & 97 & 472.2201 & 3 & 30.32 & 1413.633 & 3.6 \\
\hline AZLRZWEYKU & 97 & 502.919 & 3 & 31.97 & 1505.733 & 1.4 \\
\hline SZFZZPEYKU & 97 & 526.5698 & 3 & 32.14 & 1576.682 & 3.5 \\
\hline SZFZQWZNKU & 97 & 534.2457 & 3 & 32.54 & 1599.704 & 6.9 \\
\hline SZFZQWZYKU & 97 & 550.5857 & 3 & 42.38 & 1648.725 & 6.5 \\
\hline AHLRQPZYKU & 96 & 429.5692 & 3 & 15.34 & 1285.685 & 0.9 \\
\hline SZLRZPEYKU & 96 & 478.5745 & 3 & 19.04 & 1432.702 & -0.1 \\
\hline AZLRZWZNKU & 96 & 535.2693 & 3 & 21.26 & 1602.777 & 5.5 \\
\hline SHLZQPZYKU & 96 & 471.5681 & 3 & 22.28 & 1411.675 & 5 \\
\hline AHLZQPZYKU & 96 & 466.2354 & 3 & 23.22 & 1395.68 & 2.7 \\
\hline AZFRZWZNKU & 96 & 546.597 & 3 & 23.31 & 1636.762 & 4.5 \\
\hline SHLRQWZYKU & 96 & 348.6837 & 4 & 23.62 & 1390.706 & -0.3 \\
\hline SHLZQWZNKU & 96 & 484.9032 & 3 & 23.95 & 1451.682 & 4.3 \\
\hline SHFZQPZYKU & 96 & 482.8956 & 3 & 25.27 & 1445.66 & 3.6 \\
\hline AHFZZPZYKU & 96 & 531.9138 & 3 & 27.83 & 1592.713 & 3.8 \\
\hline AHFZQWZNKU & 96 & 490.8983 & 3 & 28.18 & 1469.671 & 1.6 \\
\hline SZLZQWZNKU & 96 & 522.9175 & 3 & 29.43 & 1565.72 & 7 \\
\hline AZFZQWENKU & 96 & 480.2245 & 3 & 35.87 & 1437.645 & 4.9 \\
\hline SHFZQPZNKU & 95 & 466.5559 & 3 & 15.47 & 1396.639 & 4.7 \\
\hline AHFRQPZYKU & 95 & 440.8966 & 3 & 16.87 & 1319.669 & -0.6 \\
\hline AZLZZPENKU & 95 & 493.5691 & 3 & 20.38 & 1477.682 & 2.1 \\
\hline AHLZZPZYKU & 95 & 520.5859 & 3 & 24.89 & 1558.729 & 4.4 \\
\hline SHFRQWZYKU & 95 & 357.1795 & 4 & 27.18 & 1424.69 & -0.8 \\
\hline AZLZZPZYKU & 95 & 558.6006 & 3 & 28.33 & 1672.767 & 7.7 \\
\hline SZLZZPEYKU & 95 & 515.2415 & 3 & 28.58 & 1542.698 & 3.4 \\
\hline SZFZQPZYKU & 95 & 520.9103 & 3 & 28.73 & 1559.698 & 7.2 \\
\hline SZFZZPZYKU & 95 & 575.2591 & 3 & 30.66 & 1722.747 & 5.2 \\
\hline SZFRQWZYKU & 95 & 385.6908 & 4 & 31.3 & 1538.729 & 3.6 \\
\hline
\end{tabular}




\begin{tabular}{|c|c|c|c|c|c|c|}
\hline AZFZZPZYKU & 95 & 569.9273 & 3 & 31.6 & 1706.752 & 5.1 \\
\hline AZLZQWENKU & 95 & 468.8958 & 3 & 31.79 & 1403.66 & 3.7 \\
\hline AZLZZWZYKU & 95 & 588.2761 & 3 & 40.95 & 1761.794 & 7.3 \\
\hline AZLZQPZNKU & 94 & 487.9097 & 3 & 16.48 & 1460.698 & 6.2 \\
\hline AZLRQWZNKU & 94 & 360.9406 & 4 & 18.17 & 1439.729 & 3.2 \\
\hline SZLRQWZNKU & 94 & 364.9398 & 4 & 18.29 & 1455.724 & 4.4 \\
\hline SZFZQPZNKU & 94 & 504.5701 & 3 & 18.39 & 1510.678 & 7.3 \\
\hline AZFRZWENKU & 94 & 497.9069 & 3 & 24.6 & 1490.697 & 1 \\
\hline SHFRZWZYKU & 94 & 397.9426 & 4 & 29.14 & 1587.739 & 1.6 \\
\hline SZLZQWZYKU & 94 & 539.2581 & 3 & 39.83 & 1614.74 & 7.6 \\
\hline AHFZQWZYKU & 94 & 380.6817 & 4 & 39.99 & 1518.691 & 4.2 \\
\hline AHFZZWZYKU & 94 & 421.4441 & 4 & 40.29 & 1681.74 & 4.3 \\
\hline SZFZQWEYKU & 94 & 501.8952 & 3 & 45.6 & 1502.66 & 2.5 \\
\hline AHFZZPZNKU & 93 & 515.5734 & 3 & 18.02 & 1543.693 & 3.5 \\
\hline AZLRZWENKU & 93 & 486.579 & 3 & 21.87 & 1456.713 & 1.6 \\
\hline SZFZZPENKU & 93 & 510.2299 & 3 & 22.26 & 1527.662 & 4.1 \\
\hline SZLZQPZYKU & 93 & 509.5818 & 3 & 25.74 & 1525.714 & 6.5 \\
\hline SZLZQPEYKU & 93 & 460.8921 & 3 & 26.64 & 1379.649 & 3.8 \\
\hline SHFZZPZYKU & 93 & 537.2458 & 3 & 26.93 & 1608.708 & 4.6 \\
\hline AZFZQPZYKU & 93 & 515.5787 & 3 & 29.71 & 1543.703 & 7.2 \\
\hline AZLRZWZYKU & 93 & 413.9581 & 4 & 30.65 & 1651.798 & 3.3 \\
\hline AZFZQPEYKU & 93 & 466.8886 & 3 & 31.4 & 1397.638 & 4 \\
\hline AZFRQWZYKU & 93 & 381.6922 & 4 & 32.53 & 1522.734 & 3.9 \\
\hline AZFZQWZNKU & 93 & 528.9144 & 3 & 32.81 & 1583.709 & 7.7 \\
\hline SZFRZWZYKU & 93 & 568.2689 & 3 & 33.38 & 1701.777 & 4.6 \\
\hline SZFZZWZNKU & 93 & 588.5945 & 3 & 33.62 & 1762.753 & 5.2 \\
\hline AZFRZWZYKU & 93 & 422.4547 & 4 & 33.82 & 1685.782 & 4.5 \\
\hline SZLRQPZYKU & 92 & 472.914 & 3 & 16.86 & 1415.718 & 1.9 \\
\hline AZFZQPZNKU & 92 & 499.2379 & 3 & 19.17 & 1494.683 & 6.1 \\
\hline AZFRQWZNKU & 92 & 492.2476 & 3 & 21.69 & 1473.713 & 5.3 \\
\hline SZLRQWZYKU & 92 & 502.5909 & 3 & 28.05 & 1504.744 & 4.6 \\
\hline AZLZQWZNKU & 92 & 517.5864 & 3 & 29.21 & 1549.725 & 8 \\
\hline SZLZZWZYKU & 92 & 593.6075 & 3 & 40.79 & 1777.789 & 6.9 \\
\hline AZFZZWZYKU & 92 & 449.9548 & 4 & 43.36 & 1795.778 & 6.7 \\
\hline AHLZQPZNKU & 91 & 449.8956 & 3 & 14.93 & 1346.66 & 3.6 \\
\hline SHLRQPEYKU & 91 & 386.2108 & 3 & 14.96 & 1155.615 & -3.7 \\
\hline AZLZQPENKU & 91 & 439.219 & 3 & 16.42 & 1314.634 & 1.1 \\
\hline SHLRQWENKU & 91 & 399.5454 & 3 & 16.84 & 1195.621 & -5.6 \\
\hline SZFRQPZYKU & 91 & 484.2436 & 3 & 19.87 & 1449.702 & 4.9 \\
\hline SHLZZPZYKU & 91 & 525.9167 & 3 & 23.57 & 1574.724 & 2.8 \\
\hline AZLRQPZYKU & 90 & 467.5824 & 3 & 17.39 & 1399.723 & 1.9 \\
\hline SZLRQWENKU & 90 & 437.5607 & 3 & 18.7 & 1309.659 & 0.8 \\
\hline AZFZQWZYKU & 90 & 545.2544 & 3 & 43 & 1632.73 & 7.3 \\
\hline AHLRQPEYKU & 89 & 380.8794 & 3 & 15.25 & 1139.62 & -3 \\
\hline AZLZQPZYKU & 89 & 504.2508 & 3 & 26.68 & 1509.719 & 7.9 \\
\hline
\end{tabular}




\begin{tabular}{|c|c|c|c|c|c|c|}
\hline AZFZZWZNKU & 89 & 583.2639 & 3 & 34.02 & 1746.758 & 6.8 \\
\hline AZFRZPENKU & 88 & 468.231 & 3 & 14.85 & 1401.671 & 0.5 \\
\hline SHLRQPZYKU & 87 & 434.901 & 3 & 14.94 & 1301.679 & 1.3 \\
\hline SZLRZWZNKU & 87 & 405.7011 & 4 & 21.17 & 1618.772 & 1.9 \\
\hline AZLZZWZNKU & 87 & 571.9355 & 3 & 31.19 & 1712.773 & 6.5 \\
\hline AZFZZWENKU & 87 & 534.5741 & 3 & 36.59 & 1600.693 & 4.6 \\
\hline AZFZZWEYKU & 87 & 550.9141 & 3 & 46.35 & 1649.714 & 4.1 \\
\hline AHLRZPZYKU & 86 & 363.1913 & 4 & 16.46 & 1448.733 & 2.3 \\
\hline AHFRZPEYKU & 86 & 335.1693 & 4 & 19.11 & 1336.653 & -3.5 \\
\hline AHLRQWZYKU & 86 & 344.6856 & 4 & 24.27 & 1374.711 & 1.8 \\
\hline SZFZZWZYKU & 86 & 453.9525 & 4 & 42.87 & 1811.773 & 4.4 \\
\hline AZFZQWEYKU & 86 & 496.5642 & 3 & 46.25 & 1486.665 & 3.8 \\
\hline AZLZQWZYKU & 85 & 533.9264 & 3 & 40.01 & 1598.745 & 7.7 \\
\hline SZLZQWEYKU & 85 & 490.5679 & 3 & 42.53 & 1468.676 & 4.3 \\
\hline SZFRZPZNKU & 84 & 391.9414 & 4 & 14.84 & 1563.73 & 4.2 \\
\hline SHFZQPEYKU & 84 & 650.8057 & 2 & 26.75 & 1299.595 & 1.3 \\
\hline AHFZQPEYKU & 84 & 642.8075 & 2 & 27.99 & 1283.6 & 0.1 \\
\hline SZLZZWENKU & 84 & 528.5765 & 3 & 34.53 & 1582.704 & 2.5 \\
\hline AZLZZPEYKU & 83 & 509.9105 & 3 & 29.26 & 1526.703 & 4.6 \\
\hline AZLZQWEYKU & 83 & 485.2364 & 3 & 42.67 & 1452.681 & 4.7 \\
\hline AZFRQWENKU & 82 & 443.5575 & 3 & 22.43 & 1327.649 & 1.5 \\
\hline AZLZZWENKU & 82 & 523.2454 & 3 & 33.87 & 1566.709 & 3.6 \\
\hline AZLRQWZYKU & 81 & 373.1962 & 4 & 28.33 & 1488.749 & 4.3 \\
\hline AHLRZWENKU & 79 & 448.5627 & 3 & 16.44 & 1342.675 & -6.2 \\
\hline AZFZZPENKU & 79 & 504.8978 & 3 & 22.77 & 1511.667 & 3.2 \\
\hline SZLRZPZYKU & 78 & 527.2649 & 3 & 19.43 & 1578.766 & 4.3 \\
\hline AHLRQWENKU & 77 & 394.2137 & 3 & 16.84 & 1179.626 & -5.8 \\
\hline AZFRZPZYKU & 77 & 533.2614 & 3 & 22.66 & 1596.756 & 4.1 \\
\hline SHLRZPZYKU & 76 & 367.1899 & 4 & 16.07 & 1464.728 & 1.7 \\
\hline AHLZQPEYKU & 76 & 625.8153 & 2 & 23.89 & 1249.616 & 0.1 \\
\hline SHFRZPZYKU & 75 & 375.6859 & 4 & 18.41 & 1498.712 & 1.4 \\
\hline SZFRZWENKU & 74 & 503.238 & 3 & 24.15 & 1506.692 & 0 \\
\hline AZFRQPZYKU & 70 & 359.4358 & 4 & 20.54 & 1433.707 & 4.9 \\
\hline SHFRQPZYKU & 67 & 334.9227 & 4 & 16.45 & 1335.664 & -1.7 \\
\hline SZLRZWEYKU & 67 & 381.4389 & 4 & 31.65 & 1521.728 & -1.1 \\
\hline SZLZQPZNKU & 66 & 493.2416 & 3 & 16.05 & 1476.693 & 6.6 \\
\hline AHFRZPZYKU & 64 & 495.2473 & 3 & 19.42 & 1482.717 & 1.8 \\
\hline AZLRZPZYKU & 64 & 391.7016 & 4 & 20.15 & 1562.771 & 3.8 \\
\hline SHFRZPEYKU & 63 & 451.8883 & 3 & 18.05 & 1352.648 & -3.3 \\
\hline SZFRZPEYKU & 56 & 367.6793 & 4 & 22.23 & 1466.686 & 1.3 \\
\hline
\end{tabular}


Synthesis and characterization of a $10^{8}$-member nucleobase peptide library

Library design: $(\mathrm{X})_{9} \mathrm{~K}-\mathrm{CONH}_{2}$ with $\mathrm{X}=18$ canonical amino acids (excluded Cys and lle) \& DabA, DabC, DabG, DabT, AlaA, AlaC, AlaG, AlaT, hAlaA, hAlaC, hAlaG, hAlaT.

\section{Library synthesis: SPPS:}

$2.2 \mathrm{~g}$ of $30 \mu \mathrm{m}$ TentaGel resin $\left(0.26 \mathrm{mmol} / \mathrm{g}, 0.74 \mathrm{mmol}, 1.0 \times 10^{8}\right.$ beads) was transferred to a $25 \mathrm{~mL}$ peptide synthesis vessel, swollen in DMF, and then washed with DMF (3x). Fmoc-Rink amide linker (5 eq) was dissolved in HATU solution (0.38 M in DMF) activated with DIEA (1.86 mL, $10.7 \mathrm{mmol}$ ) immediately prior to coupling, and added to resin bed. Coupling was performed for $20 \mathrm{~min}$; after this time, resin was washed with DMF (100 mL). Fmoc removal was carried out by treatment of resin with $20 \%$ piperidine in DMF ( $1 \times 30 \mathrm{~mL}$ flow wash; $1 \times 15 \mathrm{~mL}$, 5 min batch treatments). Resin was then washed with DMF (150 $\mathrm{mL}$ ). Coupling of Fmoc-Lys(Boc)-OH, subsequent Fmoc removal, and DMF washes were performed in the same manner.

At this stage, resin was suspended in DMF $(10 \mathrm{~mL})$, and divided in two parts ( $20 \%$ and $80 \%$, respectively). The $20 \%$ part was divided evenly among $12 \times 10 \mathrm{~mL}$ fritted plastic syringes and the $80 \%$ part was divided evenly among $18 \times 10 \mathrm{~mL}$ fritted plastic syringes using a $5 \mathrm{~mL}$ Eppendorf pipette. Couplings were performed according to the procedure described in "Peptide synthesis procedures". After coupling for 20 min, resins were washed with DMF ( $10 \mathrm{~mL}$ ea.), poured back into a $25 \mathrm{~mL}$ synthesis vessel, and washed with DMF $(100 \mathrm{~mL}$ ). Fmoc removal was carried out by treatment of resin with $20 \%$ piperidine in DMF ( $1 \mathrm{x}$ $30 \mathrm{~mL}$ flow wash; $1 \times 15 \mathrm{~mL}, 5 \mathrm{~min}$ batch treatments). Resin was then washed with DMF (150 mL). Nine cycles of split-and-pool synthesis were performed using this procedure.

Cleavage and solid phase extraction were performed according to procedures described in section "Peptide synthesis procedures".

$450 \mathrm{mg}$ lyophilized powder ( 60\% crude yield) were obtained.

The library was dissolved in $1 \mathrm{xPBS} \mathrm{pH}=7.2+5 \% \mathrm{DMF}$, aliquoted and stored at $-80^{\circ} \mathrm{C}$.

Library QC: A $1.0 \mathrm{mg}$ aliquot of library resin (from Section 6.1) was suspended in $1.0 \mathrm{~mL}$ of Milli-Q water and sonicated to achieve a homogenous suspension (theory: $3 \times 10^{4}$ beads $/ \mathrm{mL}$ ). A $20 \mu \mathrm{L}$ aliquot (theory: 650 beads; 4 pmol/peptide) was transferred to a plastic tube, spun down, and supernatant removed. Beads were then subjected to nucleobase peptide cleavage conditions. TFA was then evaporated under a stream of nitrogen, and cleaved peptide was resuspended in Milli-Q water (0.1\% TFA). Sample was purified over a C18 ZipTip ${ }^{\circledR}(0.6 \mu \mathrm{L}$, MilliporeSigma, P/N ZTC18S096), eluted in 30/70 water/acetonitrile ( $0.1 \%$ TFA), and lyophilized. Powder was resuspended in $20 \mu \mathrm{L}$ of Milli-Q water ( $0.1 \% \mathrm{FA})$, and $0.5 \mu \mathrm{L}$ ( 100 $\mathrm{fmol} /$ peptide) was submitted for nLC-MS/MS analysis. 
Library QC sample 1

$U=C$-terminal amide, $B=D a b A, C=D a b C, I=D a b G, J=D a b T, O=A l a A, U=A l a C, X=A l a G, Z=A l a T$, $b=h A l a A, c=h A l a C, i=h A l a G, j=h A l a T$

\begin{tabular}{|c|c|c|c|c|c|c|}
\hline Peptide & $A L C(\%)$ & $m / z$ & $z$ & $R T$ & Mass & ppm \\
\hline HJbADFFEDKU & 99 & 497.8781 & 3 & 43.69 & 1490.635 & -15 \\
\hline CVQMFCAFDKU & 99 & 669.7984 & 2 & 46.54 & 1337.596 & -10.4 \\
\hline LOASAWAJFKU & 98 & 454.8922 & 3 & 32.48 & 1361.67 & -10.9 \\
\hline VDCTYCFNbKU & 98 & 486.546 & 3 & 33.38 & 1456.633 & -11.2 \\
\hline DGSVLYAFCKU & 98 & 551.2668 & 2 & 49.42 & 1100.533 & -12.1 \\
\hline GMFGGEYCSKU & 98 & 409.1783 & 3 & 51.16 & 1224.53 & -13.5 \\
\hline GVVEPMFbVKU & 98 & 408.2183 & 3 & 68.03 & 1221.64 & -5.5 \\
\hline TCSSNAEBiKU & 97 & 449.8585 & 3 & 24.64 & 1346.559 & -4.2 \\
\hline DQGjDLXVKKU & 97 & 444.2195 & 3 & 25.73 & 1329.651 & -10.6 \\
\hline DSTCAYGAVKU & 97 & 338.4905 & 3 & 27.2 & 1012.465 & -15 \\
\hline DMSTCAKFIKU & 97 & 440.8616 & 3 & 27.32 & 1319.585 & -17 \\
\hline QAPELLjEGKU & 97 & 398.2072 & 3 & 29.94 & 1191.622 & -18.6 \\
\hline DDHDYGVOQKU & 97 & 427.1839 & 3 & 33.26 & 1278.549 & -14.8 \\
\hline YKYEGTjPCKU & 97 & 482.2274 & 3 & 33.44 & 1443.682 & -14.8 \\
\hline ITEGAKWTRKU & 97 & 456.2318 & 3 & 35.03 & 1365.7 & -19.8 \\
\hline CFYNDJTXGKU & 97 & 527.5525 & 3 & 40.09 & 1579.657 & -13.7 \\
\hline DMTCVVKFTKU & 97 & 390.8661 & 3 & 41.13 & 1169.594 & -14.8 \\
\hline TjHjJGEAYKU & 97 & 496.8826 & 3 & 42.2 & 1487.644 & -12 \\
\hline PMAEDEAYTKU & 97 & 577.2566 & 2 & 47.46 & 1152.512 & -11.8 \\
\hline YVNMLYTCAKU & 97 & 402.1944 & 3 & 47.54 & 1203.578 & -14 \\
\hline PAGVPMWWDKU & 97 & 395.8594 & 3 & 49.31 & 1184.58 & -20.1 \\
\hline AGQOGPBLCKU & 97 & 671.8226 & 2 & 52.28 & 1341.657 & -19.7 \\
\hline LEFALLARHKU & 97 & 399.5758 & 3 & 53.32 & 1195.719 & -11.2 \\
\hline MPVADGVJRKU & 96 & 413.2117 & 3 & 23.55 & 1236.635 & -17.7 \\
\hline TDZADRNAAKU & 96 & 385.8484 & 3 & 27.4 & 1154.543 & -16.6 \\
\hline jQcDYHWATKU & 96 & 484.2166 & 3 & 36.32 & 1449.648 & -13.5 \\
\hline IYGMDELQKKU & 96 & 467.8851 & 3 & 40.52 & 1400.661 & -19.8 \\
\hline LKGYSNADjKU & 96 & 401.8675 & 3 & 40.55 & 1202.601 & -17.2 \\
\hline TCDTWYTKXKU & 96 & 455.5405 & 3 & 42.49 & 1363.609 & -7 \\
\hline NMFLRCRYVKU & 96 & 443.5684 & 3 & 46.21 & 1327.7 & -12.9 \\
\hline PMAPMCPYTKU & 96 & 569.259 & 2 & 47.35 & 1136.518 & -12.8 \\
\hline EGSYTciTKKU & 96 & 447.5533 & 3 & 49.56 & 1339.636 & 2 \\
\hline JSNjDALPDKU & 96 & 667.2993 & 2 & 53.21 & 1332.598 & -10.8 \\
\hline FTFQSMQMMKU & 96 & 639.2849 & 2 & 69.43 & 1276.577 & -16.8 \\
\hline VjFGEWEIVKU & 96 & 498.2304 & 3 & 71.77 & 1491.697 & -18.3 \\
\hline EMADYQSVIKU & 96 & 652.2917 & 2 & 80.55 & 1302.578 & -7 \\
\hline PRJZRQGEJKU & 95 & 532.9215 & 3 & 23.88 & 1595.758 & -9.5 \\
\hline
\end{tabular}




\begin{tabular}{|c|c|c|c|c|c|c|}
\hline VECSLHWVYKU & 95 & 470.9064 & 3 & 31.09 & 1409.716 & -13 \\
\hline EMZASMDMDKU & 95 & 417.8246 & 3 & 31.12 & 1250.473 & -16.6 \\
\hline TNFCTJANjKU & 95 & 458.1997 & 3 & 32.19 & 1371.592 & -10.3 \\
\hline LOASAQEJFKU & 95 & 454.8914 & 3 & 32.75 & 1361.654 & -1.5 \\
\hline PYRFTCPPGKU & 95 & 388.8654 & 3 & 32.84 & 1163.591 & -14.2 \\
\hline DPMCCVSEVKU & 95 & 419.8554 & 3 & 33.07 & 1256.559 & -11.9 \\
\hline DDHYDGVZHKU & 95 & 427.1839 & 3 & 33.1 & 1278.538 & -6.2 \\
\hline QbEVJSEVEKU & 95 & 477.5577 & 3 & 33.49 & 1429.661 & -6.7 \\
\hline MNCFVALBRKU & 95 & 452.5613 & 3 & 33.57 & 1354.68 & -13.5 \\
\hline VGANDSJAMKU & 95 & 550.7554 & 2 & 41.93 & 1099.505 & -8.1 \\
\hline TjHjYCWAYKU & 95 & 496.8824 & 3 & 42.27 & 1487.635 & -6.1 \\
\hline FQLTBVAPVKU & 95 & 319.9297 & 4 & 44.87 & 1275.714 & -19.1 \\
\hline GMVQWQWKMKU & 95 & 440.894 & 3 & 50.43 & 1319.663 & -2.3 \\
\hline PLYDGETGEKU & 95 & 554.2612 & 2 & 55.09 & 1106.524 & -14.9 \\
\hline UDPYQNKQYKU & 95 & 681.8253 & 2 & 63.95 & 1361.648 & -8.5 \\
\hline NCSMAADCJKU & 95 & 723.8056 & 2 & 64.19 & 1445.609 & -8.8 \\
\hline TjFjJADAYKU & 95 & 749.8266 & 2 & 65.95 & 1497.653 & -9.8 \\
\hline ACGVQWWXLKU & 95 & 437.2103 & 3 & 66.97 & 1308.63 & -15.7 \\
\hline FDFLKZMSSKU & 95 & 432.8791 & 3 & 70.11 & 1295.633 & -13.8 \\
\hline TPSLVADTFKU & 95 & 539.2932 & 2 & 73.29 & 1076.587 & -13.7 \\
\hline FFAGYLVGXKU & 95 & 610.8096 & 2 & 73.7 & 1219.625 & -16.7 \\
\hline FFTMLTKIHKU & 95 & 481.5804 & 3 & 77.39 & 1441.739 & -13.7 \\
\hline SGMDTTUbRKU & 94 & 431.5294 & 3 & 25.28 & 1291.591 & -18.8 \\
\hline HEMJSMDMDKU & 94 & 463.5095 & 3 & 27.34 & 1387.528 & -15.2 \\
\hline EMDKSQPGEKU & 94 & 383.1786 & 3 & 30.14 & 1146.534 & -17.4 \\
\hline VECSLKNUYKU & 94 & 470.9061 & 3 & 31.04 & 1409.712 & -10.8 \\
\hline KVFQIWVGTKU & 94 & 461.5847 & 3 & 31.35 & 1381.736 & -2.6 \\
\hline LOVLVDQDQKU & 94 & 420.898 & 3 & 32.46 & 1259.673 & -1 \\
\hline GYFOWRTGDKU & 94 & 444.8757 & 3 & 34.79 & 1331.627 & -16.5 \\
\hline DYDTDQFYNKU & 94 & 436.5208 & 3 & 40.49 & 1306.547 & -4.7 \\
\hline ODTADYTKXKU & 94 & 455.5395 & 3 & 42.77 & 1363.613 & -11.8 \\
\hline CVGQEGYJMKU & 94 & 476.5453 & 3 & 48.75 & 1426.632 & -12.5 \\
\hline YZFCDFASEKU & 94 & 652.2653 & 2 & 49.32 & 1302.534 & -13.7 \\
\hline TPSLVJGTFKU & 94 & 405.5487 & 3 & 56.89 & 1213.641 & -13.9 \\
\hline DFILENDSEKU & 94 & 462.8654 & 3 & 58.95 & 1385.595 & -14.8 \\
\hline WLKLIMYIAKU & 94 & 545.2761 & 3 & 61.12 & 1632.819 & -7.8 \\
\hline GbDDYVLLQKU & 94 & 423.216 & 3 & 65.06 & 1266.642 & -12.8 \\
\hline SSGVQEGWLKU & 94 & 545.2793 & 2 & 67.88 & 1088.562 & -16.1 \\
\hline bCFLSVDYNKU & 94 & 435.8759 & 3 & 73.92 & 1304.604 & 1.5 \\
\hline ZQTCMEQSDKU & 93 & 632.2498 & 2 & 24.08 & 1262.502 & -13.4 \\
\hline WbTFAVGGKKU & 93 & 404.2153 & 3 & 25.73 & 1209.648 & -19.3 \\
\hline XiHWAARAGKU & 93 & 338.1688 & 4 & 26.07 & 1348.652 & -4 \\
\hline LEGEQRTjKKU & 93 & 432.8966 & 3 & 26.67 & 1295.692 & -18.3 \\
\hline LNSPZDCVEKU & 93 & 599.7719 & 2 & 27.85 & 1197.545 & -13.2 \\
\hline KEYDYNVXEKU & 93 & 352.4145 & 4 & 28.37 & 1405.638 & -6.1 \\
\hline
\end{tabular}




\begin{tabular}{|c|c|c|c|c|c|c|}
\hline ADQSMSTVFKU & 93 & 371.5124 & 3 & 29.27 & 1111.533 & -16.1 \\
\hline DVGWJEIGVKU & 93 & 482.5495 & 3 & 33.72 & 1444.654 & -19.1 \\
\hline jTUGKbCRMKU & 93 & 477.2228 & 3 & 35.64 & 1428.665 & -13 \\
\hline ccFGVYMADKU & 93 & 439.8673 & 3 & 36.1 & 1316.602 & -16.6 \\
\hline TCCVLETjiKU & 93 & 496.2341 & 3 & 38.51 & 1485.682 & -1.3 \\
\hline POMPNANVjKU & 93 & 428.2056 & 3 & 40.37 & 1281.612 & -13.1 \\
\hline CTNDHFFTYKU & 93 & 425.519 & 3 & 41.42 & 1273.555 & -15.5 \\
\hline DGQXKTADOKU & 93 & 429.1969 & 3 & 43.17 & 1284.582 & -10 \\
\hline FADESVBLVKU & 93 & 427.8877 & 3 & 62.19 & 1280.657 & -12 \\
\hline CHEFPAPMRKU & 93 & 435.8859 & 3 & 63.13 & 1304.653 & -12.9 \\
\hline ijDATMPEGKU & 93 & 645.7826 & 2 & 64.61 & 1289.555 & -3.1 \\
\hline EGOLNAYADKU & 93 & 395.1899 & 3 & 65.26 & 1182.553 & -4.2 \\
\hline FDFLKYCFSKU & 93 & 432.8796 & 3 & 68.89 & 1295.637 & -15.7 \\
\hline CVEAVKAAjKU & 93 & 425.5674 & 3 & 73.6 & 1273.681 & -0.9 \\
\hline CPELWLDDAKU & 93 & 594.7892 & 2 & 82.07 & 1187.565 & -0.4 \\
\hline NLGEYXOGFKU & 93 & 675.8098 & 2 & 83.05 & 1349.613 & -5.5 \\
\hline EMDAYQSFIKU & 93 & 676.2924 & 2 & 91.72 & 1350.578 & -5.9 \\
\hline VPGVLVCQQKU & 92 & 305.1761 & 4 & 25.04 & 1216.699 & -19.8 \\
\hline VEIRVRJADKU & 92 & 491.2498 & 3 & 28.32 & 1470.752 & -16.3 \\
\hline bVVLVDGWQKU & 92 & 420.8978 & 3 & 32.35 & 1259.684 & -9.9 \\
\hline MGMjEOCLKKU & 92 & 451.2036 & 3 & 34.35 & 1350.608 & -13.9 \\
\hline XAPOVYMADKU & 92 & 439.8667 & 3 & 36.13 & 1316.595 & -12.4 \\
\hline XIGMEDLQKKU & 92 & 467.8853 & 3 & 40.6 & 1400.648 & -10.4 \\
\hline CVGAMSYbDKU & 92 & 446.867 & 3 & 46.57 & 1337.596 & -12.1 \\
\hline DFQLEQCTFKU & 92 & 419.8625 & 3 & 61.34 & 1256.586 & -16 \\
\hline UPHPYSKLEKU & 92 & 426.5623 & 3 & 63.33 & 1276.668 & -1.8 \\
\hline MMFLKYFCSKU & 92 & 432.8787 & 3 & 70.27 & 1295.623 & -6.6 \\
\hline VPLLVCADBKU & 92 & 661.8654 & 2 & 92.72 & 1321.728 & -8.5 \\
\hline TYQTFQPGVKU & 91 & 389.8689 & 3 & 26.82 & 1166.608 & -20.2 \\
\hline KYCTBGQJYKU & 91 & 510.8956 & 3 & 26.96 & 1529.684 & -12.7 \\
\hline KTHQDMiMWKU & 91 & 479.8886 & 3 & 29.85 & 1436.656 & -8.4 \\
\hline INEMIVSPSKU & 91 & 491.552 & 3 & 32.28 & 1471.647 & -8.7 \\
\hline VDTCYCFHZKU & 91 & 486.546 & 3 & 33.47 & 1456.626 & -6.8 \\
\hline AJCDYHAWTKU & 91 & 484.2182 & 3 & 36.39 & 1449.646 & -9.1 \\
\hline LYDISVMGRKU & 91 & 340.4185 & 4 & 38.59 & 1357.667 & -16 \\
\hline HHQDMjFGOKU & 91 & 471.2018 & 3 & 41.86 & 1410.608 & -17.4 \\
\hline PLYDZQTGEKU & 91 & 415.5276 & 3 & 42.34 & 1243.583 & -18 \\
\hline BNWQCYRGNKU & 91 & 511.9114 & 3 & 43.18 & 1532.715 & -1.9 \\
\hline MFAQPGMQFKU & 91 & 592.2835 & 2 & 49.21 & 1182.568 & -13 \\
\hline MGMјEXQKKKU & 91 & 469.8891 & 3 & 50.01 & 1406.663 & -12.3 \\
\hline FGSLbGSPEKU & 91 & 569.7822 & 2 & 56.53 & 1137.564 & -11.9 \\
\hline VcEQCTEVYKU & 91 & 720.3445 & 2 & 60.76 & 1438.687 & -8.8 \\
\hline LZSMLTZjLKU & 91 & 468.5686 & 3 & 62.65 & 1402.7 & -11.2 \\
\hline SDLAScZDSKU & 91 & 605.7681 & 2 & 74.97 & 1209.534 & -10.4 \\
\hline MEDAYQSWIKU & 91 & 695.7971 & 2 & 91.38 & 1389.589 & -6.6 \\
\hline
\end{tabular}




\begin{tabular}{|c|c|c|c|c|c|c|}
\hline ZPFLQNQAKKU & 90 & 423.2333 & 3 & 24.6 & 1266.683 & -4.1 \\
\hline PSGFCGYAGKU & 90 & 493.2245 & 2 & 25.33 & 984.4487 & -14.5 \\
\hline VYGNHLQHPKU & 90 & 397.8793 & 3 & 27.03 & 1190.631 & -12.5 \\
\hline PMIRVRJADKU & 90 & 491.2498 & 3 & 28.33 & 1470.734 & -4.2 \\
\hline YASPETAHFKU & 90 & 383.8586 & 3 & 28.43 & 1148.562 & -6.5 \\
\hline YTNCOQQWEKU & 90 & 468.198 & 3 & 33.93 & 1401.6 & -19.5 \\
\hline LLEDMLANLKU & 90 & 290.4155 & 4 & 34.28 & 1157.648 & -12.9 \\
\hline QBPXGYYDMKU & 90 & 499.2083 & 3 & 38.9 & 1494.626 & -15.5 \\
\hline GGEDGRLLVKU & 90 & 348.2002 & 3 & 39.93 & 1041.593 & -13.6 \\
\hline XiGMNNLQKKU & 90 & 462.5545 & 3 & 40.4 & 1384.665 & -16.8 \\
\hline MKVLTCNVMKU & 90 & 389.2113 & 3 & 41.49 & 1164.618 & -5.3 \\
\hline DYEDJGVNFKU & 90 & 451.199 & 3 & 42.36 & 1350.58 & -3.3 \\
\hline BDIGKAFDAKU & 90 & 472.8883 & 3 & 49.75 & 1415.649 & -3.9 \\
\hline KGMLFjjVDKU & 90 & 452.2285 & 3 & 50.38 & 1353.68 & -12.3 \\
\hline KTSLFjQUDKU & 90 & 452.2279 & 3 & 50.52 & 1353.676 & -10.4 \\
\hline KFXCLRIEFKU & 90 & 527.5922 & 3 & 55.51 & 1579.768 & -8.5 \\
\hline ZWAOVISYFKU & 90 & 530.5729 & 3 & 58.13 & 1588.718 & -13 \\
\hline MMQLEQTCFKU & 90 & 419.8619 & 3 & 61.24 & 1256.572 & -6.1 \\
\hline FBEFJADRFKU & 90 & 400.6863 & 4 & 63.43 & 1598.739 & -14.2 \\
\hline VFQZMDSZLKU & 90 & 678.8128 & 2 & 69.63 & 1355.629 & -13.3 \\
\hline YNETTYDTEKU & 90 & 631.7781 & 2 & 71.2 & 1261.546 & -3.5 \\
\hline NLEGYXFGOKU & 90 & 675.8093 & 2 & 83.26 & 1349.613 & -6.3 \\
\hline KiSLOHAIYKU & 90 & 506.5835 & 3 & 88.87 & 1516.742 & -8.7 \\
\hline PYFNRPcSSKU & 89 & 322.9151 & 4 & 24.32 & 1287.644 & -10 \\
\hline UBGVENEFHKU & 89 & 471.8797 & 3 & 26.8 & 1412.639 & -15.2 \\
\hline YVKGJCRENKU & 89 & 378.1918 & 4 & 27.65 & 1508.751 & -8.1 \\
\hline MCSTLENMCKU & 89 & 436.1882 & 3 & 28.12 & 1305.558 & -11.8 \\
\hline WDEDHAFVGKU & 89 & 401.5187 & 3 & 28.19 & 1201.552 & -14.4 \\
\hline KGRTUPMTNKU & 89 & 404.5456 & 3 & 31.01 & 1210.635 & -16.7 \\
\hline PKJETAEPGKU & 89 & 388.8654 & 3 & 32.84 & 1163.591 & -14 \\
\hline GVTPGAZFEKU & 89 & 367.1838 & 3 & 36.14 & 1098.546 & -14.8 \\
\hline WFKNMGNYDKU & 89 & 434.5359 & 3 & 36.96 & 1300.602 & -12.7 \\
\hline PTSCTSIFWKU & 89 & 449.532 & 3 & 40.01 & 1345.598 & -17.4 \\
\hline CVQMCYAFDKU & 89 & 452.1984 & 3 & 46.64 & 1353.591 & -13.1 \\
\hline WVBYDNFMGKU & 89 & 478.5509 & 3 & 47.43 & 1432.64 & -6.4 \\
\hline ETNMZIGFJKU & 89 & 526.5522 & 3 & 48.54 & 1576.654 & -12.1 \\
\hline HQDUGCFNFKU & 89 & 637.7726 & 2 & 48.82 & 1273.541 & -8.2 \\
\hline MYGFMTSTCKU & 89 & 584.2464 & 2 & 50.43 & 1166.492 & -12 \\
\hline DVTTGbWFGKU & 89 & 409.867 & 3 & 50.91 & 1226.59 & -8.9 \\
\hline HDBLEQCTFKU & 89 & 465.5481 & 3 & 51.11 & 1393.625 & -1.8 \\
\hline MVAFUVHTLKU & 89 & 408.8902 & 3 & 52.5 & 1223.66 & -8.9 \\
\hline EGQFDAESKKU & 89 & 379.8546 & 3 & 54 & 1136.546 & -3.6 \\
\hline FTFQMCQDFKU & 89 & 647.2827 & 2 & 54.9 & 1292.568 & -13.3 \\
\hline FSjDATDEWKU & 89 & 653.778 & 2 & 56.12 & 1305.56 & -14 \\
\hline WGDSDYTAQKU & 89 & 585.259 & 2 & 58.28 & 1168.515 & -9 . \\
\hline
\end{tabular}




\begin{tabular}{|c|c|c|c|c|c|c|}
\hline DXbLENDSEKU & 89 & 462.865 & 3 & 59.13 & 1385.577 & -3.1 \\
\hline VFQZMDSALKU & 89 & 616.8004 & 2 & 64.63 & 1231.602 & -12.9 \\
\hline OGMYFjLOcKU & 89 & 784.8489 & 2 & 66.93 & 1567.705 & -14 \\
\hline PUETTYDTEKU & 89 & 631.778 & 2 & 71.28 & 1261.557 & -12.6 \\
\hline WMAYDEEMMKU & 89 & 666.7696 & 2 & 83.19 & 1331.535 & -7.7 \\
\hline KDHMPDWHDKU & 88 & 436.5341 & 3 & 23.22 & 1306.588 & -5.4 \\
\hline GVSHMPDIOKU & 88 & 436.5341 & 3 & 23.27 & 1306.585 & -3.8 \\
\hline EDVADRAOFKU & 88 & 418.5429 & 3 & 23.65 & 1252.606 & 0.7 \\
\hline YNSLMNIMEKU & 88 & 454.8643 & 3 & 26.89 & 1361.597 & -19.4 \\
\hline GXSLMNIMEKU & 88 & 454.864 & 3 & 26.98 & 1361.584 & -9.8 \\
\hline LGYAETTSMKU & 88 & 367.1816 & 3 & 28.75 & 1098.538 & -13.5 \\
\hline STADAKWTRKU & 88 & 388.209 & 3 & 30.09 & 1161.626 & -17.6 \\
\hline PNLRCQMGTKU & 88 & 382.8637 & 3 & 31.63 & 1145.58 & -9.1 \\
\hline KARMMPANLKU & 88 & 386.8847 & 3 & 33.91 & 1157.653 & -17.5 \\
\hline bGSTLDWJEKU & 88 & 473.5492 & 3 & 36.18 & 1417.64 & -10 \\
\hline GVSFMPDIOKU & 88 & 439.8704 & 3 & 37.84 & 1316.595 & -4.3 \\
\hline KDEQXXYDMKU & 88 & 499.2081 & 3 & 39.16 & 1494.617 & -9.7 \\
\hline OSXMNNLQKKU & 88 & 462.5541 & 3 & 40.67 & 1384.664 & -17.3 \\
\hline AEEAPLAAAKU & 88 & 485.2659 & 2 & 43.13 & 968.5291 & -12.3 \\
\hline ALDMDYjMEKU & 88 & 441.856 & 3 & 44.62 & 1322.561 & -10.8 \\
\hline IGEjAVNJSKU & 88 & 490.5491 & 3 & 44.65 & 1468.648 & -15 \\
\hline PLYDOYGGEKU & 88 & 415.5272 & 3 & 44.87 & 1243.573 & -10.9 \\
\hline iPSZFDGVGKU & 88 & 412.1923 & 3 & 46.33 & 1233.565 & -7.7 \\
\hline jMMSGDZSLKU & 88 & 424.5179 & 3 & 50.07 & 1270.541 & -6.8 \\
\hline UNMSMYDDEKU & 88 & 656.2488 & 2 & 54.17 & 1310.502 & -14.4 \\
\hline ZWAFRBCYFKU & 88 & 530.5732 & 3 & 57.89 & 1588.72 & -13.9 \\
\hline VYADTYVNAKU & 88 & 571.7902 & 2 & 58.46 & 1141.577 & -9.5 \\
\hline DFIQNNMAEKU & 88 & 693.7954 & 2 & 59.15 & 1385.589 & -8.9 \\
\hline EZDMSEMENKU & 88 & 653.7473 & 2 & 59.99 & 1305.497 & -12.7 \\
\hline GLDNLVbOZKU & 88 & 458.8907 & 3 & 62.15 & 1373.677 & -19.4 \\
\hline EGFESVBLVKU & 88 & 427.8863 & 3 & 62.31 & 1280.657 & -15.2 \\
\hline VTLYZTAXEKU & 88 & 669.825 & 2 & 73.88 & 1337.648 & -9.1 \\
\hline DSVFLZMYTKU & 88 & 649.3071 & 2 & 77.47 & 1296.617 & -13.5 \\
\hline FMAWLGYDQKU & 88 & 629.2974 & 2 & 90.75 & 1256.601 & -16.6 \\
\hline GbTVQSMMTKU & 87 & 400.5235 & 3 & 25.27 & 1198.565 & -13.9 \\
\hline TDCTBKELYKU & 87 & 458.8841 & 3 & 27.94 & 1373.645 & -10.5 \\
\hline TGGANGWYiKU & 87 & 396.1849 & 3 & 27.96 & 1185.544 & -8.9 \\
\hline QRPANLLACKU & 87 & 420.9104 & 3 & 27.98 & 1259.716 & -5.4 \\
\hline HPCFQDPSWKU & 87 & 348.6682 & 4 & 30.88 & 1390.648 & -3.4 \\
\hline KARTEEANLKU & 87 & 386.8847 & 3 & 34.13 & 1157.652 & -16.7 \\
\hline GDQXKTGEOKU & 87 & 429.198 & 3 & 39.87 & 1284.582 & -7.4 \\
\hline MFEDYCRNFKU & 87 & 676.2947 & 2 & 42.4 & 1350.585 & -7.5 \\
\hline VBIbXNLGLKU & 87 & 412.4495 & 4 & 42.66 & 1645.795 & -15.8 \\
\hline EAFQMGFTQKU & 87 & 593.2818 & 2 & 43.89 & 1184.565 & -13.2 \\
\hline PLYDJGTGEKU & 87 & 415.5267 & 3 & 44.52 & 1243.579 & -16.6 \\
\hline
\end{tabular}




\begin{tabular}{|c|c|c|c|c|c|c|}
\hline LWPEOSKGKKU & 87 & 319.6807 & 4 & 51.19 & 1274.7 & -4.5 \\
\hline MFEDFBVNFKU & 87 & 484.2203 & 3 & 51.71 & 1449.655 & -11.2 \\
\hline EKYFADZNMKU & 87 & 447.2 & 3 & 54.11 & 1338.603 & -18.2 \\
\hline EKYFGECDMKU & 87 & 670.2972 & 2 & 54.15 & 1338.599 & -14.5 \\
\hline EDLGYQADGKU & 87 & 547.7515 & 2 & 54.4 & 1093.504 & -14.2 \\
\hline GVUQLRIEFKU & 87 & 482.9147 & 3 & 55.18 & 1445.738 & -10.8 \\
\hline TCNDPFFTYKU & 87 & 617.7737 & 2 & 55.96 & 1233.549 & -13 \\
\hline GLBWEYQPFKU & 87 & 361.1819 & 4 & 56.35 & 1440.699 & -0.6 \\
\hline EMGEYAGSiKU & 87 & 602.7598 & 2 & 66.55 & 1203.51 & -4 \\
\hline WUSSYjAEFKU & 87 & 703.3098 & 2 & 67.35 & 1404.618 & -9.4 \\
\hline FMMLKYCFSKU & 87 & 432.8794 & 3 & 68.98 & 1295.623 & -4.9 \\
\hline PTHEFFWELKU & 86 & 333.9173 & 4 & 23.94 & 1331.666 & -19.6 \\
\hline LNSPADVCEKU & 86 & 537.7586 & 2 & 24.48 & 1073.518 & -13.9 \\
\hline GXSLMQMXEKU & 86 & 454.8643 & 3 & 26.89 & 1361.583 & -8.7 \\
\hline URQLFHYEFKU & 86 & 362.4372 & 4 & 28.85 & 1445.732 & -8.2 \\
\hline DMSOAKIMIKU & 86 & 532.5631 & 3 & 29.05 & 1594.684 & -10.1 \\
\hline FOESQDFFHKU & 86 & 463.2067 & 3 & 30.86 & 1386.622 & -16.9 \\
\hline FVKFTCPGNKU & 86 & 570.2979 & 2 & 32.18 & 1138.596 & -12.7 \\
\hline DRWCYEIGVKU & 86 & 482.5495 & 3 & 33.73 & 1444.641 & -9.8 \\
\hline VECTARZVYKU & 86 & 470.9066 & 3 & 33.85 & 1409.712 & -9.7 \\
\hline VCQVFSNPMKU & 86 & 433.8851 & 3 & 35.4 & 1298.651 & -13.1 \\
\hline DDPVVEDKLKU & 86 & 386.2041 & 3 & 36.69 & 1155.614 & -20 \\
\hline SGMDCVCFRKU & 86 & 431.5307 & 3 & 38.32 & 1291.587 & -12.6 \\
\hline PPMGGANVjKU & 86 & 539.7701 & 2 & 38.57 & 1077.536 & -9.4 \\
\hline PYNNWGECRKU & 86 & 471.8884 & 3 & 38.83 & 1412.665 & -15.3 \\
\hline DGQXQTWFGKU & 86 & 429.1984 & 3 & 40.19 & 1284.575 & -1.1 \\
\hline KDDRKjVDMKU & 86 & 448.2313 & 3 & 40.74 & 1341.679 & -5.4 \\
\hline VMGLTDXNLKU & 86 & 605.3067 & 2 & 41.6 & 1208.608 & -7.9 \\
\hline MFEDCYRNFKU & 86 & 451.1982 & 3 & 47.03 & 1350.585 & -8.9 \\
\hline WLIYSDSDKKU & 86 & 477.889 & 3 & 47.6 & 1430.668 & -16 \\
\hline TLKMNLKTGKU & 86 & 378.229 & 3 & 48.04 & 1131.68 & -12.9 \\
\hline FQLFOVAPVKU & 86 & 417.9017 & 3 & 48.34 & 1250.704 & -16.3 \\
\hline FMAMWEjRCKU & 86 & 470.5421 & 3 & 60.94 & 1408.617 & -9.1 \\
\hline FBEFYCWRFKU & 86 & 400.6863 & 4 & 63.44 & 1598.729 & -8.2 \\
\hline PWCWFNKYEKU & 86 & 467.2177 & 3 & 64 & 1398.654 & -16.5 \\
\hline FSjDATEDWKU & 86 & 653.7798 & 2 & 64.83 & 1305.56 & -11.1 \\
\hline TCNDFFFTYKU & 86 & 642.7814 & 2 & 67.01 & 1283.565 & -12.6 \\
\hline NAAGSDTHGKU & 86 & 478.726 & 2 & 71.12 & 955.4471 & -10.2 \\
\hline NEGOJGYDQKU & 86 & 460.5227 & 3 & 76.87 & 1378.572 & -18.6 \\
\hline ETGEWVHcIKU & 86 & 735.3369 & 2 & 78.62 & 1468.667 & -5.2 \\
\hline YDYDCVPTMKU & 86 & 691.3022 & 2 & 92.36 & 1380.608 & -13.4 \\
\hline jLVGQAPRIKU & 85 & 342.6861 & 4 & 24.24 & 1366.729 & -10.3 \\
\hline GPSIbVTVEKU & 85 & 331.9164 & 4 & 28.5 & 1323.649 & -9.6 \\
\hline KTHQXEMMWKU & 85 & 479.8886 & 3 & 29.75 & 1436.655 & -7.8 \\
\hline ZVGESDBFHKU & 85 & 463.2068 & 3 & 30.95 & 1386.612 & -9. \\
\hline
\end{tabular}




\begin{tabular}{|c|c|c|c|c|c|c|}
\hline DDHFADTOQKU & 85 & 427.1839 & 3 & 33.27 & 1278.549 & -14.8 \\
\hline CVLEPCWSAKU & 85 & 641.8127 & 2 & 37 & 1281.624 & -10.3 \\
\hline GVVKQWWOWKU & 85 & 473.9196 & 3 & 37.11 & 1418.747 & -7.2 \\
\hline VGANDSjSMKU & 85 & 558.7521 & 2 & 42.01 & 1115.5 & -9.2 \\
\hline CPFQSTFTQKU & 85 & 593.2819 & 2 & 44.08 & 1184.565 & -13.1 \\
\hline DKVILRAEDKU & 85 & 455.2394 & 3 & 45.54 & 1362.711 & -10.4 \\
\hline bWQMMPjDQKU & 85 & 497.2162 & 3 & 47.24 & 1488.643 & -10.8 \\
\hline FTbGSMQDFKU & 85 & 426.5239 & 3 & 47.99 & 1276.573 & -17.8 \\
\hline FQLRMYAPVKU & 85 & 417.9019 & 3 & 48.42 & 1250.696 & -9.6 \\
\hline PAFjYNUGEKU & 85 & 438.5297 & 3 & 50.05 & 1312.592 & -18.6 \\
\hline XUDETLEQjKU & 85 & 490.8815 & 3 & 50.33 & 1469.637 & -9.4 \\
\hline PDEGAGFTAKU & 85 & 496.2384 & 2 & 50.96 & 990.477 & -14.9 \\
\hline YDLVTSjFCKU & 85 & 642.2972 & 2 & 54.6 & 1282.599 & -14.6 \\
\hline UGVQLRIEFKU & 85 & 482.9146 & 3 & 55.29 & 1445.738 & -11.1 \\
\hline GLBWEYRLCKU & 85 & 361.1818 & 4 & 56.25 & 1440.714 & -10.9 \\
\hline EFFYBNNNEKU & 85 & 493.5451 & 3 & 57.92 & 1477.643 & -19.9 \\
\hline RWMGCRCLYKU & 85 & 488.2416 & 3 & 63.94 & 1461.719 & -10.8 \\
\hline XGSSIECFBKU & 85 & 564.2339 & 3 & 65.84 & 1689.7 & -12.1 \\
\hline MMEMSLSSSKU & 85 & 565.2477 & 2 & 66.71 & 1128.498 & -14.9 \\
\hline NAAGAZSSGKU & 85 & 478.726 & 2 & 71.12 & 955.4471 & -10.2 \\
\hline FOQAVKAAjKU & 85 & 425.5672 & 3 & 73.77 & 1273.676 & 2.7 \\
\hline NBRTCLVFQKU & 85 & 461.5719 & 3 & 77.73 & 1381.709 & -10.9 \\
\hline EMADYQSYIKU & 85 & 684.2884 & 2 & 79.55 & 1366.573 & -7.9 \\
\hline NENDGYVLOKU & 85 & 627.796 & 2 & 80.08 & 1253.59 & -10 \\
\hline SPLVLNACHKU & 85 & 586.3288 & 2 & 83.36 & 1170.659 & -13.6 \\
\hline NDGTRKCQTKU & 84 & 383.8601 & 3 & 24.98 & 1148.572 & -11.7 \\
\hline SYRGWYWORKU & 84 & 376.9377 & 4 & 25.1 & 1503.738 & -11.2 \\
\hline KSMUYVLMSKU & 84 & 422.5512 & 3 & 25.13 & 1264.642 & -8.1 \\
\hline ENSDCYGALKU & 84 & 549.7458 & 2 & 25.85 & 1097.481 & -3.7 \\
\hline ZYPVGEDBHKU & 84 & 471.8783 & 3 & 26.94 & 1412.627 & -10.2 \\
\hline MSVVAHNNEKU & 84 & 376.5224 & 3 & 29.98 & 1126.555 & -8.8 \\
\hline YVGPPGEDQKU & 84 & 544.7659 & 2 & 37 & 1087.53 & -11.6 \\
\hline COYNDJTXGKU & 84 & 527.5525 & 3 & 40.09 & 1579.645 & -5.8 \\
\hline KDDPQFPNSKU & 84 & 392.196 & 3 & 40.43 & 1173.578 & -9.9 \\
\hline VGANADSjMKU & 84 & 550.7546 & 2 & 42.25 & 1099.505 & -9.5 \\
\hline OSEJAVNJSKU & 84 & 735.3207 & 2 & 44.53 & 1468.647 & -13.6 \\
\hline AOLTBVAPVKU & 84 & 319.9303 & 4 & 44.98 & 1275.7 & -6.1 \\
\hline LNDMSCZDRKU & 84 & 425.8555 & 3 & 48.81 & 1274.55 & -3.8 \\
\hline ICCFTLEQjKU & 84 & 490.8817 & 3 & 50.16 & 1469.626 & -1.5 \\
\hline GYDDFMPNSKU & 84 & 586.7479 & 2 & 55.09 & 1171.497 & -13.4 \\
\hline PXYQADYXAKU & 84 & 697.804 & 2 & 56.32 & 1393.602 & -6.4 \\
\hline PGYYQEGYAKU & 84 & 587.7711 & 2 & 56.56 & 1173.545 & -15.1 \\
\hline FUXENCREGKU & 84 & 510.5565 & 3 & 56.75 & 1528.673 & -16.6 \\
\hline LNRILAASDKU & 84 & 407.5594 & 3 & 58.71 & 1219.654 & 1.8 \\
\hline MYGFMTLNMKU & 84 & 412.1919 & 3 & 63.15 & 1233.571 & -13.7 \\
\hline
\end{tabular}




\begin{tabular}{|c|c|c|c|c|c|c|}
\hline ObEFJEGRFKU & 84 & 533.9131 & 3 & 63.44 & 1598.726 & -5.3 \\
\hline LTZFEGESKKU & 84 & 411.5445 & 3 & 63.6 & 1231.62 & -6.4 \\
\hline ijDATDEMSKU & 84 & 669.7747 & 2 & 64.81 & 1337.54 & -3.5 \\
\hline FFAGYLGXGKU & 84 & 589.787 & 2 & 66.55 & 1177.578 & -15.6 \\
\hline FFAGYLEXGKU & 84 & 625.796 & 2 & 68.88 & 1249.599 & -17.3 \\
\hline MWLBbCDFLKU & 84 & 516.5708 & 3 & 69.43 & 1546.708 & -11.4 \\
\hline VFQZMMAQUKU & 84 & 678.8126 & 2 & 69.9 & 1355.623 & -9 \\
\hline FBEMFJWVOKU & 84 & 433.4458 & 4 & 71.33 & 1729.769 & -8.9 \\
\hline TEEGWVHcIKU & 84 & 735.3368 & 2 & 78.54 & 1468.667 & -5.3 \\
\hline WMAYDQNMMKU & 84 & 658.7725 & 2 & 83.05 & 1315.551 & -15.9 \\
\hline FFAXLGENYKU & 84 & 654.3129 & 2 & 85.41 & 1306.621 & -7.1 \\
\hline OQWWGELNMKU & 84 & 697.8178 & 2 & 86.7 & 1393.646 & -18.1 \\
\hline KPRARENUEKU & 83 & 436.236 & 3 & 24.45 & 1305.701 & -11.8 \\
\hline VYGGGHLKIKU & 83 & 397.8807 & 3 & 26.77 & 1190.643 & -18.8 \\
\hline KTHEMWYMWKU & 83 & 480.2219 & 3 & 29.68 & 1437.669 & -17.3 \\
\hline DVAGDJDVNKU & 83 & 399.8501 & 3 & 32.32 & 1196.538 & -7.8 \\
\hline LGSPGAZFEKU & 83 & 367.1843 & 3 & 35.89 & 1098.546 & -13.4 \\
\hline YVGPPPSDNKU & 83 & 536.7686 & 2 & 36.38 & 1071.535 & -11.5 \\
\hline LYMBCVMGRKU & 83 & 458.8859 & 3 & 38.76 & 1373.657 & -15.6 \\
\hline LKXSNGEbjKU & 83 & 474.5638 & 3 & 39.49 & 1420.675 & -3.5 \\
\hline MGGTQYNODKU & 83 & 608.7572 & 2 & 39.5 & 1215.52 & -16.8 \\
\hline SUPDCNQLYKU & 83 & 465.5512 & 3 & 42.29 & 1393.644 & -8.9 \\
\hline MFEDKXGMFKU & 83 & 451.1986 & 3 & 42.3 & 1350.596 & -16.3 \\
\hline PQVPMWGEDKU & 83 & 395.859 & 3 & 49 & 1184.565 & -8 \\
\hline MYGFMTCSTKU & 83 & 389.8316 & 3 & 50.54 & 1166.492 & -16.5 \\
\hline KVKPRBFMGKU & 83 & 455.5948 & 3 & 51.54 & 1363.771 & -6.2 \\
\hline PDWSMQFTAKU & 83 & 403.8556 & 3 & 53.26 & 1208.565 & -16.3 \\
\hline cAYUbVKNQKU & 83 & 481.2384 & 3 & 54.1 & 1440.716 & -15.5 \\
\hline GYDDiCCCSKU & 83 & 613.7068 & 2 & 55.23 & 1225.407 & -6.4 \\
\hline GYDiCCCDSKU & 83 & 613.7066 & 2 & 55.31 & 1225.407 & -6.8 \\
\hline MMAMWEjRCKU & 83 & 465.1995 & 3 & 55.85 & 1392.589 & -9.1 \\
\hline GLBWEYLCRKU & 83 & 481.2405 & 3 & 56.33 & 1440.714 & -9.6 \\
\hline MFITAGEMMKU & 83 & 639.7773 & 2 & 58.66 & 1277.547 & -5.8 \\
\hline UPHLFSAWLKU & 83 & 426.5627 & 3 & 63.12 & 1276.683 & -12.9 \\
\hline iGDDFVLLIKU & 83 & 477.5699 & 3 & 73.55 & 1429.696 & -5.8 \\
\hline SUXEEVQLYKU & 83 & 697.825 & 2 & 73.65 & 1393.649 & -9.6 \\
\hline FBEFFJWVOKU & 83 & 437.4517 & 4 & 75.96 & 1745.797 & -11.1 \\
\hline XbMSMEXjWKU & 83 & 559.8909 & 3 & 91.15 & 1676.662 & -6.8 \\
\hline EMEGYQSWIKU & 83 & 695.7971 & 2 & 91.38 & 1389.589 & -6.6 \\
\hline KPOARAWFLKU & 82 & 330.6939 & 4 & 28.08 & 1318.752 & -4.3 \\
\hline VECSLRAAYKU & 82 & 429.566 & 3 & 28.88 & 1285.684 & -6.4 \\
\hline QAPRQACSGKU & 82 & 596.808 & 2 & 30.01 & 1191.617 & -13.4 \\
\hline STWAKADTRKU & 82 & 388.209 & 3 & 30.09 & 1161.626 & -17.6 \\
\hline SDDCNPYVVKU & 82 & 569.7559 & 2 & 31.7 & 1137.513 & -13.4 \\
\hline LKQQUVHPGKU & 82 & 405.2315 & 3 & 33.31 & 1212.684 & -9.2 \\
\hline
\end{tabular}




\begin{tabular}{|c|c|c|c|c|c|c|}
\hline DDHFAECOQKU & 82 & 432.5153 & 3 & 33.33 & 1294.526 & -1.5 \\
\hline SCTFMSDDKKU & 82 & 387.5017 & 3 & 35.48 & 1159.5 & -14.5 \\
\hline CQFQCVGNNKU & 82 & 479.2253 & 3 & 35.8 & 1434.677 & -16 \\
\hline VPMGAQFNYKU & 82 & 385.1988 & 3 & 37.6 & 1152.575 & -0.4 \\
\hline MKVLTADNFKU & 82 & 389.2113 & 3 & 41.6 & 1164.633 & -17.6 \\
\hline LEMGVTCAGKU & 82 & 336.5065 & 3 & 44.36 & 1006.494 & 3.8 \\
\hline LiPNGVLLBKU & 82 & 454.5872 & 3 & 47.1 & 1360.753 & -10.1 \\
\hline PMAGGNEITKU & 82 & 569.2595 & 2 & 47.4 & 1136.515 & -9.4 \\
\hline TEZFYPEKLKU & 82 & 674.8446 & 2 & 54.48 & 1347.682 & -5.7 \\
\hline YSQMSYAMMKU & 82 & 619.7672 & 2 & 57.47 & 1237.529 & -7.6 \\
\hline CCPLMYVOEKU & 82 & 382.6823 & 4 & 58.17 & 1526.723 & -15.2 \\
\hline YYVNQUDOAKU & 82 & 461.8786 & 3 & 58.61 & 1382.623 & -6.3 \\
\hline YYVGGMLbXKU & 82 & 456.5466 & 3 & 59.41 & 1366.642 & -17.5 \\
\hline LAVLIMYIAKU & 82 & 497.2543 & 3 & 63.4 & 1488.75 & -6.2 \\
\hline QESFOSGEVKU & 82 & 405.1929 & 3 & 71.5 & 1212.564 & -5.4 \\
\hline UPFLFSKNQKU & 82 & 644.3451 & 2 & 87.79 & 1286.688 & -9.8 \\
\hline VPLLVZDOBKU & 82 & 486.2563 & 3 & 92.78 & 1455.754 & -4.5 \\
\hline DWPLLCPXIKU & 82 & 741.3439 & 2 & 106.67 & 1480.689 & -10.2 \\
\hline VSEAQjDMAKU & 81 & 593.7718 & 2 & 24.02 & 1185.542 & -10.7 \\
\hline SGMDTTCOVKU & 81 & 431.5303 & 3 & 25.38 & 1291.579 & -7.8 \\
\hline RCKYcCYNDKU & 81 & 384.1783 & 4 & 25.74 & 1532.698 & -8.6 \\
\hline KVTSCTIZDKU & 81 & 437.2039 & 3 & 26.53 & 1308.6 & -7.7 \\
\hline TGQGGGWYiKU & 81 & 396.1846 & 3 & 28.09 & 1185.544 & -9.6 \\
\hline EMDKSQAPDKU & 81 & 383.1788 & 3 & 30.29 & 1146.534 & -17 \\
\hline XDGVLKVGEKU & 81 & 388.5445 & 3 & 35.9 & 1162.621 & -7.6 \\
\hline LAAKQWWOWKU & 81 & 473.9196 & 3 & 37.11 & 1418.747 & -7.2 \\
\hline PPEODCSSGKU & 81 & 561.7318 & 2 & 38.39 & 1121.467 & -16.1 \\
\hline VTCLTDNIVKU & 81 & 409.2038 & 3 & 41.51 & 1224.604 & -11.8 \\
\hline KVIEMQLQVKU & 81 & 464.9149 & 3 & 44.18 & 1391.745 & -15.6 \\
\hline NMFLMKXVVKU & 81 & 443.5692 & 3 & 46.34 & 1327.7 & -11.1 \\
\hline CMLSZFDNVKU & 81 & 417.5236 & 3 & 46.38 & 1249.558 & -7.6 \\
\hline PTYAETTMSKU & 81 & 564.2657 & 2 & 48.44 & 1126.533 & -14.2 \\
\hline MAMWEjTSAKU & 81 & 631.7783 & 2 & 48.83 & 1261.555 & -10.7 \\
\hline PARPMEGWDKU & 81 & 395.859 & 3 & 49 & 1184.576 & -17.5 \\
\hline MYAQPMGQFKU & 81 & 600.2808 & 2 & 49.34 & 1198.563 & -13.2 \\
\hline AGQOGYjLcKU & 81 & 671.8226 & 2 & 52.28 & 1341.638 & -5.3 \\
\hline LBjPMMYYQKU & 81 & 519.5757 & 3 & 60.82 & 1555.709 & -2.3 \\
\hline FFAGYLAXGKU & 81 & 596.795 & 2 & 68.5 & 1191.594 & -15.3 \\
\hline MMFLKYYACKU & 81 & 432.8793 & 3 & 69.05 & 1295.623 & -5.3 \\
\hline NYETTYDMVKU & 81 & 631.778 & 2 & 71.38 & 1261.565 & -18.7 \\
\hline EGMEMSLOGKU & 81 & 592.7656 & 2 & 78.17 & 1183.523 & -5.1 \\
\hline KWWMSCPWWKU & 81 & 479.5633 & 3 & 81.69 & 1435.668 & -0.1 \\
\hline IRQQMGFVDKU & 81 & 699.8371 & 2 & 84.59 & 1397.673 & -9.3 \\
\hline PTHEFMILPKU & 80 & 333.9173 & 4 & 23.94 & 1331.656 & -12 \\
\hline jKVMDGYQGKU & 80 & 411.868 & 3 & 24.12 & 1232.594 & -9.8 \\
\hline
\end{tabular}




\begin{tabular}{|c|c|c|c|c|c|c|}
\hline KQEKIVEAGKU & 80 & 436.2352 & 3 & 24.57 & 1305.689 & -4.1 \\
\hline FGQQLQEMMKU & 80 & 413.5316 & 3 & 27.29 & 1237.595 & -17.5 \\
\hline DMSOAGAFIKU & 80 & 440.8618 & 3 & 27.38 & 1319.568 & -3.4 \\
\hline XNGUPLMTHKU & 80 & 432.871 & 3 & 28.59 & 1295.605 & -10.9 \\
\hline VGANAYECYKU & 80 & 558.7505 & 2 & 30.42 & 1115.507 & -18.2 \\
\hline VLCFAAFSYKU & 80 & 324.6733 & 4 & 31.65 & 1294.678 & -10.3 \\
\hline DQYYDAHWKKU & 80 & 451.5483 & 3 & 32.33 & 1351.631 & -5.8 \\
\hline KMESAKEјOKU & 80 & 341.4206 & 4 & 32.49 & 1361.659 & -4.3 \\
\hline QOASDRRFGKU & 80 & 423.219 & 3 & 32.49 & 1266.644 & -7 \\
\hline VDOYCFNSMKU & 80 & 365.1611 & 4 & 33.42 & 1456.637 & -15 \\
\hline KALNPMMNLKU & 80 & 290.415 & 4 & 34.2 & 1157.641 & -9.1 \\
\hline GTQDASKGKKU & 80 & 340.19 & 3 & 36.58 & 1017.557 & -8.4 \\
\hline RWILPGSVMKU & 80 & 455.2401 & 3 & 38.45 & 1362.708 & -7.1 \\
\hline GGMPNVLLVKU & 80 & 342.8689 & 3 & 39.79 & 1025.606 & -20 \\
\hline ESCTWYTKXKU & 80 & 455.5405 & 3 & 42.49 & 1363.609 & -7 \\
\hline YASYMMASEKU & 80 & 590.2526 & 2 & 46.58 & 1178.51 & -16.5 \\
\hline PAGVDEZjDKU & 80 & 411.8525 & 3 & 49.05 & 1232.539 & -3 \\
\hline LNWPMDZVAKU & 80 & 423.2083 & 3 & 52.36 & 1266.618 & -11.8 \\
\hline MVAFMERTLKU & 80 & 408.8887 & 3 & 52.67 & 1223.652 & -6.1 \\
\hline DQYYWHNKKKU & 80 & 470.2354 & 3 & 52.78 & 1407.705 & -14.6 \\
\hline VcEQDSUVYKU & 80 & 670.8118 & 2 & 54.74 & 1339.624 & -10.9 \\
\hline VcETDYMVYKU & 80 & 670.8118 & 2 & 54.74 & 1339.62 & -8.1 \\
\hline PXYGAADYAKU & 80 & 587.7712 & 2 & 56.47 & 1173.532 & -3.1 \\
\hline EDQGEJVFQKU & 80 & 672.8 & 2 & 61.72 & 1343.606 & -15.4 \\
\hline XADDFVLLQKU & 80 & 423.216 & 3 & 65.07 & 1266.647 & -16.2 \\
\hline bMGMEHQjbKU & 80 & 502.2166 & 3 & 65.16 & 1503.635 & -4.8 \\
\hline ACGVQWIDLKU & 80 & 437.2103 & 3 & 66.93 & 1308.614 & -3.7 \\
\hline VCYVMZbEDKU & 80 & 699.8027 & 2 & 71 & 1397.592 & -1 \\
\hline NWYZDGGGFKU & 80 & 413.1811 & 3 & 71.84 & 1236.531 & -8 \\
\hline PYRYFQNSSKU & 79 & 322.9151 & 4 & 24.33 & 1287.636 & -3.7 \\
\hline LEHALLRAHKU & 79 & 297.4313 & 4 & 24.39 & 1185.71 & -11.2 \\
\hline EGbTFAVNKKU & 79 & 404.2153 & 3 & 25.74 & 1209.632 & -6.6 \\
\hline UFCDHAFVGKU & 79 & 401.5199 & 3 & 28.31 & 1201.545 & -6.2 \\
\hline KEXVNYDYEKU & 79 & 469.5514 & 3 & 28.33 & 1405.638 & -3.7 \\
\hline GAAPELLEKU & 79 & 398.2061 & 3 & 30.18 & 1191.62 & -19.9 \\
\hline PICPFNSYTKU & 79 & 479.8911 & 3 & 34.54 & 1436.666 & -9.8 \\
\hline MGMјEAbKMKU & 79 & 451.2035 & 3 & 34.69 & 1350.603 & -10.8 \\
\hline XFXIVRTGDKU & 79 & 499.2296 & 3 & 35.3 & 1494.684 & -11.6 \\
\hline MGLOcNSADKU & 79 & 411.5202 & 3 & 37.39 & 1231.56 & -16.8 \\
\hline NMPGGVLLVKU & 79 & 342.869 & 3 & 39.69 & 1025.606 & -19.6 \\
\hline PTFEFWEFLKU & 79 & 448.2239 & 3 & 48.07 & 1341.676 & -19.2 \\
\hline PAMjFVDADKU & 79 & 401.1906 & 3 & 49.24 & 1200.557 & -5.6 \\
\hline TLPKFNKTGKU & 79 & 378.2296 & 3 & 49.27 & 1131.676 & -8.1 \\
\hline LLKVAGYXbKU & 79 & 443.5789 & 3 & 50.3 & 1327.733 & -13.7 \\
\hline QKEFBEWEQKU & 79 & 382.1794 & 4 & 50.55 & 1524.716 & -18 \\
\hline
\end{tabular}




\begin{tabular}{|c|c|c|c|c|c|c|}
\hline LWUQQEQGKKU & 79 & 441.8997 & 3 & 51.09 & 1322.684 & -5.2 \\
\hline ZDDNABFMGKU & 79 & 456.1907 & 3 & 51.61 & 1365.557 & -5.3 \\
\hline EGMYQRcYYKU & 79 & 477.5591 & 3 & 52.4 & 1429.653 & 1.8 \\
\hline CIFCVELAGKU & 79 & 481.2392 & 3 & 53.88 & 1440.705 & -6.4 \\
\hline EKYFCMFNMKU & 79 & 447.2 & 3 & 54.11 & 1338.592 & -10.6 \\
\hline SMHQUDFTYKU & 79 & 445.8638 & 3 & 54.43 & 1334.583 & -9.7 \\
\hline VcPCGCMVYKU & 79 & 670.812 & 2 & 55.01 & 1339.62 & -7.9 \\
\hline FKDTYKSSVKU & 79 & 401.2174 & 3 & 58.4 & 1200.65 & -16.4 \\
\hline SMEMSLSSSKU & 79 & 543.2441 & 2 & 59.99 & 1084.489 & -14.4 \\
\hline WCTLNAYGEKU & 79 & 592.2823 & 2 & 65.24 & 1182.549 & 0.9 \\
\hline TVSWIZjVVKU & 79 & 504.9136 & 3 & 68.4 & 1511.734 & -10.1 \\
\hline ADASFXQEVKU & 79 & 607.2866 & 2 & 71.58 & 1212.564 & -4 \\
\hline YFKYACSbWKU & 79 & 706.8279 & 2 & 72.82 & 1411.656 & -10.6 \\
\hline bCFLSVMOGKU & 79 & 435.8759 & 3 & 73.78 & 1304.609 & -2 \\
\hline DSVFLYZMTKU & 79 & 649.3088 & 2 & 77.61 & 1296.617 & -10.8 \\
\hline TZFLSVYDNKU & 79 & 640.8077 & 2 & 81.22 & 1279.62 & -14.7 \\
\hline ZYPVGENOjKU & 78 & 471.8781 & 3 & 26.54 & 1412.63 & -12.7 \\
\hline KYbCSQQJYKU & 78 & 510.8956 & 3 & 26.96 & 1529.686 & -13.6 \\
\hline TDOQQFELYKU & 78 & 458.884 & 3 & 27.86 & 1373.648 & -12.5 \\
\hline TCKUQLMPSKU & 78 & 454.9005 & 3 & 29.09 & 1361.694 & -10.3 \\
\hline LETJFLDTNKU & 78 & 449.2235 & 3 & 30.97 & 1344.663 & -10.5 \\
\hline PNLBMSGGTKU & 78 & 393.5258 & 3 & 31.49 & 1177.572 & -13.5 \\
\hline YKYEGTIWHKU & 78 & 361.9221 & 4 & 33.43 & 1443.68 & -14.6 \\
\hline ZTVVHXWODKU & 78 & 501.5598 & 3 & 33.43 & 1501.682 & -16.5 \\
\hline YNCMVALBRKU & 78 & 457.8925 & 3 & 33.66 & 1370.675 & -14.3 \\
\hline KLNAPMMNLKU & 78 & 290.415 & 4 & 34.09 & 1157.641 & -9.1 \\
\hline STLDSPHMEKU & 78 & 572.2679 & 2 & 35.11 & 1142.539 & -15.5 \\
\hline KGbcLCFAGKU & 78 & 346.4296 & 4 & 35.19 & 1381.71 & -15.1 \\
\hline PPERCCDSGKU & 78 & 545.7374 & 2 & 38.42 & 1089.47 & -8.5 \\
\hline PDLGGANUQKU & 78 & 539.77 & 2 & 38.82 & 1077.532 & -5.6 \\
\hline KjNWRGbjMKU & 78 & 389.4374 & 4 & 39.69 & 1553.743 & -14.5 \\
\hline CGTTQYNODKU & 78 & 616.7542 & 2 & 39.8 & 1231.515 & -17.4 \\
\hline ASCTQYNODKU & 78 & 616.7538 & 2 & 39.91 & 1231.515 & -17.9 \\
\hline KQObFGYNQKU & 78 & 478.5664 & 3 & 41.96 & 1432.693 & -10.8 \\
\hline PAEDRWGEDKU & 78 & 401.1896 & 3 & 43.22 & 1200.552 & -4.4 \\
\hline EGGEXNMSGKU & 78 & 564.2346 & 2 & 43.99 & 1126.457 & -2.3 \\
\hline LNFBEQLQVKU & 78 & 348.9379 & 4 & 44.29 & 1391.736 & -9.9 \\
\hline CVQMCAFFDKU & 78 & 669.7983 & 2 & 46.57 & 1337.596 & -10.5 \\
\hline OXRPDDjDQKU & 78 & 502.5481 & 3 & 47.39 & 1504.639 & -10.7 \\
\hline GASEMICVFKU & 78 & 470.5439 & 3 & 48.16 & 1408.636 & -18.6 \\
\hline UNVLSRTIDKU & 78 & 468.2346 & 3 & 48.56 & 1401.697 & -10.4 \\
\hline jMMSGZSDLKU & 78 & 424.5169 & 3 & 49.97 & 1270.541 & -9.2 \\
\hline EGMYQYRcYKU & 78 & 477.5594 & 3 & 52.3 & 1429.653 & 2.4 \\
\hline KAGESCiLGKU & 78 & 563.2762 & 2 & 52.91 & 1124.552 & -12.2 \\
\hline EjCPSLWOCKU & 78 & 459.8679 & 3 & 53.18 & 1376.584 & -1.4 \\
\hline
\end{tabular}




\begin{tabular}{|c|c|c|c|c|c|c|}
\hline KYYDDCMVYKU & 78 & 492.2207 & 3 & 54.82 & 1473.666 & -17.6 \\
\hline YNHLFSKNQKU & 78 & 426.5627 & 3 & 63.12 & 1276.668 & -1 \\
\hline EGRMXiLLYKU & 78 & 488.2408 & 3 & 63.83 & 1461.717 & -11 \\
\hline OGMYFCQCFKU & 78 & 784.8489 & 2 & 66.93 & 1567.692 & -5.8 \\
\hline OQADGELNMKU & 78 & 604.7818 & 2 & 68.11 & 1207.552 & -2 \\
\hline NNNFADESKKU & 78 & 583.2761 & 2 & 69.46 & 1164.552 & -12.5 \\
\hline WGUPCGFGQKU & 78 & 579.7596 & 2 & 74.54 & 1157.519 & -12.3 \\
\hline NDGTRVMMVKU & 78 & 575.2874 & 2 & 75.08 & 1148.579 & -16.6 \\
\hline AYNDASDLMKU & 78 & 563.7549 & 2 & 75.16 & 1125.513 & -15.2 \\
\hline ICSCTAYQVKU & 78 & 618.2656 & 2 & 87.72 & 1234.534 & -14.3 \\
\hline VPLLVcNEbKU & 78 & 661.8631 & 2 & 92.96 & 1321.729 & -13.1 \\
\hline ODSGSYXAGKU & 78 & 604.2567 & 2 & 101.14 & 1206.503 & -3.2 \\
\hline MCYRGJDRDKU & 77 & 389.9282 & 4 & 25.48 & 1555.697 & -8.6 \\
\hline GJQPKCVGNKU & 77 & 569.7855 & 2 & 30.28 & 1137.568 & -10.4 \\
\hline DPSXNYSEVKU & 77 & 419.8554 & 3 & 33.07 & 1256.553 & -7 \\
\hline STLDSHMPEKU & 77 & 572.2678 & 2 & 34.95 & 1142.539 & -15.7 \\
\hline STLDSUGQEKU & 77 & 572.2681 & 2 & 35.02 & 1142.532 & -8.7 \\
\hline MYEDCYRNFKU & 77 & 456.5294 & 3 & 35.97 & 1366.58 & -9.7 \\
\hline LNYMPDWHDKU & 77 & 439.8701 & 3 & 37.97 & 1316.597 & -6.5 \\
\hline FAPZJAGHZKU & 77 & 461.5453 & 3 & 38.86 & 1381.623 & -6.8 \\
\hline MPVSUGDPJKU & 77 & 638.2935 & 2 & 39.16 & 1274.578 & -4.5 \\
\hline PWZCSPKENKU & 77 & 428.2019 & 3 & 42.87 & 1281.593 & -6.7 \\
\hline NMFLMKLbSKU & 77 & 443.5696 & 3 & 46.46 & 1327.696 & -6.7 \\
\hline iFPMSYAMMKU & 77 & 446.867 & 3 & 46.57 & 1337.584 & -3.3 \\
\hline TNECOMCVFKU & 77 & 475.8752 & 3 & 48.21 & 1424.614 & -7.3 \\
\hline EDAGADJFQKU & 77 & 623.2685 & 2 & 50.06 & 1244.538 & -12.4 \\
\hline jMMSGYAFEKU & 77 & 636.2743 & 2 & 50.1 & 1270.544 & -8.2 \\
\hline FMVVBPIWOKU & 77 & 419.699 & 4 & 50.67 & 1674.79 & -13.6 \\
\hline OEGMYWAQWKU & 77 & 467.8789 & 3 & 52.13 & 1400.62 & -3.4 \\
\hline EjPCSLWCOKU & 77 & 459.8667 & 3 & 53.27 & 1376.584 & -3.9 \\
\hline HHLSZFDVNKU & 77 & 430.8796 & 3 & 55.43 & 1289.627 & -7.3 \\
\hline YDAYTQEMMKU & 77 & 639.7772 & 2 & 58.76 & 1277.542 & -1.6 \\
\hline VPLLVWATCKU & 77 & 376.8858 & 3 & 61.42 & 1127.653 & -15 \\
\hline CILTSTCPTKU & 77 & 696.3297 & 2 & 64.59 & 1390.647 & -1.3 \\
\hline AYECACAFQKU & 77 & 612.2767 & 2 & 66.97 & 1222.552 & -10.7 \\
\hline MRADPESXJKU & 77 & 473.5457 & 3 & 69.84 & 1417.623 & -5 \\
\hline GGADYXOGFKU & 77 & 413.1811 & 3 & 71.77 & 1236.528 & -5.5 \\
\hline KCUPYSKLEKU & 77 & 425.5551 & 3 & 75.06 & 1273.66 & -13.1 \\
\hline ZSLGADEXcKU & 77 & 664.2933 & 2 & 83.4 & 1326.578 & -4.6 \\
\hline AUAYGEMMDKU & 77 & 597.7453 & 2 & 88.62 & 1193.496 & -16.6 \\
\hline ZSLGADEIUKU & 77 & 664.2943 & 2 & 95.32 & 1326.582 & -6.1 \\
\hline QNHALLKVHKU & 76 & 297.4313 & 4 & 24.39 & 1185.71 & -11.2 \\
\hline LNSPADCDLKU & 76 & 537.7581 & 2 & 24.6 & 1073.518 & -14.8 \\
\hline PSGSZGMAGKU & 76 & 493.2245 & 2 & 25.33 & 984.4447 & -10.4 \\
\hline QTMAGGQCUKU & 76 & 597.2722 & 2 & 27.53 & 1192.549 & -15.8 \\
\hline
\end{tabular}




\begin{tabular}{|c|c|c|c|c|c|c|}
\hline KPFQRAWFLKU & 76 & 330.6942 & 4 & 27.99 & 1318.766 & -14 \\
\hline KbKCRQYEFKU & 76 & 362.4371 & 4 & 28.95 & 1445.742 & -15.6 \\
\hline PNLAOQMGTKU & 76 & 388.1942 & 3 & 31.43 & 1161.583 & -18.8 \\
\hline TYVLEHMDQKU & 76 & 421.5389 & 3 & 32.06 & 1261.613 & -14.1 \\
\hline KGMRRDSiGKU & 76 & 423.2188 & 3 & 32.38 & 1266.648 & -10.9 \\
\hline NYGDPQQHNKU & 76 & 600.2761 & 2 & 38.11 & 1198.548 & -8.5 \\
\hline YjKCQjYDMKU & 76 & 748.3107 & 2 & 39.11 & 1494.633 & -17.2 \\
\hline MNTGAYNODKU & 76 & 608.7574 & 2 & 39.65 & 1215.52 & -16.5 \\
\hline DGQCPTWFGKU & 76 & 429.1972 & 3 & 39.98 & 1284.595 & -19.9 \\
\hline DGQcGPTWOKU & 76 & 429.1983 & 3 & 40.09 & 1284.583 & -7.5 \\
\hline PWZCSLQWGKU & 76 & 433.5337 & 3 & 42.72 & 1297.603 & -18 \\
\hline CUCMTWYTCKU & 76 & 704.7883 & 2 & 44.46 & 1407.56 & 1.3 \\
\hline PLYDWHCSPKU & 76 & 415.5272 & 3 & 44.88 & 1243.581 & -16.9 \\
\hline LYCDKMFiNKU & 76 & 771.8685 & 2 & 45.05 & 1541.727 & -2.9 \\
\hline KIKNCVAPVKU & 76 & 319.9301 & 4 & 45.09 & 1275.697 & -4.7 \\
\hline CVAGMSiFDKU & 76 & 669.7983 & 2 & 46.57 & 1337.601 & -13.8 \\
\hline QbWMMPjDQKU & 76 & 497.217 & 3 & 47.42 & 1488.643 & -9.1 \\
\hline HQDGCUFNFKU & 76 & 637.7719 & 2 & 48.9 & 1273.541 & -9.2 \\
\hline MMAEANCEKKU & 76 & 434.5371 & 3 & 49.5 & 1300.597 & -5.8 \\
\hline LNJWQEWEQKU & 76 & 509.2381 & 3 & 50.76 & 1524.707 & -9.2 \\
\hline MMAMWEIYAKU & 76 & 465.1995 & 3 & 55.85 & 1392.59 & -9.2 \\
\hline YLGAFPDSSKU & 76 & 542.2711 & 2 & 59.03 & 1082.54 & -11.1 \\
\hline PEDbGINFMKU & 76 & 482.5361 & 3 & 59.64 & 1444.611 & -17.2 \\
\hline EDQADJVOAKU & 76 & 672.8 & 2 & 61.67 & 1343.592 & -5.1 \\
\hline SPLVLNAGEKU & 76 & 513.7935 & 2 & 64.28 & 1025.587 & -14.1 \\
\hline BMMMVLLGAKU & 76 & 634.3214 & 2 & 65.09 & 1266.645 & -13.4 \\
\hline MDEMSLSSSKU & 76 & 557.2407 & 2 & 66.33 & 1112.484 & -15.5 \\
\hline RVEONGGLFKU & 76 & 611.8251 & 2 & 68.09 & 1221.648 & -10.1 \\
\hline MMFLKMZSSKU & 76 & 648.8163 & 2 & 69.15 & 1295.619 & -0.6 \\
\hline NWYTCYGGFKU & 76 & 413.1811 & 3 & 71.85 & 1236.539 & -13.9 \\
\hline XSDDFVLLIKU & 76 & 482.9008 & 3 & 73.81 & 1445.69 & -6.7 \\
\hline MOPWLFEXDKU & 76 & 496.8865 & 3 & 75.52 & 1487.663 & -17 \\
\hline ZMMEMSLOGKU & 76 & 662.7872 & 2 & 75.97 & 1323.564 & -2.7 \\
\hline YcLDJEGGXKU & 76 & 730.8116 & 2 & 79.52 & 1459.627 & -12.1 \\
\hline NKHPSLKVHKU & 75 & 297.4313 & 4 & 24.53 & 1185.71 & -11.4 \\
\hline QTMANQWGMKU & 75 & 597.2737 & 2 & 27.84 & 1192.548 & -12.7 \\
\hline QPRANLLCQKU & 75 & 315.9331 & 4 & 28.19 & 1259.718 & -11.6 \\
\hline FYASMXNATKU & 75 & 417.8536 & 3 & 29.11 & 1250.561 & -17.7 \\
\hline HSKVCKJMVKU & 75 & 491.5926 & 3 & 31.02 & 1471.774 & -12.2 \\
\hline CEWFHCYNDKU & 75 & 497.8743 & 3 & 31.87 & 1490.61 & -6.1 \\
\hline MTLAERFAHKU & 75 & 401.549 & 3 & 32.07 & 1201.639 & -11.6 \\
\hline KSMLDNFELKU & 75 & 408.5478 & 3 & 32.76 & 1222.638 & -13.4 \\
\hline DPTVPSPMGKU & 75 & 514.2587 & 2 & 33.67 & 1026.517 & -13.7 \\
\hline AXPVOYMADKU & 75 & 439.8676 & 3 & 36.37 & 1316.595 & -10.4 \\
\hline VGYPTDNIVKU & 75 & 409.2041 & 3 & 41.61 & 1224.601 & -8.3 \\
\hline
\end{tabular}




\begin{tabular}{|c|c|c|c|c|c|c|}
\hline LTVGUBXNQKU & 75 & 478.566 & 3 & 41.82 & 1432.687 & -7.7 \\
\hline VQNAUGCAMKU & 75 & 550.7548 & 2 & 42.14 & 1099.502 & -5.8 \\
\hline FLFDCSYEOKU & 75 & 452.1985 & 3 & 46.72 & 1353.592 & -13.8 \\
\hline LNSAALEZNKU & 75 & 385.1995 & 3 & 47.01 & 1152.589 & -10.3 \\
\hline WSYTHGITKKU & 75 & 447.5536 & 3 & 49.77 & 1339.654 & -11.3 \\
\hline NEMQNQFTAKU & 75 & 403.8564 & 3 & 53.38 & 1208.561 & -11.1 \\
\hline JScEGELPDKU & 75 & 667.2971 & 2 & 53.54 & 1332.598 & -13.9 \\
\hline YLTGPFSDGKU & 75 & 542.2712 & 2 & 58.94 & 1082.54 & -10.8 \\
\hline QWcWOQDFVKU & 75 & 511.9091 & 3 & 62.53 & 1532.714 & -5.5 \\
\hline MYGFMTTWAKU & 75 & 412.1912 & 3 & 63.29 & 1233.567 & -12.7 \\
\hline ZCMPYELKIKU & 75 & 748.8423 & 2 & 63.96 & 1495.68 & -7 \\
\hline GQKYNjOMFKU & 75 & 476.5541 & 3 & 64.88 & 1426.665 & -16.9 \\
\hline ijDATECETKU & 75 & 669.7741 & 2 & 64.99 & 1337.54 & -4.4 \\
\hline EEDOSGKbWKU & 75 & 467.2103 & 3 & 65.5 & 1398.625 & -11 \\
\hline KUOCCSQYYKU & 75 & 469.1957 & 3 & 67.6 & 1404.593 & -19.5 \\
\hline jTKYASCbWKU & 75 & 706.8268 & 2 & 73 & 1411.649 & -7.4 \\
\hline MWLSGbMMLKU & 75 & 438.5442 & 3 & 74.3 & 1312.631 & -15.6 \\
\hline ZCLPYSKLEKU & 75 & 425.5552 & 3 & 74.88 & 1273.649 & -4 \\
\hline YbPQSTFVDKU & 75 & 651.3109 & 2 & 77.28 & 1300.627 & -14.9 \\
\hline MAGELGYQDKU & 75 & 555.7639 & 2 & 77.49 & 1109.518 & -3.9 \\
\hline MAEGLGYNEKU & 75 & 555.7655 & 2 & 77.69 & 1109.518 & -1 \\
\hline XTLKOHAIYKU & 75 & 506.5831 & 3 & 88.93 & 1516.742 & -9.3 \\
\hline SKCDSVMFNKU & 75 & 435.8761 & 3 & 91 & 1304.625 & -13.9 \\
\hline DOSGSYGIGKU & 75 & 604.2564 & 2 & 101.09 & 1206.503 & -4.1 \\
\hline VSEAQjCDVKU & 74 & 593.7697 & 2 & 24.17 & 1185.542 & -14.3 \\
\hline KVTCTSiZDKU & 74 & 437.2044 & 3 & 26.3 & 1308.6 & -6.5 \\
\hline TITFAGPGVKU & 74 & 584.2991 & 2 & 26.93 & 1166.594 & -8.4 \\
\hline SSAAPPCQVKU & 74 & 493.7525 & 2 & 27.27 & 985.5015 & -11.2 \\
\hline TDZDQVNAAKU & 74 & 385.8475 & 3 & 27.5 & 1154.532 & -9.3 \\
\hline MSVVANANZKU & 74 & 564.28 & 2 & 30.53 & 1126.555 & -8.8 \\
\hline KGRZXLDTNKU & 74 & 449.2242 & 3 & 31.19 & 1344.676 & -18.6 \\
\hline KNKbFDTGGKU & 74 & 404.5463 & 3 & 31.2 & 1210.627 & -8.5 \\
\hline VEKSCAFWAKU & 74 & 584.295 & 2 & 33.31 & 1166.591 & -13 \\
\hline YTNCZHQWEKU & 74 & 468.198 & 3 & 33.94 & 1401.588 & -11.5 \\
\hline MLVENGPVGKU & 74 & 348.1889 & 3 & 34.19 & 1041.564 & -18.3 \\
\hline DDHFMSSWQKU & 74 & 427.1833 & 3 & 34.65 & 1278.545 & -13.3 \\
\hline KGDCCZLCcKU & 74 & 469.2015 & 3 & 34.82 & 1404.606 & -16.6 \\
\hline DASbLKVGEKU & 74 & 388.5443 & 3 & 35.63 & 1162.616 & -4.5 \\
\hline jTHVAEAJYKU & 74 & 348.918 & 4 & 36.41 & 1391.651 & -5.8 \\
\hline DDPVVEKMPKU & 74 & 386.2041 & 3 & 36.68 & 1155.596 & -4.6 \\
\hline CVLUDGSYAKU & 74 & 641.8123 & 2 & 37.06 & 1281.617 & -5.2 \\
\hline LKXSNWSMjKU & 74 & 474.5637 & 3 & 39.4 & 1420.675 & -4.2 \\
\hline DMOVVAGFTKU & 74 & 390.8663 & 3 & 41.25 & 1169.576 & 0.6 \\
\hline HKACIJSSHKU & 74 & 466.2148 & 3 & 44.55 & 1395.629 & -4.6 \\
\hline FQLRDQFPVKU & 74 & 426.2386 & 3 & 44.95 & 1275.709 & -11 \\
\hline
\end{tabular}




\begin{tabular}{|c|c|c|c|c|c|c|}
\hline PWJSPKGEGKU & 74 & 417.5389 & 3 & 45.44 & 1249.616 & -16.9 \\
\hline YEUQWDMLSKU & 74 & 460.209 & 3 & 46.25 & 1377.614 & -6 \\
\hline TNEMIGcVFKU & 74 & 470.5446 & 3 & 47.88 & 1408.638 & -18.4 \\
\hline LNMFVRTGDKU & 74 & 393.8748 & 3 & 48.33 & 1178.623 & -17.3 \\
\hline FQFVLOAPVKU & 74 & 417.9017 & 3 & 48.34 & 1250.704 & -16.3 \\
\hline jMMSGYEAFKU & 74 & 424.5179 & 3 & 50.07 & 1270.544 & -9.9 \\
\hline VGAMCFAFDKU & 74 & 544.2488 & 2 & 50.6 & 1086.499 & -14.6 \\
\hline PMAMWECHHKU & 74 & 453.8703 & 3 & 51.71 & 1358.609 & -14.8 \\
\hline YSADTKMPMKU & 74 & 390.8578 & 3 & 52.63 & 1169.557 & -4.8 \\
\hline MKSYKHSYFKU & 74 & 439.8894 & 3 & 62.89 & 1316.67 & -17.9 \\
\hline ijDATMDSPKU & 74 & 653.7788 & 2 & 65.08 & 1305.55 & - \\
\hline bMGMEHFHHKU & 74 & 685.8054 & 2 & 65.21 & 1369.599 & -1.9 \\
\hline ONDUGGFTYKU & 74 & 642.7814 & 2 & 67.02 & 1283.554 & -4.8 \\
\hline SSGVQQANXKU & 74 & 569.2714 & 2 & 68 & 1136.544 & -13.4 \\
\hline PCDFCiLPVKU & 74 & 498.5862 & 3 & 73.22 & 1492.736 & 0.5 \\
\hline ZKEESVMFNKU & 74 & 435.8759 & 3 & 74.94 & 1304.618 & -9.6 \\
\hline NDGTRQKCTKU & 74 & 575.2874 & 2 & 75.08 & 1148.572 & -10.2 \\
\hline DMCSAKEJFKU & 74 & 491.2183 & 3 & 75.73 & 1470.658 & -17 \\
\hline NEGODDHIQKU & 74 & 460.5233 & 3 & 76.98 & 1378.563 & -10.8 \\
\hline jDELWLDEGKU & 74 & 656.8012 & 2 & 86.59 & 1311.606 & -14.2 \\
\hline UPFLQHPLEKU & 74 & 644.3453 & 2 & 87.73 & 1286.688 & -9.4 \\
\hline KiLIHAFSYKU & 74 & 506.5826 & 3 & 89.03 & 1516.755 & -18.9 \\
\hline FMADALGWXKU & 74 & 629.2974 & 2 & 90.76 & 1256.587 & -5.6 \\
\hline VQCLLVcWBKU & 74 & 486.2563 & 3 & 92.7 & 1455.758 & -7.3 \\
\hline PTHEFMHcLKU & 73 & 333.9179 & 4 & 24.23 & 1331.652 & -7.3 \\
\hline KIHEENAKSKU & 73 & 454.226 & 3 & 25.36 & 1359.675 & -13.6 \\
\hline ELLTFCDMTKU & 73 & 400.5243 & 3 & 25.41 & 1198.573 & -18 \\
\hline ENSDPGCHLKU & 73 & 549.747 & 2 & 25.63 & 1097.492 & -11.8 \\
\hline LYMKYCJNDKU & 73 & 384.1786 & 4 & 25.88 & 1532.712 & -17.2 \\
\hline KYBCTGQJYKU & 73 & 383.4232 & 4 & 26.93 & 1529.684 & -13.4 \\
\hline GVLPEENIWKU & 73 & 435.5531 & 3 & 29.82 & 1303.643 & -4.2 \\
\hline EMDELTPGEKU & 73 & 574.2639 & 2 & 30.4 & 1146.523 & -8.3 \\
\hline TNFOYCANjKU & 73 & 458.1994 & 3 & 32.39 & 1371.586 & -7.1 \\
\hline DDHFWTCTQKU & 73 & 427.1839 & 3 & 33.16 & 1278.545 & -11.9 \\
\hline LNGMSIDYEKU & 73 & 645.2869 & 2 & 36.36 & 1288.563 & -2.5 \\
\hline NATGESKGKKU & 73 & 340.1901 & 3 & 36.69 & 1017.557 & -8.1 \\
\hline MXFMTSSCAKU & 73 & 408.8392 & 3 & 37.85 & 1223.5 & -3.2 \\
\hline KADBLEVGLKU & 73 & 416.2349 & 3 & 37.87 & 1245.688 & -4.4 \\
\hline MYOMTASCSKU & 73 & 408.8387 & 3 & 38.04 & 1223.5 & -4.4 \\
\hline GEFRTHQWMKU & 73 & 440.2175 & 3 & 39.3 & 1317.64 & -7.2 \\
\hline MNTQUTPCDKU & 73 & 608.7569 & 2 & 39.74 & 1215.513 & -10.9 \\
\hline MKVLTDGQFKU & 73 & 389.2113 & 3 & 41.6 & 1164.633 & -17.6 \\
\hline CVYCYZNZKKU & 73 & 519.9015 & 3 & 49.1 & 1556.69 & -4.4 \\
\hline ZOQLEQTCFKU & 73 & 465.5481 & 3 & 51.12 & 1393.631 & - \\
\hline XDDFMPGGSKU & 73 & 586.7481 & 2 & 55.17 & 1171.483 & -1 \\
\hline
\end{tabular}




\begin{tabular}{|c|c|c|c|c|c|c|}
\hline NYPMNNBWKKU & 73 & 490.2327 & 3 & 55.53 & 1467.688 & -8.2 \\
\hline DbMDAEjKMKU & 73 & 465.1997 & 3 & 55.73 & 1392.595 & -12.9 \\
\hline SYGAMSiFDKU & 73 & 619.7672 & 2 & 57.47 & 1237.531 & -8.6 \\
\hline DQDOUDTAQKU & 73 & 435.192 & 3 & 58.27 & 1302.545 & \\
\hline PXYAGDAYJKU & 73 & 685.3001 & 2 & 59.87 & 1368.592 & -4.3 \\
\hline PXYQADYZAKU & 73 & 685.3001 & 2 & 59.88 & 1368.596 & -7.5 \\
\hline AYQJCAFGAKU & 73 & 612.2767 & 2 & 66.88 & 1222.551 & -9.7 \\
\hline LZFDQEEIVKU & 73 & 498.2304 & 3 & 71.78 & 1491.685 & -10.1 \\
\hline WTbZNGWOFKU & 73 & 518.9019 & 3 & 72.49 & 1553.688 & -2.7 \\
\hline FQQJJTFEYKU & 73 & 541.2451 & 3 & 74.43 & 1620.723 & -6.1 \\
\hline jSFLSVMOGKU & 73 & 640.8087 & 2 & 81.31 & 1279.621 & -14.4 \\
\hline LAVDVZZMGKU & 73 & 611.3015 & 2 & 84.37 & 1220.597 & -7.1 \\
\hline YTSPNAWHjKU & 73 & 656.3096 & 2 & 84.52 & 1310.613 & -6.1 \\
\hline EZELPIDDSKU & 73 & 680.7932 & 2 & 86.11 & 1359.581 & -6.7 \\
\hline ZDYVLETjiKU & 73 & 752.8436 & 2 & 102.66 & 1503.683 & -6.9 \\
\hline TCbFQSTBTKU & 73 & 704.3112 & 2 & 118.97 & 1406.627 & -13.6 \\
\hline KHKYDGVBLKU & 72 & 341.1898 & 4 & 23.12 & 1360.742 & -8.6 \\
\hline KLKVACbGjKU & 72 & 424.9002 & 3 & 23.95 & 1271.696 & -13.3 \\
\hline CVGAVWNVEKU & 72 & 417.8849 & 3 & 27.46 & 1250.647 & -11.5 \\
\hline RjCCPLMTHKU & 72 & 432.8709 & 3 & 28.74 & 1295.602 & -8.6 \\
\hline GVSDVFRDMKU & 72 & 384.8663 & 3 & 29.65 & 1151.576 & 1. \\
\hline PNLKMQACMKU & 72 & 388.1951 & 3 & 31.34 & 1161.582 & -15.9 \\
\hline KYSFRAFSYKU & 72 & 324.6729 & 4 & 32.01 & 1294.682 & -15 \\
\hline KARDLSDNLKU & 72 & 386.8848 & 3 & 34.01 & 1157.652 & -16.5 \\
\hline MLVQGDPVGKU & 72 & 348.1889 & 3 & 34.04 & 1041.564 & -18.4 \\
\hline YIVLNCAPAKU & 72 & 453.9007 & 3 & 34.72 & 1358.691 & -8.2 \\
\hline jTHVNFEFYKU & 72 & 464.8886 & 3 & 36.4 & 1391.659 & -10.9 \\
\hline jTHVMRCFYKU & 72 & 464.8885 & 3 & 36.49 & 1391.656 & -9 \\
\hline MYOMTSTCGKU & 72 & 408.8388 & 3 & 37.95 & 1223.5 & -4.3 \\
\hline KVTLNMiZDKU & 72 & 459.5641 & 3 & 38.27 & 1375.679 & -5.9 \\
\hline LYMBWMGVGKU & 72 & 340.4185 & 4 & 38.84 & 1357.648 & -2 \\
\hline VDGVQVUbCKU & 72 & 415.5376 & 3 & 39.2 & 1243.595 & -3.1 \\
\hline cTAYNANVjKU & 72 & 428.2056 & 3 & 40.38 & 1281.615 & -15.8 \\
\hline TVPDMQFCAKU & 72 & 569.7644 & 2 & 41.03 & 1137.531 & -14.7 \\
\hline TMPDGMCTYKU & 72 & 573.2352 & 2 & 42.52 & 1144.471 & -13.6 \\
\hline DTADYTKYGKU & 72 & 580.7736 & 2 & 45.18 & 1159.551 & -15.9 \\
\hline DRWJETEQRKU & 72 & 378.9329 & 4 & 45.21 & 1511.719 & -10.7 \\
\hline WQYEABFMGKU & 72 & 478.5509 & 3 & 47.43 & 1432.64 & -6.4 \\
\hline MGMjEPJLKKU & 72 & 469.8905 & 3 & 50.08 & 1406.673 & -16.2 \\
\hline ALTDFHQEMKU & 72 & 406.8629 & 3 & 50.23 & 1217.586 & -15.9 \\
\hline SYLETEQDAKU & 72 & 591.7822 & 2 & 51.29 & 1181.556 & -5.5 \\
\hline PDWbSSTTAKU & 72 & 403.8566 & 3 & 53.19 & 1208.564 & -13.4 \\
\hline VcEQcGTEYKU & 72 & 670.8119 & 2 & 54.83 & 1339.62 & -8 \\
\hline SMLSZMMVNKU & 72 & 412.1926 & 3 & 55.44 & 1233.567 & -8.9 \\
\hline DKJYXDAJDKU & 72 & 535.891 & 3 & 55.89 & 1604.663 & -7 \\
\hline
\end{tabular}




\begin{tabular}{|c|c|c|c|c|c|c|}
\hline WGDRYCDGAKU & 72 & 585.2593 & 2 & 58.55 & 1168.508 & -3.6 \\
\hline NMEMSLSSSKU & 72 & 556.7492 & 2 & 60.48 & 1111.5 & -14.7 \\
\hline KDMJZGYMDKU & 72 & 483.1989 & 3 & 60.8 & 1446.598 & -15.8 \\
\hline VMEMSLSSSKU & 72 & 549.2618 & 2 & 63.43 & 1096.526 & -15.1 \\
\hline YWMAVMVXHKU & 72 & 461.8817 & 3 & 68.24 & 1382.649 & -18.3 \\
\hline EDKcWMAGFKU & 72 & 652.8066 & 2 & 68.51 & 1303.61 & -8.5 \\
\hline LMEMSLSSSKU & 72 & 556.2693 & 2 & 68.86 & 1110.541 & -15.4 \\
\hline DZFFPAPMRKU & 72 & 434.8784 & 3 & 71.87 & 1301.634 & -15.6 \\
\hline cCCHFVLLIKU & 72 & 482.9008 & 3 & 73.81 & 1445.688 & -5.2 \\
\hline TEWADVHcIKU & 72 & 490.5594 & 3 & 78.53 & 1468.667 & -7.1 \\
\hline EMWYQCFFSKU & 72 & 684.2886 & 2 & 79.64 & 1366.584 & -15.4 \\
\hline NENGYDVLOKU & 72 & 627.796 & 2 & 80.08 & 1253.59 & -10 \\
\hline NENTGEFLOKU & 72 & 627.7958 & 2 & 80.22 & 1253.59 & -10.3 \\
\hline UXYETFJADKU & 72 & 513.553 & 3 & 81.63 & 1537.64 & - \\
\hline LcSEDTMjBKU & 72 & 500.8893 & 3 & 81.89 & 1499.657 & -7.1 \\
\hline LAVDVFWNCKU & 72 & 597.3045 & 2 & 84.62 & 1192.606 & -9.8 \\
\hline FFAGYSRMYKU & 72 & 634.8077 & 2 & 85.42 & 1267.617 & -12.8 \\
\hline XHDLGWEXcKU & 72 & 506.5627 & 3 & 95.18 & 1516.654 & 8.3 \\
\hline XNMACMMTQKU & 72 & 712.7953 & 2 & 106.45 & 1423.597 & -14.9 \\
\hline NNVADRYCRKU & 71 & 413.2107 & 3 & 23.76 & 1236.615 & -3.5 \\
\hline GYVASQcUXKU & 71 & 449.2079 & 3 & 26.35 & 1344.615 & -9.7 \\
\hline TYQTSSTPRKU & 71 & 389.8677 & 3 & 26.88 & 1166.604 & -19.6 \\
\hline LPBYDDRQNKU & 71 & 474.8966 & 3 & 27.59 & 1421.685 & -12.2 \\
\hline KMPcMWTVEKU & 71 & 336.4189 & 4 & 28.47 & 1341.665 & -14 \\
\hline ZTVVHZMZOKU & 71 & 501.5587 & 3 & 29.67 & 1501.675 & -13.4 \\
\hline STADAKWEKKU & 71 & 388.209 & 3 & 30.09 & 1161.614 & -7.9 \\
\hline SDDEGAbTVKU & 71 & 569.7559 & 2 & 31.71 & 1137.512 & -12.8 \\
\hline WbSNRVGFGKU & 71 & 423.2171 & 3 & 32.27 & 1266.644 & -11.3 \\
\hline LBSAKCPZFKU & 71 & 341.4206 & 4 & 32.49 & 1361.672 & -13.4 \\
\hline TNFOYGQCjKU & 71 & 458.1998 & 3 & 32.51 & 1371.586 & -6.3 \\
\hline AMPALEGVLKU & 71 & 343.2034 & 3 & 32.58 & 1026.59 & -1.2 \\
\hline KGbPKDKDQKU & 71 & 420.8979 & 3 & 32.6 & 1259.68 & -6.5 \\
\hline VNEDDGVGTKU & 71 & 516.7442 & 2 & 35.38 & 1031.488 & -14 \\
\hline GKMNARQFLKU & 71 & 397.8924 & 3 & 37.06 & 1190.671 & -12.8 \\
\hline ZTVVXNEFDKU & 71 & 455.8745 & 3 & 37.6 & 1364.622 & -14.9 \\
\hline AFGAMGFTQKU & 71 & 528.7612 & 2 & 37.94 & 1055.522 & -13.7 \\
\hline KVTWDYKZDKU & 71 & 459.5641 & 3 & 38.38 & 1375.689 & -13.2 \\
\hline YAFPEQYDKKU & 71 & 429.8785 & 3 & 39.67 & 1286.629 & -12.1 \\
\hline DYTVPMPSGKU & 71 & 547.264 & 2 & 42.62 & 1092.527 & -12.8 \\
\hline NGSDEGIVVKU & 71 & 597.7858 & 2 & 44.9 & 1193.553 & 3.7 \\
\hline ALDMDSUIMKU & 71 & 662.2809 & 2 & 44.92 & 1322.561 & -10.6 \\
\hline DNESYDbSEKU & 71 & 652.2651 & 2 & 49.41 & 1302.518 & -1.8 \\
\hline KFMDWAVKQKU & 71 & 427.2307 & 3 & 49.6 & 1278.691 & -1 \\
\hline MSVVAGGNEKU & 71 & 495.7534 & 2 & 49.81 & 989.4964 & -4 \\
\hline KVGXKMRWLKU & 71 & 455.595 & 3 & 51.34 & 1363.777 & -10 \\
\hline
\end{tabular}




\begin{tabular}{r|rrrrrr} 
LNZWDPMVAKU & 71 & 423.2083 & 3 & 52.47 & 1266.618 & -11.8 \\
YSWTKGGNMKU & 71 & 390.8579 & 3 & 52.74 & 1169.565 & -11.4 \\
QQbjQSHJQKU & 71 & 525.9037 & 3 & 52.89 & 1574.708 & -11.9 \\
YDYFYPKELKU & 71 & 455.5613 & 3 & 54.64 & 1363.681 & -13.9 \\
TVSMYSVMNKU & 71 & 579.7793 & 2 & 54.77 & 1157.557 & -11.5 \\
YNLDPMZJKKU & 71 & 490.233 & 3 & 55.32 & 1467.689 & -7.8 \\
VKWQEGQcEKU & 71 & 442.2246 & 3 & 59.88 & 1323.665 & -9.9 \\
EMEMSLSSSKU & 71 & 564.2472 & 2 & 61.72 & 1126.5 & -17.7 \\
UPHLFARTEKU & 71 & 426.5624 & 3 & 63.46 & 1276.679 & -10.4 \\
ACTKTSjSYKU & 71 & 598.7842 & 2 & 67 & 1195.563 & -7.2 \\
CAGVQWWFWKU & 71 & 655.313 & 2 & 67.12 & 1308.623 & -8.4 \\
KYUGCCAIYKU & 71 & 469.1968 & 3 & 67.76 & 1404.592 & -16.5 \\
EDKSFGXQFKU & 71 & 652.8059 & 2 & 68.79 & 1303.606 & -6.5 \\
KNCLSDOEVKU & 71 & 462.8957 & 3 & 69.37 & 1385.687 & -15.3 \\
PUPDZSDMVKU & 71 & 631.778 & 2 & 71.28 & 1261.551 & -7.6 \\
XDEGFVLIKU & 71 & 477.5696 & 3 & 73.73 & 1429.695 & -5.7 \\
\hline GANWUBQBFKU & 71 & 790.353 & 2 & 80.8 & 1578.708 & -10.6 \\
FMAWLYGNEKU & 71 & 629.2978 & 2 & 91.2 & 1256.601 & -16 \\
ASCCWWVCIKU & 71 & 489.5521 & 3 & 95.2 & 1465.62 & 9.6 \\
XSDFQSTBTKU & 71 & 704.3111 & 2 & 119.52 & 1406.613 & -3.7
\end{tabular}


Library QC sample 2

$U=C$-terminal amide, $B=D a b A, C=D a b C, I=D a b G, J=D a b T, O=A l a A, U=A l a C, X=A l a G, Z=A l a T$, $b=h A l a A, c=h A l a C, i=h A l a G, j=h A l a T$

\begin{tabular}{|c|c|c|c|c|c|c|}
\hline Peptide & $A L C(\%)$ & $m / z$ & $z$ & $R T$ & Mass & ppm \\
\hline LNbVPMTDJKU & 99 & 467.5568 & 3 & 41.42 & 1399.669 & -14.5 \\
\hline OGFDLSHTEKU & 99 & 412.8596 & 3 & 55.79 & 1235.58 & -18.3 \\
\hline PPMESMSAMKU & 98 & 369.8318 & 3 & 27.29 & 1106.492 & -16.9 \\
\hline ZDERQUSQMKU & 98 & 465.8722 & 3 & 32.63 & 1394.611 & -11.5 \\
\hline VGVPVLWGEKU & 98 & 361.5452 & 3 & 37.22 & 1081.628 & -13.5 \\
\hline jPQRCYEMMKU & 98 & 465.2047 & 3 & 43.03 & 1392.607 & -10.7 \\
\hline QELQDYYKGKU & 98 & 424.2114 & 3 & 43.8 & 1269.635 & -18.1 \\
\hline PYFJEYSYLKU & 98 & 492.2309 & 3 & 48.59 & 1473.689 & -12 \\
\hline AYPNFLMPHKU & 98 & 406.2074 & 3 & 50.85 & 1215.622 & -18.1 \\
\hline AYPNFPMVHKU & 98 & 401.5353 & 3 & 52.22 & 1201.607 & -18.9 \\
\hline DSATEVGWMKU & 97 & 561.759 & 2 & 24.6 & 1121.518 & -12.5 \\
\hline LVGVNRONYKU & 97 & 317.1764 & 4 & 25.01 & 1264.69 & -10.8 \\
\hline APVEAGWAFKU & 97 & 358.8577 & 3 & 27.47 & 1073.566 & -13.4 \\
\hline ASNLPEECJKU & 97 & 628.2813 & 2 & 28.85 & 1254.562 & -10.9 \\
\hline FCGSQWVEKKU & 97 & 404.1996 & 3 & 28.92 & 1209.596 & -16.1 \\
\hline GVNTVYDYYKU & 97 & 407.5325 & 3 & 29.6 & 1219.587 & -9.7 \\
\hline DHFNQQFDJKU & 97 & 481.882 & 3 & 30.83 & 1442.628 & -3 \\
\hline DXVQYQKWAKU & 97 & 462.232 & 3 & 33.27 & 1383.679 & -3.8 \\
\hline FHGVAYVFXKU & 97 & 429.5515 & 3 & 38.6 & 1285.647 & -11 \\
\hline FLNAGQSSSKU & 97 & 519.2648 & 2 & 44.01 & 1036.53 & -14.5 \\
\hline WNKLDTFPMKU & 97 & 426.886 & 3 & 51.11 & 1277.659 & -17.9 \\
\hline YDLSYNjSBKU & 97 & 491.5511 & 3 & 51.93 & 1471.65 & -12.9 \\
\hline DAFFNPDPcKU & 97 & 415.2008 & 3 & 56.74 & 1242.575 & 4.5 \\
\hline TjOGGYLQVKU & 97 & 639.3158 & 2 & 61.41 & 1276.639 & -17.5 \\
\hline PVVGELDVQKU & 97 & 541.8057 & 2 & 61.96 & 1081.613 & -15 \\
\hline ZEYDTSFBFKU & 97 & 502.5467 & 3 & 63.75 & 1504.642 & -16 \\
\hline LYYRLSMPYKU & 97 & 444.9071 & 3 & 64.64 & 1331.706 & -4.9 \\
\hline KYAPFFDAYKU & 97 & 416.8812 & 3 & 83.71 & 1247.634 & -9.6 \\
\hline TKGSQQLDYKU & 96 & 389.537 & 3 & 29.44 & 1165.609 & -17.2 \\
\hline GVEMVPMEcKU & 96 & 404.8606 & 3 & 35.57 & 1211.576 & -13.2 \\
\hline GVEETVEEcKU & 96 & 404.8596 & 3 & 35.67 & 1211.575 & -14.7 \\
\hline QMEFYADLbKU & 96 & 454.5433 & 3 & 36.98 & 1360.63 & -16.1 \\
\hline KSHTYDQLSKU & 96 & 402.5405 & 3 & 41.64 & 1204.62 & -17.1 \\
\hline VNFDSKGEVKU & 96 & 374.5294 & 3 & 46.93 & 1120.588 & -18.9 \\
\hline LEEjVKPADKU & 96 & 412.8874 & 3 & 55.12 & 1235.648 & -6.2 \\
\hline INFYSZEFYKU & 96 & 528.2289 & 3 & 61.94 & 1581.674 & -5.9 \\
\hline EPPPCLVFYKU & 96 & 596.3054 & 2 & 64.12 & 1190.616 & -16.4 \\
\hline DSMSEFLYDKU & 96 & 617.2769 & 2 & 86.66 & 1232.538 & 0.7 \\
\hline GLLJQDTYJKU & 95 & 490.2335 & 3 & 22.63 & 1467.702 & -15.8 \\
\hline
\end{tabular}




\begin{tabular}{|c|c|c|c|c|c|c|}
\hline VSTYBSWGYKU & 95 & 455.5468 & 3 & 23.63 & 1363.636 & -12.9 \\
\hline ADUEMQFDNKU & 95 & 426.1833 & 3 & 24.45 & 1275.53 & -1.6 \\
\hline FVDTGAWAJKU & 95 & 420.5369 & 3 & 24.48 & 1258.605 & -12.9 \\
\hline VUKNDSDcHKU & 95 & 439.2052 & 3 & 27.21 & 1314.615 & -15.8 \\
\hline APMPAGEKFKU & 95 & 358.8579 & 3 & 27.74 & 1073.569 & -15.9 \\
\hline YSGSQWNNKKU & 95 & 404.1997 & 3 & 28.89 & 1209.589 & -9.8 \\
\hline POMPNANVjKU & 95 & 428.2049 & 3 & 39.48 & 1281.612 & -14.9 \\
\hline jNSSMGDFLKU & 95 & 603.7717 & 2 & 43.84 & 1205.547 & -14.9 \\
\hline VFAPLERATKU & 95 & 377.5577 & 3 & 43.93 & 1129.661 & -8.4 \\
\hline EGLAEOELIKU & 95 & 442.5533 & 3 & 44.32 & 1324.639 & -0.7 \\
\hline jSDCEGTYiKU & 95 & 498.2087 & 3 & 49.15 & 1491.617 & -8.5 \\
\hline VOMPYGPKKKU & 95 & 417.5677 & 3 & 50.2 & 1249.687 & -4.2 \\
\hline ELLGDTYCMKU & 95 & 586.2695 & 2 & 52.42 & 1170.541 & -14.3 \\
\hline DAFFNPDYKKU & 95 & 415.2003 & 3 & 57.09 & 1242.603 & -19.4 \\
\hline YDLVDCPPVKU & 95 & 574.2867 & 2 & 59.18 & 1146.574 & -13.3 \\
\hline LYVXLAFPYKU & 95 & 444.907 & 3 & 64.53 & 1331.714 & -11 \\
\hline OGCDCLPQQKU & 94 & 497.5651 & 3 & 25.27 & 1489.694 & -13.9 \\
\hline EYVSRAVSQKU & 94 & 389.212 & 3 & 29.13 & 1164.625 & -9.2 \\
\hline FTXSDETNSKU & 94 & 416.5114 & 3 & 29.32 & 1246.533 & -16.3 \\
\hline VFAPLQTRAKU & 94 & 377.2294 & 3 & 31.15 & 1128.677 & -9.2 \\
\hline ACFFYEHRVKU & 94 & 433.5463 & 3 & 31.93 & 1297.639 & -16.8 \\
\hline VDTCYCFNbKU & 94 & 486.5453 & 3 & 32.35 & 1456.633 & -12.7 \\
\hline FKQUTKPLAKU & 94 & 413.9104 & 3 & 33.29 & 1238.725 & -12.4 \\
\hline FKLZTQPLAKU & 94 & 413.9118 & 3 & 33.35 & 1238.714 & \\
\hline GVVDFiAALKU & 94 & 384.881 & 3 & 36.1 & 1151.621 & 0.6 \\
\hline LQVEMHCPMKU & 94 & 405.5264 & 3 & 37.69 & 1213.577 & -16.1 \\
\hline VFCLYCETSKU & 94 & 428.2084 & 3 & 38.48 & 1281.614 & -8.6 \\
\hline IQNMKLEQSKU & 94 & 465.8959 & 3 & 39.22 & 1394.683 & -12.2 \\
\hline XHZAMELPCKU & 94 & 478.5568 & 3 & 39.93 & 1432.65 & -0.8 \\
\hline VZBYRFLYQKU & 94 & 529.2721 & 3 & 40.03 & 1584.8 & -3.7 \\
\hline AFMASDSETKU & 94 & 543.2437 & 2 & 41.17 & 1084.486 & -12.1 \\
\hline QKMSCMFQMKU & 94 & 420.8582 & 3 & 43.94 & 1259.565 & -9.4 \\
\hline TGXLDILPQKU & 94 & 461.2252 & 3 & 44.76 & 1380.675 & -15.5 \\
\hline QPELGVTTjKU & 94 & 394.2074 & 3 & 45.07 & 1179.622 & -18.1 \\
\hline QELEDYYKGKU & 94 & 424.5395 & 3 & 46.62 & 1270.619 & -17.8 \\
\hline GFAVHNNJMKU & 94 & 428.2041 & 3 & 48.46 & 1281.599 & -6.9 \\
\hline QTYDEAELTKU & 94 & 598.7834 & 2 & 51.04 & 1195.572 & -16.6 \\
\hline jDbLPLRCYKU & 94 & 478.5716 & 3 & 51.33 & 1432.707 & -10.1 \\
\hline FYGVAYVOYKU & 94 & 438.2183 & 3 & 54.75 & 1311.651 & -13.7 \\
\hline LMIYCYFFDKU & 94 & 760.3266 & 2 & 67.14 & 1518.653 & -9.3 \\
\hline VYRAFYCGSKU & 93 & 398.1991 & 3 & 27.2 & 1191.586 & -8.7 \\
\hline PAVEAGAWFKU & 93 & 358.8577 & 3 & 27.48 & 1073.566 & -13.4 \\
\hline VQUBMPVPQKU & 93 & 460.8977 & 3 & 27.5 & 1379.693 & -16.1 \\
\hline DHFGGKBDJKU & 93 & 481.882 & 3 & 28.3 & 1442.645 & -14.2 \\
\hline GVVAQQCVLKU & 93 & 397.8981 & 3 & 34.12 & 1190.684 & -9 \\
\hline
\end{tabular}




\begin{tabular}{|c|c|c|c|c|c|c|}
\hline CPPMPMGVYKU & 93 & 374.5125 & 3 & 36.41 & 1120.523 & -6.6 \\
\hline LVGEFVBWKKU & 93 & 345.6898 & 4 & 37.46 & 1378.756 & -19.2 \\
\hline QLVEDHSEMKU & 93 & 405.5264 & 3 & 37.62 & 1213.576 & -15.4 \\
\hline IQNCRLEQSKU & 93 & 465.8877 & 3 & 39.3 & 1394.658 & -11.7 \\
\hline NMOPANILLKU & 93 & 446.5581 & 3 & 40.95 & 1336.669 & -12.4 \\
\hline FMLCFNjSBKU & 93 & 491.5519 & 3 & 42.63 & 1471.651 & -11.9 \\
\hline QNDJMELYKKU & 93 & 478.5569 & 3 & 43.67 & 1432.673 & -16.5 \\
\hline GVjNDFOGLKU & 93 & 421.2052 & 3 & 45.08 & 1260.608 & -11.3 \\
\hline NNVCXGEPAKU & 93 & 621.2866 & 2 & 52.43 & 1240.578 & -15.3 \\
\hline LVFPGVIOFKU & 93 & 467.5802 & 3 & 53.59 & 1399.737 & -12.7 \\
\hline MMRMMTVGEKU & 93 & 404.8588 & 3 & 54.1 & 1211.565 & -8.3 \\
\hline SEMNEVZMLKU & 93 & 637.7897 & 2 & 55.55 & 1273.58 & -11.5 \\
\hline TjGFXLAGVKU & 93 & 610.8073 & 2 & 59.31 & 1219.618 & -14.6 \\
\hline LYYLAVOPYKU & 93 & 666.8578 & 2 & 64.57 & 1331.714 & -9.6 \\
\hline LMIYJGUIDKU & 93 & 585.2448 & 3 & 66.79 & 1752.734 & -12.1 \\
\hline MGFTCSLLFKU & 93 & 382.5272 & 3 & 78.33 & 1144.577 & -15.4 \\
\hline YUUYGCYFKKU & 92 & 477.5469 & 3 & 24.92 & 1429.635 & -11.2 \\
\hline KLTPFCbCSKU & 92 & 415.2052 & 3 & 26.52 & 1242.607 & -10.6 \\
\hline YQTCLLGVAKU & 92 & 365.5367 & 3 & 26.53 & 1093.596 & -6.5 \\
\hline jFjXGGKPSKU & 92 & 453.2116 & 3 & 31.79 & 1356.638 & -18.3 \\
\hline LGNCNLGLKKU & 92 & 353.5387 & 3 & 37.17 & 1057.607 & -11.8 \\
\hline DWDLNANVjKU & 92 & 428.2051 & 3 & 39.38 & 1281.607 & -10.7 \\
\hline SAXYYPTUAKU & 92 & 433.8692 & 3 & 40.93 & 1298.59 & -3.6 \\
\hline AFMACAMETKU & 92 & 551.2404 & 2 & 41.31 & 1100.482 & -14.1 \\
\hline NDCFKPMMYKU & 92 & 456.209 & 3 & 42.71 & 1365.629 & -17.3 \\
\hline FDBINDAYHKU & 92 & 394.4185 & 4 & 43.27 & 1573.66 & -9.8 \\
\hline VDVGPETFFKU & 92 & 379.8652 & 3 & 45.76 & 1136.587 & -11.3 \\
\hline UNSOEMWYFKU & 92 & 496.5421 & 3 & 47.63 & 1486.631 & -17.8 \\
\hline VEYFEbMYDKU & 92 & 480.8782 & 3 & 47.82 & 1439.625 & -8.4 \\
\hline jYVGGYLjiKU & 92 & 725.8374 & 2 & 63.71 & 1449.685 & -16.9 \\
\hline FTFQSMQMMKU & 92 & 639.2847 & 2 & 67.93 & 1276.577 & -17 \\
\hline IQNMQLEQSKU & 91 & 465.8871 & 3 & 23.52 & 1394.647 & -5.1 \\
\hline SLAMTCPZTKU & 91 & 573.2679 & 2 & 23.56 & 1144.537 & -13.6 \\
\hline EYTZNjRIGKU & 91 & 521.2375 & 3 & 24.76 & 1560.689 & 0.9 \\
\hline KLNCKEUDSKU & 91 & 415.2057 & 3 & 26.43 & 1242.614 & -14.8 \\
\hline bAGVSKSJLKU & 91 & 405.89 & 3 & 27.51 & 1214.656 & -6.4 \\
\hline LNPGEPGJNKU & 91 & 397.5318 & 3 & 28.93 & 1189.58 & -5.1 \\
\hline NIDPYHRRFKU & 91 & 508.2516 & 3 & 31.32 & 1521.744 & -7.2 \\
\hline RUVPGCTFFKU & 91 & 309.1585 & 4 & 32.66 & 1232.624 & -15.1 \\
\hline FKLPUFPLAKU & 91 & 310.6852 & 4 & 33.25 & 1238.729 & -13.6 \\
\hline FUFIAPEGVKU & 91 & 341.9186 & 4 & 34.54 & 1363.653 & -5.3 \\
\hline MMNKQFDYCKU & 91 & 436.1967 & 3 & 36.97 & 1305.567 & 1 \\
\hline EEAPPJOADKU & 91 & 442.5327 & 3 & 39.83 & 1324.586 & -7.6 \\
\hline KYLYEMUMYKU & 91 & 483.2296 & 3 & 45.62 & 1446.679 & -8.1 \\
\hline CHAEQcULcKU & 91 & 515.2433 & 3 & 48.81 & 1542.719 & -6.8 \\
\hline
\end{tabular}




\begin{tabular}{|c|c|c|c|c|c|c|}
\hline EGQDNOPbMKU & 91 & 447.195 & 3 & 49.36 & 1338.57 & -5.4 \\
\hline KYMPLELOYKU & 91 & 463.2454 & 3 & 49.89 & 1386.723 & -6.1 \\
\hline MPYYMMWOMKU & 91 & 495.209 & 3 & 50.5 & 1482.618 & -8.7 \\
\hline MYLCFNjSBKU & 91 & 496.8815 & 3 & 52.09 & 1487.646 & -15.9 \\
\hline VVSPYTPbAKU & 91 & 393.5453 & 3 & 59.78 & 1177.631 & -14.3 \\
\hline XANJMZEFYKU & 91 & 528.2172 & 3 & 61.01 & 1581.649 & -11.9 \\
\hline OSNMJZEFYKU & 91 & 528.2169 & 3 & 61.13 & 1581.649 & -12.6 \\
\hline INFYYCEYYKU & 91 & 528.2153 & 3 & 61.36 & 1581.645 & -13.2 \\
\hline TjGFGWFQVKU & 91 & 639.3158 & 2 & 61.42 & 1276.632 & -12 \\
\hline cYZAMQNYKKU & 91 & 717.3338 & 2 & 71.17 & 1432.664 & -7.5 \\
\hline DPTUMSSCLKU & 90 & 436.8684 & 3 & 23.01 & 1307.599 & -12.2 \\
\hline ADSMYAWJDKU & 90 & 451.1884 & 3 & 24.19 & 1350.562 & -13.7 \\
\hline DSEASKDSjKU & 90 & 392.1765 & 3 & 24.3 & 1173.523 & -13.1 \\
\hline ZKMGTFKLGKU & 90 & 401.8868 & 3 & 24.81 & 1202.659 & -17.2 \\
\hline EMEMASCPMKU & 90 & 416.1761 & 3 & 28.04 & 1245.527 & -16.5 \\
\hline KYXPECLVGKU & 90 & 468.5761 & 3 & 30.84 & 1402.717 & -7.6 \\
\hline LQNQUZACKU & 90 & 689.3266 & 2 & 33.32 & 1376.658 & -13.7 \\
\hline MOVQYQKWAKU & 90 & 462.2332 & 3 & 33.5 & 1383.698 & -14.9 \\
\hline VMMRGLXSXKU & 90 & 454.2179 & 3 & 33.82 & 1359.651 & -14.2 \\
\hline VGXBDNYLVKU & 90 & 467.8948 & 3 & 35.47 & 1400.675 & -8.8 \\
\hline LZGMjTEYAKU & 90 & 658.2991 & 2 & 35.91 & 1314.6 & -12.3 \\
\hline GIIFMQPLZKU & 90 & 532.913 & 3 & 39.06 & 1595.726 & -5.5 \\
\hline SAXUDKTUAKU & 90 & 433.8693 & 3 & 40.76 & 1298.598 & -9 \\
\hline QNGGEDQDLKU & 90 & 551.7497 & 2 & 41.45 & 1101.505 & -18.4 \\
\hline LQWVFOSJAKU & 90 & 464.2353 & 3 & 44.12 & 1389.702 & -13.1 \\
\hline LVDKGHSbRKU & 90 & 419.5656 & 3 & 45.96 & 1255.697 & -17.1 \\
\hline bLGTCLAVHKU & 90 & 386.8734 & 3 & 49.4 & 1157.62 & -18.4 \\
\hline DYLSYNjBSKU & 90 & 491.5524 & 3 & 51.79 & 1471.65 & -10.1 \\
\hline SAFLJGOGGKU & 90 & 603.288 & 2 & 52.84 & 1204.581 & -15.8 \\
\hline TSGFXLAGVKU & 90 & 549.7845 & 2 & 54.31 & 1097.573 & -16.8 \\
\hline QNNKTYDjSKU & 90 & 435.873 & 3 & 57.32 & 1304.608 & -8.3 \\
\hline ELLGDTCFMKU & 90 & 578.2717 & 2 & 78.04 & 1154.546 & -15.1 \\
\hline VVPBVDAFFKU & 90 & 432.5632 & 3 & 88.01 & 1294.688 & -15.2 \\
\hline FKQTUKPLAKU & 89 & 310.6847 & 4 & 26.67 & 1238.725 & -12 \\
\hline LSCSTUAFIKU & 89 & 442.867 & 3 & 27.3 & 1325.604 & -18.5 \\
\hline SLAMTCPjSKU & 89 & 382.5132 & 3 & 27.75 & 1144.534 & -14.2 \\
\hline LPPGYLPMGKU & 89 & 357.8697 & 3 & 27.99 & 1070.595 & -7.1 \\
\hline MAAGSTFVXKU & 89 & 377.5187 & 3 & 28.28 & 1129.545 & -9.4 \\
\hline DHFNKGbDJKU & 89 & 481.8811 & 3 & 28.55 & 1442.646 & -17.1 \\
\hline GVYDLOPKKKU & 89 & 417.5693 & 3 & 30.12 & 1249.704 & -14.7 \\
\hline VODLYGPKKKU & 89 & 417.5691 & 3 & 30.21 & 1249.704 & -15.1 \\
\hline EFGQGSVMPKU & 89 & 539.7638 & 2 & 34.68 & 1077.528 & -13.5 \\
\hline TYKDDDCMEKU & 89 & 465.5366 & 3 & 35.17 & 1393.588 & -0.4 \\
\hline ZMGKAHFANKU & 89 & 599.2935 & 2 & 36.46 & 1196.587 & -12.6 \\
\hline VABYRYPYKKU & 89 & 366.1998 & 4 & 36.92 & 1460.773 & -1.9 \\
\hline
\end{tabular}




\begin{tabular}{|c|c|c|c|c|c|c|}
\hline AVHFSEAAJKU & 89 & 408.8685 & 3 & 38.75 & 1223.6 & -13.6 \\
\hline ESMNEVZMLKU & 89 & 637.79 & 2 & 39.25 & 1273.58 & -11.1 \\
\hline cYZCDELYKKU & 89 & 483.8885 & 3 & 39.84 & 1448.648 & -2.5 \\
\hline YLOYCRjEDKU & 89 & 501.2234 & 3 & 40.26 & 1500.665 & -11 \\
\hline cLGNUQMQVKU & 89 & 430.883 & 3 & 40.35 & 1289.638 & -8.4 \\
\hline AYPSSSLVHKU & 89 & 363.1943 & 3 & 45.42 & 1086.582 & -19.6 \\
\hline USDUGDMTVKU & 89 & 404.5049 & 3 & 46.7 & 1210.515 & -18.2 \\
\hline LQFAGBjSHKU & 89 & 457.5558 & 3 & 47.85 & 1369.666 & -15.1 \\
\hline KGYMQSVMXKU & 89 & 430.8722 & 3 & 54.38 & 1289.612 & -13.5 \\
\hline jDDDEXFFFKU & 89 & 497.5324 & 3 & 58.04 & 1489.598 & -15.3 \\
\hline KYLQDUUMYKU & 89 & 483.2303 & 3 & 59.41 & 1446.683 & -9.3 \\
\hline INFFCZEFYKU & 89 & 528.2289 & 3 & 61.95 & 1581.656 & 5.4 \\
\hline GEYFFMZYKKU & 89 & 469.5519 & 3 & 66.38 & 1405.649 & -10.8 \\
\hline SDMSEFLMFKU & 89 & 617.2755 & 2 & 86.76 & 1232.557 & -16.6 \\
\hline LVjcSPMSNKU & 88 & 426.5443 & 3 & 23.36 & 1276.628 & -13.6 \\
\hline EGAERWKYEKU & 88 & 432.2209 & 3 & 24.19 & 1293.647 & -4.2 \\
\hline MDVGSUCPVKU & 88 & 557.7529 & 2 & 25.15 & 1113.506 & -13.1 \\
\hline GOCDILNQQKU & 88 & 497.5651 & 3 & 25.28 & 1489.674 & -0.5 \\
\hline LPPGYGRQGKU & 88 & 357.8711 & 3 & 25.93 & 1070.598 & -6.6 \\
\hline GVLOCMјAHKU & 88 & 473.5644 & 3 & 26.72 & 1417.682 & -7.6 \\
\hline FMAHCNCTQKU & 88 & 443.8612 & 3 & 28.52 & 1328.582 & -15.2 \\
\hline TPKYMGEBHKU & 88 & 455.5491 & 3 & 28.66 & 1363.651 & -18.6 \\
\hline FCGSQWPMKKU & 88 & 404.1996 & 3 & 28.92 & 1209.579 & -1.4 \\
\hline ATMUNKEjbKU & 88 & 476.5513 & 3 & 29.79 & 1426.656 & -16.9 \\
\hline KYMGQNCLRKU & 88 & 463.2448 & 3 & 30.81 & 1386.726 & -9.4 \\
\hline FPTCYRQXJKU & 88 & 509.9017 & 3 & 31.73 & 1526.69 & -4.7 \\
\hline jFjYELGPSKU & 88 & 453.2128 & 3 & 32.64 & 1356.64 & -17.5 \\
\hline LVDWAPSWRKU & 88 & 419.5656 & 3 & 34.6 & 1255.683 & -6.2 \\
\hline LEFMKMCOQKU & 88 & 454.2178 & 3 & 34.81 & 1359.636 & -3.3 \\
\hline ZcMSQPNMYKU & 88 & 462.8687 & 3 & 36.86 & 1385.594 & -6.9 \\
\hline PcEYNPPFYKU & 88 & 449.8809 & 3 & 37.71 & 1346.638 & -12.3 \\
\hline DSEASRCAjKU & 88 & 587.7615 & 2 & 38.82 & 1173.517 & -7 \\
\hline ATMUEELjbKU & 88 & 476.5501 & 3 & 39.64 & 1426.645 & -11.6 \\
\hline LENKQFDCYKU & 88 & 429.5464 & 3 & 42.63 & 1285.613 & 3.9 \\
\hline WNZSGTESDKU & 88 & 609.253 & 2 & 45.13 & 1216.511 & -16.1 \\
\hline jNSSSTELDKU & 88 & 594.7668 & 2 & 46.14 & 1187.539 & -16.6 \\
\hline EYGHMMMjAKU & 88 & 435.8466 & 3 & 46.2 & 1304.544 & -19.5 \\
\hline LNVLDTDCYKU & 88 & 591.7916 & 2 & 47.41 & 1181.575 & -5.3 \\
\hline ELLGDTFCMKU & 88 & 578.2724 & 2 & 52.39 & 1154.546 & -14 \\
\hline VEYFEjjLYKU & 88 & 503.236 & 3 & 57.25 & 1506.709 & -14.8 \\
\hline EGAMVFAQSKU & 88 & 533.7623 & 2 & 59 & 1065.528 & -16.4 \\
\hline YDLVDEGNPKU & 88 & 574.7765 & 2 & 60.48 & 1147.551 & -11 \\
\hline ENEVGYLjiKU & 88 & 697.3271 & 2 & 61.59 & 1392.651 & -8.2 \\
\hline FPVVQXGTQKU & 88 & 611.8168 & 2 & 64.39 & 1221.637 & -14.4 \\
\hline ЕРPЕАКСКYKU & 88 & 596.3071 & 2 & 64.41 & 1190.612 & -10 . \\
\hline
\end{tabular}




\begin{tabular}{|c|c|c|c|c|c|c|}
\hline VQSNVPFCcKU & 87 & 405.534 & 3 & 24.52 & 1213.599 & -15.9 \\
\hline MSVVCPHVEKU & 87 & 376.5225 & 3 & 28.99 & 1126.563 & -15.2 \\
\hline WLDYMSDRKKU & 87 & 447.5555 & 3 & 29.54 & 1339.671 & -19.4 \\
\hline VOLYDGPKKKU & 87 & 417.5693 & 3 & 30.12 & 1249.704 & -14.7 \\
\hline LZTSCTbZUKU & 87 & 480.5421 & 3 & 30.57 & 1438.623 & -12.8 \\
\hline MYVSYPGXRKU & 87 & 440.5474 & 3 & 30.72 & 1318.635 & -11.4 \\
\hline GHXGNQVMXKU & 87 & 437.1948 & 3 & 31.28 & 1308.575 & -9.9 \\
\hline DANPYGjiZKU & 87 & 467.868 & 3 & 35.97 & 1400.595 & -8.9 \\
\hline GcHFYAMPbKU & 87 & 454.5433 & 3 & 36.98 & 1360.628 & -14.7 \\
\hline VABYRFLPCKU & 87 & 366.1991 & 4 & 37 & 1460.781 & -9.3 \\
\hline EGGFDEIWAKU & 87 & 424.5216 & 3 & 38.75 & 1270.549 & -4.3 \\
\hline QDLQTFMjSKU & 87 & 435.8716 & 3 & 41.52 & 1304.616 & -17.3 \\
\hline EQVEAYjKFKU & 87 & 450.5638 & 3 & 41.54 & 1348.675 & -3.6 \\
\hline FUCESMMBQKU & 87 & 535.8911 & 3 & 43.56 & 1604.673 & -13.5 \\
\hline GVNTVYMFIKU & 87 & 450.2173 & 3 & 46.37 & 1347.65 & -14.6 \\
\hline PNYFJQXCLKU & 87 & 794.8656 & 2 & 51.71 & 1587.737 & -12.5 \\
\hline KXMQSVMYGKU & 87 & 430.8709 & 3 & 54.24 & 1289.612 & -16.5 \\
\hline ZAPAYPVMXKU & 87 & 645.804 & 2 & 54.25 & 1289.609 & -11.8 \\
\hline CbLYPVZDGKU & 87 & 436.2035 & 3 & 54.4 & 1305.599 & -8.1 \\
\hline CHLQYDYKGKU & 87 & 627.3029 & 2 & 60.69 & 1252.602 & -8.8 \\
\hline CHCPUjLLCKU & 86 & 386.6838 & 4 & 24.28 & 1542.727 & -13.2 \\
\hline GVCSSEMRKKU & 86 & 375.1893 & 3 & 26.08 & 1122.564 & -15.7 \\
\hline WQQQQMAQZKU & 86 & 457.2176 & 3 & 26.32 & 1368.636 & -3.5 \\
\hline DAHXDFRLGKU & 86 & 426.5452 & 3 & 27.03 & 1276.617 & -2.7 \\
\hline GTBZbCVMTKU & 86 & 476.2077 & 3 & 28.18 & 1425.615 & -9.6 \\
\hline FTXDCETNSKU & 86 & 421.8428 & 3 & 29.38 & 1262.51 & -2.6 \\
\hline IFGLECLGVKU & 86 & 351.6835 & 4 & 30.75 & 1402.716 & -7.9 \\
\hline KYLYCjYRCKU & 86 & 483.2292 & 3 & 31.29 & 1446.687 & -14.6 \\
\hline DHFELQEDJKU & 86 & 475.8783 & 3 & 31.31 & 1424.628 & -10.2 \\
\hline GEVPYNGDAKU & 86 & 350.1753 & 3 & 31.64 & 1047.499 & 5.3 \\
\hline ZPVECGPKFKU & 86 & 400.1996 & 3 & 34.43 & 1197.596 & -16.2 \\
\hline LQNQUCGjKU & 86 & 689.3276 & 2 & 36.12 & 1376.655 & -10.1 \\
\hline ZMGQANQLNKU & 86 & 599.2942 & 2 & 36.52 & 1196.572 & 1.5 \\
\hline TYLVTETNNKU & 86 & 394.5377 & 3 & 37.57 & 1180.609 & -15 \\
\hline LMPNVGBLKU & 86 & 471.2402 & 3 & 38.34 & 1410.72 & -15 \\
\hline WAVEAYjKFKU & 86 & 338.1744 & 4 & 41.54 & 1348.69 & -16 \\
\hline AYVSGDLVHKU & 86 & 363.1941 & 3 & 45.12 & 1086.582 & -20 \\
\hline SDUSLWNDXKU & 86 & 455.1969 & 3 & 45.89 & 1362.581 & -9.1 \\
\hline VEYTTCUSPKU & 86 & 603.7745 & 2 & 47.94 & 1205.55 & -12.7 \\
\hline FMLSYTMCDKU & 86 & 462.5402 & 3 & 51.05 & 1384.622 & -16.8 \\
\hline MSPNYLDVHKU & 86 & 401.5354 & 3 & 52.3 & 1201.591 & -5.8 \\
\hline QNLGDTYMCKU & 86 & 586.2681 & 2 & 52.55 & 1170.516 & 4.8 \\
\hline jDLPLSATYKU & 86 & 608.3137 & 2 & 53.32 & 1214.627 & -11.3 \\
\hline VGAVIEALFKU & 86 & 583.8209 & 2 & 56.51 & 1165.636 & -7.7 \\
\hline TEFYMNLPQKU & 86 & 635.3097 & 2 & 68.97 & 1268.622 & -13.8 \\
\hline
\end{tabular}




\begin{tabular}{|c|c|c|c|c|c|c|}
\hline YYLAFVbMCKU & 86 & 452.215 & 3 & 71.66 & 1353.643 & -14.8 \\
\hline OSDQPFFIWKU & 86 & 516.8992 & 3 & 73.94 & 1547.691 & -9.7 \\
\hline VRXGGKLSTKU & 85 & 388.8875 & 3 & 21.44 & 1163.664 & -19.7 \\
\hline ADUECRFDNKU & 85 & 319.8891 & 4 & 24.46 & 1275.542 & -11 \\
\hline DHFELQMMJKU & 85 & 481.882 & 3 & 28.3 & 1442.639 & -10.3 \\
\hline BDjDLYLGVKU & 85 & 469.2306 & 3 & 29.02 & 1404.681 & -7.9 \\
\hline QRMFYLTCHKU & 85 & 332.165 & 4 & 29.18 & 1324.653 & -16.8 \\
\hline VOEMFGPKKKU & 85 & 422.9005 & 3 & 30.47 & 1265.681 & -1.5 \\
\hline DNTFKGANDKU & 85 & 370.1813 & 3 & 35.14 & 1107.531 & -7.9 \\
\hline DAGGPYJiZKU & 85 & 467.8682 & 3 & 35.83 & 1400.593 & -7.5 \\
\hline GGXXEDGZFKU & 85 & 448.5117 & 3 & 44.54 & 1342.53 & -12.3 \\
\hline AGPQNRTYFKU & 85 & 394.2075 & 3 & 44.99 & 1179.615 & -12 \\
\hline GMSMYEjWNKU & 85 & 451.8662 & 3 & 45.38 & 1352.561 & 11.5 \\
\hline cTLKMSiFFKU & 85 & 357.9353 & 4 & 47.42 & 1427.722 & -6.8 \\
\hline WGKTFVNRCKU & 85 & 413.2216 & 3 & 50.04 & 1236.655 & -9.9 \\
\hline WNKMPTFVEKU & 85 & 426.8854 & 3 & 51.23 & 1277.659 & -19.3 \\
\hline PMYFEjjLYKU & 85 & 503.236 & 3 & 57.26 & 1506.691 & -2.9 \\
\hline YLYFUQHTMKU & 85 & 470.5575 & 3 & 58.41 & 1408.671 & -14.4 \\
\hline LUSSCMVFEKU & 85 & 408.1939 & 3 & 67.99 & 1221.564 & -3 \\
\hline QVFPEQDNNKU & 85 & 609.292 & 2 & 76.71 & 1216.584 & -11.8 \\
\hline XAQNBLEQSKU & 84 & 471.2177 & 3 & 23.45 & 1410.655 & -17.1 \\
\hline EYTZNFbIGKU & 84 & 521.2231 & 3 & 25.05 & 1560.667 & -12.2 \\
\hline GVNXFVbZiKU & 84 & 510.5723 & 3 & 25.16 & 1528.701 & -3.7 \\
\hline AHWXSJNAJKU & 84 & 522.2226 & 3 & 25.4 & 1563.674 & -17.8 \\
\hline LWDJDDRKcKU & 84 & 512.2471 & 3 & 27.31 & 1533.736 & -10.8 \\
\hline EREQKVFGEKU & 84 & 416.8868 & 3 & 28.96 & 1247.662 & -18.8 \\
\hline RAPDAPGJNKU & 84 & 595.7949 & 2 & 28.98 & 1189.591 & -13.1 \\
\hline OCMQDFDCTKU & 84 & 431.8369 & 3 & 29.24 & 1292.485 & 3 \\
\hline QOFYCGLKTKU & 84 & 430.8835 & 3 & 29.51 & 1289.645 & -12.6 \\
\hline ATMUELEjbKU & 84 & 476.5513 & 3 & 29.8 & 1426.645 & -9 \\
\hline KGjPARLjGKU & 84 & 311.6777 & 4 & 30.15 & 1242.689 & -5.7 \\
\hline LFSGIPSKAKU & 84 & 408.8873 & 3 & 30.47 & 1223.651 & -9.2 \\
\hline KYOPECLGVKU & 84 & 463.2451 & 3 & 30.68 & 1386.722 & -6.4 \\
\hline LZTSjTCOUKU & 84 & 480.5419 & 3 & 30.69 & 1438.624 & -14.1 \\
\hline KYLYCjQMYKU & 84 & 483.2292 & 3 & 31.3 & 1446.676 & -6.8 \\
\hline SDCPVTQCQKU & 84 & 468.5563 & 3 & 34.27 & 1402.661 & -9.7 \\
\hline SGGAMSQJYKU & 84 & 398.5119 & 3 & 34.49 & 1192.525 & -9.4 \\
\hline ZSCSPQYRWKU & 84 & 450.2049 & 3 & 35.16 & 1347.614 & -15.9 \\
\hline GVOIDNYLVKU & 84 & 467.8941 & 3 & 35.38 & 1400.68 & -14 \\
\hline DANPYEiHZKU & 84 & 467.8685 & 3 & 35.89 & 1400.598 & -10 \\
\hline XGGPDPGEAKU & 84 & 523.7338 & 2 & 36.09 & 1045.469 & -15.1 \\
\hline YiKSVVLTCKU & 84 & 425.2308 & 3 & 36.21 & 1272.677 & -4.8 \\
\hline TFTEQYKUQKU & 84 & 676.3336 & 2 & 37.26 & 1350.668 & -11.4 \\
\hline GVCQNLGLKKU & 84 & 353.539 & 3 & 37.44 & 1057.607 & -10.9 \\
\hline QLVEMHPCMKU & 84 & 405.5264 & 3 & 37.62 & 1213.577 & -16 \\
\hline
\end{tabular}




\begin{tabular}{|c|c|c|c|c|c|c|}
\hline XAOLLLGVAKU & 84 & 403.2306 & 3 & 38.38 & 1206.685 & -12.2 \\
\hline VCNLMTNDAKU & 84 & 419.2039 & 3 & 44.46 & 1254.609 & -15.2 \\
\hline LQFQbGjSHKU & 84 & 457.5556 & 3 & 47.93 & 1369.668 & -16.7 \\
\hline VTNTFWPCTKU & 84 & 399.1954 & 3 & 48.05 & 1194.586 & -17.9 \\
\hline TYLVMLCNNKU & 84 & 599.3011 & 2 & 49.27 & 1196.605 & -14.1 \\
\hline LVMXVLQOFKU & 84 & 350.9372 & 4 & 53.46 & 1399.741 & -15.1 \\
\hline cYUNQAMLAKU & 84 & 437.8769 & 3 & 55.77 & 1310.627 & -13.8 \\
\hline YDLPMDGPLKU & 84 & 574.2868 & 2 & 59.26 & 1146.574 & -13.2 \\
\hline TVYVUPPDYKU & 84 & 420.8782 & 3 & 60.64 & 1259.63 & -13.6 \\
\hline DMOPAQXLLKU & 84 & 446.8837 & 3 & 61.4 & 1337.652 & -17.3 \\
\hline TjGFGYQNVKU & 84 & 611.2977 & 2 & 61.41 & 1220.591 & -8.2 \\
\hline CTWLNVPLjKU & 84 & 427.8901 & 3 & 72.24 & 1280.667 & -14.4 \\
\hline TCGDMEXHBKU & 83 & 472.1874 & 3 & 23.19 & 1413.547 & -4.4 \\
\hline VGQGEXEPEKU & 83 & 397.8483 & 3 & 24.28 & 1190.543 & -16.6 \\
\hline MDVGSCPUVKU & 83 & 372.1703 & 3 & 25.12 & 1113.506 & -14.9 \\
\hline KPDONCDDLKU & 83 & 417.5255 & 3 & 25.3 & 1249.562 & -6 . \\
\hline DAHXOLTLGKU & 83 & 426.5457 & 3 & 27.07 & 1276.628 & -10.4 \\
\hline DBjDLYLVGKU & 83 & 469.2307 & 3 & 29.11 & 1404.681 & -7.7 \\
\hline KCTJPSHSCKU & 83 & 468.55 & 3 & 34.84 & 1402.643 & -10.7 \\
\hline MSBTMPLPQKU & 83 & 436.2147 & 3 & 34.85 & 1305.638 & -11.7 \\
\hline NBMCQLNDYKU & 83 & 468.1986 & 3 & 37.11 & 1401.597 & -16.5 \\
\hline PDLGGANQUKU & 83 & 539.7697 & 2 & 37.49 & 1077.532 & -6.1 \\
\hline NNDjKPMMYKU & 83 & 450.2054 & 3 & 39.4 & 1347.604 & -6.7 \\
\hline FVSADiHTFKU & 83 & 428.8743 & 3 & 39.98 & 1283.617 & -12.1 \\
\hline FMMQADGHFKU & 83 & 605.77 & 2 & 41.96 & 1209.542 & -13.9 \\
\hline CSEMVOZMLKU & 83 & 446.8657 & 3 & 42.23 & 1337.579 & -2.8 \\
\hline LENKQFCMEKU & 83 & 423.5421 & 3 & 43.15 & 1267.605 & -0.6 \\
\hline EYDPEDMJAKU & 83 & 435.8466 & 3 & 46.19 & 1304.531 & -10.1 \\
\hline VZTPFCMTDKU & 83 & 442.872 & 3 & 47 & 1325.616 & -16.1 \\
\hline KYUVMPTUYKU & 83 & 463.5639 & 3 & 48.22 & 1387.682 & -8.7 \\
\hline VGAVCYPLFKU & 83 & 548.301 & 2 & 53.84 & 1094.595 & -6.7 \\
\hline ESMNNNYYLKU & 83 & 637.7894 & 2 & 55.65 & 1273.576 & -9.4 \\
\hline MFAYTQEMMKU & 83 & 639.7762 & 2 & 57.06 & 1277.561 & -18 \\
\hline LVFMPFHjWKU & 83 & 471.5775 & 3 & 57.84 & 1411.72 & -6.2 \\
\hline TjSOXLAGVKU & 83 & 654.3214 & 2 & 58.36 & 1306.636 & - \\
\hline TVYVULDEMKU & 83 & 426.2104 & 3 & 61.08 & 1275.628 & -14.6 \\
\hline jTGFGXLQVKU & 83 & 639.3156 & 2 & 61.49 & 1276.639 & -17.8 \\
\hline MVZMMNLPQKU & 83 & 643.3063 & 2 & 69.06 & 1284.614 & -12.6 \\
\hline TDMNEVMZLKU & 83 & 637.7891 & 2 & 76.23 & 1273.58 & -12.6 \\
\hline VGEMVPMEcKU & 83 & 606.7864 & 2 & 77.41 & 1211.576 & -14.5 \\
\hline WOLRGADALKU & 83 & 411.5598 & 3 & 77.93 & 1231.669 & -9.1 \\
\hline VSTYBSXSVKU & 82 & 455.5468 & 3 & 23.63 & 1363.643 & -18 \\
\hline TQAETYAAIKU & 82 & 405.8662 & 3 & 24.79 & 1214.58 & -2 \\
\hline UjMMTEjHHKU & 82 & 504.2092 & 3 & 25.04 & 1509.63 & -15.8 \\
\hline FiMDCLPQQKU & 82 & 373.425 & 4 & 25.33 & 1489.696 & -16 \\
\hline
\end{tabular}




\begin{tabular}{|c|c|c|c|c|c|c|}
\hline LPPOKTAGGKU & 82 & 357.8711 & 3 & 25.83 & 1070.61 & -17.1 \\
\hline EEDPGALKTKU & 82 & 362.8613 & 3 & 26 & 1085.572 & -8.6 \\
\hline GUQMHDNcVKU & 82 & 434.5292 & 3 & 27.32 & 1300.581 & -11.7 \\
\hline BMCRUSACVKU & 82 & 500.5612 & 3 & 27.85 & 1498.679 & -11.2 \\
\hline FAWQRQVGEKU & 82 & 416.5578 & 3 & 28.64 & 1246.657 & -4.4 \\
\hline MYHREYRPMKU & 82 & 353.1761 & 4 & 29.8 & 1408.686 & -7.2 \\
\hline ATMUHPHjbKU & 82 & 476.5507 & 3 & 29.87 & 1426.646 & -11.1 \\
\hline SGQMSQGjYKU & 82 & 597.2659 & 2 & 34.84 & 1192.527 & -7.9 \\
\hline XGYEQYVGWKU & 82 & 450.2049 & 3 & 35.17 & 1347.611 & -13.4 \\
\hline VVTNNXBIMKU & 82 & 398.4318 & 4 & 35.66 & 1589.718 & -12.3 \\
\hline KKYPFRYEOKU & 82 & 487.9312 & 3 & 36.88 & 1460.779 & -4.8 \\
\hline VZBYOVLYKKU & 82 & 397.2059 & 4 & 39.83 & 1584.812 & -10.8 \\
\hline DNLHSMHETKU & 82 & 404.1899 & 3 & 41.47 & 1209.556 & -6.8 \\
\hline PGTPCYAPWKU & 82 & 403.8728 & 3 & 42.1 & 1208.606 & -7.5 \\
\hline YSGWYDTFKKU & 82 & 431.8745 & 3 & 43.7 & 1292.619 & -13.4 \\
\hline YPVVQXGTQKU & 82 & 619.8127 & 2 & 47.17 & 1237.632 & -16.7 \\
\hline THLPLTADJKU & 82 & 420.886 & 3 & 52.33 & 1259.658 & -17.2 \\
\hline VDGLTTEFFKU & 82 & 385.8696 & 3 & 55.43 & 1154.597 & -8.8 \\
\hline YLYVFUSUMKU & 82 & 705.3345 & 2 & 58.48 & 1408.671 & -11.7 \\
\hline VVSPYUFGVKU & 82 & 587.8175 & 2 & 59.94 & 1173.629 & -7.6 \\
\hline ZQFSDMMZDKU & 82 & 695.7707 & 2 & 60.36 & 1389.544 & -12.5 \\
\hline KFAWGTCFFKU & 82 & 411.8746 & 3 & 61.46 & 1232.617 & -11.7 \\
\hline VUjYDNYLVKU & 82 & 701.341 & 2 & 62.31 & 1400.681 & -9.4 \\
\hline DSBTVELPQKU & 82 & 430.8828 & 3 & 62.78 & 1289.642 & -11.8 \\
\hline SVGEYXFRiKU & 82 & 480.2266 & 3 & 63.18 & 1437.677 & -13.1 \\
\hline KFUSQbSFFKU & 82 & 472.5672 & 3 & 64.09 & 1414.696 & -11.6 \\
\hline QQMCPNVFEKU & 82 & 408.1933 & 3 & 67.73 & 1221.564 & -4.4 \\
\hline TLQAGAjTKKU & 81 & 375.8776 & 3 & 25.68 & 1124.627 & -14.4 \\
\hline TFFNWGNACKU & 81 & 396.1842 & 3 & 26.68 & 1185.539 & -6.9 \\
\hline PBGYRJTGAKU & 81 & 348.1712 & 4 & 26.73 & 1388.671 & -10.8 \\
\hline QTGVbFNQbKU & 81 & 339.92 & 4 & 27.93 & 1355.662 & -8.2 \\
\hline QLVGACCPSKU & 81 & 502.7474 & 2 & 28.04 & 1003.494 & -14.1 \\
\hline VYNFQTMEJKU & 81 & 356.9165 & 4 & 28.45 & 1423.651 & -10.1 \\
\hline EYVWKVSAGKU & 81 & 583.3152 & 2 & 29.26 & 1164.629 & -11.4 \\
\hline cNYNQETYGKU & 81 & 655.2895 & 2 & 31.25 & 1308.582 & -13.1 \\
\hline DiSGGDQEGKU & 81 & 563.2351 & 2 & 34.34 & 1124.46 & -3.8 \\
\hline ETCDPYPMDKU & 81 & 399.8294 & 3 & 34.79 & 1196.484 & -14.9 \\
\hline TFTEGiKjVKU & 81 & 451.2245 & 3 & 36.95 & 1350.677 & -18.7 \\
\hline LQMGABjSHKU & 81 & 452.2133 & 3 & 40.81 & 1353.638 & -15.1 \\
\hline FMMQADFGHKU & 81 & 605.7715 & 2 & 42.14 & 1209.542 & -11.4 \\
\hline VFAPLPMKTKU & 81 & 377.5577 & 3 & 43.93 & 1129.668 & -15.1 \\
\hline VGODSKGEVKU & 81 & 374.5293 & 3 & 47.11 & 1120.574 & -6.8 \\
\hline TGDFCVFSDKU & 81 & 422.5262 & 3 & 48.08 & 1264.579 & -17.6 \\
\hline KYOPECLVGKU & 81 & 463.2446 & 3 & 49.78 & 1386.722 & -7.4 \\
\hline DYLSYTMCDKU & 81 & 462.5402 & 3 & 51.05 & 1384.604 & -3.4 \\
\hline
\end{tabular}




\begin{tabular}{|c|c|c|c|c|c|c|}
\hline QDNJMELPCKU & 81 & 478.5551 & 3 & 52.12 & 1432.644 & -0.4 \\
\hline QCSTTSFMEKU & 81 & 580.7482 & 2 & 54.21 & 1159.5 & -15.8 \\
\hline LVFEMRQOFKU & 81 & 350.9362 & 4 & 55.15 & 1399.73 & -10 \\
\hline QVFPQEDNNKU & 81 & 609.2924 & 2 & 58.09 & 1216.584 & -11.2 \\
\hline FLSYFUNHHKU & 81 & 457.8909 & 3 & 60.73 & 1370.663 & -8.9 \\
\hline GLLQDTXjKU & 80 & 490.2335 & 3 & 22.63 & 1467.689 & -7.1 \\
\hline IQNMKNQQSKU & 80 & 465.8866 & 3 & 23.42 & 1394.658 & -14.2 \\
\hline LXFGVEGWIKU & 80 & 482.5639 & 3 & 23.98 & 1444.685 & -10.5 \\
\hline WAEWGKVYEKU & 80 & 432.2221 & 3 & 24.09 & 1293.651 & -4.7 \\
\hline cjEDYPbGHKU & 80 & 489.2104 & 3 & 28.65 & 1464.629 & -13.3 \\
\hline cUTAKXWEGKU & 80 & 471.5538 & 3 & 29.14 & 1411.657 & -12.4 \\
\hline TKGHLCFSFKU & 80 & 583.8024 & 2 & 29.51 & 1165.607 & -14.1 \\
\hline HSQOFETCSKU & 80 & 423.8583 & 3 & 30.11 & 1268.547 & 5 \\
\hline FIGLECLVGKU & 80 & 351.6836 & 4 & 30.82 & 1402.716 & -7.6 \\
\hline MYVSMEUPRKU & 80 & 330.6619 & 4 & 30.85 & 1318.627 & -6.9 \\
\hline GPAELAWYFKU & 80 & 394.2086 & 3 & 31.77 & 1179.608 & -3.2 \\
\hline OEFEVSYCUKU & 80 & 463.1979 & 3 & 32.12 & 1386.589 & -12 \\
\hline NDSTDNCTRKU & 80 & 434.2015 & 3 & 32.48 & 1299.587 & -3.3 \\
\hline VQGGGGUGFKU & 80 & 493.2484 & 2 & 33.23 & 984.489 & -6.8 \\
\hline TYGWTECMEKU & 80 & 465.5366 & 3 & 34.87 & 1393.604 & -11.4 \\
\hline YALWKYEETKU & 80 & 443.8924 & 3 & 35.32 & 1328.677 & -16 \\
\hline DANUEVSJUKU & 80 & 463.1985 & 3 & 35.49 & 1386.598 & -17.5 \\
\hline KECFHCMSTKU & 80 & 435.2013 & 3 & 36.52 & 1302.593 & -8.2 \\
\hline DANLDJYSZKU & 80 & 462.5369 & 3 & 39.33 & 1384.596 & -5.4 \\
\hline LCNVjDGSVKU & 80 & 430.8811 & 3 & 40.51 & 1289.64 & -14.3 \\
\hline FMARUDQCQKU & 80 & 466.2195 & 3 & 42.83 & 1395.655 & -12.7 \\
\hline FSCbCLTFKKU & 80 & 324.1584 & 4 & 43.59 & 1292.623 & -14 \\
\hline EQMFjMGZTKU & 80 & 687.7896 & 2 & 44.66 & 1373.583 & -13.3 \\
\hline OACLMBLPQKU & 80 & 461.2234 & 3 & 44.97 & 1380.671 & -16.2 \\
\hline EVAVYMBGXKU & 80 & 464.2122 & 3 & 45.4 & 1389.641 & -19.1 \\
\hline EFGGAGWFEKU & 80 & 563.7639 & 2 & 45.55 & 1125.524 & -10 \\
\hline UAFYADLSMKU & 80 & 408.8594 & 3 & 45.67 & 1223.576 & -15.7 \\
\hline LMVVQXGTQKU & 80 & 611.8155 & 2 & 47.1 & 1221.64 & -19.2 \\
\hline QAVGDVGIWKU & 80 & 417.2061 & 3 & 47.74 & 1248.61 & -11 \\
\hline MCENEVMZLKU & 80 & 645.7866 & 2 & 55.59 & 1289.557 & 1.5 \\
\hline KMSQAMMPMKU & 80 & 394.5243 & 3 & 55.94 & 1180.559 & -6.5 \\
\hline ZQFDMCYYDKU & 80 & 703.7675 & 2 & 56.03 & 1405.543 & -16.2 \\
\hline VGAVGjPLbKU & 80 & 583.8209 & 2 & 56.51 & 1165.639 & -10.4 \\
\hline TECDPYNNDKU & 80 & 599.2408 & 2 & 57.91 & 1196.477 & -8.1 \\
\hline TGDYWBLPQKU & 80 & 461.2246 & 3 & 58.09 & 1380.663 & -7.8 \\
\hline AIDTVELPQKU & 80 & 430.8828 & 3 & 62.58 & 1289.647 & -15.5 \\
\hline LcEDVFFbUKU & 80 & 496.9013 & 3 & 63.21 & 1487.709 & -18.2 \\
\hline ZEYLCCFBFKU & 80 & 507.8778 & 3 & 63.8 & 1520.638 & -17.5 \\
\hline FPVVQNUPDKU & 80 & 611.8168 & 2 & 64.44 & 1221.626 & -5.2 \\
\hline KLTQMNUDSKU & 80 & 622.306 & 2 & 66.18 & 1242.614 & -13.3 \\
\hline
\end{tabular}




\begin{tabular}{|c|c|c|c|c|c|c|}
\hline DjLPLMPGHKU & 80 & 608.3136 & 2 & 69 & 1214.62 & -6.2 \\
\hline KFNCITFFFKU & 80 & 521.5892 & 3 & 76.56 & 1561.765 & -12.2 \\
\hline KPCCYFNFLKU & 80 & 631.3063 & 2 & 77.89 & 1260.615 & -13.2 \\
\hline MGCYTALLFKU & 80 & 382.5272 & 3 & 78.33 & 1144.577 & -15.4 \\
\hline MGTCYALLFKU & 80 & 573.2881 & 2 & 78.34 & 1144.577 & -13.7 \\
\hline PISQMNVFEKU & 80 & 457.2144 & 3 & 80.22 & 1368.635 & -9.7 \\
\hline LEDTZTUDNKU & 79 & 437.1952 & 3 & 22.23 & 1308.569 & -4.2 \\
\hline DPUTSMSCLKU & 79 & 436.8673 & 3 & 23.1 & 1307.599 & -14.8 \\
\hline FDVQQRRLEKU & 79 & 330.1889 & 4 & 23.62 & 1316.731 & -3.6 \\
\hline VQRTFZMESKU & 79 & 440.5538 & 3 & 24.4 & 1318.645 & -4.3 \\
\hline PVNTLbLTHKU & 79 & 310.678 & 4 & 26.18 & 1238.695 & -9.9 \\
\hline jQTVGMjDNKU & 79 & 655.2914 & 2 & 26.32 & 1308.582 & -10.7 \\
\hline PBQTFLMUAKU & 79 & 348.1712 & 4 & 26.86 & 1388.682 & -19.3 \\
\hline DAHXbSLLGKU & 79 & 320.16 & 4 & 27.17 & 1276.624 & -10.3 \\
\hline TYNBTNUMTKU & 79 & 476.2071 & 3 & 28.28 & 1425.626 & -18.6 \\
\hline AGITEECMEKU & 79 & 479.2046 & 3 & 33.39 & 1434.6 & -5.9 \\
\hline KEGHFGUGMKU & 79 & 390.5236 & 3 & 36.57 & 1168.556 & -6 \\
\hline SLSMTCPZTKU & 79 & 581.2649 & 2 & 36.6 & 1160.532 & -14.2 \\
\hline LFSNHGSKAKU & 79 & 363.2021 & 3 & 37.96 & 1086.594 & -8.2 \\
\hline GLHFVQDZSKU & 79 & 408.8685 & 3 & 38.75 & 1223.605 & -17.2 \\
\hline WGFDEYAQEKU & 79 & 424.5221 & 3 & 38.85 & 1270.562 & -13.7 \\
\hline EPPFTGOWSKU & 79 & 417.8665 & 3 & 39.36 & 1250.595 & -13.5 \\
\hline LQMQQFDONKU & 79 & 452.2137 & 3 & 40.89 & 1353.636 & -12.2 \\
\hline LKUCCCCLLKU & 79 & 451.8893 & 3 & 41.19 & 1352.658 & -8.7 \\
\hline DOMDOPNDLKU & 79 & 452.1966 & 3 & 42.07 & 1353.575 & -4.9 \\
\hline YQVSGDANYKU & 79 & 572.2668 & 2 & 43.35 & 1142.536 & -14.4 \\
\hline QQNQYMYKGKU & 79 & 429.5421 & 3 & 43.85 & 1285.624 & -15 \\
\hline DWScVPGMVKU & 79 & 404.5287 & 3 & 44.35 & 1210.588 & -19.9 \\
\hline SMLSZMMVNKU & 79 & 412.1911 & 3 & 44.95 & 1233.567 & -12.5 \\
\hline YTZCGDMTVKU & 79 & 404.5049 & 3 & 46.71 & 1210.511 & -15.1 \\
\hline SESLYMGFGKU & 79 & 559.2628 & 2 & 47.72 & 1116.527 & -14.7 \\
\hline QKYJLTDNNKU & 79 & 463.5639 & 3 & 48.33 & 1387.68 & -7.2 \\
\hline UKRPTAZGEKU & 79 & 420.882 & 3 & 52.2 & 1259.648 & -19 \\
\hline AGCbASFMEKU & 79 & 580.7482 & 2 & 54.21 & 1159.497 & -13.1 \\
\hline GMFLDCPTSKU & 79 & 415.8699 & 3 & 54.92 & 1244.593 & -3.7 \\
\hline VDGVPTFZVKU & 79 & 385.8697 & 3 & 55.3 & 1154.608 & -18.3 \\
\hline QNLETFMjSKU & 79 & 435.873 & 3 & 57.67 & 1304.616 & -14.1 \\
\hline XGGXEXYMFKU & 79 & 497.5334 & 3 & 57.94 & 1489.592 & -9 \\
\hline YLYCFGHTMKU & 79 & 470.558 & 3 & 58.33 & 1408.666 & -10.1 \\
\hline VVAEMHYRGKU & 79 & 594.8112 & 2 & 61.47 & 1187.623 & -13 \\
\hline LNKIDPFDMKU & 79 & 447.5488 & 3 & 64.46 & 1339.646 & -15.9 \\
\hline YLIFUGHTMKU & 79 & 470.5582 & 3 & 78.32 & 1408.658 & -3.5 \\
\hline MZLAFVDXTKU & 79 & 669.8237 & 2 & 91.88 & 1337.63 & 2.3 \\
\hline QQUXDAQGSKU & 78 & 420.8516 & 3 & 23.42 & 1259.55 & -13.8 \\
\hline VYRAFYCCSKU & 78 & 347.4202 & 4 & 25.34 & 1385.662 & -7.2 \\
\hline
\end{tabular}




\begin{tabular}{|c|c|c|c|c|c|c|}
\hline KGAWUPBTQKU & 78 & 343.172 & 4 & 25.56 & 1368.685 & -19.3 \\
\hline RSVNIJFFKKU & 78 & 381.9513 & 4 & 25.81 & 1523.782 & -4 \\
\hline YKjNZjjDFKU & 78 & 409.6808 & 4 & 26.63 & 1634.714 & -12.2 \\
\hline TjSYGCCHFKU & 78 & 627.255 & 2 & 27.4 & 1252.509 & -10.6 \\
\hline YSGDYWTFKKU & 78 & 431.8748 & 3 & 27.54 & 1292.619 & -12.5 \\
\hline SLAMTACTPKU & 78 & 511.2563 & 2 & 30.13 & 1020.51 & -11.4 \\
\hline TAMMLOPKKKU & 78 & 417.5679 & 3 & 30.32 & 1249.69 & -6.4 \\
\hline SPAFZGFZKKU & 78 & 424.2121 & 3 & 30.58 & 1269.626 & -8.6 \\
\hline EODPGNXbDKU & 78 & 472.5247 & 3 & 34.4 & 1414.569 & -11.6 \\
\hline UQJZDMVACKU & 78 & 528.2291 & 3 & 34.44 & 1581.676 & -6.9 \\
\hline CNSAWVVCVKU & 78 & 419.2095 & 3 & 35.53 & 1254.625 & -14.2 \\
\hline ZMGKAFAHNKU & 78 & 599.2942 & 2 & 36.52 & 1196.587 & -11.3 \\
\hline YFSHWGKKCKU & 78 & 321.4155 & 4 & 36.86 & 1281.644 & -8.8 \\
\hline VABVXFLYKKU & 78 & 487.9301 & 3 & 36.99 & 1460.784 & -10.7 \\
\hline UAHFiDVTMKU & 78 & 341.159 & 4 & 37.08 & 1360.621 & -10.5 \\
\hline HFLGGAGBNKU & 78 & 587.7921 & 2 & 37.7 & 1173.585 & -12.6 \\
\hline AFQHjLHCDKU & 78 & 436.2046 & 3 & 38.33 & 1305.601 & -6.8 \\
\hline CFTEQYKUEKU & 78 & 452.2146 & 3 & 38.66 & 1353.614 & 6.3 \\
\hline FHGViVFGYKU & 78 & 429.5506 & 3 & 38.72 & 1285.648 & -13.7 \\
\hline DSEASKEZSKU & 78 & 587.7628 & 2 & 39.12 & 1173.526 & -12.8 \\
\hline UOGLYNDXOKU & 78 & 379.9156 & 4 & 39.22 & 1515.648 & -9.2 \\
\hline NTICKPMMYKU & 78 & 450.2057 & 3 & 39.33 & 1347.6 & -3.9 \\
\hline FVSASjiGFKU & 78 & 428.8743 & 3 & 40.63 & 1283.614 & -9.6 \\
\hline KINDADPGCKU & 78 & 347.162 & 4 & 40.77 & 1384.629 & -7.2 \\
\hline LMPGiDQLSKU & 78 & 407.8724 & 3 & 41.88 & 1220.609 & -11.1 \\
\hline FMMQTCHSVKU & 78 & 605.7711 & 2 & 42.05 & 1209.546 & -14.9 \\
\hline LNKMiPMDMKU & 78 & 335.9122 & 4 & 44.34 & 1339.632 & -9.1 \\
\hline VGcNKCNHYKU & 78 & 419.2037 & 3 & 44.37 & 1254.601 & -9.2 \\
\hline TGYWDBLPQKU & 78 & 461.2234 & 3 & 45.09 & 1380.663 & -10.4 \\
\hline WVYQAENUGKU & 78 & 637.3005 & 2 & 46.93 & 1272.6 & -10.6 \\
\hline QPLTPbiFFKU & 78 & 357.9355 & 4 & 47.37 & 1427.728 & -10.8 \\
\hline KFMFSXKFFKU & 78 & 357.9353 & 4 & 47.65 & 1427.728 & -11.2 \\
\hline ZQUCDFMEKU & 78 & 491.872 & 3 & 47.76 & 1472.613 & -13 \\
\hline AGRMATIMEKU & 78 & 409.8604 & 3 & 48.13 & 1226.577 & -14.1 \\
\hline bPGGYLAVHKU & 78 & 386.8725 & 3 & 49.48 & 1157.616 & -17.7 \\
\hline КbCSККјАiKU & 78 & 363.935 & 4 & 49.92 & 1451.736 & -17.4 \\
\hline QTYDPVMMTKU & 78 & 606.7816 & 2 & 51.18 & 1211.568 & -15.8 \\
\hline cNYHGFMYGKU & 78 & 655.2888 & 2 & 51.29 & 1308.579 & -12.1 \\
\hline cPKDCPLObKU & 78 & 472.5694 & 3 & 51.81 & 1414.682 & 2.9 \\
\hline TMTTYPVMXKU & 78 & 645.8057 & 2 & 54.27 & 1289.601 & -3.1 \\
\hline GJFSDMMZDKU & 78 & 695.7704 & 2 & 55.89 & 1389.54 & -9.8 \\
\hline NEBGDDYADKU & 78 & 434.1786 & 3 & 56.52 & 1299.517 & -2.1 \\
\hline ADUERCFDNKU & 78 & 638.7727 & 2 & 57.31 & 1275.542 & -8.3 \\
\hline bGCLMBLPQKU & 78 & 461.2233 & 3 & 57.96 & 1380.666 & -13.2 \\
\hline QKLYCCHSLKU & 78 & 656.845 & 2 & 60.51 & 1311.684 & -6.3 \\
\hline
\end{tabular}




\begin{tabular}{|c|c|c|c|c|c|c|}
\hline AEYMTbOAFKU & 78 & 461.2068 & 3 & 61.18 & 1380.621 & -16.4 \\
\hline ZEYMTbOAFKU & 78 & 753.3185 & 2 & 63.72 & 1504.648 & -17.2 \\
\hline QMNVDEGPLKU & 78 & 565.2817 & 2 & 64.32 & 1128.56 & -9.8 \\
\hline YALEGKDJBKU & 78 & 488.5708 & 3 & 71.29 & 1462.696 & -3.9 \\
\hline XADDFVLLIKU & 78 & 477.5685 & 3 & 72.55 & 1429.695 & -8.1 \\
\hline DSVFLYMZTKU & 78 & 649.3076 & 2 & 76.64 & 1296.617 & -12.7 \\
\hline VVAEFjbFGKU & 78 & 661.8293 & 2 & 94.3 & 1321.66 & -12.3 \\
\hline KBFDVLTRIKU & 77 & 524.6091 & 3 & 23.17 & 1570.827 & -14 \\
\hline GPYECAXHYKU & 77 & 459.8688 & 3 & 25.18 & 1376.609 & -17.6 \\
\hline KSTACSETSKU & 77 & 377.856 & 3 & 26.06 & 1130.565 & -16.4 \\
\hline XMHNSMFCjKU & 77 & 475.8534 & 3 & 26.88 & 1424.562 & -16.5 \\
\hline LFFNDSDcHKU & 77 & 439.2045 & 3 & 27.34 & 1314.607 & -11.7 \\
\hline NGbcCETNSKU & 77 & 421.8418 & 3 & 29.58 & 1262.525 & -16.8 \\
\hline IAFBLQQFQKU & 77 & 525.5921 & 3 & 29.64 & 1573.77 & -9.6 \\
\hline GOAFRNYCMKU & 77 & 431.5324 & 3 & 30.34 & 1291.582 & -4.8 \\
\hline LFSLMMREAKU & 77 & 306.9167 & 4 & 30.7 & 1223.652 & -11.4 \\
\hline LDVISAGMJKU & 77 & 440.5422 & 3 & 31.38 & 1318.616 & -8.6 \\
\hline DjNGADDEOKU & 77 & 425.8338 & 3 & 33.49 & 1274.5 & -15.7 \\
\hline LAOSMPTUYKU & 77 & 431.8723 & 3 & 35.31 & 1292.62 & -19 \\
\hline YLOYbGjEDKU & 77 & 506.5547 & 3 & 40.44 & 1516.663 & -13.9 \\
\hline QELQYMUAPKU & 77 & 429.5439 & 3 & 43.91 & 1285.624 & -10.7 \\
\hline KMQMSCFQMKU & 77 & 420.8581 & 3 & 44.02 & 1259.565 & -9.6 \\
\hline WLAEOQNPHKU & 77 & 332.1662 & 4 & 44.43 & 1324.654 & -13.5 \\
\hline KCPCYFNFLKU & 77 & 631.3056 & 2 & 45.16 & 1260.615 & -14.4 \\
\hline GVjNMMNFLKU & 77 & 421.2053 & 3 & 45.44 & 1260.608 & -11 \\
\hline KjZSYPYRCKU & 77 & 483.2298 & 3 & 45.99 & 1446.68 & -8.4 \\
\hline PQVOAcYWbKU & 77 & 502.9087 & 3 & 46.16 & 1505.721 & -11.3 \\
\hline AUEFEDTLSKU & 77 & 609.7841 & 2 & 47.45 & 1217.568 & -11.6 \\
\hline AUEGXNTLSKU & 77 & 609.7842 & 2 & 47.78 & 1217.565 & -9 \\
\hline bPGGYLGVHKU & 77 & 382.2012 & 3 & 47.87 & 1143.601 & -16.4 \\
\hline VTNTEAETOKU & 77 & 399.1959 & 3 & 47.96 & 1194.574 & -7.1 \\
\hline LNVLDTMECKU & 77 & 582.7866 & 2 & 48.17 & 1163.568 & -8 \\
\hline GVVDMNCALKU & 77 & 380.5366 & 3 & 49.83 & 1138.588 & -0.5 \\
\hline IPGGFPMVHKU & 77 & 401.5353 & 3 & 52.22 & 1201.593 & -7.7 \\
\hline LGGVFHHGKKU & 77 & 539.8115 & 2 & 53.07 & 1077.62 & -10.3 \\
\hline BVMGVWbVAKU & 77 & 461.2382 & 3 & 53.1 & 1380.7 & -4.9 \\
\hline TKGHLACYFKU & 77 & 583.8041 & 2 & 53.33 & 1165.607 & -11.1 \\
\hline NYcUQAMLAKU & 77 & 437.8779 & 3 & 55.9 & 1310.627 & -11.5 \\
\hline TjEAFSKDEKU & 77 & 421.5318 & 3 & 56.85 & 1261.591 & -13.6 \\
\hline FLSYUPVDSKU & 77 & 617.8056 & 2 & 73.36 & 1233.614 & -14.3 \\
\hline LFSYFHYMSKU & 77 & 441.2142 & 3 & 74.72 & 1320.633 & -9 \\
\hline QVFPQDENNKU & 77 & 609.2919 & 2 & 76.49 & 1216.584 & -12 \\
\hline LAVDVWESQKU & 77 & 587.3084 & 2 & 83.4 & 1172.619 & -14.2 \\
\hline MKKTSOTIFKU & 76 & 366.9371 & 4 & 23 & 1463.731 & -7.9 \\
\hline IXLDRANLVKU & 76 & 480.2522 & 3 & 26.27 & 1437.744 & -6.5 \\
\hline
\end{tabular}




\begin{tabular}{|c|c|c|c|c|c|c|}
\hline EFGGAGSMPKU & 76 & 490.2314 & 2 & 27.06 & 978.4593 & -11.2 \\
\hline YSTUQGQVOKU & 76 & 431.875 & 3 & 27.78 & 1292.612 & -7 \\
\hline QMPKTYDjSKU & 76 & 435.8732 & 3 & 28.19 & 1304.616 & -13.6 \\
\hline DHOGKQMMJKU & 76 & 481.882 & 3 & 28.3 & 1442.637 & -8.5 \\
\hline KjDDLCRVAKU & 76 & 351.6885 & 4 & 28.74 & 1402.735 & -7.3 \\
\hline TKGHFATMFKU & 76 & 583.8024 & 2 & 29.52 & 1165.607 & -14.1 \\
\hline LFSLHcCLAKU & 76 & 408.8873 & 3 & 30.46 & 1223.657 & -13.4 \\
\hline FDGPDDCTRKU & 76 & 434.2005 & 3 & 32.41 & 1299.591 & -8.5 \\
\hline AGCSTTIMEKU & 76 & 580.7499 & 2 & 32.5 & 1159.487 & -1.4 \\
\hline MQQYPRGVMKU & 76 & 309.9088 & 4 & 33 & 1235.627 & -16.8 \\
\hline LNjTZGUSTKU & 76 & 435.2015 & 3 & 36.77 & 1302.604 & -16 \\
\hline BNMRDGNDYKU & 76 & 693.7996 & 2 & 37.02 & 1385.595 & -7.3 \\
\hline DMVTCPNDLKU & 76 & 378.8412 & 3 & 37.11 & 1133.521 & -17.1 \\
\hline FEYODUVHQKU & 76 & 483.5506 & 3 & 37.68 & 1447.649 & -13.4 \\
\hline TWQVFVKARKU & 76 & 421.256 & 3 & 38.87 & 1260.746 & 0.6 \\
\hline LFSLSLCCAKU & 76 & 392.211 & 3 & 40.15 & 1173.63 & -15.8 \\
\hline FEYTCNOZKKU & 76 & 477.5477 & 3 & 40.36 & 1429.631 & -6.8 \\
\hline LYOPCMjEDKU & 76 & 376.1689 & 4 & 40.4 & 1500.662 & -10.2 \\
\hline GCUCNYGPiKU & 76 & 449.1917 & 3 & 41.24 & 1344.562 & -6 \\
\hline GbPQTFMjSKU & 76 & 441.2032 & 3 & 41.66 & 1320.607 & -14.7 \\
\hline SLZFJAMLAKU & 76 & 447.5585 & 3 & 43.35 & 1339.666 & -9.4 \\
\hline FAFCCMTCWKU & 76 & 443.8579 & 3 & 43.72 & 1328.558 & -4.9 \\
\hline MCGMGGGPLKU & 76 & 475.2174 & 2 & 43.73 & 948.4344 & -14.8 \\
\hline LELMbTEEMKU & 76 & 447.5469 & 3 & 44.22 & 1339.633 & -10.8 \\
\hline ACFFYEVGVKU & 76 & 387.8602 & 3 & 44.25 & 1160.569 & -8.7 \\
\hline VGEjKXOZEKU & 76 & 506.2243 & 3 & 47.21 & 1515.68 & -18.8 \\
\hline VEYMSSUSPKU & 76 & 603.7745 & 2 & 47.94 & 1205.55 & -12.7 \\
\hline WQDNWEGFMKU & 76 & 447.195 & 3 & 49.35 & 1338.582 & -13.8 \\
\hline AYPNMTRVHKU & 76 & 405.8789 & 3 & 49.61 & 1214.634 & -16 \\
\hline NEKTFVNCRKU & 76 & 413.221 & 3 & 50.15 & 1236.64 & 1.3 \\
\hline MYPZANjSBKU & 76 & 496.8816 & 3 & 52.02 & 1487.639 & -10.6 \\
\hline QNLGDTCFMKU & 76 & 578.2724 & 2 & 52.39 & 1154.521 & 7.8 \\
\hline WiLGEGcFVKU & 76 & 454.892 & 3 & 52.57 & 1361.671 & -12.6 \\
\hline KAHRTYDjGKU & 76 & 428.5546 & 3 & 53.15 & 1282.65 & -6.3 \\
\hline CTGFDLSTJKU & 76 & 412.8596 & 3 & 55.79 & 1235.556 & 0.7 \\
\hline cLGNQUMQVKU & 76 & 645.8215 & 2 & 58.18 & 1289.638 & -7.4 \\
\hline NiCCEXFFFKU & 76 & 497.534 & 3 & 58.21 & 1489.589 & -5.7 \\
\hline APPPLCVFYKU & 76 & 567.3036 & 2 & 64.14 & 1132.61 & -15.5 \\
\hline LMVVQGXTQKU & 76 & 611.8168 & 2 & 64.39 & 1221.64 & -17 \\
\hline AAFFPTCYLKU & 76 & 580.2931 & 2 & 73.96 & 1158.59 & -15.5 \\
\hline ELLGDTFMCKU & 76 & 578.2717 & 2 & 78.36 & 1154.546 & -15.2 \\
\hline MGFCSTLLFKU & 76 & 573.287 & 2 & 78.44 & 1144.577 & -15.6 \\
\hline KSCYVIVOMKU & 76 & 465.5564 & 3 & 90.87 & 1393.661 & -10.1 \\
\hline DPTSUMSCLKU & 75 & 436.8673 & 3 & 23.22 & 1307.599 & -14.7 \\
\hline XFbUNCTVGKU & 75 & 511.8987 & 3 & 23.65 & 1532.69 & -10.4 \\
\hline
\end{tabular}




\begin{tabular}{|c|c|c|c|c|c|c|}
\hline FSGSSKSjLKU & 75 & 383.5319 & 3 & 26.76 & 1147.596 & -19 \\
\hline KSTYTjSOAKU & 75 & 649.3109 & 2 & 28.12 & 1296.629 & -17.1 \\
\hline GVEEQKVEOKU & 75 & 416.8868 & 3 & 28.97 & 1247.637 & 1.3 \\
\hline SFSCMQDQSKU & 75 & 580.2381 & 2 & 30.05 & 1158.48 & -15.6 \\
\hline TFTEAGYKWKU & 75 & 410.5434 & 3 & 31.39 & 1228.624 & -12.8 \\
\hline DVAXMSDVNKU & 75 & 399.8517 & 3 & 31.54 & 1196.536 & - \\
\hline GHTLKSMIFKU & 75 & 321.1694 & 4 & 34.35 & 1280.657 & -6.3 \\
\hline EYVECGPKFKU & 75 & 400.1996 & 3 & 34.44 & 1197.585 & -6.8 \\
\hline TRRDMPNMBKU & 75 & 474.8949 & 3 & 36.56 & 1421.682 & -13.6 \\
\hline OANMCQFZYKU & 75 & 468.1991 & 3 & 37.01 & 1401.582 & -4.4 \\
\hline GVVDBCSMTKU & 75 & 405.1868 & 3 & 37.05 & 1212.543 & -3.8 \\
\hline PHGEjYVFYKU & 75 & 337.662 & 4 & 37.31 & 1346.638 & -13.9 \\
\hline PGHEYjVFYKU & 75 & 449.8812 & 3 & 37.55 & 1346.638 & -11.8 \\
\hline YNOPGKLVVKU & 75 & 407.5657 & 3 & 38.19 & 1219.694 & -15.1 \\
\hline EFAZHGHEGKU & 75 & 603.2642 & 2 & 42.04 & 1204.537 & -19.5 \\
\hline EGALDEXMTKU & 75 & 606.7644 & 2 & 42.48 & 1211.535 & -17.3 \\
\hline MMBQYNbMHKU & 75 & 525.5563 & 3 & 43.26 & 1573.661 & -8.8 \\
\hline DQDMALjDXKU & 75 & 455.1975 & 3 & 45.98 & 1362.571 & 0.1 \\
\hline LCFDSXSLSKU & 75 & 609.7846 & 2 & 47.62 & 1217.561 & -5.2 \\
\hline AJPYPVZDGKU & 75 & 436.2031 & 3 & 47.91 & 1305.606 & -14 \\
\hline VYRMMGGNMKU & 75 & 395.8564 & 3 & 50.71 & 1184.562 & -12.2 \\
\hline PNYZbQCYLKU & 75 & 530.245 & 3 & 51.6 & 1587.735 & -13.8 \\
\hline jDMSLPLFIKU & 75 & 483.9047 & 3 & 51.62 & 1448.695 & -1.6 \\
\hline EPPFLHTYSKU & 75 & 406.5425 & 3 & 52.13 & 1216.624 & -15.1 \\
\hline RHTKAYDjGKU & 75 & 428.5549 & 3 & 53.06 & 1282.65 & -5.7 \\
\hline bCLYPVDZGKU & 75 & 653.8029 & 2 & 54.4 & 1305.599 & -6.1 \\
\hline KYFFCCQRTKU & 75 & 490.9105 & 3 & 56.24 & 1469.73 & -13.9 \\
\hline NGSCCCFYWKU & 75 & 434.1772 & 3 & 56.42 & 1299.524 & -11.2 \\
\hline jTEAFSbGPKU & 75 & 421.5308 & 3 & 56.87 & 1261.588 & -13.6 \\
\hline KEGLDTFPMKU & 75 & 388.8732 & 3 & 57.7 & 1163.601 & -2.6 \\
\hline QELQMFCPGKU & 75 & 424.2124 & 3 & 59.3 & 1269.626 & $-\varepsilon$ \\
\hline TVYVGXLMMKU & 75 & 420.8783 & 3 & 60.74 & 1259.627 & -10.9 \\
\hline EGTSPPEEQKU & 75 & 550.7649 & 2 & 60.8 & 1099.515 & 0.5 \\
\hline QEMFjTDMHKU & 75 & 687.7889 & 2 & 63.03 & 1373.583 & -14.1 \\
\hline KLTQQCCDSKU & 75 & 622.3068 & 2 & 66.23 & 1242.611 & -9.3 \\
\hline MVFYETQPQKU & 75 & 635.3097 & 2 & 68.97 & 1268.622 & -13.8 \\
\hline YYLAMVSJDKU & 75 & 452.2151 & 3 & 71.82 & 1353.634 & -8 \\
\hline ACMPXRETVKU & 74 & 336.9172 & 4 & 22.74 & 1343.66 & -14.9 \\
\hline jESBVGVDIKU & 74 & 503.2223 & 3 & 22.86 & 1506.673 & -18.4 \\
\hline EVGPHAPINKU & 74 & 394.5311 & 3 & 24.23 & 1180.585 & -11.8 \\
\hline MKGNOPJEFKU & 74 & 473.8901 & 3 & 24.24 & 1418.658 & -6.9 \\
\hline DSEASSKDjKU & 74 & 587.762 & 2 & 24.33 & 1173.523 & -11.7 \\
\hline MZXQLKTTGKU & 74 & 440.8882 & 3 & 27.73 & 1319.652 & -6.8 \\
\hline MAAGSTVOYKU & 74 & 377.5187 & 3 & 28.27 & 1129.545 & -9.6 \\
\hline EFEVSDVECKU & 74 & 395.1756 & 3 & 31.17 & 1182.523 & -14.9 \\
\hline
\end{tabular}




\begin{tabular}{|c|c|c|c|c|c|c|}
\hline TFTEQcPjVKU & 74 & 451.2234 & 3 & 37.06 & 1350.662 & -9.9 \\
\hline TYLVHcGGNKU & 74 & 394.5378 & 3 & 37.72 & 1180.607 & -13 \\
\hline ZMGKPPTMNKU & 74 & 399.8636 & 3 & 38.54 & 1196.579 & -8.6 \\
\hline FHLGXVFGYKU & 74 & 429.5511 & 3 & 38.86 & 1285.647 & $-11 . \varepsilon$ \\
\hline OGULYNDXOKU & 74 & 506.219 & 3 & 39.27 & 1515.648 & -8.2 \\
\hline EGAVAQFAWKU & 74 & 553.2941 & 2 & 40.68 & 1104.572 & \\
\hline PASKYIPGEKU & 74 & 403.8728 & 3 & 42.09 & 1208.606 & -7.5 \\
\hline QELDEYSSDKU & 74 & 606.7643 & 2 & 42.57 & 1211.531 & -13.5 \\
\hline GiVGAMAXGKU & 74 & 572.2663 & 2 & 43.17 & 1142.527 & -7.7 \\
\hline YQVQDSGGYKU & 74 & 572.2668 & 2 & 43.24 & 1142.536 & -14.4 \\
\hline QNDJMELCPKU & 74 & 478.5556 & 3 & 43.77 & 1432.644 & 0. \\
\hline MKKTjXSTEKU & 74 & 460.8958 & 3 & 46.16 & 1379.67 & -3.3 \\
\hline LMGNQAGFSKU & 74 & 351.1795 & 3 & 46.84 & 1050.528 & -10.9 \\
\hline KcGMPPYAGKU & 74 & 571.2916 & 2 & 46.95 & 1140.583 & -12.5 \\
\hline FPVVQGXTQKU & 74 & 611.8151 & 2 & 47 & 1221.637 & -17.2 \\
\hline AYPGGYRPHKU & 74 & 382.2011 & 3 & 47.75 & 1143.594 & -10.6 \\
\hline YjLAYGPPCKU & 74 & 610.2899 & 2 & 48.69 & 1218.583 & -14.2 \\
\hline DQKTFVNRCKU & 74 & 413.2216 & 3 & 50.04 & 1236.64 & 2.6 \\
\hline CGAGGMWVUKU & 74 & 618.2889 & 2 & 51.44 & 1234.573 & -7.9 \\
\hline EPPFLTYHSKU & 74 & 406.5436 & 3 & 51.85 & 1216.624 & -12.3 \\
\hline iPNEDKTVSKU & 74 & 417.547 & 3 & 52.38 & 1249.617 & 1. \\
\hline LELGDTYMCKU & 74 & 586.2695 & 2 & 52.69 & 1170.541 & -14.3 \\
\hline ASFUSSSNKU & 74 & 603.2892 & 2 & 53.04 & 1204.579 & -12.9 \\
\hline NYCDYFYADKU & 74 & 434.1783 & 3 & 56.35 & 1299.523 & -7.7 \\
\hline MiPDVFFFCKU & 74 & 505.5645 & 3 & 63.68 & 1513.7 & -18.5 \\
\hline FPVVQNDUPKU & 74 & 611.8165 & 2 & 64.54 & 1221.626 & -5.8 \\
\hline DjLPLATSYKU & 74 & 608.3129 & 2 & 67.43 & 1214.627 & -12.5 \\
\hline ODALNVPLjKU & 74 & 641.3326 & 2 & 72.22 & 1280.671 & -15.7 \\
\hline FLiYFFANDKU & 74 & 466.5576 & 3 & 80.84 & 1396.668 & -12.3 \\
\hline KMQIcTOQMKU & 73 & 396.441 & 4 & 22.84 & 1581.722 & 7.9 \\
\hline EVGVAbGbSKU & 73 & 394.5322 & 3 & 24.07 & 1180.587 & -10.5 \\
\hline HWSTTFKLGKU & 73 & 401.8868 & 3 & 24.9 & 1202.656 & -14.5 \\
\hline YUQMDEYFKKU & 73 & 477.5469 & 3 & 24.93 & 1429.645 & -18 \\
\hline VFSGTQICPKU & 73 & 449.8888 & 3 & 25.93 & 1346.655 & -7.8 \\
\hline КНАНСМјАНКU & 73 & 473.5646 & 3 & 26.78 & 1417.682 & -7.1 \\
\hline YNTTSCYiLKU & 73 & 491.8972 & 3 & 29.14 & 1472.687 & -11.5 \\
\hline TVAMTCPATKU & 73 & 511.2563 & 2 & 30.12 & 1020.51 & -11.4 \\
\hline cNYGGUcYGKU & 73 & 663.2864 & 2 & 31.3 & 1324.575 & -12.3 \\
\hline LGPCNBTVGKU & 73 & 388.1959 & 3 & 35.1 & 1161.577 & -9.4 \\
\hline QYEGEWVFYKU & 73 & 337.6625 & 4 & 37.45 & 1346.629 & -6.2 \\
\hline HFLHGANZGKU & 73 & 587.7925 & 2 & 37.79 & 1173.579 & -7.3 \\
\hline DSBTVEPLQKU & 73 & 430.8831 & 3 & 40.86 & 1289.642 & -11.1 \\
\hline YLOYQMGBCKU & 73 & 495.2187 & 3 & 41.37 & 1482.645 & -7.3 \\
\hline DTCMVOMZLKU & 73 & 669.796 & 2 & 42.19 & 1337.579 & -1.2 \\
\hline ZTTLNUEESKU & 73 & 432.5325 & 3 & 42.66 & 1294.59 & -11. \\
\hline
\end{tabular}




\begin{tabular}{|c|c|c|c|c|c|c|}
\hline QELGAYFBGKU & 73 & 429.5435 & 3 & 44.15 & 1285.626 & -13.2 \\
\hline LVDKGTcOVKU & 73 & 419.5649 & 3 & 45.84 & 1255.687 & -10.9 \\
\hline YFEAUPPYSKU & 73 & 427.5348 & 3 & 46.94 & 1279.599 & -12.5 \\
\hline KATjDDVYQKU & 73 & 425.8735 & 3 & 47.02 & 1274.623 & -18.7 \\
\hline TYLVVMQCNKU & 73 & 599.3011 & 2 & 49.27 & 1196.605 & -14.1 \\
\hline GVjNDENFLKU & 73 & 415.2019 & 3 & 52.11 & 1242.596 & -10 \\
\hline WVYLTAZGEKU & 73 & 420.882 & 3 & 52.2 & 1259.63 & -4.4 \\
\hline KMFHQDFFFKU & 73 & 344.1741 & 4 & 54.06 & 1372.675 & -5.6 \\
\hline EcFSDMZMDKU & 73 & 695.7695 & 2 & 56.23 & 1389.541 & -11.9 \\
\hline MbYMYFAGIKU & 73 & 506.8915 & 3 & 58.02 & 1517.65 & 1.5 \\
\hline AGVFPYjDEKU & 73 & 617.2896 & 2 & 58.16 & 1232.58 & -12.2 \\
\hline MGFQFELHFKU & 73 & 428.2125 & 3 & 67.64 & 1281.633 & -13.5 \\
\hline DjYMTAMIFKU & 73 & 502.5476 & 3 & 68.18 & 1504.63 & -6 \\
\hline FLSYPCAESKU & 73 & 617.8055 & 2 & 73.68 & 1233.611 & -11.6 \\
\hline KOAECjKFGKU & 73 & 441.5538 & 3 & 94.61 & 1321.643 & -2.8 \\
\hline GVSVCJPFKKU & 72 & 345.1844 & 4 & 21.05 & 1376.722 & -10.1 \\
\hline KDVKCXYFKKU & 72 & 345.1845 & 4 & 21.06 & 1376.713 & -3.3 \\
\hline EVGQWATTYKU & 72 & 591.3046 & 2 & 23.9 & 1180.588 & 6 \\
\hline ASGGLPECJKU & 72 & 563.7604 & 2 & 24.51 & 1125.519 & -11.6 \\
\hline LTFFHNODLKU & 72 & 335.1707 & 4 & 24.89 & 1336.679 & -18.9 \\
\hline LDcGTIYTLKU & 72 & 335.1711 & 4 & 25.07 & 1336.661 & -4.1 \\
\hline PXQCRGKVEKU & 72 & 421.8831 & 3 & 25.36 & 1262.641 & -11 \\
\hline KHTPVULTHKU & 72 & 310.678 & 4 & 26.18 & 1238.7 & -13.6 \\
\hline LKTbYDGDSKU & 72 & 415.2061 & 3 & 26.67 & 1242.606 & -7.5 \\
\hline QTVGAFFJcKU & 72 & 452.8913 & 3 & 27.93 & 1355.666 & -10.2 \\
\hline KMGYTjSQFKU & 72 & 433.2095 & 3 & 28.04 & 1296.626 & -14.6 \\
\hline SKTYMJAGFKU & 72 & 649.3109 & 2 & 28.12 & 1296.624 & -12.9 \\
\hline OSEBXARbVKU & 72 & 535.917 & 3 & 28.66 & 1604.744 & -9 \\
\hline CHZPDYPBHKU & 72 & 489.2116 & 3 & 28.82 & 1464.616 & -1.9 \\
\hline cGXGGQVMXKU & 72 & 655.2902 & 2 & 31.17 & 1308.572 & -4.7 \\
\hline FOHZAANVHKU & 72 & 331.1606 & 4 & 31.86 & 1320.634 & -15.4 \\
\hline KTDJEQGMTKU & 72 & 434.8699 & 3 & 32.96 & 1301.599 & -8.6 \\
\hline FKKAFVEPFKU & 72 & 310.6844 & 4 & 33.57 & 1238.718 & -7.4 \\
\hline WGKNVFEKCKU & 72 & 413.22 & 3 & 33.83 & 1236.644 & -4.6 \\
\hline BQDKERTAIKU & 72 & 385.9431 & 4 & 34.74 & 1539.745 & -1 \\
\hline SPYCPSHEjKU & 72 & 468.55 & 3 & 34.84 & 1402.63 & -1.3 \\
\hline TYKMACIMEKU & 72 & 465.5352 & 3 & 35 & 1393.605 & -15 \\
\hline LEFEGCKOOKU & 72 & 454.2169 & 3 & 35.76 & 1359.637 & -5.5 \\
\hline LFSLAAHMAKU & 72 & 363.2013 & 3 & 38.15 & 1086.601 & -17.4 \\
\hline IQNMQNKQSKU & 72 & 465.8959 & 3 & 39.23 & 1394.658 & 5.8 \\
\hline FNTHbNMOOKU & 72 & 506.2189 & 3 & 39.37 & 1515.662 & -17.9 \\
\hline EGTSPPEQEKU & 72 & 550.7651 & 2 & 39.4 & 1099.515 & 1 \\
\hline YLOYXTYEDKU & 72 & 506.555 & 3 & 40.33 & 1516.659 & -10.8 \\
\hline FVSAjYAGFKU & 72 & 399.8647 & 3 & 40.34 & 1196.595 & -19.1 \\
\hline IACPAiNLLKU & 72 & 339.1686 & 4 & 41.2 & 1352.663 & -12.9 \\
\hline
\end{tabular}




\begin{tabular}{|c|c|c|c|c|c|c|}
\hline MXVGOVHLYKU & 72 & 457.2256 & 3 & 42.25 & 1368.673 & -13.4 \\
\hline SGDVNRMbEKU & 72 & 418.197 & 3 & 45 & 1251.585 & -12.2 \\
\hline EFAZCCTEGKU & 72 & 665.2773 & 2 & 45.59 & 1328.552 & -8.9 \\
\hline LLPCWCQSAKU & 72 & 574.2831 & 2 & 47.3 & 1146.568 & -14.2 \\
\hline DADXVFLYKKU & 72 & 330.1691 & 4 & 47.38 & 1316.662 & -11.5 \\
\hline UNSDDIWYFKU & 72 & 496.5421 & 3 & 47.64 & 1486.613 & -5.8 \\
\hline VTNTFPCTWKU & 72 & 399.1954 & 3 & 48.05 & 1194.586 & -17.9 \\
\hline DNMAUISCLKU & 72 & 481.5457 & 3 & 49.86 & 1441.634 & -13.1 \\
\hline KMSAINKjFKU & 72 & 484.9108 & 3 & 49.94 & 1451.717 & -4.1 \\
\hline DNbUMSSCLKU & 72 & 481.5463 & 3 & 50 & 1441.629 & -8.2 \\
\hline TiLLTAGEZKU & 72 & 420.8868 & 3 & 52.47 & 1259.638 & 0.7 \\
\hline FDTWMTVEGKU & 72 & 404.8596 & 3 & 54.31 & 1211.565 & -6.3 \\
\hline AAFFPCTYLKU & 72 & 387.1967 & 3 & 55.06 & 1158.59 & -18.4 \\
\hline KYICCNYHYKU & 72 & 504.5459 & 3 & 55.44 & 1510.634 & -11.9 \\
\hline YFEAFSATbKU & 72 & 427.5357 & 3 & 55.63 & 1279.605 & -15.6 \\
\hline KMDGODLGMKU & 72 & 399.856 & 3 & 56.15 & 1196.554 & -6.8 \\
\hline YFEAFMRGDKU & 72 & 631.7956 & 2 & 57.01 & 1261.591 & -11.6 \\
\hline LYGOAPFVHKU & 72 & 412.2167 & 3 & 58.88 & 1233.652 & -19.1 \\
\hline TjOGYLGAVKU & 72 & 610.8073 & 2 & 59.31 & 1219.618 & -14.6 \\
\hline TVYVUFGHMKU & 72 & 420.8783 & 3 & 60.74 & 1259.623 & -8.2 \\
\hline LNCRBPFDMKU & 72 & 447.5488 & 3 & 64.46 & 1339.635 & -7.4 \\
\hline VNFDVMRNVKU & 72 & 407.5525 & 3 & 67.12 & 1219.65 & -11.4 \\
\hline QKMEASFMEKU & 72 & 409.8607 & 3 & 68.2 & 1226.579 & -15.2 \\
\hline MVFYGWMPQKU & 72 & 643.3063 & 2 & 69.06 & 1284.615 & -12.9 \\
\hline AAFCAQQYLKU & 72 & 571.2868 & 2 & 77.62 & 1140.575 & -13.9 \\
\hline AAFQMNGYLKU & 72 & 571.2888 & 2 & 77.76 & 1140.575 & -10.5 \\
\hline VVAEUHWAOKU & 72 & 441.5543 & 3 & 94.51 & 1321.654 & -9.9 \\
\hline ADCPYEQJDKU & 71 & 445.185 & 3 & 24.19 & 1332.536 & -2.3 \\
\hline HPLYNCCHMKU & 71 & 415.5172 & 3 & 24.82 & 1243.541 & -9.2 \\
\hline SSAAPPAQMKU & 71 & 493.7539 & 2 & 25.92 & 985.5015 & -8.3 \\
\hline VGSYGVWISKU & 71 & 405.8722 & 3 & 25.96 & 1214.595 & -0.3 \\
\hline SSAAPPCVQKU & 71 & 493.7527 & 2 & 25.99 & 985.5015 & -10.7 \\
\hline AXKVSAOPGKU & 71 & 394.2042 & 3 & 28.18 & 1179.612 & -18.1 \\
\hline GTBbCZVMTKU & 71 & 476.2077 & 3 & 28.18 & 1425.615 & -9.6 \\
\hline QMLNVYDjSKU & 71 & 435.8732 & 3 & 28.2 & 1304.616 & -13.6 \\
\hline UcTASATWBKU & 71 & 471.5538 & 3 & 29.15 & 1411.651 & -8.3 \\
\hline IFGLEPLFYKU & 71 & 351.6836 & 4 & 30.83 & 1402.714 & -5.9 \\
\hline cNYFGHMYGKU & 71 & 655.2906 & 2 & 31.55 & 1308.579 & -9.3 \\
\hline EPPEGEUVSKU & 71 & 575.774 & 2 & 38.08 & 1149.542 & -6.9 \\
\hline YQTCLLLNLKU & 71 & 403.2306 & 3 & 38.38 & 1206.679 & -7.9 \\
\hline FEYOANUEFKU & 71 & 358.4121 & 4 & 40.31 & 1429.627 & -5.7 \\
\hline SCBPAINLLKU & 71 & 339.1686 & 4 & 41.09 & 1352.658 & -9.2 \\
\hline FAMASSDETKU & 71 & 543.2419 & 2 & 41.35 & 1084.486 & -15.3 \\
\hline AFMAPSCMTKU & 71 & 543.2419 & 2 & 41.35 & 1084.487 & -16.2 \\
\hline EFAACCTEGKU & 71 & 603.2641 & 2 & 43.48 & 1204.525 & -9.1 \\
\hline
\end{tabular}




\begin{tabular}{r|rrrrrr} 
KIECQAYPAKU & 71 & 443.21 & 3 & 43.6 & 1326.624 & -12.2 \\
KYFDLSQZTKU & 71 & 441.89 & 3 & 44.18 & 1322.662 & -10.4 \\
ADSMYEIbGKU & 71 & 451.1892 & 3 & 45.66 & 1350.56 & -10.3 \\
RLWGCTFGVKU & 71 & 419.5649 & 3 & 45.84 & 1255.69 & -14 \\
GNbDDDMTVKU & 71 & 404.5054 & 3 & 46.61 & 1210.511 & -13.4 \\
LLZCGMFSAKU & 71 & 582.28 & 2 & 47.47 & 1162.563 & -14.8 \\
LYMMVGOBSKU & 71 & 469.5515 & 3 & 48.48 & 1405.655 & -15.7 \\
TYLVVNGCWKU & 71 & 591.3021 & 2 & 49.57 & 1180.606 & -14.1 \\
EKLRKLELKU & 71 & 356.2147 & 4 & 49.68 & 1420.847 & -12.1 \\
QTYDPNCKTKU & 71 & 598.7839 & 2 & 51.21 & 1195.566 & -10.3 \\
QGVLCPYVEKU & 71 & 567.7949 & 2 & 51.77 & 1133.59 & -13.3 \\
FVRMANEBFKU & 71 & 472.5692 & 3 & 51.88 & 1414.698 & -8.7 \\
GTFLNSSSKU & 71 & 603.2894 & 2 & 52.93 & 1204.579 & -12.6 \\
USGGQPFjLKU & 71 & 407.8711 & 3 & 53.06 & 1220.602 & -8.8 \\
MMOETECGjKU & 71 & 477.8623 & 3 & 55.14 & 1430.587 & -15.3 \\
KPWQCIEWLKU & 71 & 377.6854 & 4 & 57.73 & 1506.73 & -11.3 \\
VVSFVCPPOKU & 71 & 393.5447 & 3 & 60.1 & 1177.618 & -4.9 \\
VUWWDXGLVKU & 71 & 701.3401 & 2 & 62.42 & 1400.685 & -13.8 \\
CPGLDEXMTKU & 71 & 606.7625 & 2 & 66.8 & 1211.518 & -6 \\
FTFQSMDbGKU & 71 & 639.2849 & 2 & 68.08 & 1276.573 & -13.6 \\
YTLVVMEEAKU & 71 & 591.3036 & 2 & 71.28 & 1180.616 & -19.9 \\
YYLAFGDCQKU & 71 & 452.2148 & 3 & 71.75 & 1353.642 & -14.3 \\
INEPFFYQWKU & 71 & 516.8998 & 3 & 74.12 & 1547.705 & -17.5 \\
AAFAQCQYLUU & 71 & 571.2877 & 2 & 77.87 & 1140.575 & -12.4 \\
YLYVFSIPCKU & 71 & 470.5573 & 3 & 78.51 & 1408.67 & -14.2 \\
LFiYVNEDHKU & 71 & 466.5576 & 3 & 80.85 & 1396.664 & -9.5 \\
MXYVSVKWQKU & 71 & 694.3459 & 2 & 93.63 & 1386.698 & -14.7
\end{tabular}


ELISA

\section{Buffer A (1x PBS, pH = $7.2+0.05 \%$ Tween 20)}

\section{Buffer B (FBS 10\%, 0.05\% Tween 20, 1x PBS, pH = 7.2)}

An ELISA plate (96-well format) was washed with buffer A ( 3 × $200 \mu \mathrm{L}$ per well). Nucleobase peptide library, peptide library or peptide 38 were added, each to three wells (each $100 \mu \mathrm{L}, 100 \mu \mathrm{M}$ in buffer A) and incubated for $1 \mathrm{~h}$ at room temperature. After this time, the supernatant was removed and the wells treated with blocking buffer (buffer $\mathrm{B}, 100 \mu \mathrm{L}$ per well) and incubated overnight. A DNA library (Biotin$\mathrm{N}_{20}, 50 \mu \mathrm{M}$ in buffer $\mathrm{A}$ ) was heated to $95^{\circ} \mathrm{C}$ for $5 \mathrm{~min}$ and let cool down to room temperature over 15 minutes. DNA library ( $50 \mu \mathrm{M}$ in buffer $\mathrm{A}, 100 \mu \mathrm{L}$ per well) was added to all wells and incubated for $1 \mathrm{~h}$ at room temperature. The supernatant was removed, and the wells washed $(2 \times 200 \mu \mathrm{L}$ buffer A per well). SA-HRP (1:2000 dilution) was added to each well and incubated 30 minutes. All wells were washed $(2 \mathrm{x}$ $200 \mu \mathrm{L}$ buffer A per well). TMB ELISA substrate was added to the wells $(50 \mu \mathrm{L})$ and after 5 minutes quenched with sulfuric acid $(50 \mu \mathrm{L})$. The absorbance at $450 \mathrm{~nm}$ was determined for all treated wells.

Affinity selection

Buffer A: Tris $10 \mathrm{mM} \mathrm{pH}=7.5 ; \mathrm{NaCl} 150 \mathrm{mM} ; \mathrm{MgCl}_{2} 10 \mathrm{mM}$

Buffer B: Tris 20mM pH = 7.5; NaCl 300 mM; $\mathrm{MgCl}_{2} 20$ mM; Tween 0.05 \%; Yeast RNA 0.2 mg/mL; Biotin $2 \mathrm{mM}$

Buffer C: Guanidine-HCl 6 M, pH = 6.8; Phosphate 200 mM

Pre-miR-21 or pre-miR-155 $(10 \mu \mathrm{M}, 50 \mu \mathrm{L})$ was diluted to $500 \mu \mathrm{L}$ with buffer $\mathrm{A}$ and heated to $95^{\circ} \mathrm{C}$ for 5 minutes and then left cooling down to room temperature for 15 minutes. $400 \mu \mathrm{L}(4 \mathrm{mg})$ MyOne streptavidin beads were washed with buffer $A(2 \times 1 \mathrm{~mL})$ and then incubated with biotin-pre-miRNA (21 or $155 ; 500 \mu \mathrm{L}, 1 \mu \mathrm{M}$ in Buffer A) at $4^{\circ} \mathrm{C}$ for 30 minutes. The supernatant was removed, and the beads washed with buffer $C(3 \times 1 \mathrm{~mL})$. The beads (for each miRNA) were divided in 4 parts and incubated with buffer $\mathrm{B}(800 \mu \mathrm{L})+$ library $(200 \mu \mathrm{L}, 6.25 \mathrm{mM}$ in $1 \mathrm{X}$ PBS $+5 \% \mathrm{DMF})$ for 120 minutes at $4{ }^{\circ} \mathrm{C}$. After this time the supernatant removed and the beads washed with $2 \times 500 \mu \mathrm{L}$ Buffer $A$. To elute bound library members the beads were treated with $2 \times 100 \mu \mathrm{L}$ Buffer C. All samples were desalted with C18 ZipTips and lyophilized. The samples were taken up in water with $0.1 \%$ formic acid and analyzed by nanoLC-MS/MS. In parallel a control selection was performed on beads not loaded with miRNA, all other steps were identical. 
General procedure for biolayer interferometry (BLI)

Lyophilized peptides biotinylated on C-term lysine was dissolved to $1 \mathrm{mg} / \mathrm{mL}$ in $1 \times$ PBS and diluted 50 -fold into $0.02 \%$ Tween-20, 1x PBS ('kinetic buffer') for immobilization onto streptavidin Octet biosensors (ForteBio; Menlo Park, CA). Biolayer interferometry (BLI) assays were performed in 96 well plates (GreinerBio-One; Kremsmünster, Austria; polypropylene, flat-bottom, chimney well) using an Octet Red96 System (ForteBio; Menlo Park, CA). Wells were filled with $200 \mu \mathrm{L}$ of kinetic buffer, peptide solution, or RNA solution (prepared in kinetic buffer). Biotinylated peptide was immobilized onto the streptavidin tip for $120 \mathrm{~s}$. Sensors were then dipped into kinetic buffer for $60 \mathrm{~s}$, RNA solution $(1 \mu \mathrm{M}, 500 \mathrm{nM}, 100 \mathrm{nM}, 20$ $\mathrm{nM}, 4 \mathrm{nM}$ or $0.8 \mathrm{nM}$ ) for $300 \mathrm{~s}$, and finally into kinetic buffer for $300 \mathrm{~s}$. Measurements were carried out at $30^{\circ} \mathrm{C}$. 

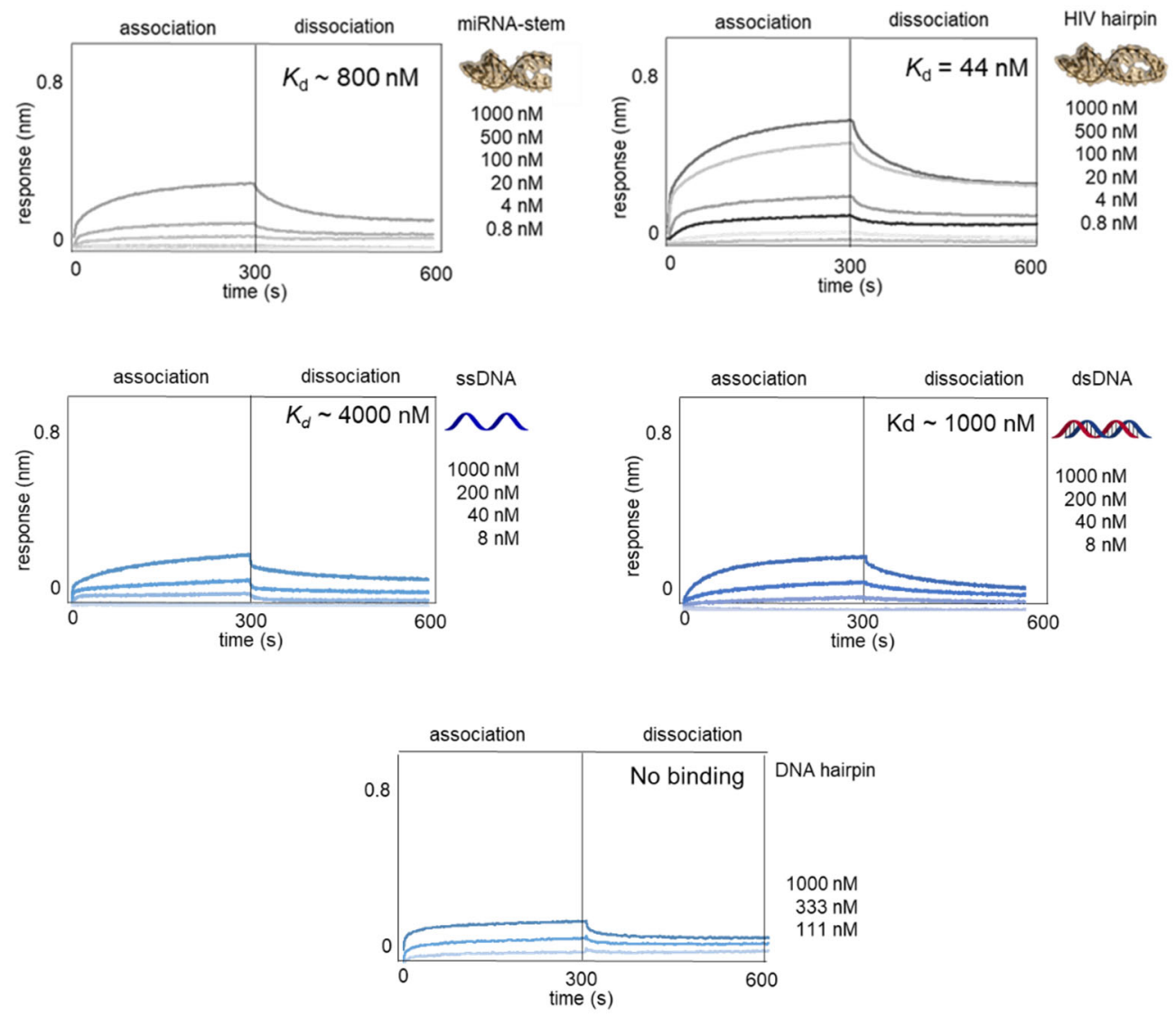

SI Figure 1. BLI curves ds-miRNA-stem, HIV hairpin, ssDNA, dsDNA and DNA hairpin. BLI tips with immobilized nucleobase peptide were dipped into solution containing RNA (association, $300 \mathrm{sec}$ ). RNA concentration range: $1000 \mathrm{nM}, 500 \mathrm{nM}, 100 \mathrm{nM}, 20 \mathrm{nM}, 4 \mathrm{nM}, 0.8 \mathrm{nM}$. Dissociation was performed for $300 \mathrm{sec}$ in kinetic buffer. miRNA-stem sequence: UAGCUUAUCAGACUGAUGUUG; CAACACCAGUCGAUGGGCUG. RNA harpin sequence: CGUGUCUUGGAGUGCUGAUCGGACACC. SSDNA and dsDNA sequence: CCGGCTGACACGTGGTATTAAT. DNA hairpin sequence: AGCTTATCAGACTGATGTTGACTGTTGAATCTCATGGCAACACC AGTCGATGGGCT. All oligonucleotides before usage were heated to $95^{\circ} \mathrm{C}$ for 5 minutes and let cool to room temperature over 15 minutes. 


\section{BLI curves alanine scan}
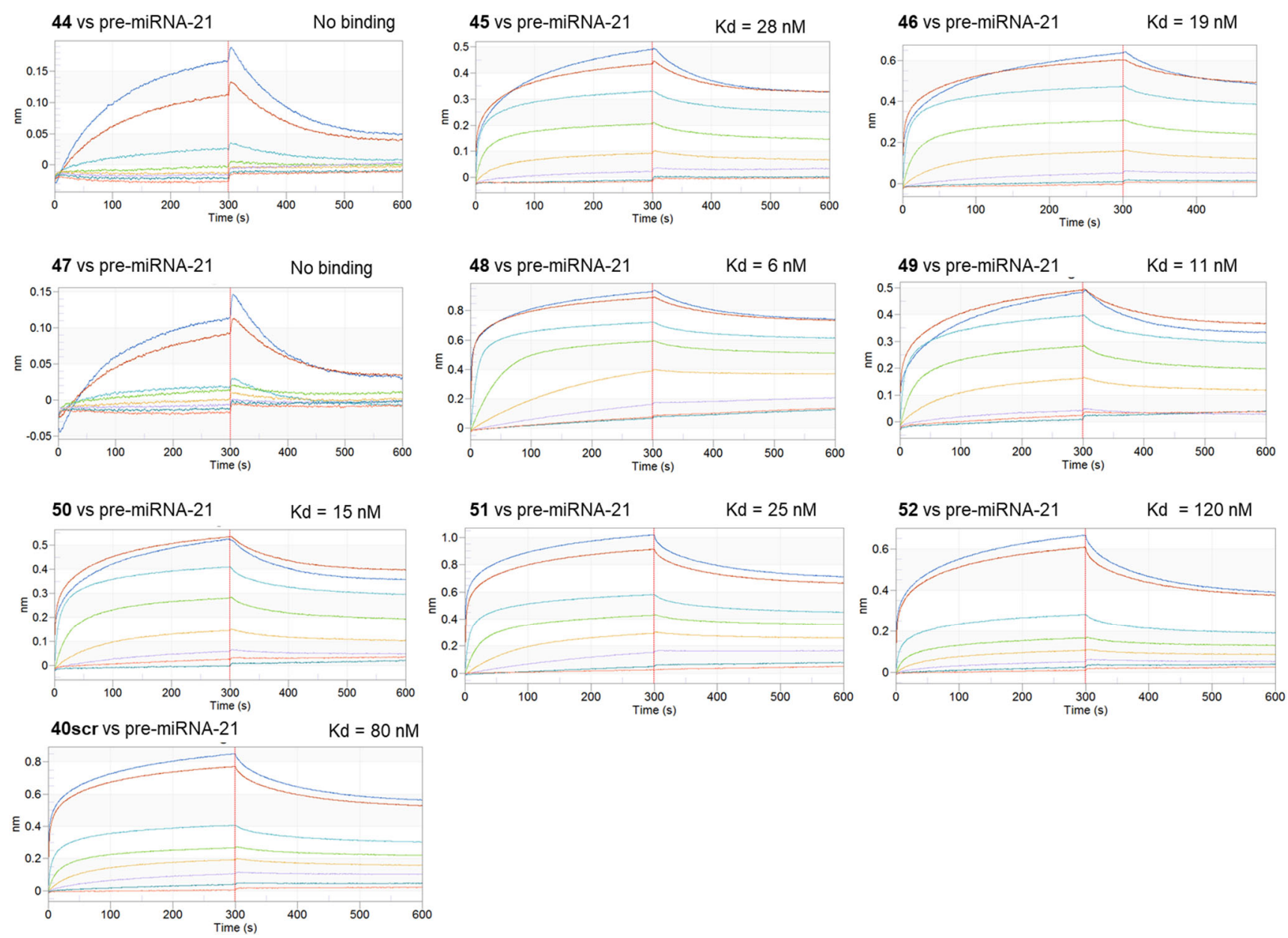

SI Figure 2. Biolayer interferometry association and dissociation curves for alanine scan and scrambled hit. BLI tips with immobilized nucleobase peptide were dipped into solution containing pre-miRNA21 (association, $300 \mathrm{sec}$ ). RNA concentration range: $1000 \mathrm{nM}, 500 \mathrm{nM}, 100 \mathrm{nM}, 20 \mathrm{nM}, 4 \mathrm{nM}, 0.8 \mathrm{nM}$. Dissociation was performed for $300 \mathrm{sec}$ in kinetic buffer. 

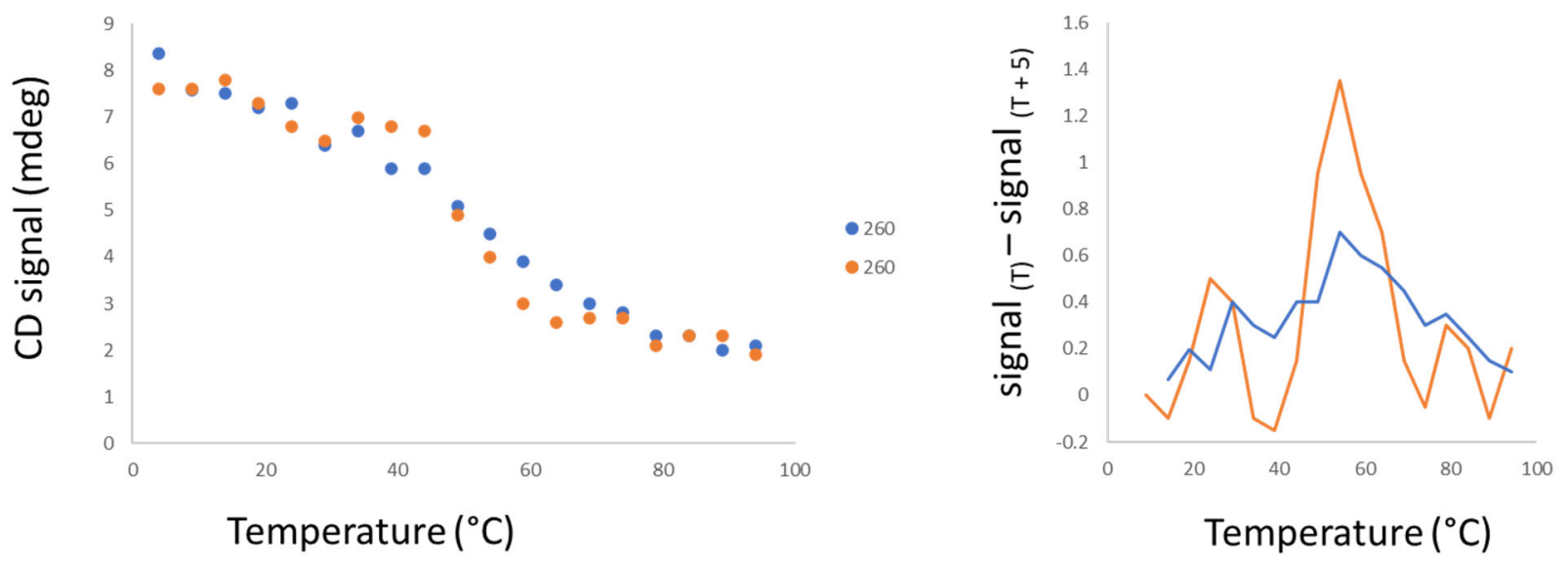

SI Figure 3. Melting curves of pre-miRNA-21 and pre-miRNA-21 + 39, determined by circular dichroism (CD) at $260 \mathrm{~nm}$. Samples were dissolved in $10 \mathrm{mM} \mathrm{MES,} 150 \mathrm{mM} \mathrm{KCl}, 1 \mathrm{mM} \mathrm{MgCl}, 10 \%$ glycerol at $5 \mu \mathrm{M}$ final concentration. Prior to measurement samples were heated to $95^{\circ} \mathrm{C}$ for 5 minutes and cooled down to room temperature over 15 minutes. 